\title{
Examining the macro-evolution and genetic background of complex multicellular structures in mushroom-forming fungi (Agaricomycetes)
}

\author{
Ph.D. Thesis \\ Torda Varga \\ Supervisor: Dr. László G. Nagy
}

Doctoral School of Biology

Synthetic and Systems Biology Unit, Institute of Biochemistry, Biological Research Centre

SZTE TTIK

2020

Szeged 


\section{Contents}

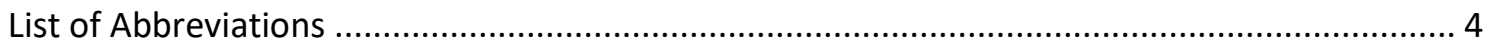

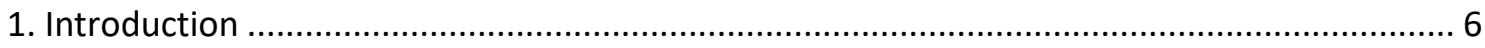

1.1. Multicellularity is a widespread evolutionary innovation.................................................. 6

1.2. Complex multicellularity in fungi ................................................................................. 7

1.2.1. The evolution of complex multicellularity in fungi ................................................... 7

1.2.2. The morphological complexity of mushroom-forming fungi .................................... 9

1.3. Macro-evolution of mushroom-forming fungi............................................................... 13

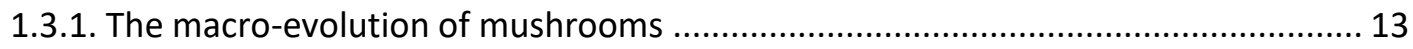

1.3.2. The background of phylogenetic comparative methods used in this study ............. 14

1.4. The genetic background of mushroom development: the model organism Coprinopsis

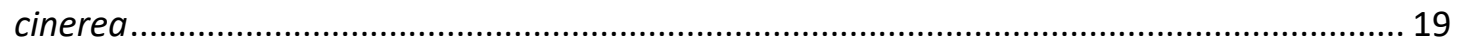

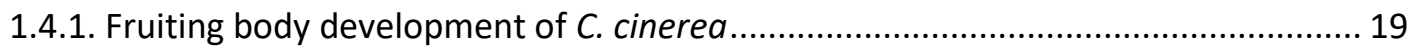

1.4.2. RNA-seq analyses to examine the genetic background of morphological

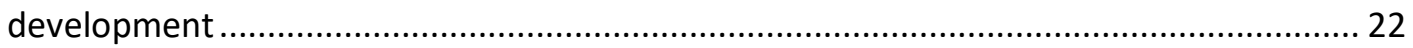

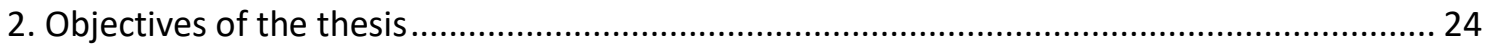

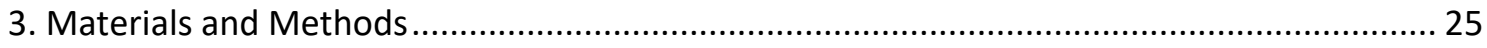

3.1. Comparative phylogenetic methods ............................................................................. 25

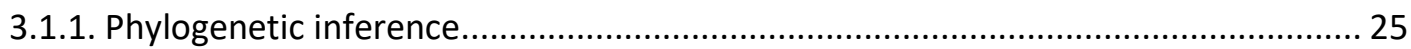

3.1.2. Accounting for non-random and incomplete taxon sampling ................................ 30

3.1.3. Trait independent diversification analyses ............................................................ 30

3.1.4. Macro-evolutionary analyses of morphological traits ............................................. 32

3.2. Laser-capture microdissection coupled low-input RNA sequencing ............................... 38

3.2.1. Exploring tissue differentiation of Coprinopsis cinerea ............................................ 38

3.2.2. Optimizing a tissue-specific low-input RNA-seq workflow ..................................... 38

3.2.3. Quality check and analysis of RNA sequencing data................................................. 43

3.2.4. Defining upregulated genes in hyphal knots and tissue enhanced genes across the

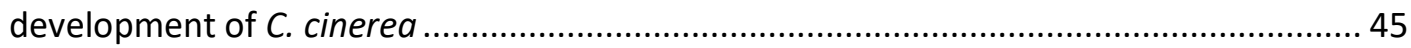

3.2.5. Functional analysis of upregulated and tissue enhanced genes............................. 45

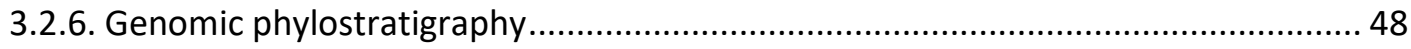

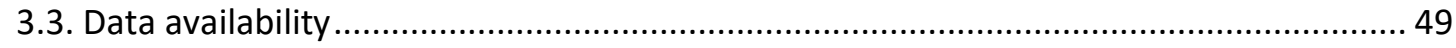

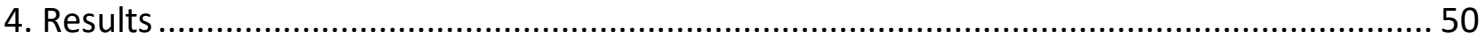

4.1. Comparative phylogeny of mushroom-forming fungi ...................................................... 50

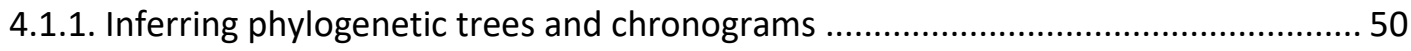

4.1.2. Accounting for non-random and incomplete taxon sampling ................................. 56

4.1.3. Trait independent diversification of mushroom-forming fungi.............................. 57 
4.1.4. Extinction events through the evolution of Agaricomycetes.................................... 58

4.1.5. Main shifts in the diversification rate of mushrooms ............................................ 61

4.1.6. Reconstructing ancestral character states of main fruiting body types ................... 62

4.1.7. Morphological character evolution of mushroom-forming fungi............................63 63

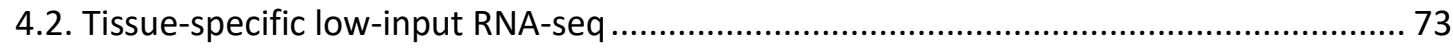

4.2.1. General description of the low-input RNA-seq workflow...................................... 73

4.2.2. Modifications and adjustments on the RNA-seq workflow .................................... 73

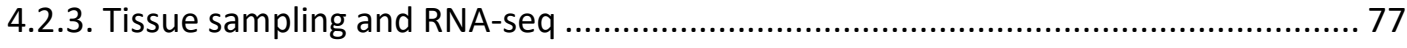

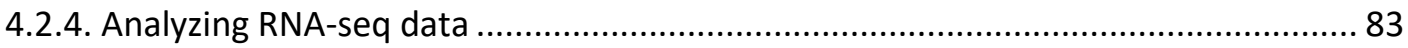

4.2.5. Differentially expressed genes at the transition from vegetative mycelium to the

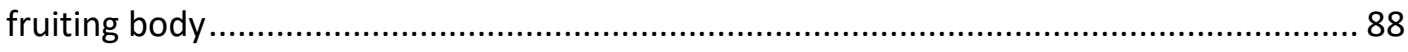

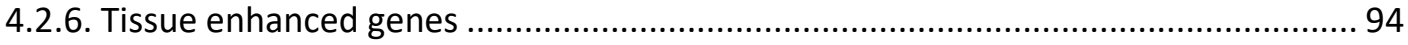

4.2.7. Genomic phylostratigraphy .............................................................................. 104

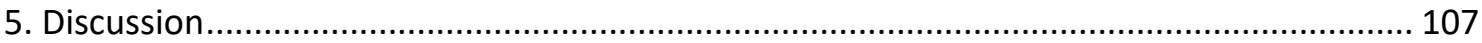

5.1. The evolution of complex multicellularity in fungi is incompletely known ................... 107

5.2. Reconstructing the evolutionary relationships within the Agaricomycetes based on

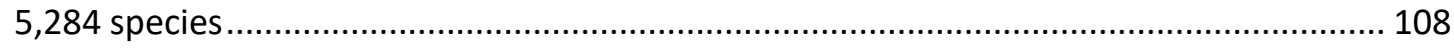

5.3. Macro-evolutionary patterns among mushroom-forming fungi .................................. 110

5.3.1. Diversification rate increase in the Jurassic period................................................. 110

5.3.2. Mass extinction events could follow different patterns than in other organisms . 110

5.3.3. Increased diversification rate is in concordance with the evolution of complex

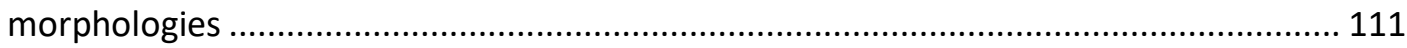

5.3.4. Five morphological traits with a positive effect on the diversification of mushroom-

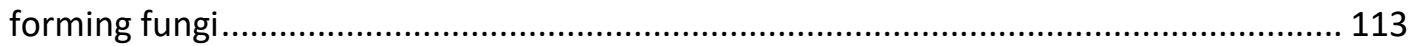

5.4. Developing a low-input tissue-specific RNA-seq workflow........................................... 116

5.4.1. Dissecting the early development of $C$. cinerea .................................................... 116

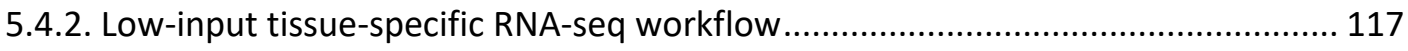

5.5. Transition from vegetative mycelium into hyphal knot involved active changes in the expression of genes related to hyphal growth patterns and cell wall remodeling ............... 118

5.6. Detecting tissue enhanced genes can help to understand the evolution of morphological

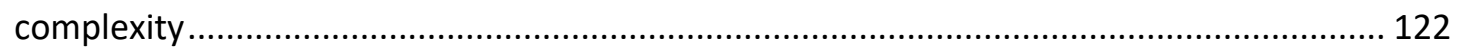

5.6.1. Tissues close to the environment express defense related and surface coating genes

5.6.2. Both genetic predisposition and derived genes could drive the evolution of

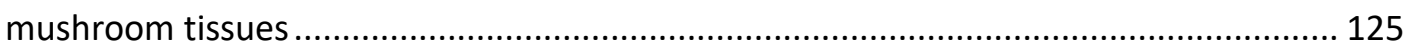

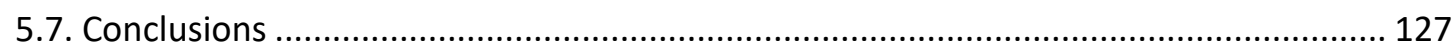

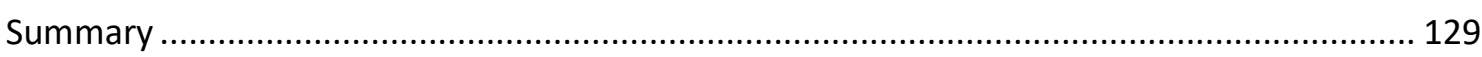

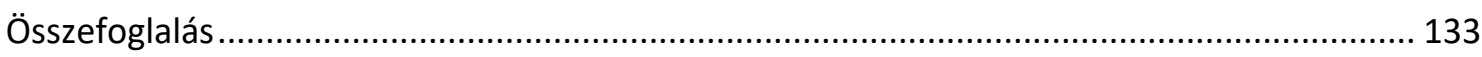


Acknowledgment

References.

Appendix

Appendix 1. Low-input RNA-seq protocol.

Appendix 2. Cell division, hyphal growth pattern and cell wall remodeling genes among H1 and $\mathrm{H} 2$ upregulated genes. 163

Appendix 3. $\mathrm{H} 1$ and $\mathrm{H} 2$ upregulated genes related to beta-glucan metabolism..... 


\section{List of Abbreviations}

ASR - Ancestral Character State Reconstruction

BAMM - Bayesian Analysis of Macro-evolutionary Mixtures

BD - Birth-Death

BF - Bayes Factor

BH - Benjamini-Hochberg

BiSSE - Binary State Speciation and Extinction

$\mathrm{CM}$ - Complex multicellular

CoMET - Compound Poisson process on Mass-Extinction Times

CPP - Cerato-platanin protein

DE - Differentially expressed or differential expression

DEPC - Diethyl pyrocarbonate

ECM - Ectomycorrhiza

ESS - Effective sample size

FB - Fruiting body

GH - Glycoside hydrolase

GLM - Generalized linear model

GO - Gene Ontology

GPI - Glycosylphosphatidylinositol

H1 - Primary hyphal knot

H2 - Secondary hyphal knot

IPR - InterPro

JGI - Joint Genomic Institute

J-K - Jurassic-Cretaceous

KEGG - Kyoto Encyclopedia of Genes and Genomes

KOG - EuKaryotic Orthologous Groups

K-Pg - Cretaceous-Paleogene

LCM - Laser capture microdissection

LFC - Logarithmic fold change

LPMO - Lytic polysaccharide mono-oxygenase

LRT - Likelihood ratio test

LTT - Lineages through time 
Ma - Million years

MBA - Histological stain: $1 \%$ Methylene blue, $1 \%$ borax and 1\% Azure II

MCMC - Markov chain Monte Carlo

ML - Maximum Likelihood

MRCA - Most recent common ancestor

MuSSE - Multi-State Speciation and Extinction

nrLSU - Nuclear large ribosomal subunit

P0 - Stage 0 primordium

P1 - Stage 1 primordium

P2 - Stage 2 primordium

PBS - Phosphate-buffered saline

PCA - Principal component analysis

PCD - Programmed cell death

PCM - Phylogenetic comparative methods

PI - Primordium initial

PRANK - Probabilistic Alignment Kit

PS - Phylostratum

PV - Partial veil

RBH - Reciprocal best hit

RF - Robinson-Foulds

RIN - RNA integrity number

rjMCMC - Reversible jump Markov chain Monte Carlo

rpb2 - RNA polymerase II second largest subunit

SM - Simple multicellular

SSE - State-dependent speciation and extinction

STRIPAK - Striatin-interacting phosphatases and kinases

tef1- $\alpha$ - Translation elongation factor $1-\alpha$

$\mathrm{TF}$ - Transcription factor

UV - Universal veil

VM - Vegetative mycelium 


\section{Introduction}

\subsection{Multicellularity is a widespread evolutionary innovation}

Multicellular organisms are essential components of life on Earth and thrive in all biogeographic realms (Knoll and Hewitt, 2011). Multicellularity has arisen in both prokaryotic and eukaryotic lineages at least 25 times, forming simple aggregates or colonies of cells, which is also called simple multicellularity by many authors (Knoll, 2011; Knoll and Hewitt, 2011). Numerous organisms reached a higher level of complexity, forming an endless variety of forms. These living things, also known as complex multicellular (CM) organisms, differ from simple multicellular (SM) organisms in that they develop into a three-dimensional organization, and only some of their cells are in direct contact with the environment (Knoll, 2011). CM organisms evolved relatively late in the history of life. The first living organism could arise around 3.7 billion years ago (Ohtomo et al., 2014), while the first sign for complex multicellularity is dated back to the Ediacaran Period (635-541 Ma ago, Knoll, 2011). This event could be a major evolutionary transition (Szathmáry and Maynard Smith, 1995) which had crucial impact on the present ecosystems.

Complex multicellularity evolved exclusively in eukaryotes in five main lineages from which three dominate the present terrestrial ecosystems: animals, embryophytic land plants and fungi (Knoll, 2011; Szathmáry, 2015; Nagy et al., 2018). All these lineages had to overcome evolutionary hurdles connected to multicellular life, which resulted in the evolution of effective cell adhesion mechanisms, efficient communication between cells and a coordinated developmental program, which allows the formation of complex morphologies. In animals, special adhesive molecules have evolved, such as cadherins, lectins, integrins but junctions between cells play a role in adhesion as well (Acharya and Yap, 2016; Hines and Taneyhill, 2019). Plant cells usually stayed in a fixed position after cell division, which is mainly accomplished by producing a pectin rich middle lamella (Daher and Braybrook, 2015). Among fungal species, the adhesins are the best-studied molecules to which adhesive functions were attributed (Lipke, 2018). The effective communication between adjacent cells was achieved with not only receptors but more or less continuous connections through plasmodesmata of plants, gap junctions of animals or pores of fungi (Knoll, 2011; Nagy et al., 2018). 
Information on the genetics and molecular background of multicellularity has been accumulated in the previous decades. However, the driving force of the evolution of complex multicellular life is still obscure. One hypothesis is based on the changes in the feeding mode of eukaryotes (Knoll and Lahr, 2016), supported by the fact that none of the complex multicellular organisms can be found within a phagotrophic clade. Another hypothesis state that the increase in atmospheric oxygen level could trigger the evolution of complex multicellular organisms (Knoll, 2011). Indeed, animal diversification shows a correlation with oxygen levels inferred from geochemical examinations. Nevertheless, it is more plausible that $\mathrm{CM}$ is driven by both biotic and abiotic factors.

The genetic background of the evolution of complex multicellularity could root in several mechanisms as well. The use of new generation sequencing methods (Ruiz-Trillo et al., 2007), were a great help to unravel some of these mechanisms. For example, in plants and partially in animals, one of the primary drivers could be the whole-genome duplication (Lang and Rensing, 2015). Pieces of evidence are also mounting that the evolution of multicellular organisms was driven by exaptation (Gould and Vrba, 1982). In contrast to adaptation, this evolutionary mechanism describes the process when traits (e.g., feathers) were adapted to functions in ancestors (e.g., insulation), but their role in the descendants are different (e.g., improved flying), that enhance the fitness of organisms. This mechanism can be easily applied to a pre-existing genetical toolkit of ancestors, which can give new adaptive features for descendants. Conserved gene circuits could explain the convergent evolution of similar morphologies (i.e., latent homology). For example, the symbiotic $\mathrm{N}_{2}$-fixation in angiosperms (Werner et al., 2014) or yeast lifestyle in fungi (Nagy et al., 2014) could be driven by latent homology. Convergence could also exist on molecular level, as it was revealed between Pezizomycotina and Agaricomycotina in fungi (Merényi et al., 2020) or different three-spined stickleback fishes (Colosimo et al., 2005).

\subsection{Complex multicellularity in fungi}

\subsubsection{The evolution of complex multicellularity in fungi}

The fungal kingdom is one of the five main groups where complex multicellularity evolved (Knoll, 2011), but within fungi, complex multicellularity could have convergently appeared at least eight times (Nagy et al., 2018). Most of the CM organisms can be found in two prominent clades, Pezizomycotina (Ascomycota) and 
Agaricomycotina (Basidiomycota) and a minority of them sporadically evolved in at least seven lineages: Mucoro-, Mortierello-, Glomero-, Taphrino-, Puccinio- and Ustilaginomycotina. The latter lineages primarily contain SM organisms or yeasts, and only a few species are capable for producing complex multicellular structures.

The convergent origins of $\mathrm{CM}$ structures among fungal lineages implies the uniqueness of the complex multicellularity in fungi. One of the main differences to other organisms could be that fungal complex multicellular structures are bound to a particular life period (fruiting bodies) or circumstances (mycorrhizae, rhizomorphs), and these are only a part of the whole organism. In contrast to this, the whole individual is the complex multicellular entity in other CM organisms (Nagy et al., 2018). Evolutionary innovations, which led to complex multicellularity, could follow each other with a different order in fungi and other organisms. (Nagy et al., 2020). The hyphal nature of fungi implies that the elongation, compartmentalization, communication, differentiation and adhesion was the order of innovations towards fungal multicellularity, while in animals or plants, adhesion could be the first leap and then machinery for communication and differentiation evolved. The relatively late evolution of mechanisms for cell adhesion in fungi is supported by adhesion-related protein families having undergone a small contraction in the ancestors of early diverging fungi (phlya outside Dikarya (Berbee et al., 2017)). However, later these families expanded in the Agarico- and Saccharomycotina clades (Kiss et al., 2019). Most of these adhesive proteins, including adhesins and lectins found to be glycosylphosphatidylinositol (GPI) anchored and to have a role in forming CM structures (Nagy et al., 2018).

Complex multicellular structures in fungi can vary in both size and level of complexity from vegetative to asexual and sexual reproductive structures. Miniature (1$2 \mathrm{~mm}$ to $1-2 \mathrm{~cm}$ ) asexual fruiting bodies are developed by many species in Ascomycota but vegetative CM structures (e.g., mycorrhizae, rhizomorphs, sclerotia) can be found among most of the dikaryotic species. Possibly, the most common fungal CM structures are the sexual fruiting bodies (FBs), whose primary purpose is to produce meiotic spores in a protective environment and facilitate spore dispersal (Moore et al., 2011; Nagy et al., 2017). To fulfill these functions, various cells and fungal tissues evolved, which makes fruiting bodies the most complex multicellular structures in fungi. The class Agaricomycetes stands out from fruiting body forming groups with its more than 20,000 species (Hibbett et al., 2014). 
Agaricomycetes, also called mushroom-forming fungi, contains 20 orders with numerous ecologically and economically important species. Out of the 20 orders, the best known are the Agaricales and Boletales, where the most prominent agaricoid and boletoid species (mushrooms with cap and stipe in the respective taxa) can be found, such as the button (Agaricus bisporus) or porcini mushroom (Boletus edulis). Mushroom-forming fungi have various ecological functions (Hibbett et al., 2014). The majority of the ectomycorrhizal species can be found here, but orchid and insect symbionts exist as well. Moreover, lichen-forming species, endophytes and bryophilous species can be found among Agaricomycetes. Many mushrooms are plant pathogenic, mycoparasite or nematode trapper and contribute to the healthy functioning of ecosystems. In this class, new enzymes evolved, which endowed a group of mushrooms (white rots) with the ability of lignin degradation (Floudas et al., 2012; Naranjo-Ortiz and Gabaldón, 2019). Therefore, saprotrophs in Agaricomycetes are essential parts of the present ecosystems.

Besides their importance in ecological functioning, mushroom agriculture was valued to $\$ 63$ billion in 2013 (Royse et al., 2017), which is a comparable impact on the world economy with the wheat production in the same year ( $\$ 185$ billion). Apart from the food industry, mushroom-forming fungi could give alternative ways for the extremely polluting fashion industry (Wojciechowska, 2017; Bustillos et al., 2020) or to packaging and building materials (Girometta et al., 2019; Jones et al., 2020). Moreover, mushrooms have a tradition in alternative medicine, which recently started to be recognized in western medicine (Sullivan et al., 2006; Gargano et al., 2017).

\subsubsection{The morphological complexity of mushroom-forming fungi}

Based on macro-morphological traits, the simplest mushrooms are flattened or crust-like so-called resupinate types, but more complex forms such as clavarioid (clubshaped), coralloid, or cyphelloid (cup-shaped) morphologies formed in various groups (Hibbett et al., 2014). The most complex mushroom morphology is called pileate-stipitate type, also known as toadstool morphology, which consists of a stipe and a cap (Hibbett, 2007). Gasteroid mushrooms, where the spores are produced internally, can be evolutionarily derived from the pileate-stipitate morphology (Hibbett, 2004; Wilson et al., 2011).

These highly morphologically variable fruiting bodies have something in common. All of them have a spore-bearing surface, in other words, a hymenophore, where 
spore production takes place. In the case of simple mushroom morphologies (resupinate, clavarioid, coralloid), the hymenophore can be found on the surface of the mushrooms, and it spans at the underside of the cap or inside the fruiting body of the pileate-stipitate or the gasteroid mushrooms, respectively (Clémencon, 2012). Morphological diversity can be observed on the hymenophore as well: smooth surface, warts, spines, veins, pores and gills. Clémencon classified hymenophores into 11 groups by complexity and the relative size of the hymenophore (Clémencon, 2012). The importance of the structured hymenophore is that it can produce more spores by increasing the surface/mass ratio (Fischer and Money, 2010). It was also demonstrated that among three main structured hymenophore, spines harbor the smallest relative surface area, which was followed by pores, then gills (Clémencon, 2012; Halbwachs et al., 2016). The shape and the structure of the hymenophore could have been driven by other factors than solely the number of spores developed. For example, cystidia produced on gills of two pileate-stipitate species (Strobilurus ohshimae and Russula bella) were destructive for fungivorous collembolans (Nakamori and Suzuki, 2007). The mechanism of active basidiospore discharge includes the secretion of a droplet of fluid ("Buller's drop"), which contributes to the surfacetension catapulting of spores (Moore et al., 2011). Therefore structured hymenophores could ease the production of Buller's drops by providing a micro-environment with high humidity (Halbwachs and Bässler, 2015)

Spore production is a central and essential part of the fungal life-cycle, which have role in sexual and asexual reproduction. Therefore, protective mechanisms of mushroom development should have evolved which provide a safe environment for spore production or give protection against biotic and abiotic impacts. Cap development could have been adaptive for species from many aspects. For example, it can provide a massive base for hymenophore development and orient the hymenophore to have an optimal airflow for spore release (Dressaire et al., 2016). Furthermore, the cap tissue of many species contains ultraviolet protecting pigments or other substance, which makes cap surface viscid and resistant to desiccation (Halbwachs et al., 2016). It is also showed that many species produce bactericide, nematotoxic or entomotoxic materials (Künzler, 2018). Therefore, it may be hypothesized that one of the most widespread solutions of protecting spore development is to develop a cap that can give protection from both abiotic and biotic impacts from above (Halbwachs et al., 2016). In addition to the cap, many mushroom species produce mycelial sheaths, which enclose the developing FB (i.e., primordium) or 
the immature hymenophore. The universal veil, which envelopes the outer surface of the primordium, can be present from the beginning of mushroom development and can persist until spore release. Remnants of the universal veil of mature fruiting bodies can be recognized as patches, scales, dots on the cap or volva on the stipe. The partial veil covers the surface of the hymenophore and can be persistent until spore maturation (Watling, 1985; Clémencon, 2012). The ring on the stipe is the typical remnant of the disrupted partial veil. These protective veils form not just a physical barrier against environmental impacts but produce chemicals (secondary metabolites, peptides, and proteins) against bacteria or fungivorous animals (mammals, arthropods, nematodes etc.) (Künzler, 2018). For example, galectins, which are effective nematotoxic and entomotoxic proteins (Bleuler-Martínez et al., 2011; Sabotič et al., 2016) have high expression in the velum of the cap during the development of Coprinopsis cinerea (Boulianne et al., 2000).

The presence of the universal or partial veil received much attention in mycology because agaricoid and boletoid mushrooms are frequently classified based on these traits. Reijnders classified agaricoid and boletoid mushrooms into two main categories: gymnocarpous and angiocarpous. The hymenophore is not covered with any veil or sheath in the gymnocarpic mushrooms. In contrast, angiocarpic taxa always bear, at least in the primordium stage, a protecting veil (Reijnders, 1948; Clémencon, 2012). Reijnders further described eleven subcategories within gymnocarpic and angiocarpic species, which was later followed by other authors (Reijnders and Moore, 1985; Moore et al., 2011). In contrast to this, Clémencon, inspired by George F. Atkinson's (1854-1918) works, classified agaricoid and boletoid mushrooms based on the spatial and temporal position of internal tissues during the development of the fruiting body (Clémencon, 2012). He distinguished epinodular and endonodular development as the two main categories. These terms are based on the nodulus, which is a loose composition of undifferentiated hyphae. The nodulus can be initiated from the vegetative mycelium or rhizomorph, and it is developmentally determined towards fruiting body, sclerotium or termitosphere (Clémencon, 2012). In the case of epinodular mushrooms, a shaft (upwardly growing mass of hyphae) grows out from the nodulus on which the cap is apically formed. No shaft tissue exists in endonodular species, therefore every main tissue type (cap, stipe, lamellae) develops inside the nodulus. In both cases, veils can present, but at least eight names were dedicated to them (universal veil: archiblema, lemmablema, 
ectoblema, teleoblema, bulboblema; partial veil: cleistoblema, lipsanoblema and mesoblema) depending on the origin of the tissue (in detail see Clémencon, 2012).

In this study, we use the term 'fungal tissue' which requires some explanations. Historically fungal hyphal aggregates or any fungal context that is developmentally determined to an extent is called plectenchyma (Moore, 1995; Clémencon, 2012). Despite the existence of the term plectenchyma, the fungal tissue phrase is still widely used by many authors (Moore, 1995; Kües and Navarro-González, 2015). In the case of animals and plants, a tissue is defined as a group of similar cells performing specialized functions (Cowin, 2000; Evert and Eichhorn, 2006). Also, extracellular matrix between animal cells is a frequent feature that is used to characterize tissue. Generally, two main types of plectenchyma were described. The individual hyphae can be recognized in the prosenchyma, but the hyphal nature is barely or not noticeable in the pseudoparenchyma. Therefore, pseudoparenchyma resembles the tissues of animals and plants and can be found in most of the complex multicellular structures, such as ectomycorrhiza, sclerotium or fruiting body (Kües, 2000; Agerer, 2006). Moreover, filamentous like structures exist in both plants and animals, which can form tissues. For example, fern spores germinate into a filament of cells, but at some point, the apical cell started to divide by parallel cell walls to the long axis. In contrast, fungal hyphae never produce parallel septa to the longitudinal axis, but they change the growth pattern by forming new branches (Moore, 1995). Animal axons can perform apical growth and precisely find their target regions, to which an extreme example is the $\sim 50 \mathrm{~mm}$ long axons of the retinal ganglion cells (Mann et al., 2004; Yu et al., 2013).

Nevertheless, fungal hyphae have a different structure and behavior than most of the plant or animal cells, yet they can fulfill all the functions of a conventional cell. In addition, extracellular matrix or gel-like matrix is produced in fungi (Riquelme et al., 2018). Polysaccharide rich extracellular matrix was observed in biofilms produced by Aspergillus fumigatus, Candida albicans, Cryptococcus neoformans and Saccharomyces cerevisiae (Reichhardt et al., 2015; Mitchell et al., 2016). A mucilaginous substance was also observed between hyphae of the developing fruiting body using transmission electron microscopy (van der Valk and Marchant, 1978). For the sake of simplicity and because a mass of fungal hyphae frequently shows similarity with animal and plant tissues, we use the term fungal tissue for every group of cells or hyphae which show distinct anatomical features. 


\subsection{Macro-evolution of mushroom-forming fungi}

\subsubsection{The macro-evolution of mushrooms}

As it is described above, Agaricomycetes is both morphologically and ecologically diverse group. Complex fruiting body types can be found in several independent lineages (Hibbett, 2007) and mushrooms a have pivotal role in ecosystem functioning including degradation of plant material (lignin decomposition) or symbiotic relationships. (Naranjo-Ortiz and Gabaldón, 2019). However, how this diversity evolved and what major evolutionary events led to the present state of Agaricomycetes, is barely known.

One of the main hurdles to examine the macro-evolution of mushroom-forming fungi is the scarcity of fossilized specimens. Most of the fossils were dated to the Neogene $(23.03-2.58 \mathrm{Ma})$ or Paleogene ( $66-23.03 \mathrm{Ma})$ periods. Many of them bear poroid hymenophores resembling the present species of Ganoderma, Fomes, Polyporus or Trametes (Chaney et al., 1936; Brown, 1940; Smith et al., 2004) or have gasteroid forms (Magallon-Puebla and Cevallos-Ferriz, 1993; Poinar, 2014). However, most of the order level clades could have evolved in the Cretaceous (145 - 66 Ma) or older periods (Floudas et al., 2012; Kohler et al., 2015), therefore relatively young fossils are not informative for inferring class wise dates and evolutionary patterns. Only a few mushroom fossils were found from the Cretaceous period. One of the oldest mushroom fossils could be the Quatsinoporites cranhamii with a poroid hymenophore, which was found among 118113 Ma old rocks in Canada (Smith et al., 2004). Two agaricoid fossils were dated to the Cretaceous as well. The Archaeomarasmius leggettii preserved in amber and showed the morphology of a marasmioid species (Hibbett et al., 1997). Palaeoagaracites antiquus was dated to around $100 \mathrm{Ma}$ ago and was assigned to the Agaricales clade (Poinar and Buckley, 2007).

The amount and the quality of mushroom fossils are insufficient to examine the evolutionary patterns of Agaricomycetes. However, few studies were conducted to infer macro-evolutionary patterns of lineages in mushroom-forming fungi by using modern bioinformatic methods (see 1.3.2 paragraph) (Hibbett and Binder, 2002; Hibbett, 2004, 2007; Nagy et al., 2012). So far, it is revealed that the evolution of mushrooms could have started as a resupinate ancestor (Sánchez-García et al., 2020). Then, through a coralloid/clavarioid type, pileate-stipitate mushrooms convergently evolved (Hibbett and 
Binder, 2002; Hibbett, 2004, 2007). Gasteroid fungi could evolve from pileate-stipitate mushrooms, and both early and modern phylogenetic comparative studies showed that this is likely an irreversible morphological transition (Hibbett, 2004; Sánchez-García et al., 2020).

The trend of convergent evolution towards pileate-stipitate mushrooms and the prevalence of this morphology implies that the toadstool morphology can bear traits which are evolutionarily favorable for species. For example, it was hypothesized that the driving force of fruiting body evolution is the effective spore dispersal (Hibbett and Binder, 2002), but to our knowledge, it has not been tested yet.

Bioinformatic methods have been developed actively to infer diversification rates (speciation minus extinction) and connect it to a certain trait. The nutritional modes of species were examined in different mushroom lineages by many authors. The positive effect of ectomycorrhizal (ECM) lifestyle was indicated among species in the clades of Laccaria, Tricholoma, Entoloma and Boletaceae (Sánchez-Garcia et al., 2017; Wilson et al., 2017; Sato and Toju, 2019). In a recent study, both the nutritional modes (ECM, brown rot or white rot) and fruiting body morphologies were examined in the whole Agaricomycetes involving more than 8,000 species (Sánchez-García et al., 2020). They found that morphological innovations could have more effect on the diversification of species than nutritional modes. Apart from this study, the mushroom morphology received less attention in trait dependent diversification analyses. Wilson et al. 2011 showed that the diversification of species with gasteroid fruiting bodies was higher than that of non-gasteroid species in the Boletales, Phallomycetidae or Lycoperdaceae clades (Wilson et al., 2011).

The scarcity of analyses on the macro-evolution of mushrooms urged this study to thoroughly examine the diversification and macro-evolution of Agaricomycetes, including traits which could facilitate the success of spore production by supplying protection (cap or protecting veils) or increase the number of spores by expanding the surface area of the hymenophore.

\subsubsection{The background of phylogenetic comparative methods used in this study}

In most of the main lineages, fossil records are scarce; therefore, reconstructing their evolutionary history is problematic. However, thanks to the bioinformatic advances 
which have been made in previous decades, phylogenetic comparative methods (PCMs) can hand scientists new tools (Cornwell and Nakagawa, 2017), which, with considerable care and sufficient precaution, can supply information on the macro-evolution of various organisms. In this study, we used some of the modern, recently developed PCMs, which can answer two groups of questions. One is the "tempo and mode of the evolution" (Cornwell and Nakagawa, 2017), in other words, the speed of which changes appear along the phylogeny and the manner (e.g., gradual, episodic) as it happens. The other question is how phenotypic traits have been changed through evolution. To answer these questions, PCMs use the phylogenetic tree as the reconstructed evolutionary history of species under scrutiny.

To examine the "tempo and mode of the evolution", one of the options is to model the frequency of the speciation events. The simplest way to do that is to depict the number of lineages in a phylogeny through time, which is also called lineages through time (LTT) plot (Nee et al., 1995). A more sophisticated method if the birth of new lineages is modeled so biased sampling and hidden birth events can have a low effect on the results. The pure birth process is called the Yule model, which can give valuable information on the birth rate (i.e., speciation rate) of every branch in the phylogeny. Both the Speciation (birth) and extinction (death) rates can be simultaneously modeled by the birth-death (BD) process using information such as branch length and branching patterns of a timecalibrated tree, in other words, chronogram (Figure 1. A and B) (Yang, 2014). This model is useful to infer the diversification rate (speciation minus extinction rate) (Figure 1. C), which can describe e.g., whether a linage went through an expansion (high positive diversification rate) or reduction (low negative diversification rate). Several extensions of the BD model have been made so far. Some extended models infer events with a shift in speciation or extinction rates, representing a presumed key innovation event (Alfaro et al., 2009; Rabosky, 2014). Bayesian analysis of macro-evolutionary mixtures (BAMM) can infer evolutionary rates through time and among clades while it detects rate shifts without a priori specifications (Rabosky, 2014). BAMM is based on a Bayesian analysis using reversible jump Markov chain Monte Carlo (rjMCMC), which can explore a vast number of evolutionary scenarios and automatically detect and test the most plausible evolutionary histories. We know from fossil records that not just key innovation events, but mass extinction events affected species and shaped the history of life. The compound Poisson process on Mass-Extinction Times (CoMET) model intended to infer and test 
events along the tree when the majority of species went extinct (Höhna, 2015; May et al., 2016). The CoMET model is a powerful tool to detect traces of mass extinction events in the phylogeny of species (Arcila and Tyler, 2017; Ribeiro et al., 2018; Condamine et al., 2020).
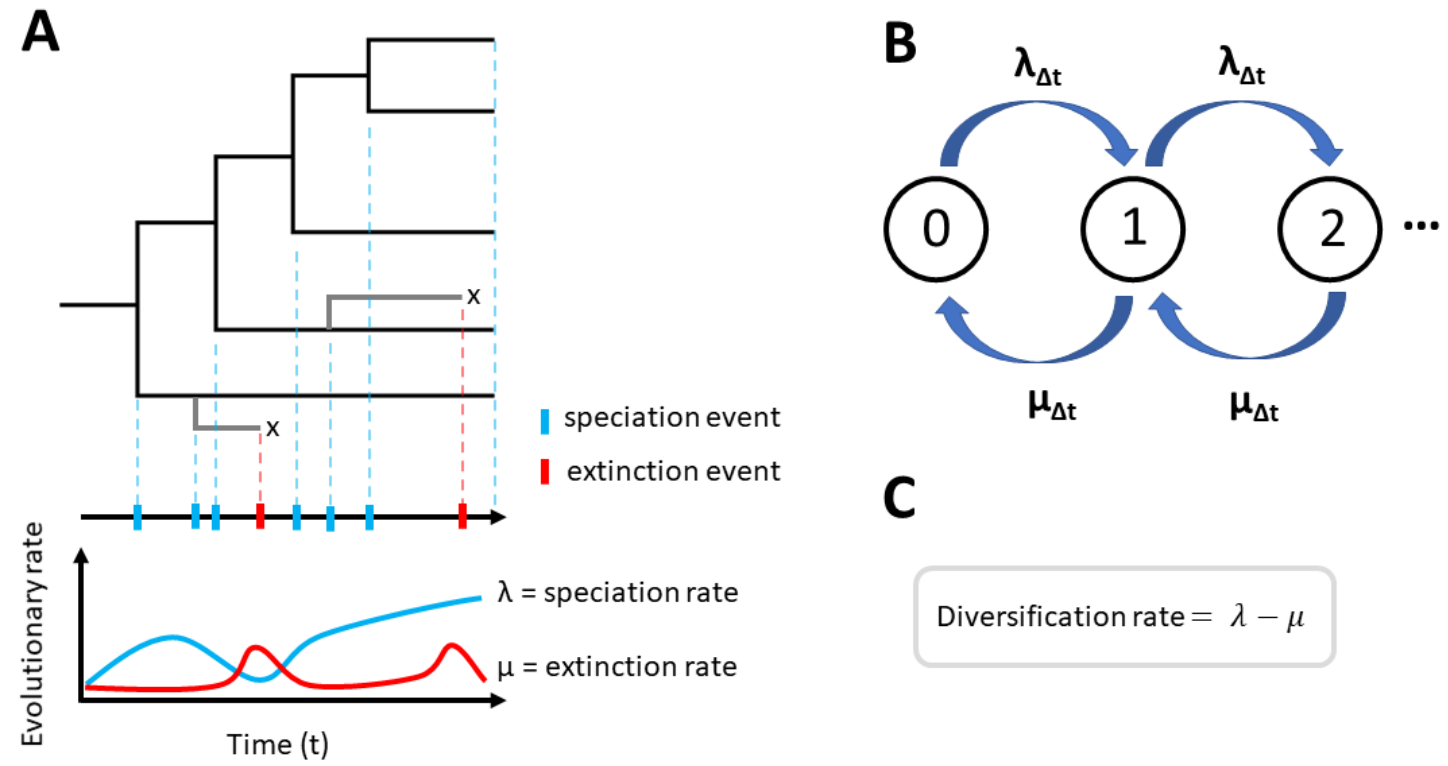

Figure 1. Schematic figures on the Birth-Death process and the estimation of the diversification rate. (A) Time-calibrated tree (black lines) with some lineages went extinct (grey lines ended in $x$ ). Each branching event corresponds to a speciation event (blue dashed lines and sticks), and extinct lineages correspond to extinction events (red dashed lines and sticks). Speciation and extinction events can be transformed into speciation and extinction rates, respectively. These evolutionary rates can be plotted through time to depict the tempo and mode of evolution. (B) Birth-death process. BD model is a special case of a Markov model because the future state depends on the current state and not on events that happened before but, the value of the state (in the BD model, the number of lineages) can be increased or decreased only by one. The figure represents the states (number of lineages) of the BD model at time $t . \lambda_{\Delta t}$ and $\mu_{\Delta t}$ stand for the speciation and extinction rate in time $t$, respectively. $(\boldsymbol{C})$ The diversification rate can be calculated by extracting the extinction rate from the speciation rate.

Trait evolution is also a central question of PCMs (Figure 2. A). One can infer character state transition rates and trait dependent diversification rates by combining the information of a phylogenetic tree and contemporary data (Figure 2. B and D). Discrete and continuous data or traits can be modeled with different algorithms, but in the following, we are going to focus on the analysis of discrete morphological characters (Pagel, 1994), which is more relevant to this study. The most basic model that can be used for discrete characters is called the Mk model (Lewis, 2001), which is a Markov model with k states observed (Figure 2. C). The simplest scenario is when one trait is examined 
with two possible states (e.g., viviparity or oviparity), so the evolutionary history of traits can be inferred by fitting a two-parameter Mk model to the data (Figure 2. B). The transition rates ( $\mathrm{q}_{\mathrm{ab}}$ or $\mathrm{q}_{\mathrm{ba}}$ on Figure. 2) give information about evolutionary trends. For example, higher transition rates towards state $b\left(\mathrm{q}_{\mathrm{ab}}\right)$ relative to the rate of the reverse direction ( $\mathrm{q}_{\mathrm{ba}}$ ) imply that state $\mathrm{b}$ could be an evolutionary favorable character state since it frequently appeared through evolution. The Mk model can be combined with the BD model, and one can infer trait dependent diversification rates, that is, speciation and extinction rates of lineages with a particular character state (Figure 2. D and E). The simplest of these models is called the binary state speciation and extinction (BiSSE) model (Maddison et al., 2007). Using Mk or BiSSE model the evolution of a character can be inferred and the character states of ancestors can be reconstructed. This process is called ancestral state reconstruction (ASR) and can be useful to uncover the evolutionary pattern of a character (Bollback, 2006; Holland et al., 2020).

A

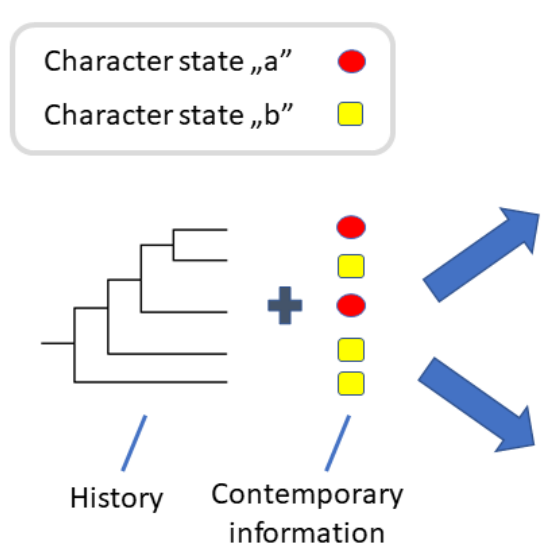

B

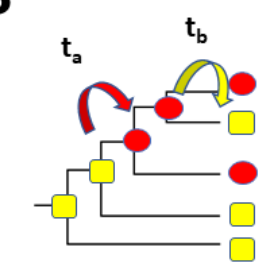

D

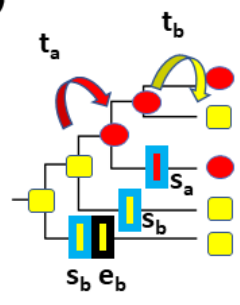

C

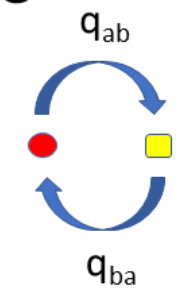

E

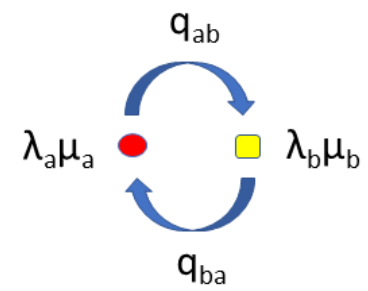

Figure 2. Schematic figures of PCMs examine trait evolution. (A) Combining the history of species (e.g., phylogenetic tree) and contemporary data (e.g., categorical morphological character) and infer the evolutionary history. (B) Inferring character state transition. $t_{a}$ and $t_{b}$ represent transition events from state $b$ to state $a$ and from state a to state $b$, respectively. (C) $M k$ model with $k=2$ states. $q_{a b}$ and $q_{b a}$ represent the transition rate from the state a to the state $b$, and from the state $b$ to state $a$, respectively. (D) Inferring trait dependent diversification rate. $s_{b}$, $s_{a}$ and $e_{b}$ represent a speciation event for lineages in state $b$, state $a$, and an extinction event for lineages in state b, respectively. $(\boldsymbol{E})$ The BiSSE model. It is similar to the Mk model, but a BD process is built in it; thus the speciation $\left(\lambda_{a}, \lambda_{b}\right)$ and the extinction $\left(\mu_{a}, \mu_{a}\right)$ rates are inferred for lineages are in state $a$ and state $b$, respectively. 
All PCMs rely on a phylogeny, which represents the history of every species of the lineage examined. Therefore, it is crucial to infer a robust phylogeny, and, in most cases, it is necessary to include all contemporary species into the analysis (Fitzjohn et al., 2009). Many PCMs assume that branch length is proportional to time. Therefore, time calibration of phylogeny could be essential in many cases. The time calibration of trees started with the molecular clock concept, which posits that the timing of the evolutionary divergence can be inferred by assuming a constant rate of genetic changes across lineages (Ho and Duchêne, 2014). However, it turned out that the rate of changes is not constant through time or across lineages, which urged scientists to relax the molecular clock (Lemey et al., 2009). The underlying problem of the relaxed molecular clock analyses is that branch length is the product of the time and the evolutionary rate, which needs to be resolved. Two of the most widely used methods to relax the molecular clock are the rate smoothing and parametric methods (Ho and Duchêne, 2014). Rate smoothing methods are based on the idea that rates show a certain degree of autocorrelation since closely related lineages could share similar life-history and environmental factors. Therefore, these methods try to smooth the rates between adjacent branches (Sanderson, 2002, 2003). One of the methods is to infer uncorrelated relaxed clocks is to implement parametric methods in a Bayesian framework and jointly estimate parameter-rich models such as substitution and tree models or models of rate variations across lineages (Bromham et al., 2018).

Modern bioinformatics tools apply two primary methods the assess model fit (Lemey et al., 2009): maximum likelihood (ML) and Bayesian methods. ML tries to find one best model by finding the set of model parameters that maximize the likelihood function using heuristic search. In contrast to ML, where a point estimate is obtained, Bayesian methods coupled with Markov chain Monte Carlo (MCMC) method generate a sample from the posterior, which can be used to evaluate statistics that describe the robustness and variance of the inferred parameters (Nascimento et al., 2017).

Finally, both ML and Bayesian frameworks are suitable to perform model tests to examine alternative evolutionary scenarios (Pagel, 1994). The likelihood ratio (LR) test is used to compare the maximized likelihood values of two models. The LR value defines the twice of the ratio of the logarithmic likelihoods of the two models. The LR follows a chi-squared distribution from which p-value can be obtained or, as a rule of thumb, LR is higher than four could be considered a significant difference (Pagel, 1999). In a Bayesian 
framework, a model test can be performed with a similar logic to the LR but using the marginal likelihoods of the two models resulting in a Bayes factor of the test (Yang, 2014).

\subsection{The genetic background of mushroom development: the model organism Coprinopsis cinerea}

\subsubsection{Fruiting body development of C. cinerea}

Many physiological, anatomical and genetic studies have been carried out on the ontogeny of mushrooms (Moore et al., 2011; Clémencon, 2012; Kües and NavarroGonzález, 2015). Among them, coprinoid species received special attention because they could be easily grown in laboratory conditions and have a fast life cycle (1-2 weeks). One of the enigmatic features of coprinoid species that the mature spores are released through the autodigestion of the cap. The resulting blackish liquid provided the common name "inky cap" of these species. Inky caps are widespread saprotrophs and decaying various plant (e.g., wood debris, led litter) and animal (e.g., dung) materials. Among coprinoid species, Coprinopsis cinerea became a model mushroom, having a plethora of genetically modified mutants strains (Kües, 2000) and it was among the first fungal genomes which were sequenced (Stajich et al., 2010). Thanks to genetic and bulk RNA-seq analyses of C. cinerea, our knowledge on the genetic background of mushroom development have been further widened (Plaza et al., 2014; Muraguchi et al., 2015; Krizsán et al., 2019; Xie et al., 2020).

C. cinerea is an endocarpic mushroom based on Clemencon's classification or a bivelangiocarpic one sensu Reijnders (Reijnders, 1979; Watling, 1985; Clémencon, 2012). Regarding the ontogeny of $C$. cinerea we follow the developmental description of Kües and Navarro-González, who reviewed and aligned the description of developmental stages made by several authors in the previous hundred years (Kües and NavarroGonzález, 2015). Kües and Navarro-González delineate eight developmental stages, including two hyphal knot stages, five primordial stages and the mature fruiting body. All of these stages are under light control and follow dark/light regimes. The development of C. cinerea starts with forming a nodulus sensu Clémencon, here it is called hyphal knot. The primary hyphal knot is the first stage, which is composed by undifferentiated shortly branched hyphae forming a three-dimensional ball-like structure around $0.1 \mathrm{~mm}$ in 
diameter. It is debated how many individual hyphae initiate the hyphal knot. There is an example of hyphal knot formation from a single hypha (Kües, 2000), but the controlled aggregation of number of adjacent aerial hyphae is more common (Henderson and Ross, 1983; Heckman et al., 1989; Kües, 2000). The hyphae of the primary hyphal knot soon compose a dense structure in the center, differentiating into a secondary hyphal knot with two tissue types, a denser inner part (nodulus) and the peripheric layer consist of loose inflated hyphae (primordial universal veil). The development of the secondary hyphal knot is also light controlled because sclerotium is formed in the absence of light exposure (Kües, 2000; Kamada et al., 2010). Blue light receptors, primarily the white collar complex (WCC), have a central role in light-sensing through fruiting body development (Kamada et al., 2010; Nagy et al., 2017) because WCC interacts with the mating-type genes through number of pathways, including MAPK (Nagy et al., 2018).

Several cellular changes happen during the transition from vegetative mycelium to the three-dimensional complex multicellular hyphal knots. Generally, three mechanisms can be linked to the regulation of the growth pattern of the mycelium: hyphal polarity, branch initiation and the spatial distribution of hyphae (Moore et al., 2011). For example, the avoiding reaction of hyphae within the vegetative mycelium changes to positive tropism towards each other (Moore, 1995; Brand and Gow, 2009). The branching events are also increased and changes in the septation pattern can be observed (Kües, 2000). Branching can be regulated by several factors and molecules, including cyclic AMP or cyclosporine or even heat-shock proteins (Moore et al., 2011). The pattern of septum formation could be controlled by several factors and have a significant effect on the compartmentalization of hyphae and the regulated flow of cytoplasm and organelles (Fricker et al., 2017). One of the main groups of proteins that play a role in septum formation is called septins, which is also in close contact with cell division and polarized growth (Khan, A. et al., 2015).

Cell fusion is widespread among eukaryotic species as it is in fungi (van der Valk and Marchant, 1978; Fleißner et al., 2008; Daskalov et al., 2017). Hyphal fusion is an intrinsic feature of the mycelium enabling the organism to efficiently perform longdistance mass flow (Fricker et al., 2017), which also increases the robustness of the mycelial network and allows a higher-level organization of the hyphae (Nagy et al., 2020). Several proteins could participate in hyphal anastomosis, including transcription factors, protein kinases (Fu et al., 2011) and the striatin-interacting phosphatases and 
kinases (SRTIPAK) complex. This complex consists of conserved eukaryotic proteins and recently discovered among fungi (Bloemendal et al., 2012).

The cell wall also goes through changes during the initial steps of the fruiting body development (Kamada and Tsuru, 1993). The random orientation of chitin microfibrils of hyphae in mycelium is changed to transverse orientation in hyphal knots. The chitin content is first decreased and then increased in hyphal knots relative to the vegetative mycelium, but the thickness of the cell wall was increased continuously during development. It was also showed that carbohydrate metabolism has an essential role in the initiation of the fruiting body (Brunt and Moore, 1989). Changes in expression of genes associated with glycogen metabolism were shown through the development of $C$. cinerea (Krizsán et al., 2019; Xie et al., 2020). Furthermore, the accumulation of glycogen in the basal region of the secondary hyphal knot was also observed (Matthews and Niederpruem, 1973; van der Valk and Marchant, 1978).

The next developmental stage is stage 1 primordium $(\sim 0.5 \mathrm{~mm}$ in diameter), where the cap and stipe rudiments first develop (Kües and Navarro-González, 2015). Formation of these tissues starts at the primordial shaft (apex of the primordium) in an isocarpous manner, that is, tissue development happens at more or less simultaneously. The basal tissue is called nodulus by many authors because it still shows a compact structure as the central region of the secondary hyphal knot (Clémencon, 2012). Stage 2 primordium becomes pearl-shaped with $1-2 \mathrm{~mm}$ in height. The hymenophore starts to develop in this stage in the form of small ridges and later, it progresses to primary gills that grow between the cap and a tissue layer called lipsanoblema. Lipsanoblema connects the cap margin and the stipe, and the partial veil may derive from this tissue (Moore et al., 2011; Clémencon, 2012). Initially, all the lamellae are attached to each other, but according to some authors, some of the cells go through programmed cell death (PCD), which separates the primordial gills (Lu, 1991). In subsequent stages, the stipe and cap elongation and the development of the secondary gills take place. Primordial development ends with stage 5 primordium, where the karyogamy and the meiosis happen in the basidia. The mature fruiting body goes through a fast stipe elongation and cap autolysis starts at the edges to release and disperse spores. The majority of previous studies examined well-developed primordial stages, therefore we have a good understanding of gill development and stipe elongation and many genes were connected to these mechanisms (Muraguchi and Kamada, 2000; Arima et al., 2004; Muraguchi et al., 2008; 
Moore et al., 2011; Shioya et al., 2013; Plaza et al., 2014; Kües and Navarro-González, 2015; Krizsán et al., 2019). However, the development of the main tissue types, which define the broad anatomy of the mature fruiting body, starts in stage 1 primordium or earlier. That is why we attempted to examine the early developmental stages of $C$. cinerea using laser-capture microdissection (LCM) technique and examine tissue-specific transcriptomics up to stage 2 primordium.

\subsubsection{RNA-seq analyses to examine the genetic background of morphological development}

In the following we would like to give a brief overview of some of the transcriptomic methods, to put into a context the low-input RNA sequencing method we used in this study. Transcriptomic analyses provided enormous information in the field of evolutionary developmental biology of most of the organism (Uhlén et al., 2016; Brunet and King, 2017; Sebe-Pedros et al., 2017; Krizsán et al., 2019). In previous years new sample isolation and RNA sequencing methods have been started to develop (Wolf, 2013; Saliba et al., 2014; Ziegenhain et al., 2017). The most promising techniques are single-cell based methods, where individual cells or groups of cells can be isolated to examine mRNA expression profiles with a high resolution (Saliba et al., 2014). Fluorescence-activated cell sorting, microfluidic-, or optofluidic-based cell handling methods could efficiently isolate hundreds or thousands of cells, but they cannot provide spatial information of the cells within the samples. In contrast to this, laser-capture microdissection (LCM) can isolate cells from histological sections. Therefore, spatial information is given by this method, but it is more work demanding and time-consuming than cell sorting methods. However, it has to be noted that state-of-the-art techniques, such as the Slide-seq can offer high throughput spatial RNA-seq analyses (Rodriques et al., 2019). The isolated cells or tissue samples contain an insufficient amount of RNA for sequencing. Therefore, methods were developed to amplify cDNA, which could naturally cause bias in expression level estimations. However, applying unique molecular identifier (UMI) or RNA spike-in (e.g., the product of the External RNA Controls Consortium (ERCC)) could help to identify or correct biases caused by cDNA amplification procedure. The methods could differ in whether they amplify the 3 prime of the mRNA (e.g., CEL-seq, Drop-seq, MARS-seq) or the full-length (Smart-seq, Smart-seq2) and in compatibility with different sample isolating methods. 
The core data of an RNA-seq analysis comes as read counts per genes, which has specific statistical attributes such as non-normality, the dependence of dispersion on the mean and the small number of replicates (Love et al., 2014). Some of these limitations can be overcome by pooling information across genes using likelihood or Bayesian models and estimating dispersion. A general question in RNA-seq analyses is whether the differences between average expression levels of different conditions are significant. The differences are usually measured by the logarithmic fold change (LFC) between two conditions and the significance can be inferred by statistical tests such as F-test, Waldtest or likelihood ratio test (Robinson et al., 2010; Love et al., 2014).

LFC can be used for ranking genes, however, low read counts or certain experimental conditions can cause high variation in LFC, which could compromise the interpretation of the results. One approach is to filter out low count genes, but setting an empirical threshold could cause the loss of relevant or interesting genes. Another approach is to perform Bayes modeling on the amount of shrinkage for fold-change estimation (Stephens, 2017). The idea behind these approaches is to shrink LFC estimates toward zero, but the amount of shrinkage depends on the available information for a gene (e.g., low read count or few degrees of freedom result in high shrinkage).

Statistical significance (P-values) is essential to assess differences between conditions. However statistical tests are performed on each gene resulting in thousands of tests, therefore p-value adjustment is needed to control false discovery rate (FDR). One standard method to overcome this multiple comparisons problem is the BenjaminiHochberg $(\mathrm{BH})$ procedure, which adjusts p-values by taking into account the number of tests performed (Benjamini and Hochberg, 1995). However, BH is suboptimal when the signal-to-noise ratio is different between individual tests, which is the case in RNA-seq analysis due to the vast differences in the number of mapped reads per gene. Therefore, covariates that do not affect the P-value but informative on the power or the prior probability of the test (e.g. sum of read counts per gene across all samples) can be used to apply weighted $\mathrm{BH}$ method improving the power of large-scale multiple testing (Ignatiadis et al., 2016). 


\section{Objectives of the thesis}

We aimed to investigate the macro-evolution of the largest class, the mushroomforming fungi (Agaricomycetes) in the fungal kingdom, which contains complex multicellular structures. We wanted to examine the evolution of fruiting bodies and related structures such as the cap, protective sheaths and spore-bearing structures (hymenophores). We further wanted to disentangle the genes which play a role in the formation of the fruiting body and its main tissue types. Therefore, we set up an experiment using a low-input RNA-seq method coupled with laser-capture microdissection and examined the transcriptome of seven stages and nine tissue types through the early development of the gilled model mushroom, Coprinopsis cinerea. More specifically, we attempted to answer the following questions:

1. What was the tempo of the speciation and extinction of species through the evolution of mushroom-forming fungi?

2. Was there any rapid rate shift regarding speciation or extinction rates indicating key innovation or mass extinction events, respectively?

3. If yes, could it relate to morphological traits?

4. Could morphological traits increase the diversification rate of species? We asked this question regarding six traits: cap production, the increased surface area of hymenophore, enclosed development, presence of protecting sheaths like universal veil or partial veil.

5. What genes are expressed during the transition from vegetative mycelium to primary and secondary hyphal knot developmental stages of Coprinopsis cinerea?

6. Could hyphal growth pattern changes be recognized at the transcriptome level of fruiting body development?

7. Are defense and surface coating associated genes more characteristic for cap, universal and partial veil than for other tissues?

8. What are the tissue-enhanced genes and when did them appear during evolution? 


\section{Materials and Methods}

\subsection{Comparative phylogenetic methods}

\subsubsection{Phylogenetic inference}

\subsubsection{Molecular data}

The molecular data served as the basis of the phylogenetic analysis of Agaricomycetes were generated and compiled by the members of the Fungal Genomic and Evolution lab and colleagues of the Joint Genomic Institute (JGI). Briefly, the nuclear large ribosomal subunit (nrLSU) locus was sequenced for 1,222 species, and additional sequences of this locus, translation elongation factor $1-\alpha(t e f 1-\alpha)$ and the RNA polymerase II second largest subunit ( $r p b 2$ ) were downloaded from public databases. Overall, 5,284 species have been carefully and evenly sampled from the Agaricomycetes, Dacrymycetes and Tremellomycetes while attempting to obtain specimens from undersampled geographical areas. The dataset for the 5,284 species consisted of 4,835 nrLSU, 1,252 rpb2 and 721 tefl- $\alpha$ sequences.

\subsubsection{Phylogenetic analysis}

Multiple alignments of each locus were generated by the members of the Fungal Genomic and Evolution lab using the Probabilistic Alignment Kit (PRANK, (Loytynoja and Goldman, 2005). Next, we manually corrected the multiple alignments, which was an essential and work-demanding step to correct homologous regions that were erroneously aligned by PRANK. The three individual alignments of the loci were concatenated into a super-alignment, which served as the data for inferring ML trees using the parallel version of RAxML v.8.1.2 (Stamatakis, 2014). Generalized time reversible (GTR) substitution model with gamma-distributed rate heterogeneity was used for three different partitions corresponding with the three loci. At this point, we used a phylogenomic tree (produced in the lab using 650 genes of 104 genomes) to infer a robust phylogeny and reconstruct deep nodes with confidence. In the ML analyses, we constrained a "backbone" topology by using the phylogenomic tree, supposing that genomic data could provide more robust information on deep nodes. After $245 \mathrm{ML}$ inferences were performed, we tested whether this number of trees cover a plausible set 
of topologies given the data. To do that pairwise Robinson-Foulds (RF) distances were calculated for each pair of trees using R package 'phangorn' v.2.0.2 (Schliep, 2011). Then, the rolling average, minimum and maximum were plotted as a function of the number of trees. Saturation of these statistics would suggest that phylogenetic uncertainty was adequately taken into account.

\subsubsection{Time calibration of phylogenetic trees}

Time calibration of phylogenetic trees with thousands of species is a computationally demanding task. Therefore, a two-step time calibration strategy was applied on ten trees that were selected from the $245 \mathrm{ML}$ trees using stratified random sampling. First, trees were hierarchically classified based on RF distances using the hclust function with Ward's clustering method in $\mathrm{R}$. Then, the resulting dendrogram was cut at the height which resulted in ten groups of trees. From each group, one tree was randomly selected. This stratified random sampling ensured to select trees randomly, but at the same time covering the entire topological diversity of the $245 \mathrm{ML}$ trees. The two-step time calibration strategy was based on using a robust and precise method (PhyloBayes v.4.1b, Lartillot et al., 2009) on a subset of species to infer parameters. The time calibration of trees with all species was performed using a less precise but a fast algorithm (FastDate developmental version, Akerborg et al., 2008) to which the information inferred in the first step was inputted. PhyloBayes analyses were run using a $10 \%$ subsampled dataset, which was obtained by randomly deleting $90 \%$ of the 5,284 species. A birth-death prior on divergence times, an uncorrelated gamma multiplier relaxed clock model, and a CATPoisson substitution model with a gamma distribution on the rate across sites were set in the analysis. A uniformly distributed prior was applied to the nine calibration points (for a detailed description of calibration points, see below). The convergence of chains (typically 15,000 cycles) was assessed by visually inspecting the likelihood value and the height parameter of the trees. We sampled every tree from the posterior, but to apply burn-in, the first 7,000 samples were discarded. The posterior estimates were summarized using the readdiv function of PhyloBayes.

FastDate implements a speed dating algorithm; therefore, it is ideal for performing time calibration on trees with thousands of species. Accordingly, FastDate was run on the complete species set, but the node ages constrained to the values of the $95 \%$ highest posterior densities of the ages inferred by PhyloBayes. FastDate analyses were run with 
time discretized into 1,000 intervals, and the ratio of sampled extant individuals set to 0.14 .

We used eight fossil calibration points and one constraint on the root (Table 1.). Only those fossils were included in the analyses, which could be matched to an extant clade with high confidence. We performed a fossil cross-validation procedure to check if there is any contradiction or conflict between the information that the different fossil calibration points hold. Fossil cross-validation was based on the work of Near et al. (Near and Sanderson, 2004). They argued that the effects of a calibration point on the inferred ages should not significantly influence the age of the nodes of other calibration points.

Table 1. The default calibration scheme using nine calibration points, including the root.

\begin{tabular}{|c|c|c|c|}
\hline Fossil & Calibration point & $\begin{array}{c}\text { Uniform time } \\
\text { prior boundaries }\end{array}$ & Reference \\
\hline $\begin{array}{c}\text { Quatsinoporites } \\
\text { cranhamii }\end{array}$ & Hymenochaetales & $127-250 \mathrm{Ma}$ & Smith et al., 2004 \\
\hline $\begin{array}{c}\text { Archaeomarasmius } \\
\text { leggettii }\end{array}$ & marasmioid clade & $92-180 \mathrm{Ma}$ & Hibbett et al., 1997 \\
\hline $\begin{array}{c}\text { Palaeoagaricites } \\
\text { antiquus }\end{array}$ & Agaricales & $105-210 \mathrm{Ma}$ & $\begin{array}{c}\text { G. O. Poinar \& } \\
\text { Buckley, 2007 }\end{array}$ \\
\hline Nidula baltica & Nidulariaceae & $45-90 \mathrm{Ma}$ & G. Poinar, 2014 \\
\hline Suilloid ECM & Suillaceae & $50-100 \mathrm{Ma}$ & Lepage et al., 1997 \\
\hline $\begin{array}{c}\text { Fungal termite combs } \\
\text { Trametites eocenicus }\end{array}$ & Trametes & $7-30 \mathrm{Ma}$ & $\begin{array}{c}\text { Duringer et al., } \\
2006\end{array}$ \\
\hline & & & Knobloch \& \\
& Root & $300-600 \mathrm{Ma}$ & \\
\hline
\end{tabular}

We carried out the fossil cross-validation analysis on one of the $10 \%$ subsampled trees using PhyloBayes with the settings described above. We performed eight molecular clock dating analyses using only one fossil at a time and calculated the sum of the square differences between molecular age estimates and fossil ages:

$$
S S_{x}=\sum_{i \neq x} D_{i}^{2}
$$


where $\mathrm{x}$ is the fossil calibration point used and $\mathrm{D}_{\mathrm{i}}$ is the difference between molecular age estimate and fossil age for fossil i. We ordered the SS values of each of the eight analyses in descending order and calculated the average squared deviation for all fossil calibrations:

$$
s=\frac{\sum_{x=1}^{n} \sum_{i \neq x} D_{i}^{2}}{n(n-1)}
$$

Next, the analysis with the highest SS value was removed and s was recalculated. We continued this process until only two analyses remained. In parallel, we checked whether the variance of $s$ had significantly been changed after removal of a fossil by performing one-tailed F-tests $(\mathrm{p}<0.05)$.

\subsubsection{Genome-based molecular dating}

We performed our genome-based molecular dating on the 650-gene and 104species phylogenomic dataset, which was used as a backbone tree in the 5,284 speciestree inference. First, we subsampled genes, so the first 70 most conserved genes were selected by calculating the mean genetic distances for each gene using the dist.alignment function of the seqinR R package v.3.4-5 (Charif and Lobry, 2007). Second, we added three species (Cyathus striatus, Pycnoporus cinnabarinus, Suillus brevipes) to the dataset to enable more accurate placement of fossil calibration points. We further excluded two taxa that harbored ambiguous positions. Homologous sequences in the additional genomes were searched using blastp v.2.7.1 (Camacho et al., 2009) with one randomly selected gene from each of the 70 gene families as a query. We considered the blast hit with the smallest E-value as one-to-one orthologs if the second-best hit had a significantly worse E-value (by 20 orders of magnitude). Multiple alignments of protein clusters were produced using PRANK v.100802 (Loytynoja and Goldman, 2005) with default settings. Conserved blocks of the multiple alignments were selected using Gblocks v.0.91b (Talavera and Castresana, 2007) by setting the minimum length of a block to 5 and by allowing gap positions in half of the sequences. A phylogenomic tree was constructed by RAxML v.8.2.11 (Stamatakis, 2014) under the WAG + G substitution model partitioned by genes.

We used the momctree method implemented in PAML v.4.8a (Yang, 2007) to time calibrate the phylogenomic tree. The independent-rates clock model, WAG substitution model, and approximate likelihood calculation (Thorne et al., 1998) were 
applied. The birth rate, the death rate, and the sampling fraction of the birth-death process were set to 1,1 and 0.14 , respectively. Both the shape and the concentration parameter of the gamma-Dirichlet prior for the drift rate coefficient $(\sigma 2)$ were set to 1 while the scale parameter was set to 100 . To find the best parameters of the gamma-Dirichlet prior for the overall rate, the substitution rates of each gene were estimated by codeml function under a global clock model. By calculating the mean substitution rate of all loci separately and examining the density plot of the rates, we set up a gamma-Dirichlet prior that was reasonably fit to the data and the shape, the scale, and the concentration parameter were set to 5, 90.74 and 1, respectively. This setting resulted in an average substitution rate per site per time unit of 0.055 . We set the time unit to $100 \mathrm{Ma}$ and applied uniform priors on calibrations points with lower and upper hard bounds. MCMC analysis was run for 80,000 iterations with sampling every $30^{\text {th }}$ tree from the posterior. After three independent analyses were run, the first 20,000 iterations were discarded as a burn-in, and the convergence of log-likelihood values was visually inspected.

3.1.1.5. Validating and comparing inferred ages with previous molecular clock estimates

Previous studies inferred different ages estimates than we did in this study (Floudas et al., 2012; Kohler et al., 2015), thus we wanted to test what lies behind the differences. Therefore, we performed additional molecular clock analyses on genomic data using r8s v.1.81 (Sanderson, 2002). First, we ran a series of molecular clock analyses to determine the optimal smoothing parameter $(\lambda)$ by performing a cross-validation analysis. We tested seven smoothing parameters from $10^{-3}$ to $10^{3}$ with one order of magnitude increments. The additive penalty function was applied and the optimization was run 25 times, starting from independent starting points. In one optimization step, after reaching an initial solution, the solution was perturbed and the truncated Newton optimization was rerun 20 times. Using the optimal smoothing parameter, we performed three molecular clock analyses. First, we used the same fossil calibration scheme as for the 5,284-species phylogenetic dataset ('default calibration scheme'). Second, we replicated the analyses of Kohler et al. (Kohler et al., 2015) on our tree, applying their fossil calibration points ('Kohler et al. calibration scheme 1 '). In this, the suilloid ectomycorrhiza and Archaeomarasmius leggettii fossils were respectively placed in the split of Suillinae/Paxillinae/Sclerodermatinae and in the most recent common ancestor (MRCA) of Gymnopus luxurians and Schizophyllum commune with a uniform time prior 
of 40-60 Ma and 70-110 Ma. Third, we used the calibration points applied by Kohler et al., but we placed the two fossils (suilloid ectomycorrhiza and Archaeomarasmius leggettii) in the MRCAs (crown node) of the Suillaceae and marasmioid clade, respectively ('Kohler et al. calibration scheme 2'). The third analysis was important, because some essential genomes were not available for Kohler et al., and they could only place fossils on the stem nodes of clades. In all analyses, we constrained the age of the root to be between $300 \mathrm{Ma}$ and $600 \mathrm{Ma}$ ago.

\subsubsection{Accounting for non-random and incomplete taxon sampling}

One of the assumptions of most diversification analyses is that the inferred tree consists of all existing species from the taxon examined. However, this assumption does not hold in most cases (Fitzjohn et al., 2009); therefore, corrections on the species number were applied in our analyses. First, we screened all orders of the Agaricomycetes, Dacrymycetes and Tremellomycetes in Species Fungorum (CABI, 2018) and gathered all species with a custom java program (the code is available upon request). We took into account taxonomic and nomenclatural synonymy through the automated screening as Species Fungorum indicated it. Based on the information retrieved from Species Fungorum, we could assign a sampling fraction to each genus in our phylogeny, creating a genus-specific sampling fraction database.

In further analyses, two basic sampling correction strategies were applied. Using the genus-specific sampling fraction data, we applied the built-in correction procedure of the given program. On the other hand, we produced a 'skeletal tree' (sensu Fitzjohn et al., 2009), where species were both evenly and randomly sampled from every genus. To create a 'skeletal tree' we iteratively deleted species from genera that were oversampled, until sampling fractions of each genus corresponded their known size based on Species Fungorum. To do this, we performed a hypergeometric test at each iteration of species removal $(\mathrm{P}<0.05)$ and the elimination of species was stopped when oversampling disappeared.

\subsubsection{Trait independent diversification analyses}

\subsubsection{Bayesian analysis of macro-evolutionary mixtures (BAMM)}

To infer species diversification rate through the evolution of mushroom-forming fungi, we used BAMM v.2.5.0 (Rabosky, 2014). The BAMM algorithm can calculate rate 
heterogeneity across lineages and through time, while it detects shifts in the diversification rate. We analyzed ten chronograms and ran MCMC analyses for 100 million generations using four independent chains per analysis with 50 million generations as burn-in. Prior parameters were optimized using the setBAMMpriors function in BAMMtools v.2.1.6 (Rabosky et al., 2014), except for the prior on the expected number of shifts, which was set to 270 based on preliminary runs. We accounted for incomplete taxon sampling, as described above. We checked the convergence of chains by visually inspecting the convergence of likelihoods and by calculating the effective sample size (ESS) and the Geweke's diagnostic (Geweke, 1992) of loglikelihoods, numbers of shifts and evolutionary rate parameters using functions in CODA 0.19-1 (Plummer et al., 2006). To ensure that a shift is highly supported by the data and the prior had a negligible contribution to it, we examined only shifts with a prior-toposterior marginal odds ratio exceeding 5 to which we refer as core shifts (Rabosky et al., 2014). To visually depicting tree-wide evolutionary patterns we plotted mean evolutionary rates (speciation, extinction and diversification rates) through time using plotRateThroughTime and getRateThroughTimeMatrix functions of the BAMMtools package. Lineage through time (LTT) plots were created by using the ltt.plot function in the R package ape v4.1. (Paradis et al., 2004).

\subsubsection{Detecting mass extinction events}

We performed analyses using the compound Poisson process on mass extinction times (CoMET) model (Höhna, 2015; May et al., 2016) to test the occurrence of mass extinctions through the evolution of mushroom-forming fungi. First, we conducted a model comparison on ten chronograms using the CoMET model with a constant-rate birth-death process implemented in the TESS 2.1.0. R package (Höhna, 2013). We compared models with and without mass extinction events based on marginal likelihoods. We allowed the occurrence of a mass extinction event along the entire time span of a tree and we set the survival probability of species to 0.1 (corresponding to $10 \%$ of the species surviving a mass extinction event). The overall sampling fraction of species was set to 0.14. MCMC analyses were run for 20,000 generations and the first 2,000 posterior samples were discarded as a burn-in. Marginal likelihoods were estimated using stepping stone simulation using 100 stepping stones per analysis. 
Then, we performed reversible jump Markov chain Monte Carlo (rjMCMC) analyses under the CoMET model to sample from the space of episodically varying birthdeath processes with mass extinction events. In this analysis, we also tested the significance of the occurrence of a mass extinction event by performing model tests within time intervals. Log Bayes factor values higher than six $(\operatorname{lnBF}>6)$ were considered strong support for a model, following Höhna et al. (Höhna et al., 2015) and Kass and Raftery (Kass and Raftery, 1995). We examined the sensitivity of the posterior probabilities to different prior settings in preliminary analyses of a randomly chosen tree. We examined models with two or ten expected mass extinction events, with 30, 100 or 270 expected rate changes and with survival probabilities of $0.001,0.05$ or 0.3 . All these preliminary analyses were run for 1.6 million generations with 100,000 generations as burn-in. We used a log-normal prior on speciation and extinction rates with a mean of 0.2 and 0.15 and a standard deviation of 0.5 and 0.5 , respectively. In the final analyses, we used all ten chronograms inferred by using our two-step time calibration strategy (see, paragraph 2.1.1.3) and an empirical hyper-prior on rate parameters based on 200,000 iterations with 100,000 burn-in. The priors on the number of expected mass extinction and the expected rate changes were set to 2 and 30, respectively, based on the results of preliminary analyses. We set the survival probability to 0.05 . Analyses were run for 3 million generations with 1 million generations as burn-in. The convergence of the analyses was checked by visually inspecting the log-likelihood values and by computing the effective sample size and the Geweke diagnostic for the log-likelihoods, the number of speciation rate shifts, the number of extinction rate shifts and the number of mass extinction events using the effectiveSize and the geweke.diag functions of the CODA 0.19.-1 package (Plummer et al., 2006), respectively. To check the convergence of interval-specific parameters, we used the tess.plot.singlechain.diagnostics function of the TESS package.

\subsubsection{Macro-evolutionary analyses of morphological traits}

\subsubsection{Character coding}

We included six discrete morphological traits into our macro-evolutionary analyses: (1) fruiting body types, (2) presence or absence of the cap, (3) enclosed development, (4) presence of universal veil, (5) presence of partial veil and (6) increased hymenophore surface area (Table 2., Figure 3., for detailed description see below). We 
highly relied on literature data through all trait codings. First of all, we summarized 52 number of histological studies from the last $>100$ years, which could serve detailed and thorough information on the most common 94 genera (Electronic Appendix 1). If a species had plectological information we made phylogenetically informed extrapolations to the whole genus except for species with a unique morphology. In addition to the plectological information, we gathered data from taxonomical publications, species descriptions. In rare cases, we visually inspected images of fruiting bodies in various developmental stages.

Table 2. The six characters and their character states were examined in this study.

Character

\begin{tabular}{ll}
\hline Fruiting body types & $\begin{array}{l}\text { (1) No fruiting body, (2) resupinate, (3) pileate, (4) cyphelloid, (5) } \\
\text { gasteroid/secotioid, (6) coralloid/clavarioid }\end{array}$ \\
\hline Cap & (1) Absence of cap, (2) Presence of cap \\
\hline Enclosed development & $\begin{array}{l}\text { (1) Open development, (2) Semi-enclosed development, (3) Enclosed } \\
\text { development }\end{array}$ \\
\hline Universal veil & (1) Absence of universal veil, (2) Presence of universal veil \\
\hline Partial veil & (1) Absence of partial veil, (2) Presence of partial veil \\
\hline $\begin{array}{l}\text { Increased hymenophore } \\
\text { surface area }\end{array}$ & $\begin{array}{l}\text { (1) Smooth hymenophore, (2) weakly structured hymenophore, (3) well- } \\
\text { developed hymenophore }\end{array}$ \\
\hline
\end{tabular}

We characterized six different states of fruiting body types (Table 2., Figure 3.). Resupinate fruiting bodies were defined as crust- or sheet-like morphologies that attached or intergrown to the substrate without considering the thickness of the fruiting body or the type of the hymenophore. The pileate type was defined as having a distinguishable cap with a positive gravitropic hymenophore. We followed the definition of Bodensteiner (Bodensteiner et al., 2004) to code cyphelloid species, that is cup- to barrel-shaped minute $(<1-2 \mathrm{~cm})$ fruiting bodies with smooth hymenophore covering a concave inner surface. The gasteroid types included species that produce spores internally. Therefore, no distinction was made between puffball, secotioid, sequestrate and false-truffle morphologies, and all were coded as one category. Coralloid and clavarioid fruiting bodies were defined as having club-shaped or branched, erect morphology, but no differentiated cap is developed.

To determine which species produce a cap was generally straightforward based on literature data. However, in the few cases where the absence (state 0) or the presence 
(state 1) was not prominent, e.g., rudimentary or reduced cap, species coded as uncertain (state $0 / 1)$.

In the case of enclosed development, we defined three character states (Table 2., Figure 3.). The character state assignment was based on whether the developing hymenophore is open to the environment or insulated from it at some point during development. Open development (state 0) refers to fruiting bodies with hymenophore exposed to the environment from the beginning to the end of development. This state corresponds with the definitions gymnocarpy sensu Reijnders (Reijnders, 1948; Moore et al., 1984) and exocarpy without any metablemas sensu Clemencon (Clémencon, 2012). Semi-enclosed development (state 1) was defined as fruiting-bodies with a faint tissue covering the hymenophore at the early developmental stages but later, it is disrupted or vanished. In the case of enclosed development (state 2), a tenacious tissue is present and covers the hymenophore or the whole fruiting body from the beginning of the development until spore maturation and release. By definition, we included gasteroid and secotioid mushrooms in the character state 2 because spore production entirely takes place in the protection of fungal tissues. The semi-enclosed development corresponds to the definitions of hypovelangiocarpy sensu Reijnders, while the enclosed development fits the descriptions of angiocarpy sensu Reijnders (Reijnders, 1948; Moore et al., 1984) or endocarpy and nodulocarpy sensu Clémencon (Clémencon, 2012).

To further disentangle the effect of the protection of different tissue layers, we also examined the universal veil (absence: state 0, presence: state 1) and the partial veil (absence: state 0, presence: state 1) in separate analyses (Table 2., Figure 3.). Universal veil envelopes the whole fruiting body and leaves tissue remnants on the base of the stipe and the surface of the cap in mature fruiting bodies. Contrarily, the partial veil spans between the edge of the cap and the top of the stipe, covering only the developing hymenophore. The partial veil has tissue remnants only on the upper part of the stipe in mature fruiting bodies. During the character coding of these traits, we relied on the plectological studies and taxonomical descriptions mentioned above.

Producing more spores per mass of the fruiting body can increase the reproductive success of the organisms. Therefore, we included in our macro-evolutionary analyses a trait associated with the size of the hymenophore surface area (Table 2., Figure 3.). 
A) Fruiting body types

Resupinate

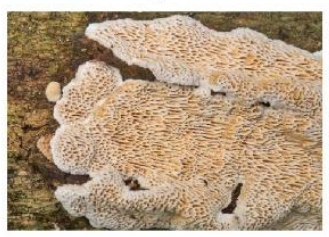

Coralloid/Clavarioid

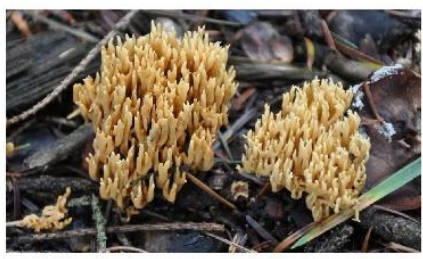

Gasteroid

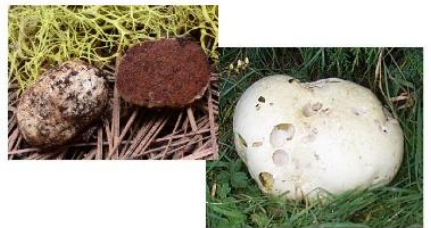

Cyphelloid

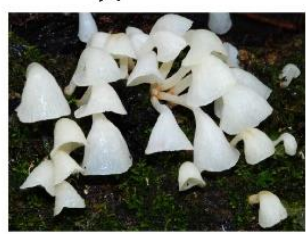

Pileate

\section{B) Cap} Presence/Absence

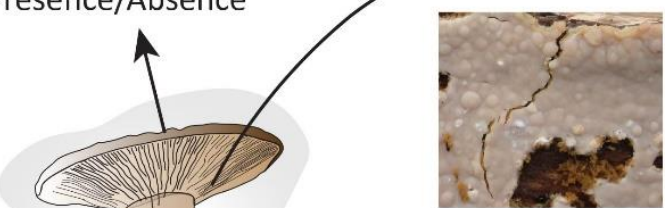

Smooth

hymenophore

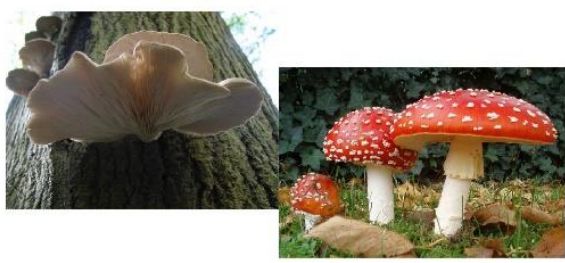

C) Hymenophore

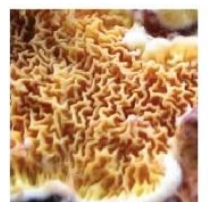

Weakly structured hymenophore

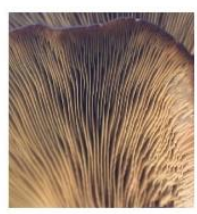

Well-developed hymenophore

F) Enclosed development

D) Universal veil Presence/Absence

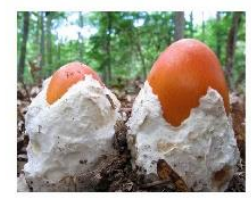

E) Partial veil Presence/Absence

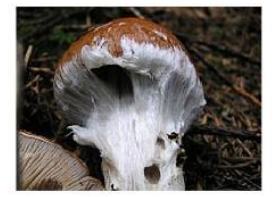

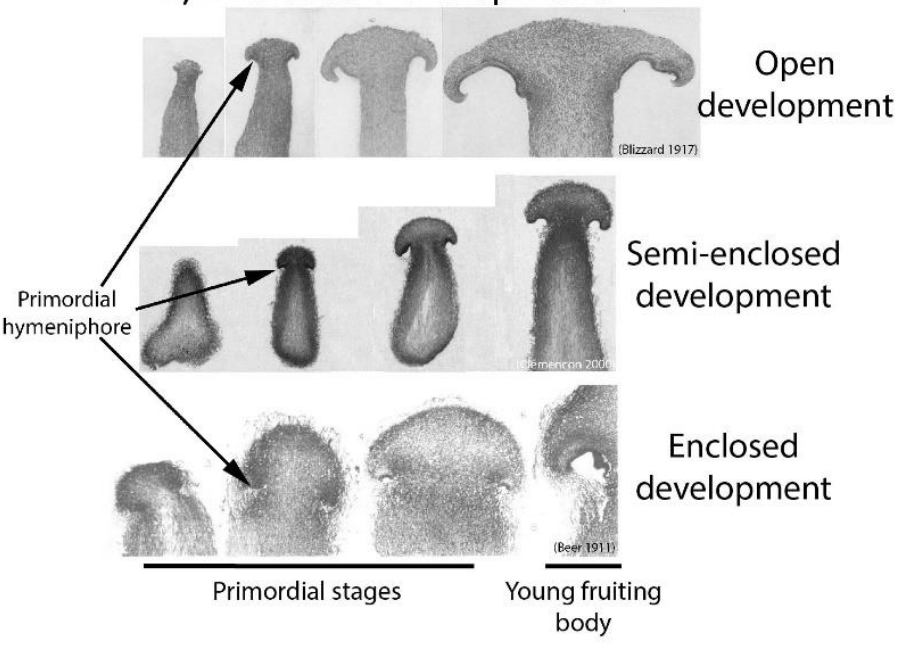

Figure 3. (Previous page). The morphological traits which were examined through the macroevolutionary analyses. A) Main fruiting body types. B-F) Morphological traits of mushrooms which could give evolutionary advantage for species. We gave a schematic figure of a pileatestipitate mushroom to depict the main morphological traits of a complex fruiting body. B) Cap trait contains presencelabsence data. C) The three states of the increased hymenophore surface area traits. $D$ ) and $E$ ) show the general appearance of the universal veil and partial veil on mature fruiting bodies. F) The enclosed development trait. The developing hymenophore is open to the environment from the earliest primordial stages (open development) or is closed either in the earliest primordial stage (semi-enclosed development) or through to the young fruiting body stage (enclosed development). Photo courtesy: cdn-0.enacademic.com; biolib.cz; first-nature.com; lh3.googleusercontent.com; upload.wikimedia.org; miro.medium.com 
Smooth hymenophore (state 0) was assigned to those species where the sporebearing structure spanned on a plain and smooth surface. Weakly structured hymenophore (state 1) represented hymenophore with small structures such as veins, ridges or bumps, which barely increase the surface of the hymenophore. The most advanced hymenophores were termed as well-developed hymenophores (state 2), including complex and pronounced structures like gills pores and teeth. Through all character coding procedures, we handled those cases as uncertain states where a state was ambiguous or was hard to classify, e.g., hymenophore with moderately developed veins.

\subsubsection{Character state evolution}

We modeled character evolution using the Mk model implemented in BayesTraits 2.0 Linux 64 Quad Precision alternative build (Pagel and Meade, 2007) and the BiSSE (Maddison et al., 2007) or MuSSE (Fitzjohn, 2012) models implemented in the diversitree package v.0.9-10 (Fitzjohn, 2012). BayesTraits analyses (maximum likelihood and MCMC) were performed on $245 \mathrm{ML}$ trees (see paragraph 2.1.1.2.) using the MultiState module of the program. Before the final MCMC analyses, we tried several prior distributions (uniform, exponential, gamma and the hyper-prior versions of these) with different settings. Based on preliminary analyses, we found that the gamma distribution was the most optimal, therefore in further analyses we used a gamma hyper-prior with different prior distributions for each parameter (Electronic Appendix 2). All preliminary BayesTraits analyses were conducted with the following settings: 1,010,000 generations, 10,000 generations as burn-in and sampling every 500th generation. We forced Markov chains to spend 200,000 generations on each tree using the equaltree option, with 100,000 generations as burn-in and sampling every 500th generation. The marginal likelihood was estimated by the stepping stone method (Xie et al., 2011) using 50 stones with a chain length of 5,000. All analyses in BayesTraits were repeated three times to check the congruence of independent runs. Analyses in diversitree were performed as described in the next section. For every character (Table 2.), we tested the importance of all character state transition rates by constraining them to 0 in individual analyses and evaluate the likelihood ratio and the Bayes Factors. We also tested whether uneven rates among transition rate pairs (e.g., q01 and q10) were supported. To do this, we constrained the transition rate pairs to be equal and compared the full model with the constrained model using likelihood ratio tests and Bayes factor. Each of the constrained models was compared to the best fit model based on log-likelihood values (ML analyses in 
BayesTraits), log-Bayes factors (MCMC analyses in BayesTraits) or likelihood ratio test (LRT) (ML analyses of BiSSE and MuSSE models in diversitree). In BayesTraits, a difference of 4.00 log-likelihood units (maximum-likelihood approach) or a difference of 10 log marginal likelihood units (MCMC approach) was considered as significant support for a model over another, while in the BiSSE and MuSSE analyses significance was assessed using likelihood ratio tests (LRT, P < 0.05)

\subsubsection{Trait dependent diversification analysis}

We used ten chronograms to analyze trait-dependent diversification under the BiSSE and MuSSE models implemented in the R package diversitree v.0.9-10 (Fitzjohn, 2012). The maximum likelihood search started from the point in the parameter space determined by the function starting.point.bisse. Bayesian MCMC was performed using an exponential prior, defined as $1 /(2 r)$, where $r$ is the character independent diversification rate. We applied the built-in function for correcting incomplete taxon sampling using the state-specific sampling fractions retrieved from Species Fungorum (see above). We first optimized the MCMC sampler's step size argument by running 100 generations, and then we ran MCMC analyses for 20,000 generations with burn-in set to $10 \%$. The convergence of the chains was visually checked based on likelihood values and parameter estimates. We performed likelihood ratio tests $(p<0.05)$ to evaluate the significance of the differences between the speciation or extinction rates of lineages with different character states.

\subsubsection{Creating states-through time plot}

To have an overview of the macro-evolution of main fruiting body types (Table 2.), we reconstructed ancestral states using stochastic character mapping as implemented in phytools v.06-20 (Revell, 2012). This method is a modified version of a previously published algorithm (Huelsenbeck et al., 2003), which samples discrete character histories from the posterior probability distribution. We performed the analysis with the make.simmap function on ten time-calibrated trees under a Markov model with all rates different. The stochastic character histories were simulated 5,000 times. We plotted state posterior probabilities through time using a custom $\mathrm{R}$ script (available at github.com/vtorda/StatesThroughTimePlot). Briefly, we summarized character-state posterior probabilities through the time scale of our ten chronograms split into 100 bins. 
The plot was created using ggplot and geom_area functions of ggplot2 v.2.2.1 package (Wickham, 2009).

\subsection{Laser-capture microdissection coupled low-input RNA sequencing}

\subsubsection{Exploring tissue differentiation of Coprinopsis cinerea}

Coprinopsis cinerea strain A43mutB43mut was grown on a YMG-Trp media (0.4 $\mathrm{m} / \mathrm{m} \%$ yeast extract, $1 \mathrm{~m} / \mathrm{m} \%$ maltose, $0.4 \mathrm{~m} / \mathrm{m} \%$ glucose, $0.01 \mathrm{~m} / \mathrm{m} \%$ DL Tryptophan, $1 \mathrm{~m} / \mathrm{m} \%$ agar) for 5 days at $37^{\circ} \mathrm{C}$ in dark and then at $25^{\circ} \mathrm{C}$ in 12 -hour-dark/12-hour-light regime. During primordium sampling, we paid attention to pick different sized developmental stages gradually. We gathered hyphal knot stages only in the first few days, and we only sampled primordia bigger than $1.5-2 \mathrm{~mm}$ after 4-5 days. These growing and sampling conditions were used in both preliminary and low-input RNA-seq examinations as well. We strongly built on general (Carson, 1997) and specialized (Espina et al., 2006; Thakare et al., 2014) histological methods during the development of our protocol (Appendix 1.). Through preliminary histological analyses, we used halfstrength Karnovsky's fixative (1\% paraformaldehyde, $2 \%$ glutaraldehyde in phosphatebuffered saline (PBS)) and did not implement precautions related to the preservation of RNA integrity and quality. Through the preliminary analyses, tissue sections were examined in water or stained with a solution of $1 \%$ methylene blue, $1 \%$ borax and $1 \%$ Azure II (MBA, Matthews \& Niederpruem, 1973).

We also investigated the dependence of the different types of primordia from the time of day. Sampling started from the third day of the dark/light period. For three days, we embedded five materials for cryo-sectioning at two-two time points during the day and the night regimes. By thoroughly examining numerous histological sections, we wanted to screen and describe the early developmental stages of $C$. cinerea and draw parallels between our histological descriptions and literature data.

\subsubsection{Optimizing a tissue-specific low-input RNA-seq workflow}

Several adjustments and optimizations were performed on existing methods to achieve high-quality RNA extractions. It is crucial to preserve both tissue and RNA 
integrity as much as possible through laser-capture microdissection coupled with lowinput RNA sequencing. Achieving this can be conflicting, because, for example, fixatives, which can well-preserve tissue integrity, also cross-bind molecules (including RNA). Moreover, many tissue embedding methods require a long incubation time, which can negatively affect RNA quality. Therefore, we attempted to find a compromise between tissue and RNA preserving methods at every step through the whole protocol.

\subsubsection{Fixation}

Farmer's solution was used as a fixative, which contains $75 \%$ absolute ethyl alcohol and 25\% glacial acetic acid, causing no cross-binding of RNA molecules as glutaraldehyde containing fixatives (e.g., Karnovsky fixative) would do. Five minutes long -0.8 bar vacuum incubation was applied three times to remove air from intercellular regions helping the fixative penetrate the sample thoroughly. Hyphal knots and primordia were sampled with a small layer of agar at the bottom $(\sim 5 \times 5 \mathrm{~mm}$ and $1 \mathrm{~mm}$ thick), which allowed us to handle samples through this agar sheet, protecting samples from any physical impact. Vegetative mycelium was sampled with a tip of a needle, forming a more or less dense mycelium ball, which was further handled as any other tissue samples.

\subsubsection{Sample preparation for cryo-sectioning}

We used a cryo-microtome (Leica CM1860 UV) to prepare histological sections for LCM, because keeping samples under cold conditions lowers the activity of RNases. Before cryo-sectioning, tissue samples were infiltrated with a $30 \mathrm{~m} / \mathrm{m} \%$ sucrose solution (in 1x PBS) using a series of concentrations (Table 3.). Sucrose can serve as a cryoprotective agent and can help to achieve a better quality of histological sections. Every step in Table 3. took 15 minutes, and a rotator (Biosan Multi Bio RS-24) was used to facilitate infiltration. Samples were embedded into a cryomount (Histolab OCT Cryomount) after the infiltration step and were frozen using dry ice. Frozen samples were stored at $-70^{\circ} \mathrm{C}$ for further examination. 
Table 3. Series of sucrose solutions used to infiltrate samples with a $30 \mathrm{~m} / \mathrm{m} \%$ sucrose solution.

\begin{tabular}{|l|c|c|}
\hline & $\begin{array}{c}10 \mathrm{~m} / \mathrm{m} \% \text { sucrose } \\
\text { solution }\end{array}$ & $\begin{array}{c}30 \mathrm{~m} / \mathrm{m} \% \text { sucrose } \\
\text { solution }\end{array}$ \\
\hline & \multicolumn{2}{|c|}{ Ratio of the solutions } \\
\hline Step 1 & 1 & 0 \\
\hline Step 2 & 2 & 1 \\
\hline Step 3 & 1 & 1 \\
\hline Step 4 & 1 & 2 \\
\hline Step 5 & 0 & 1 \\
\hline Step 6 & 0 & 1 \\
\hline Step 7 & 0 & 1 \\
\hline
\end{tabular}

At this step, the performance of two RNA protecting agents have also been tested: QIAGEN RNAlater RNA stabilization reagent and 2-mercaptoethanol. Fixation, infiltration and embedding were applied on stage 3 primordium, but with different treatments: (1) no treatment, (2) putting $10 \mu \mathrm{l} / \mathrm{ml}$ QIAGEN RNAlater RNA stabilization reagent or (3) putting $20 \mu \mathrm{l} / \mathrm{ml}$ 2-mercaptoethanol into the sucrose solutions and (4) positive control where RNA was extracted from a fresh stage 2 primordium. We extracted RNA from samples after sucrose infiltration using Quick-RNA Microprep Kit (Zymo Research) following the manufacturer's instructions. RNA concentration was measured using NanoDrop 2000 spectrophotometer (Thermo Scientific ${ }^{\mathrm{TM}}$ ) and the four RNA extracts were inspected using agarose gel electrophoresis.

\subsubsection{Cryo-sectioning}

Cryo-sectioning parameters were adjusted to obtain the best histological sections. We tried a series of cutting temperatures between $-25{ }^{\circ} \mathrm{C}$ and $-15{ }^{\circ} \mathrm{C}$, different section thicknesses between 5-20 $\mu \mathrm{m}$ and tested three types of slides: (1) glass slide, (2) PEN (polyethylene naphthalate) membrane covered glass slide (Carl Zeiss ${ }^{\mathrm{TM}}$ ) and (3) PEN membrane glass slide additionally covered with poly-L-lysine. Membrane slides were covered with poly-L-lysine in house. First, membrane slides were exposed to ultraviolet light for at least 1 hour to improve adhesion. Then $1.5 \mathrm{ml} 0.01 \%(\mathrm{w} / \mathrm{v})$ poly-L-lysine solution (Sigma-Aldrich) was pipetted on the surface of the membrane and incubated at room temperature for 10 minutes. At the end of the incubation time, the poly-L-lysine solution was removed from the membrane and the slide dried on air. When the membrane was entirely dried, the poly-L-lysine treatment was applied again. Finally, a 3 hours long ultraviolet treatment was applied on the surface of the slide. Frozen tissue sections were mounted on pre-warmed slides $\left(\sim 30^{\circ} \mathrm{C}\right)$ to improve mounting. 


\subsubsection{Preparation for laser capture microdissection (LCM)}

Dry and completely water-free tissue sections can improve the performance of LCM. Therefore, a series of alcohol solutions were applied to remove water and the embedding material from the cryo-sections using staining jars. Slides with tissues attached to them were immersed in $75 \%$ ethyl alcohol for 30 seconds then moved into Diethyl pyrocarbonate (DEPC) treated nuclease-free water for 30 seconds. These two steps were repeated three times, which ensured to remove the embedding material thoroughly. Then, slides were immersed in $75 \%, 90 \%$, and finally absolute alcohol using 30 seconds long incubation time at each step.

\subsubsection{Laser capture microdissection}

LCM was performed using a Zeiss PALM MicroBeam microscope and the PALM RoboSoftware v.4.6. First, we screened the histological sections by using a 10x objective (Numerical aperture $=0.3 ; 100 \mathrm{x}$ magnification) to record the size dimensions of the primordia and mark the different tissue types based on the shape and the structure of different regions. The 'joint contour' was used in the RoboSoftware to select and mark tissue of interest, providing a narrow tissue $(5 \mu \mathrm{m})$ to be attached to the whole section. By using 'joint contour', the dissected tissues do not change position, or the tissue of interest can be protected by applying the catapult laser beam only on the joint tissue area. We attempted to hold at least a $5 \mu \mathrm{m}$ safety zone from the surrounding tissues to minimize the possibility of contamination. After all, tissues were marked by 'joint contour', laser dissection was performed with 60x objective (Numerical aperture $=0.75,600 \mathrm{x}$ magnification). Objectives with higher magnification gave a smaller diameter for the laser beam and thus destroyed a narrower part of the tissue. Laser pressure catapulting was performed using a 10x objective targeting the joint region of the dissected tissue. The cap of the $0.5 \mathrm{ml}$ microcentrifuge tubes (Eppendorf, DNA LoBind Tubes) prepared in advance was placed as close as possible above the tissues (roughly less than a millimeter space left). Laser dissected tissues were collected into the cap of tubes, which contained $20 \mu 1$ extraction buffer (PicoPure ${ }^{\mathrm{TM}}$ RNA Isolation Kit). The locked tubes were placed into liquid nitrogen until the end of the LCM procedure.

\subsubsection{Cell disruption and RNA extraction}

Only minute amounts of starting material ( 15 laser dissected tissue samples in 20 $\mu 1$ extraction buffer) were available for RNA extraction, therefore methods for fungal cell 
disruption were limited. Two types of cell disruption methods were tested: chemical and physical disruptions. Through chemical cell disruption, we tried four compounds using two enzyme solutions and two extraction buffers of different vendors. One of the enzyme solutions was a chitinase (Sigma-Aldrich, Chitinase from Streptomyces griseus, $1 \mathrm{mg} / \mathrm{ml}$ in DEPC treated potassium phosphate buffer $(\mathrm{pH}=6))$, and the other was an enzyme mixture $(1.5 \%$ snail enzyme extraction (prepared at the Department of Microbiology, University of Szeged), $0.5 \%$ Sigma-Aldrich chitinase in $\mathrm{KCl}-$ phosphate buffer $(\mathrm{pH}=$ 5.8)). The two extraction buffers of the PicoPure ${ }^{\mathrm{TM}}$ and MACHEREY-NAGEL ${ }^{\mathrm{TM}}$ NucleoSpin ${ }^{\mathrm{TM}}$ vendors were used.

First, we tested the cell wall digesting activity of the chitinase and the enzyme mixture. To do that, we digested fresh mycelium in a PCR tube containing $50 \mu 1$ chitinase solution for 10 and 30 minutes. Next, we tested the enzyme mixture, but here we used a $500 \mu 1$ volume. In both cases, we checked hyphal morphology under a bright field microscope. We also tested whether the chitinase and the enzyme mixture affected the RNA extraction. To do that, we mixed an RNA extract and a digesting solution in 1:1 or $1: 2$ volume ratio and incubated at $37^{\circ} \mathrm{C}$ for 30,90 and $180 \mathrm{~min}$. We examined the samples using agarose gel electrophoreses after the incubation time ended. We also used a heatshock cell disruption procedure similar to that used by Teichert and coworkers (Teichert et al., 2012), but we kept the samples in the cap of the tubes through the whole procedure and did not use vortexing (see details in Appendix 1).

RNA extraction was performed by using the PicoPure ${ }^{\mathrm{TM}}$ RNA isolation kit following the manufacturer's protocol. We checked the quality and the quantity of RNA extracts with a 2100 Bioanalyzer Instrument (Agilent Technologies). We considered the RNA integrity number (RIN) higher than 7.5 or RIN between 6 and 7.5 as excellent or sufficient quality for RNA sequencing, respectively. Prior to sending the RNA extracts to the LC Sciences Company for reverse transcription, cDNA amplification and sequencing, we added an external RNA control (ERCC RNA Spike-In Mix, Invitrogen ${ }^{\mathrm{TM}}$ ) into the samples. Spike-In controls ensured to detect if any bias happened through downstream procedures (Baker et al., 2005; Reid, 2005). Reverse transcription and cDNA amplification were done by using the SmartSeq2 protocol (Picelli et al., 2013, 2014) and paired-end (150 bp) sequencing was performed on an Illumina Hiseq 4000 instrument by LC Sciences LLC. 


\subsubsection{Quality check and analysis of RNA sequencing data}

3.2.3.1. Read processing and align reads to the reference genome

Read quality was checked using the FastQC v.0.11.8 (Andrews et al., 2018) and MultiQC v.1.7. (Ewels et al., 2016) programs. Quality trimming of reads was performed using the BBMap package (Bushnell, 2020) by setting the minimum read length threshold to 40 and the minimum quality score threshold to 25 . The Coprinopsis cinerea AmutBmut pab1-1 v1.0 reference genome (Muraguchi et al., 2015) was downloaded from the JGI Mycocosm portal (Grigoriev et al., 2014) in December 2019. The quality trimmed reads were mapped to the reference genome using the STAR v.020201 program (Dobin et al., 2013) with two-round mapping. In the first round, the results of every sample were summarized to identify junction sites. This junction site information data was used in the second round read mapping. STAR mapping was performed with the following settings: outFilterScoreMinOverLread: 0.4, outFilterMatchNminOverLread: 0.4, alignIntronMax: 3000, runThreadN: 30 . The aligned reads were counted for each gene in the genome using the summarizeOverlaps function in the GenomicAlignments v.1.22.1 package (Lawrence et al., 2013) using the "Union" mode. This method counted a read if it overlapped with any portion of an exonic region. However, reads were discarded if they overlapped with multiple genes.

\subsubsection{Library normalization}

We performed both the full quantile library size normalization (Bullard et al., 2010) and GC normalization procedures (Risso et al., 2011). The latter could be significant in our analysis because the SmartSeq2 protocol applies a cDNA amplification step where QC-rich cDNA templates could be favored by PCR (Polz and Cavanaugh, 1998). Before normalization procedures were performed using the EDAseq v.2.20.0 package (Risso et al., 2011), we filtered out those genes which possessed less than 10 counted reads across all samples.

\subsubsection{Expression analysis in DESeq2 framework}

DESeq2 v.1.26.0 package was used to perform differential expression (DE) analysis (Love et al., 2014). The expression values were calculated using a negative binomial (NB) generalized linear model (GLM), allowing to define GLM models with multiple conditions. One huge advantage of the DESeq2 framework is that one condition 
can be analyzed while the effect of others is controlled. We generally used three conditions in our models: batch effect, developmental stages and tissues. We considered one sequencing bundle as a unique batch, therefore the 'batch effect' variable contained four factor levels. Another advantage of GLM is the possibility of detecting outliers based on Cook's distance: a measure of the influence of a data point on the overall regression model. We used default cutoff values for Cook's distance, which varied by the number of model parameters and samples (Love et al., 2014). The significance of DE analysis was tested using two methods. DESeq2 offers a likelihood ratio test (LRT) to examine the effect of one or more variables (with all factor levels) on the normalized count data. LRT was used through the data quality assessment by comparing a full model ( batch effect + tissues) and a reduced model ( batch effect). In all other analyses, Wald-test was applied with independent hypothesis weighting (IHW) as a p-value adjustment (Ignatiadis et al., 2016). To rank genes, we used logarithmic fold change (LFC) values shrunken by the ashr empirical Bayes approach (Stephens, 2017).

\subsubsection{Data quality assessment and detecting outlier samples}

Data quality was examined by (1) inspecting plots of principal component analysis (PCA) which were generated using the prcomp function of the stats v.3.6.3 R package (Core Team R, 2018) based on $\log 2$ normalized counts of all samples, (2) calculating the drop out events that is the number of genes with zero mapped reads in a sample, (3) summarizing the ERCC spike-in statistics and (4) calculating the distribution of Cook's distances in a likelihood ratio test (see below). We considered three statistics regarding the ERCC spike-in controls. First, we calculated the percentage of spike-in control sequences recovered (having at least one mapped read) through RNA-seq. Second, we performed a Pearson correlation between $\log 2$ input ERCC spike-in control concentration and the $\log 2$ read counts and evaluated the Pearson correlation coefficient $\left(\mathrm{R}^{2}\right)$. Third, we determined the value of the lower limit of detection (LLD), which describes the lowest spike-in control concentration, which could be detected through RNA-seq. Cook's distances for each gene in a sample were calculated through a likelihood ratio test using the full model $(\sim$ batch effect + tissues $)$ and the reduced model $(\sim$ batch effect). By inspecting the distribution of Cook's distance of samples, we wanted to detect samples that may contain more distorting data (genes) than other samples do. 


\subsubsection{Defining upregulated genes in hyphal knots and tissue enhanced genes across the development of $\mathrm{C}$. cinerea}

To determine which genes were upregulated at the transition from vegetative mycelium to primary and secondary hyphal knots, we performed three DE analyses using Wald-test with IHW ( $p<0.05$ and shrunken LFC $>1$ ). We compared the (1) vegetative mycelium and the primary hyphal knot, the (2) vegetative mycelium and the nodulus of the secondary hyphal knot, and (3) the vegetative mycelium and the universal veil of the secondary hyphal knot.

To detect tissue enhanced genes, we built on the formula of Sonawane and coworkers (Sonawane et al., 2017):

$$
s_{j}^{(t)}=\frac{\operatorname{med}\left(e_{j}^{(t)}\right)-\operatorname{med}\left(e_{j}^{a l l}\right)}{\operatorname{IQR}\left(e_{j}^{a l l}\right)}
$$

which defines a specificity value ( $s$ ) for a gene $(j)$ in a tissue (t) by calculating the differences between the median expression of gene $\mathrm{j}$ in tissue $\mathrm{t}$ and the median expression of gene $\mathrm{j}$ in all tissues weighted by the interquartile range (IQR) of the gene expression values of all tissues. Similarly to the formula described above, we calculated whether the mean expression of a gene in a tissue was higher than the mean expression of the gene in all tissues in a developmental stage. To do that, we used a GLM model with deviation contrast in the DESeq2 framework (Chambers et al., 1990; UCLA: Statistical Consulting Group, 2020). By introducing the idea of Sonawane and coworkers into the DESeq2 framework, we could take advantage of statistical tools crucial in RNA-seq analysis (Cook's distance, IHW, LFC shrinkage). We defined tissue enhanced genes in a developmental stage as a gene that had significantly higher expression (LFC > 1 and weighted adj. $\mathrm{p}<0.05$ ) than the mean expression value of all tissues. GLM analyses with deviation contrast were performed in four stages (from primordium initial to stage 2 primordium), resulting in ten different analyses (4-4 analyses for universal veil and cap, 1-1 analyses for lamellae and partial veil).

\subsubsection{Functional analysis of upregulated and tissue enhanced genes}

\subsubsection{Compiling a functional annotation database}

To investigate whether certain gene functionalities are characteristic to tissues, we compiled numerous functional annotations into one database. We used Gene Ontology 
(GO) (Ashburner et al., 2000; Carbon et al., 2019), EuKaryotic Orthologous Groups (KOG) (Tatusov et al., 2003) and Kyoto Encyclopedia of Genes and Genomes (KEGG) (Kanehisa, 2000; Kanehisa et al., 2019) terms deposited in the MycoCosm database. We used an InterPro (IPR) database (Mitchell et al., 2019) for C. cinerea compiled by the members of the Fungal Genomics and Evolution lab. We also used annotations based on orthologous genes identified through reciprocal best hit $(\mathrm{RBH})$ analysis (Kristensen et al., 2011) performed in the Fungal Genomics and Evolution lab. In the RBH analysis, functional descriptions of proteins of four model species (Candida albicans (www.candidagenome.org/), Aspergillus nidulans (www.aspergillusgenome.org/), Schizosaccharomyces pombe (www.pombase.org/) and Saccharomyces cerevisiae (https://yeastgenome.org/)) were included in our functional database. YeastPathways terms (https://pathway.yeastgenome.org/) were gathered manually from the list of curated pathways of the database and assigned to $C$. cinerea genes based on RBH analysis. We also included transcription factor and kinase annotations published in Krizsán et al., 2019. Furthermore, we annotated genes by the protein clusters related to hyphal morphogenesis published in Kiss et al., 2019. In this dataset, 17 functional groups were defined by manually curating 519 hyphal-growth related articles. Finally, a manually curated mitosis related gene-set assembled in the Fungal Genomics and Evolution lab was also included in our database.

\subsubsection{Functional enrichment analyses}

We performed functional enrichment analyses on differentially expressed and tissue enhanced genes using IPR, KOG, GO databases and annotations based on Krizsán et al., 2019 (kinases and transcription factors) and Kiss et al., 2019 (hyphal morphogenesis functional groups). GO enrichment analysis was carried out using topGO v.2.38.1 package (Alexa and Rahnenfuhrer, 2020). We used the weigth01 algorithm to deal with the GO graph structure (Alexa et al., 2006) and Fisher's exact test for statistical inference. P-values were adjusted using Benjamini-Hochberg $(\mathrm{BH})$ correction (Benjamini and Hochberg, 1995), and adj. p < 0.05 was considered as a significant result. The enrichment of an IPR, KOG, kinase, transcription factor, hyphal morphogenesis terms were assessed by performing a hypergeometric test coupled with BH correction (adj. $\mathrm{p}<$ 0.05). In the latter cases, we tested whether the proportion of a term in a subset of genes (e.g., tissue enhanced genes) is higher than in the whole dataset. 


\subsubsection{Manually curated functional hypotheses}

Through the transition from vegetative mycelium to hyphal knot stages, we asked whether changes in the hyphal growth pattern can be recognized at the transcriptome level. Therefore, we focused on functional categories such as septation, branching, hyphal fusion, cell wall remodeling, cell wall biogenesis, mitosis and cell cycle control. Furthermore, we wanted to check whether defense-related genes and genes playing a role in forming hydrophobic surface (i.e., 'surface coating' proteins) are more characteristic to the cap, the universal veil and the partial veil than to other tissues. We also wanted to check whether programmed cell death (PCD) related genes are enhanced in the lamellae. We used searching terms (Table 4.) across all compiled annotations and descriptions described above and created lists of upregulated and tissue enhanced genes grouped by functional categories. We manually curated these lists to eliminate hits of poor annotation terms or ambiguous and general descriptions.

Table 4. Functional categories and the searching terms used to filter genes among tissue enhanced and hyphal knot upregulated genes.

\begin{tabular}{|c|c|}
\hline Functional category & Searching term \\
\hline Mitosis & $\begin{array}{l}\text { Mitosis, DNA repair, Replication, Cell division, Spindle Pole } \\
\text { Body }\end{array}$ \\
\hline Cell cycle control & $\begin{array}{l}\text { Checkpoint protein, Cyclin, Growth-arrest-specific protein, } \\
\text { Cycle }\end{array}$ \\
\hline $\begin{array}{l}\text { Hyphal growth pattern (septation, } \\
\text { branching, fusion, polarized } \\
\text { growth) }\end{array}$ & $\begin{array}{l}\text { Septin, Branching, filamentous growth, cell polarity, } \\
\text { anastomosis, STRIPAK }\end{array}$ \\
\hline Cell wall biogenesis & $\begin{array}{l}\text { chitin synthase activity, cell wall assembly, Conidiation-specific } \\
\text { protein, (GPI)-anchored proteins }\end{array}$ \\
\hline Cell wall remodeling & $\begin{array}{l}\text { chitinase, cellulase, glycoside hydrolase, polysaccharide lyase, } \\
\text { glycosyl transferase, }\end{array}$ \\
\hline Surface Coating & Hydrophobin, Wax synthase, Cerato-platanin \\
\hline Defense & $\begin{array}{l}\text { Copsin/Plectasin/ Micasin, lectins, pore-forming aerolysin, } \\
\text { Peptidase inhibitor I66 }\end{array}$ \\
\hline Programmed cell death & $\begin{array}{l}\text { caspase, death, apoptosis, DNM1, BCL, Bax, CasA, CasB, AIF, } \\
\text { EndoG }\end{array}$ \\
\hline
\end{tabular}




\subsubsection{Genomic phylostratigraphy}

\subsubsection{Phylostratigraphic analysis}

To reveal the evolutionary origin of tissue enhanced genes we performed phylostratigraphic analyses using the genomic data of 441 species of the Fungi regnum. One-to-one ortholog data of Coprinopsis cinerea was available in the Fungal Genomics and Evolution lab in an RBH database mentioned above. A phylogenomic tree was also inferred by the members of the Fungal Genomics and Evolution lab using 344 genes and FastTree. We used this phylogenomic tree to assign age indexes (PS, 'phylostrata') to the nodes along the evolutionary route from Coprinopsis cinerea to the root of the tree. Using the RBH database and the phylostratum annotations, we traced back the last common ancestor of all $C$. cinerea genes and assigned age indexes to them. Then we checked whether an age index is more abundant in any of the tissue enhanced gene sets. To do that, we carried out an enrichment analysis based on the ratio of an age index in a gene set and all genes of the $C$. cinerea genome (hypergeometric test, $\mathrm{BH}$-adj. $\mathrm{p}<0.05$ ).

3.2.6.2. Determining the first occurrence of a character state along the backbone

A maximum parsimony based ancestral character state reconstruction (ASR) analysis was performed to infer the possible occurrences of six morphological characters (enclosed development, cap, increased hymenophore surface area, universal veil, and partial veil) along the evolutionary route of $C$. cinerea. We used the hsp_max_parsimony function from the castor v.1.5.5. R package (Louca and Doebeli, 2018) which used an efficient and fast algorithm allowing to define missing data and a transition cost matrix. We used a transition cost matrix to mimic the evolutionary patterns we found through the BayesTraits analyses. First, we calculated the mean of the transition rates inferred through Bayesian and ML analysis and took the reciprocal of the transition values. Then we shifted all values by the maximum of the reciprocal matrix to create a gap between no cost (diagonal of the cost matrix) and the lowest cost transition. The function handled ambiguous character states as unknown states. 


\subsection{Data availability}

All scripts and codes are available from the authors upon request if the direct availability was not mentioned otherwise in the text. Trees and alignments have been deposited in Dryad Digital Repository (accession number: doi:10.5061/dryad.gc2k9r). The electronic appendix is available on DVD supplemented the printed version of the thesis, or online at the following link:

https://www.dropbox.com/sh/mphbfmqauoxo3lv/AACqdBPaq7zh5nbA19xxQGW_a?dl $\underline{=0}$ 


\section{Results}

\subsection{Comparative phylogeny of mushroom-forming fungi}

\subsubsection{Inferring phylogenetic trees and chronograms}

To examine the evolution of complex morphologies in fungi we decided to analyze mushroom-forming fungi (Agaricomycetes) which includes more than 20,000 species with numerous type of complex fruiting bodies. To do that, we attempted to gather specimens throughout the world and reconstruct a robust phylogeny which could represent the evolutionary history of Agaricomycetes and two other classes, the Dacrymycetes and Tremellomycetes as outgroups. We gathered specimens from undersampled geographical regions such as Africa, Asia, Australasia, or South America, therefore our dataset contains species from every continent except Antarctica. The superalignment of the 5,284 species contained 5,737 nucleotide characters in total, which was divided into the three partitions (tefl- $\alpha$, nrLSU, and rpb2) as 1,052, 2,768 and 1,917 characters, respectively. All the translation elongation factor 1- $\alpha$ (tef1- $\alpha$ ) the nuclear large ribosomal subunit (nrLSU) and the RNA polymerase II second largest subunit $(\mathrm{rpb} 2)$ are widely used phylogenetic markers and could be used to infer both interspecific and higher level of relationships as well (Roger et al., 1999; Hibbett et al., 2007). We inferred $245 \mathrm{ML}$ trees and tested if this set of phylogenies covers a sufficiently plausible set of topologies given the alignment. Robinson-Foulds (RF) distances were calculated for each pair of trees, and the average, minimum and maximum were plotted as a function of the number of trees (Figure 4.). All three statistics reached saturation, meaning that the topological variety would not increase by adding further trees to this set. The main topology of the trees was in concordance with previous studies (Hibbett, 2006; Floudas et al., 2012; Kohler et al., 2015; Nagy et al., 2016), therefore we think our phylogenies represent a set of reliable evolutionary histories for mushroom-forming fungi. 


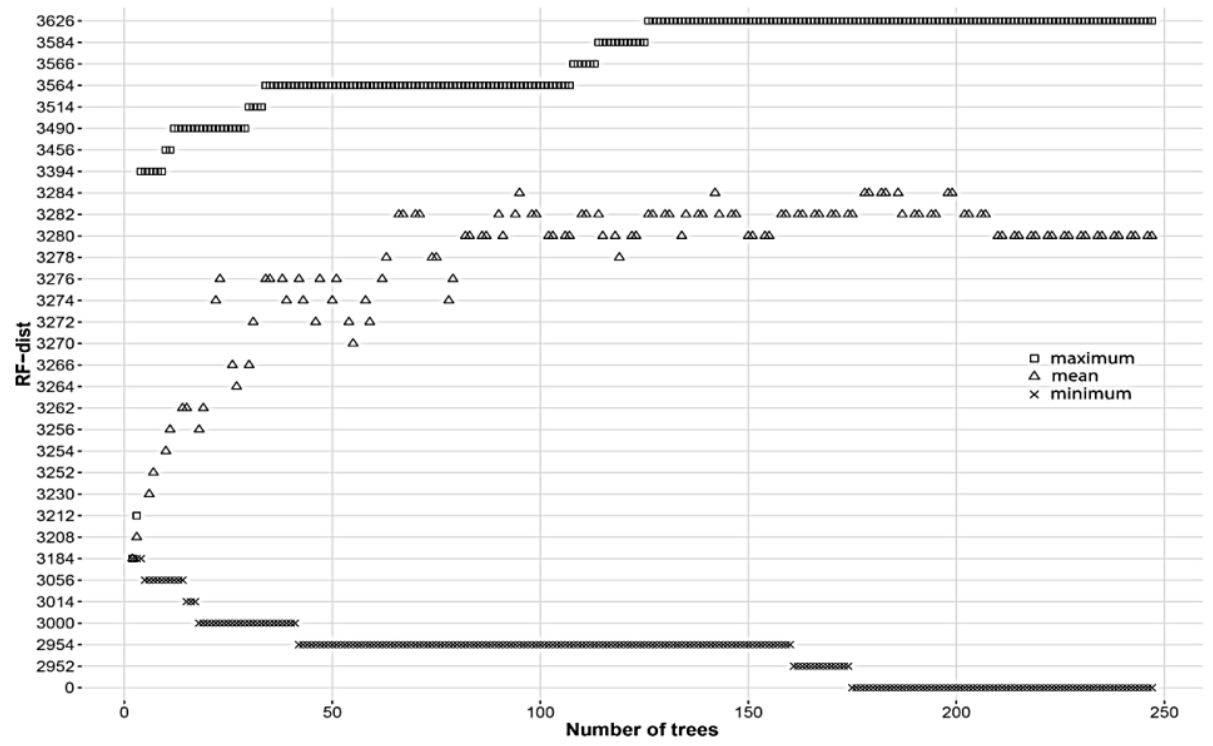

Figure 4. The rolling average, maximum and minimum values of $R F$-distance values across the 245 phylogenetic trees. RF-distance (y axis) express the dissimilarity of tree topologies without considering branch length. Therefore, the higher the RF-distance value, the more the differences are between trees in a pair-wise comparison.

We chose ten phylogenies out of the 245 ML trees which were used in our twostep time calibration procedure. We used a stratified random sampling to cover the topological diversity of all ML trees. Through time calibration of the ten 5,284-species trees, both PhyloBayes and FastDate analyses reached convergence; therefore, the posterior samples represent the most probable set of ages given our data. We chose eight constraints on the age of the MRCA of recent species through the time inference based on fossil data (Table 1.). We could use all the eight fossil calibration points in our analyses with confidence because the fossil cross-validation procedure showed that the selected fossils are not in conflict with each other (Figure 5.). Based on our time calibration, the origin of most of the order- or subclass-level clades dated to the Jurassic period (200-145 Ma ago) such as Cantharellales, Hymenochaetales, Russulales, Agaricales, Phallomycetidae and Agaricomycetidae (Table 5., Electronic Appendix 3). 


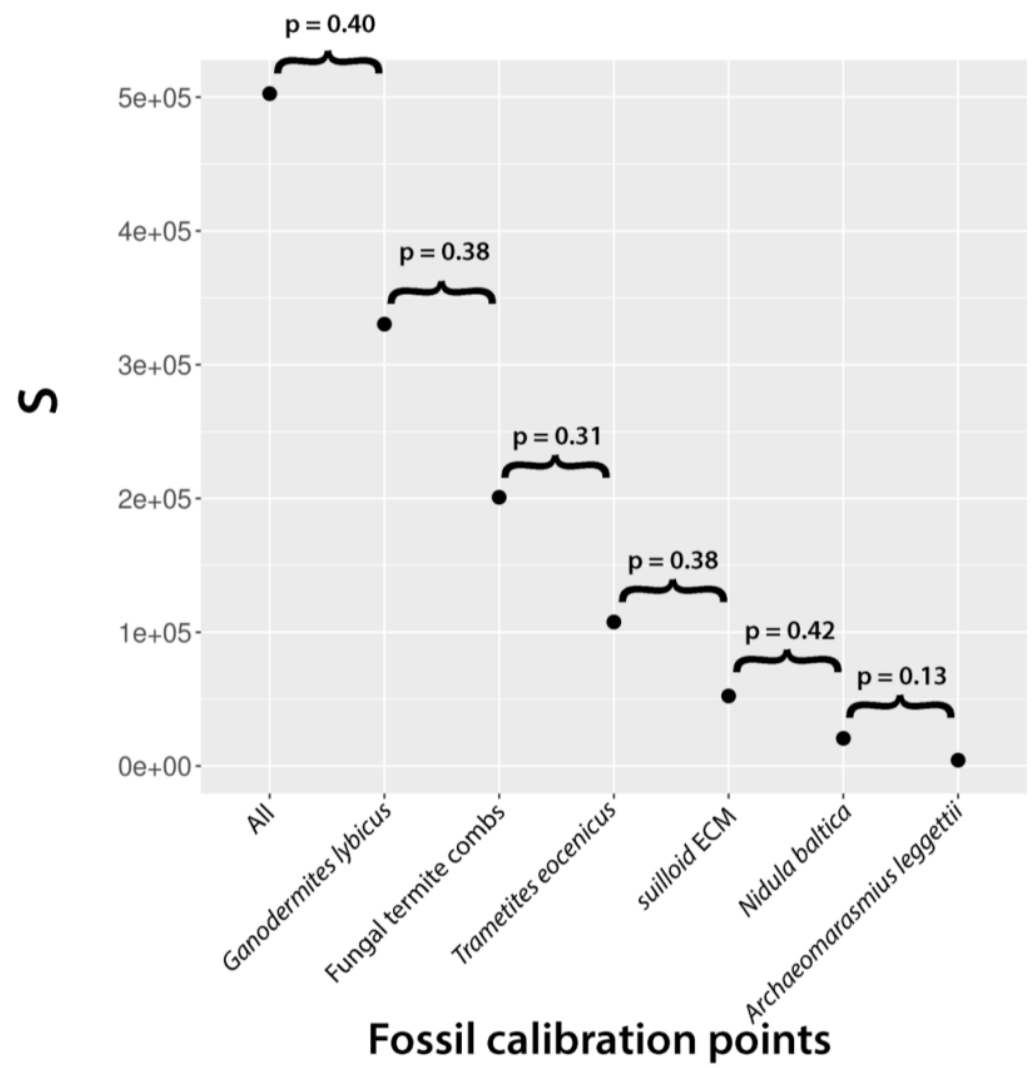

Figure 5. The effects of removing fossil calibration points on the average squared deviation ( $s$ ) between molecular and fossil age estimates for all calibration points. There was no significant drop in the variance (one-tailed F-test, $p<0.05$ ) in any comparison indicating no conflicts between the eight time-constraints

To verify our age estimates, we performed molecular clock dating using genomic data of 105 species. The concatenated alignment contained 70 conserved genes and 18,917 amino acid characters. The momctree analysis reached convergence and we found similar ages to the 5,284-species time inference based on the summary of 2,000 timecalibrated trees from the posterior (Figure 6., Table 5.). 
Table 5.. The average age of the main clades inferred by a two-step Bayesian time calibration analysis using ten phylogenies consists of 5,284 species (5,284-species chronogram) and by mcmctree on the genomic data of 105 species (Genome based chronogram). 95\% HPD $=95 \%$ highest posterior density.

\begin{tabular}{|l|c|c|c|c|c|}
\hline \multirow{2}{*}{ Clade } & \multicolumn{2}{|c|}{$\begin{array}{c}\mathbf{5 , 2 8 4 - s p e c i e s} \\
\text { chronogram }\end{array}$} & \multicolumn{2}{c|}{$\begin{array}{c}\text { Genome based } \\
\text { chronogram }\end{array}$} & \multirow{2}{*}{ \# of species } \\
\cline { 2 - 5 } & $\begin{array}{c}\text { Average age } \\
\text { (Ma) }\end{array}$ & SD & $\begin{array}{c}\text { Average age } \\
\text { (Ma) }\end{array}$ & $\begin{array}{c}\text { 95\% } \\
\text { HPD }\end{array}$ & \\
\hline Agaricomycetidae & 182 & 9 & 180 & $165-198$ & 3,806 \\
\hline Agaricales & 172 & 8 & 173 & $158-190$ & 3,369 \\
\hline Boletales & 139 & 7 & 138 & $126-152$ & 392 \\
\hline Amylocorticiales & 100 & 6 & - & - & 18 \\
\hline Atheliales & 87 & 5 & 85 & $72-99$ & 16 \\
\hline Lepidostromatales & 81 & 7 & - & - & 9 \\
\hline Polyporales & 136 & 6 & 138 & $126-152$ & 369 \\
\hline Thelephorales & 100 & 5 & - & - & 18 \\
\hline Russulales & 147 & 14 & 135 & $121-151$ & 316 \\
\hline Gloeophyllales & 82 & 6 & 47 & $41-54$ & 17 \\
\hline Jaapiales & 42 & 2 & - & - & 2 \\
\hline Corticiales & 101 & 6 & - & - & 22 \\
\hline Hymenochaetales & 152 & 14 & 176 & $157-197$ & 235 \\
\hline Phallomycetidae & 174 & 18 & 146 & $130-164$ & 188 \\
\hline Trechisporales & 86 & 5 & - & - & 10 \\
\hline Auriculariales & 134 & 15 & 76 & $65-90$ & 31 \\
\hline Sebacinales & 133 & 7 & 88 & $76-103$ & 38 \\
\hline Cantharellales & 184 & 44 & 246 & $223-277$ & 85 \\
\hline Dacrymycetes & 133 & 5 & 78 & $67-91$ & 41 \\
\hline Tremellomycetes & 165 & 13 & 167 & $142-193$ & 108 \\
\hline
\end{tabular}




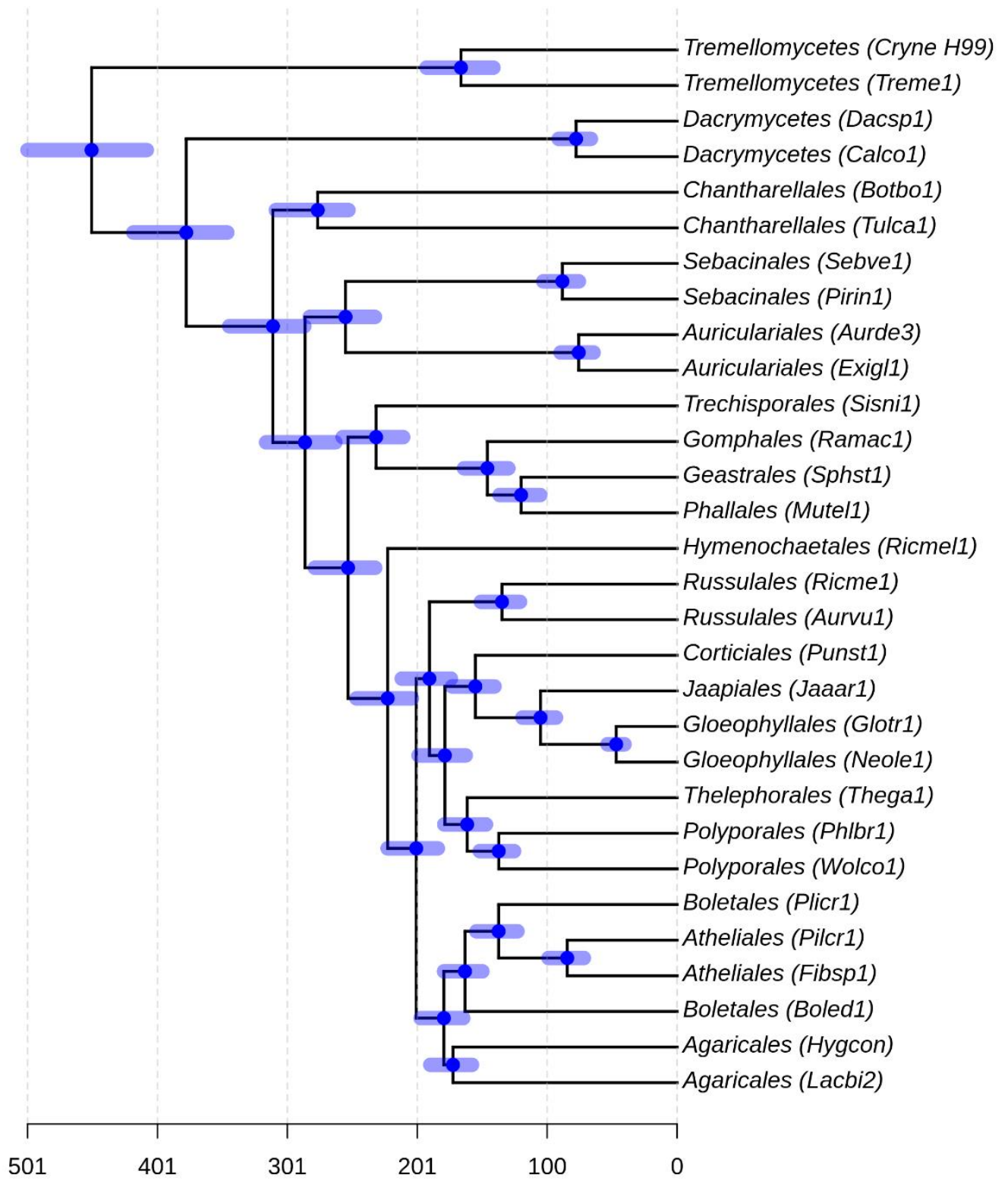

Figure 6. The result of the genome-based time calibration using mcmctree. The depicted chronogram is the modified version of the 105 species tree, because only the order level clades were kept focusing on the main age estimates. Blue bars around the nodes represent the 95\% highest posterior densities (HPD).

Both the genome- and phylogeny-based inferred ages were older than it was reported by previous studies (Floudas et al., 2012; Kohler et al., 2015). Therefore, to discover the underlying causes our genomic data were reanalyzed. We wanted to uncover whether the placement of the fossils and/or the number of fossils used in this study and in Kohler et al. 2015 could cause differences in time estimates. We used the r8s program where the cross-validation procedure determined 10 or 100 as the optimal smoothing parameter $(\lambda)$ for penalized likelihood analysis, therefore we used the $\lambda=10$ parameter in further analyses. By comparing the results of three r8s analyses differed in the set of 
calibration points and their positions on the tree, we found that molecular age estimates are primarily driven by the precision of the placement of fossil calibrations on the tree and to a smaller extent by the set of fossil calibration points and the dataset used. Using the 'default calibration scheme' (eight fossil calibration points as it is applied in the 5,284species dataset, Table 1.), resulted in slightly younger ages relative to the results of the 5,284-species time inference and to that of the genome-based age estimates using mcmctree (Table 6.). We also applied the 'Kohler et al. calibration scheme 1' to our data which represents the same time constraints used in the study of Kohler et al. 2015 (Suilloid ECM and Archaeomarasmius leggettii). The results of the 'Kohler et al. calibration scheme 1' showed much younger time estimates than any of the analyses with 'default calibration scheme', but older than in the original paper. The analysis with the 'Kohler et al. calibration scheme 1' suggests that purely the increased number of genomes could cause older age estimates. Furthermore, by applying the 'Kohler et al. calibration scheme 2', we found as old age estimates as in the case of the 5,284 species dataset and the momctree genomic analysis. The latter calibration scheme differs from the 'Kohler et al. calibration scheme 1' in that the two fossil calibration points constrained the crown node instead of the stem node of Suillaceae and Marasmioid clade. Constraining the crown node of Suillaceae was not possible a few years ago because of the lack of crucial genomes. 
Table 6. Comparison of the results of different molecular dating analyses.

Estimated ages of different clades of

Molecular dating analyses Agaricomycotina (Ma)

\begin{tabular}{|c|c|c|c|c|c|c|}
\hline Calibration scheme & Methods & $\begin{array}{c}\text { Trees were } \\
\text { analyzed }\end{array}$ & Agaricales & Boletales & $\begin{array}{l}\text { Agarico- } \\
\text { mycetidae }\end{array}$ & Agaricomycetes \\
\hline $\begin{array}{l}\text { Analyses of Kohler } \\
\text { et al. } 2015\end{array}$ & r8s & - & 108 & 84 & 125 & 294 \\
\hline $\begin{array}{l}\text { Present study, Kohler } \\
\text { et al. calibration } \\
\text { scheme } 1 \\
\end{array}$ & r8s & $\begin{array}{l}\text { Genomic data } \\
\text { of } 105 \text { species }\end{array}$ & 126 & 117 & 131 & 232 \\
\hline $\begin{array}{l}\text { Present study, Kohler } \\
\text { et al. calibration } \\
\text { scheme } 2\end{array}$ & r8s & $\begin{array}{l}\text { Genomic data } \\
\text { of } 105 \text { species }\end{array}$ & 169 & 166 & 176 & 269 \\
\hline $\begin{array}{l}\text { Present study, } \\
\text { Default calibration } \\
\text { scheme }\end{array}$ & r8s & $\begin{array}{l}\text { Genomic data } \\
\text { of } 105 \text { species }\end{array}$ & 156 & 150 & 162 & 271 \\
\hline $\begin{array}{l}\text { Present study, } \\
\text { Default calibration } \\
\text { scheme }\end{array}$ & mcmctree & $\begin{array}{l}\text { Genomic data } \\
\text { of } 105 \text { species }\end{array}$ & 175 & 166 & 182 & 317 \\
\hline \multirow{10}{*}{$\begin{array}{l}\text { Present study, } \\
\text { Default calibration } \\
\text { scheme, } 5284 \text { species } \\
\text { tree }\end{array}$} & \multirow{10}{*}{$\begin{array}{l}\text { Phylobayes } \\
+ \text { FastDate }\end{array}$} & tree 8 & 182 & 153 & 192 & 347 \\
\hline & & tree 12 & 174 & 136 & 184 & 353 \\
\hline & & tree 16 & 160 & 134 & 174 & 279 \\
\hline & & tree 53 & 173 & 136 & 192 & 323 \\
\hline & & tree 88 & 175 & 141 & 190 & 353 \\
\hline & & tree 165 & 167 & 133 & 176 & 300 \\
\hline & & tree 170 & 181 & 148 & 191 & 387 \\
\hline & & tree 188 & 179 & 135 & 187 & 351 \\
\hline & & tree 216 & 162 & 135 & 178 & 372 \\
\hline & & tree 222 & 172 & 139 & 187 & 346 \\
\hline
\end{tabular}

\subsubsection{Accounting for non-random and incomplete taxon sampling}

Next, we wanted to scrutinize the macro-evolutioanry patterns of Agaricomcetes using PCMs and our robust phylogenies and chronograms. However, many macroevolutionary frameworks assume that the phylogenetic tree under scrutiny contains all the existing species of the taxa examined. This is the case with the BAMM and the SSE (state speciation and extinction) models, therefore we introduced correction strategies into our analyses. We considered the taxonomically valid species in the Species Fungorum database as they represent all contemporary species. Overall, 35,470 species were found to belong to the Dacrymycetes, Tremellomycetes, and Agaricomycetes classes based on the Species Fungorum database in 2016. We used this data to calculate genus-wise sampling fractions and found that 390 genera (out of 782) were significantly over- (365) or undersampled (25). We created a 'skeletal tree' where just a portion of all existing species can be found, but the distribution of these species is proportional at higher 
taxonomical levels to the 'full tree'. We randomly and iteratively deleted species from genera, which contained proportionally more species in our dataset than in the Species Fungorum dataset (hypergeometric test, $\mathrm{p}<0.05$ ). This procedure resulted in the deletion of 1083 species but also the reduction of the number of over- and undersampled genera from 365 and 25 to 112 and 12, respectively. The remaining oversampled genera mostly contained 1 or 2 species, meaning that we could not mitigate oversampling without removing the genus itself from the dataset. In preliminary analyses, we found congruent results of applying no correction at all or using a 'skeletal tree' or built-in sampling corrections of the programs. Although we detected no apparent effect of sampling bias corrections on the results, we applied the built-in corrections of the SSE and BAMM models in further analyses, because we wanted to fulfill all the theoretical assumptions of the models.

\subsubsection{Trait independent diversification of mushroom-forming fungi}

After we asserted that our phylogenetic inference is robust and the sampling fractions of species would not affect significantly PCMs, we first explored the speciation and extinction patterns through the evolution of mushroom-forming fungi. We performed a BAMM analysis with 100 million MCMC generations using the ten time-calibrated trees. We found that the likelihood values reached convergence, which was supported by Geweke's statistics as well ( $p>0.05$, Electronic Appendix 2). The number of shifts and the rate-shift frequency ('event rate') parameters also showed saturation (Geweke's statistics, $p>0.05$ ) in the post-burn samples and most of the trees. Despite the high number of posterior samples generated, only the 'event rate' parameter possessed sufficiently high effective sample size (ESS) value (> 200), while other parameters had on average a moderate ESS value ( 100). This behavior of ESS statistics suggests that a large tree could result in an enormous number of evolutionary scenarios, which could make a macro-evolutionary analysis challenging to converge.

By analyzing speciation and extinction rates through time we found a substantial increase in speciation and diversification rate (speciation minus extinction rate) starting abruptly at around $180 \mathrm{Ma}$ ago in the Jurassic period. This pattern was pronounced in all the ten chronograms, and it was also supported by the lineages through time plots (Figure 7.). The growth of diversification rate continued until the present time, where we noticed a flattened pattern meaning an elevated but not increasing diversification rate. 


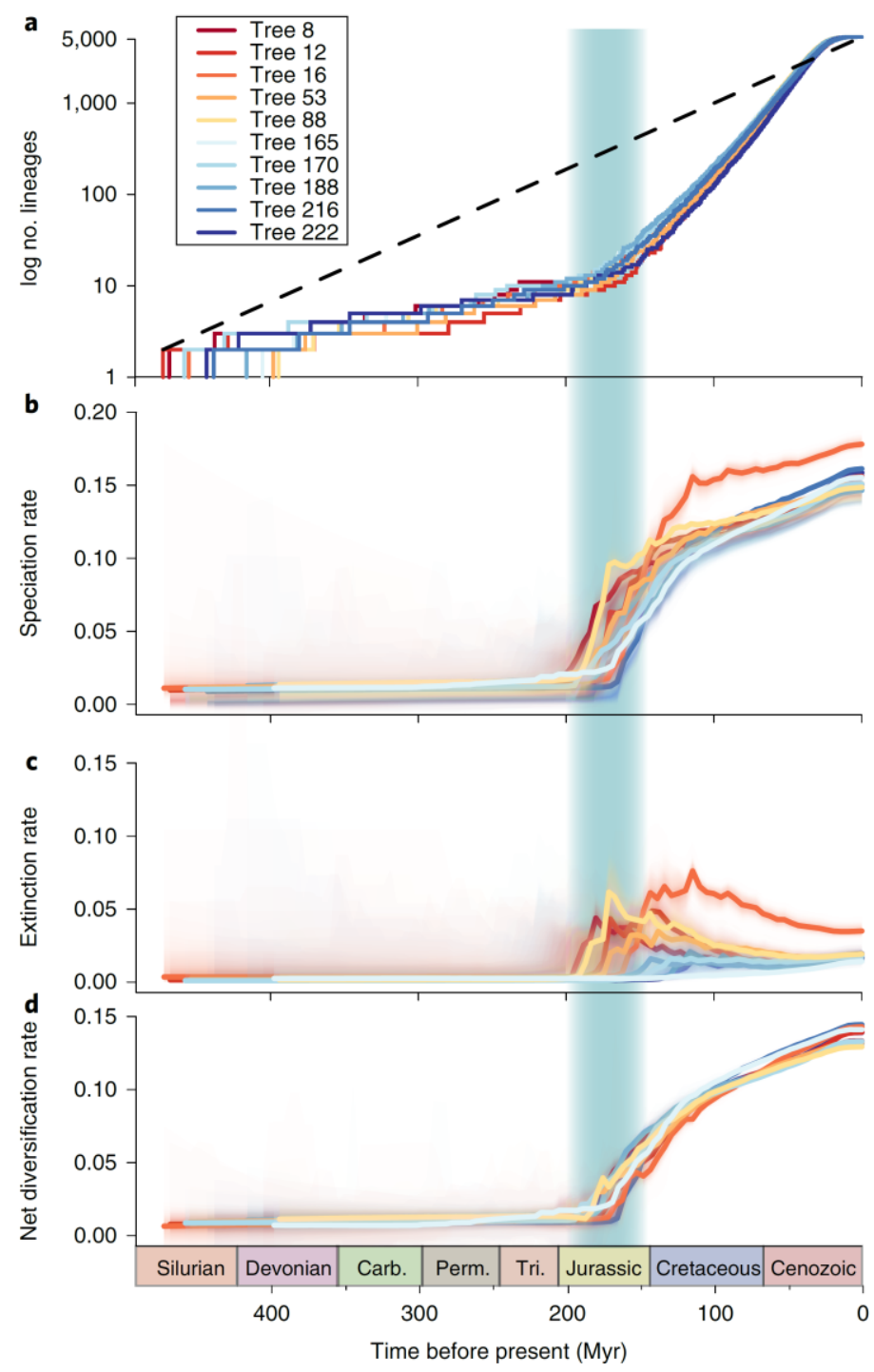

Figure 7. Broad macro-evolutionary patterns of mushroom-forming fungi. (a) Lineages through time plot of the ten time-calibrated trees. $(b-d)$ Rates through time plots depicting the mean evolutionary rates in a time period through lineages. (b) Speciation rate (c) extinction rate (d) diversification rate. The blue sector shows the Jurassic period.

\subsubsection{Extinction events through the evolution of Agaricomycetes}

The speciation rate acceleration shown above was followed by a slight increase in the extinction rate $\sim 150 \mathrm{Ma}$ ago (Figure 7 .). This rate pattern may imply the presence of an extinction event at the end of the Jurassic period, therefore we used the CoMET model to test this hypothesis. The CoMET model is suitable to test an evolutionary hypothesis by comparing two models with and without a mass extinction event. Our analysis on ten chronograms showed that models with a mass extinction event were preferred over models without mass extinction events (Table 7., mean Bayes factor: 25.7). These model tests suggest that our data support the presence of at least one mass extinction event during the evolution of mushroom-forming fungi. 
Table 7. Results of MCMC analyses under models with and without mass extinction events. The null model (MO) was a constant-rate birth-death CoMET model with mass extinction events allowed and the alternative model (M1) was the same as MO, but without a mass extinction event. Bayes factors were calculated from the marginal likelihoods of the two models, using stepping stone simulation.

\begin{tabular}{cccc} 
Tree ID & \multicolumn{2}{c}{$\begin{array}{c}\text { Marginal log likelihood values } \\
\text { Mass extinction }\end{array}$} & $\begin{array}{c}\text { Bayes } \\
\text { factor }\end{array}$ \\
\hline 8 & -24116 & -24128 & 23 \\
12 & -23930 & -23947 & 34 \\
16 & -23543 & -23554 & 21 \\
53 & -23826 & -23838 & 25 \\
88 & -24032 & -24043 & 22 \\
165 & -23638 & -23648 & 21 \\
170 & -24049 & -24065 & 31 \\
188 & -24058 & -24071 & 26 \\
216 & -23480 & -23494 & 27 \\
222 & -23937 & -23951 & 27
\end{tabular}

By using an rjMCMC implementation of the CoMET model, we inferred the number of mass extinction events, the speciation rate and the extinction rate changes through time. This analysis was robust to the choice of priors on the expected number of mass extinction events, expected rate changes and survival probability. All numerical parameters reached high effective sample size values (ESS > 500), and showed convergence according to Geweke's statistics ( $p>0.05$ ). Only likelihood values did not pass Geweke's test in 7 out of 10 analyses, although they appeared to be converged based on the likelihood diagnostic plots (Electronic Appendix 2). The interval specific parameters converged in every time interval (Geweke's statistics: $p>0.05$ ) and had high ESS values (>500). The rjMCMC analyses showed a mass extinction event during the Jurassic period with strong support $(\operatorname{lnBF}>6)$ in nine out of the ten trees. A second mass extinction event was supported in the Triassic period based on three trees (Figure 8.). Overall, analyses using BAMM and two implementations of the CoMET model supported one mass extinction event at the end of the Jurassic period. 


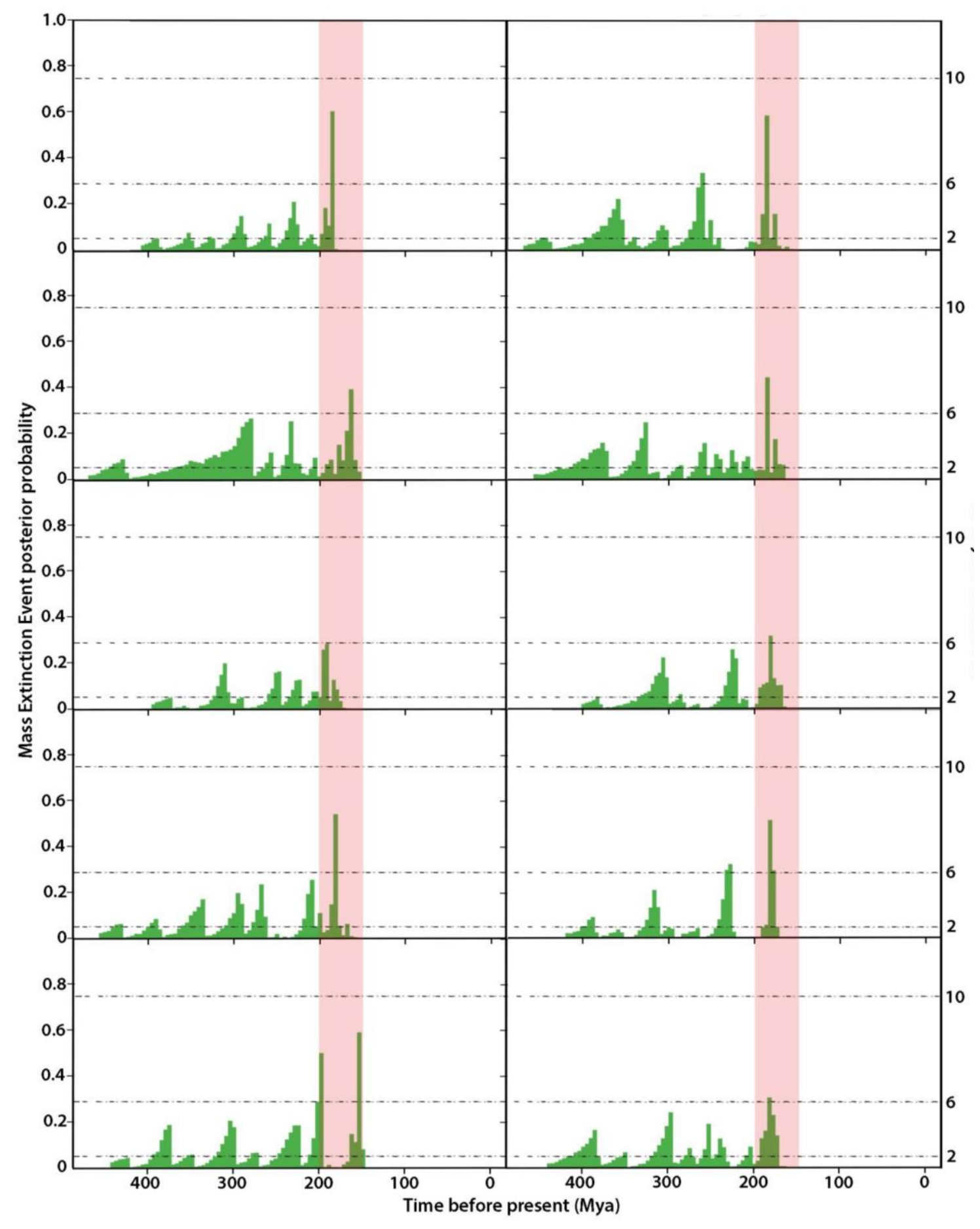

Figure 8. Inferred mass extinction events using rjMCMC implementation of the CoMET model. The left y-axis represents the posterior probability of a mass extinction event in a given time frame. Dashed lines indicate ln Bayes factor thresholds of significance. We considered $\ln$ Bayes factor $>6$ as strong support for an event (right y-axis). The ten graphs represent the results of the analyses of ten chronograms. The shaded red region shows the interval of the Jurassic period. 


\subsubsection{Main shifts in the diversification rate of mushrooms}

Using the BAMM analysis, we inferred shifts in the diversification rate, which could help to explain the speciation and extinction rate patterns we described above. We found that all the 5001 post-burn posterior samples comprised a unique shift configuration. This high variability of shift configurations could be caused by the size of the phylogenies resulting in several statistically equivalent but slightly different evolutionary scenarios. Therefore, we decided to include taxonomical information into the rate shift analysis. First, we summarized all the core shifts (prior-to-posterior marginal odds ratio > 5) in each tree and compared their taxonomical position across the ten chronograms. We took into account small variations in the position of the core shifts because many shifts occurred on adjacent branches around the MRCA of a group. This phenomenon could generate many different shift configurations, although they could correspond to the same evolutionary signal. In these cases, we chose a core shift with the highest posterior probability. Then, we summarized those core shifts which occurred at the same taxonomical position in at least four chronograms out of ten and had high statistical support (mean posterior probability $>0.5$ across the ten chronograms). We refer to these statistically and taxonomically supported rate shifts as congruent core shifts. We also took into account distinct shift configurations by detecting mutually exclusive congruent core shifts from our analyses. Mutually exclusive rate shifts can happen when an evolutionary event can be explained by either a rate increase or a rate decrease on adjacent branches and in different shift configurations. Therefore, we considered two shifts mutually exclusive if only a negligible co-occurrence was present across shift configurations and the direction of the diversification rate changes was opposite. In the end, we found 85 congruent and mutually non-exclusive core shifts that were highly supported by our data (Figure 9., Electronic Appendix 2).

We inferred congruent core shifts in the MRCA of many order-level clades, including Cantharellales, Sebacinales, Auriculariales, Hymenochaetales and Agaricales. Deeper in the tree, we detected rate shifts at the MRCAs of the clades Tremellomycetes, Dacrymycetes, Phallomycetidae, Agaricomycetidae, the monophyletic group formed by Russulales, Polyporales, Thelephorales, Corticiales, Gloeophyllales, Jaapiales and the clade formed by Boletales, Amylocorticiales, Lepidostromatales and Atheliales. Most of the congruent core shifts (57) were inferred in the Agaricales. Only nine clades out of the 85 contained species having a fruiting body without cap and 57 clades exclusively 
contained species that have pileate fruiting bodies (Electronic Appendix 2). 45 shifts out of 57 described a positive change in the speciation rate, suggesting that morphological traits related to the pileus could positively affect the diversification rate of species.

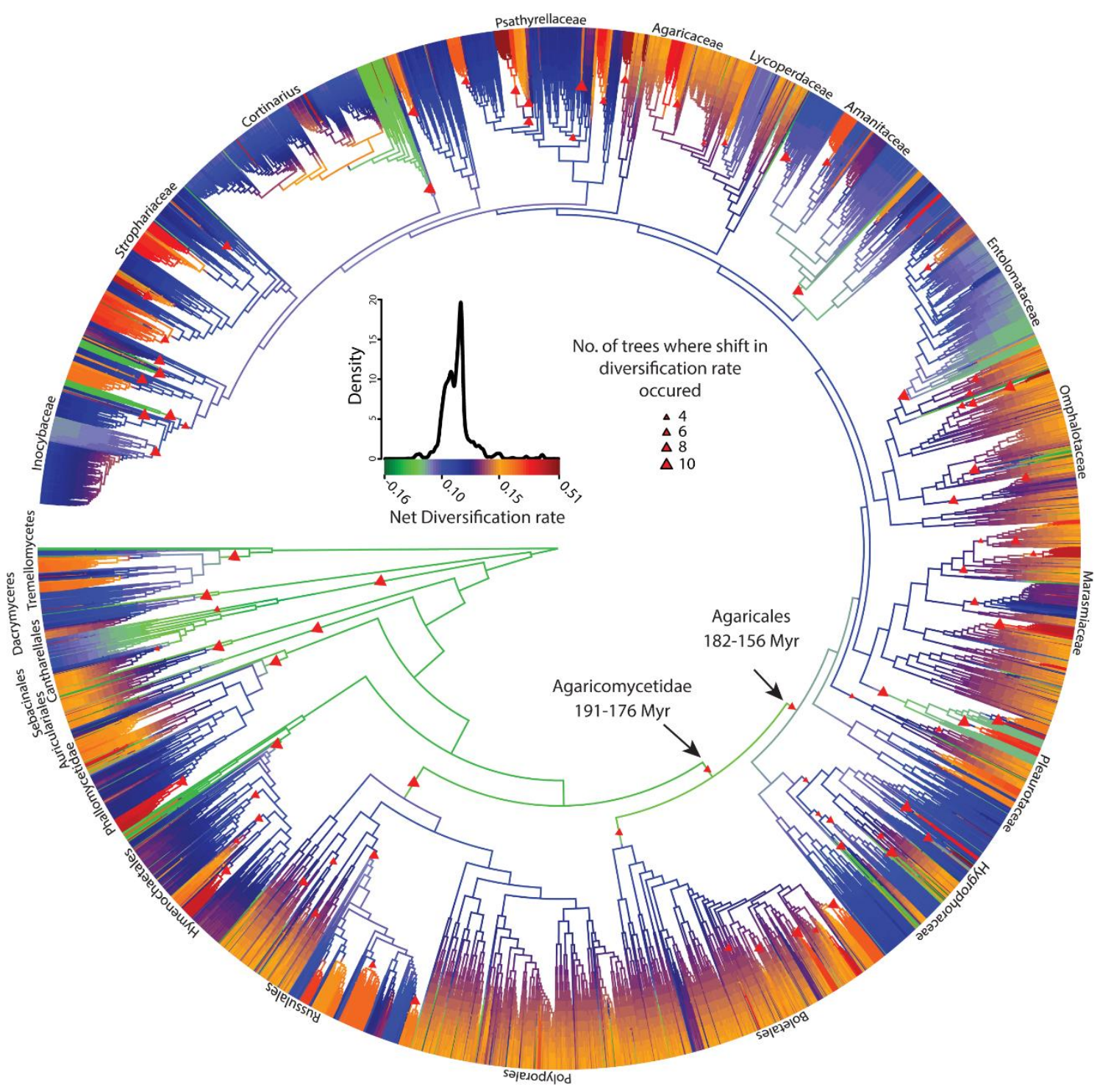

Figure 9. A chronogram of 5,284 species. Branches are colored according to the branch specific diversification rate. Triangles show 85 significant shifts in diversification rate. Several shifts occurred at the stem node of orders and of Agaricomycetidae. $67 \%$ of the shifts were found in the Agaricales, predominantly in the Paleogene.

\subsubsection{Reconstructing ancestral character states of main fruiting body types}

To uncover the morphological traits which could contribute to the diversification rate changes described above, we first examined the evolution of main morphological types (Figure 3.): (1) no fruiting body production, (2) resupinate, (3) pileate, (4) cyphelloid, (5) gasteroid/secotioid and (6) coralloid/clavarioid fruiting body types. Stochastic character mapping performed on the ten chronograms gave similar results, 
therefore, we could summarize the ancestral character states derived from ten analyses on one states through time (STT) plot (Figure 10.). We found that resupinate mushrooms and species without fruiting bodies were dominant at the beginning of the evolution of mushroom-forming fungi. Complex fruiting body types (pileate, cyphelloid, gasteroid/secotioid, coralloid/clavarioid) appeared in the Jurassic period. Then the cumulative posterior probability of the pileate fruiting body type gradually increased and became the most dominant morphology among mushroom-forming fungi. This result is in concordance with the rate shift inference in BAMM analysis, where most of the congruent core shifts were related to a clade consisting of species with pileate fruiting bodies.

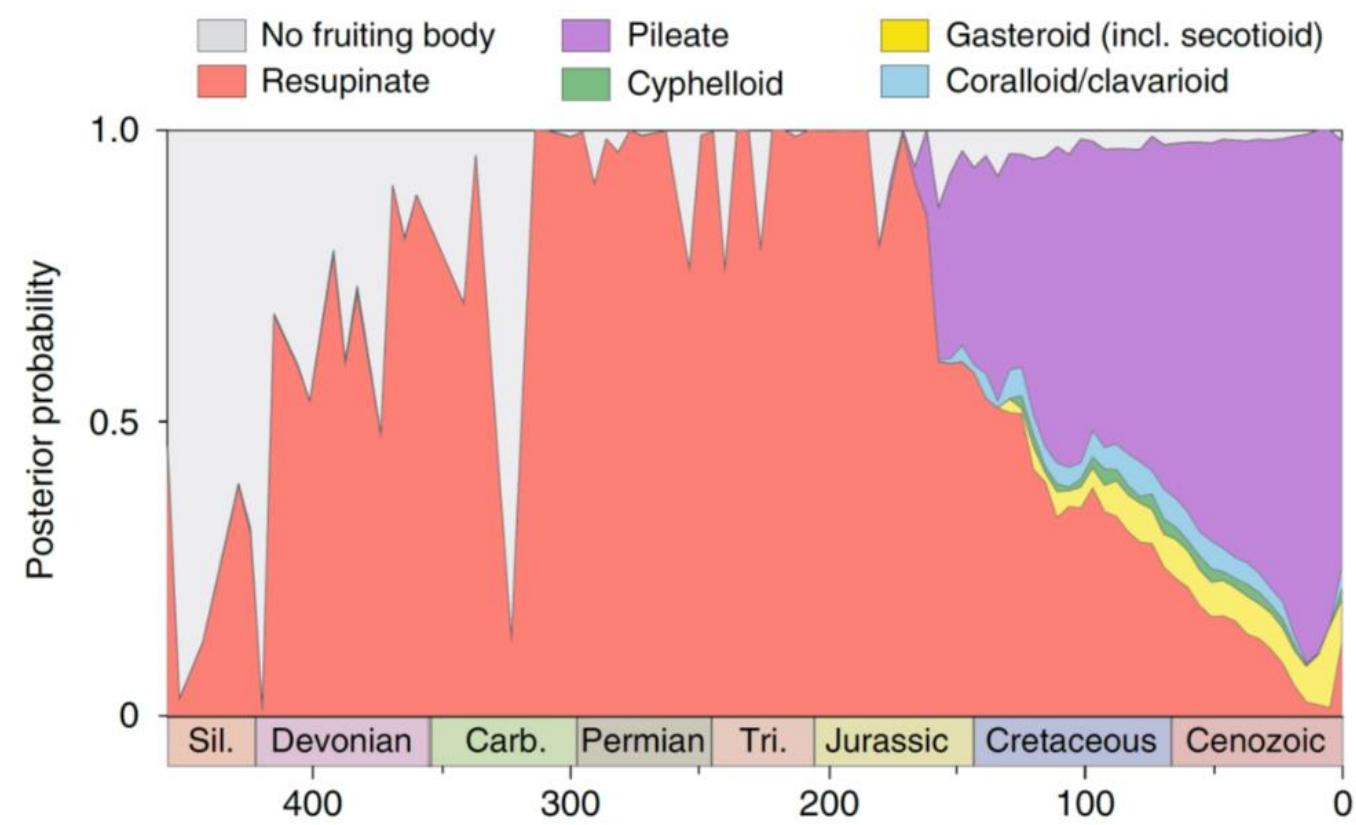

Figure 10. States through time plot of six main fruiting body types across mushroom-forming fungi.

\subsubsection{Morphological character evolution of mushroom-forming fungi}

Analyses above showed an increased speciation rate of clades dominated by species with pileate fruiting body type and the prevalence of pileate fruiting body type after the Jurassic period. Based on these results, we hypothesized that morphological characters associated with pileate fruiting bodies could be advantageous for species through the evolution of mushroom-forming fungi. Therefore, to explain, at least partially, the evolutionary patterns described above, three main morphological traits of fruiting bodies were analyzed in MK (BayesTraits analyses) and SSE (diversitree R package) character evolution models: (1) the presence of the cap, (2) enclosed 
development and (3) increased hymenophore surface area. We further disentangled the enclosed development trait by examining the presence or the absence of the universal veil or the partial veil as protective tissues (Electronic Appendix 1, Figure 3.).

\subsubsection{Macro-evolution of the pileate morphology}

First, we asked whether solely cap formation can be advantageous for species through evolution. The BayesTraits analyses showed that gains of the pileate morphology were favored across mushroom-forming fungi (Figure 11., Electronic Appendix 4). The average transition rate from the non-pileate towards the pileate state (q01) was 6.9-51.5 times higher than in the reverse direction (q10) depending on the method (ML or MCMC).

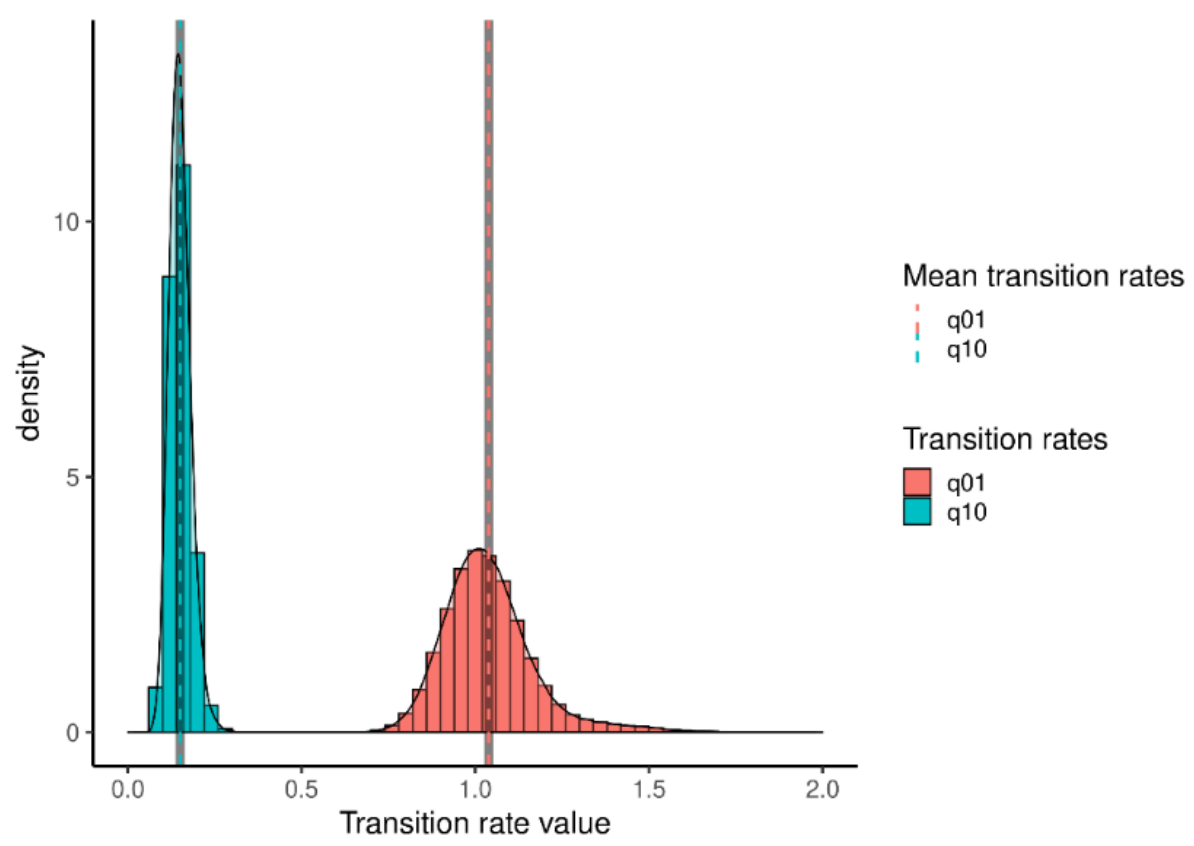

Figure 11. The distribution of the inferred transition rates between pileate and non-pileate states using BayesTraits Bayesian MCMC analysis. $q 01=$ Transition rate from non-pileate to pileate state. $q 10=$ transition rate from pileate to non-pileate state.

This inequality of the two transition rates was also supported by the likelihood ratio tests and Bayes factors. Yet, the reverse transition also proved to be a significant component of our model given on our data (Electronic Appendix 4). These analyses suggest that the convergent evolution of the cap was frequent, implying the evolutionary importance of this trait. However, the evolution of mushroom-forming fungi cannot be explained without the loss of this structure as model tests showed. All these findings were supported by the analyses performed under the BiSSE model (Electronic Appendix 5). By analyzing trait dependent speciation and extinction rates under the BiSSE model, we found that species with pileus had higher diversification rates than species without it 
(Figure 12.). The higher diversification rate was mainly driven by the speciation rate, because it was significantly higher in lineages with pileus than in other lineages (on average 1,39 times higher, LR-test, $\mathrm{p}<0.001)$, but the extinction rates did not differ significantly (Electronic Appendix 4).

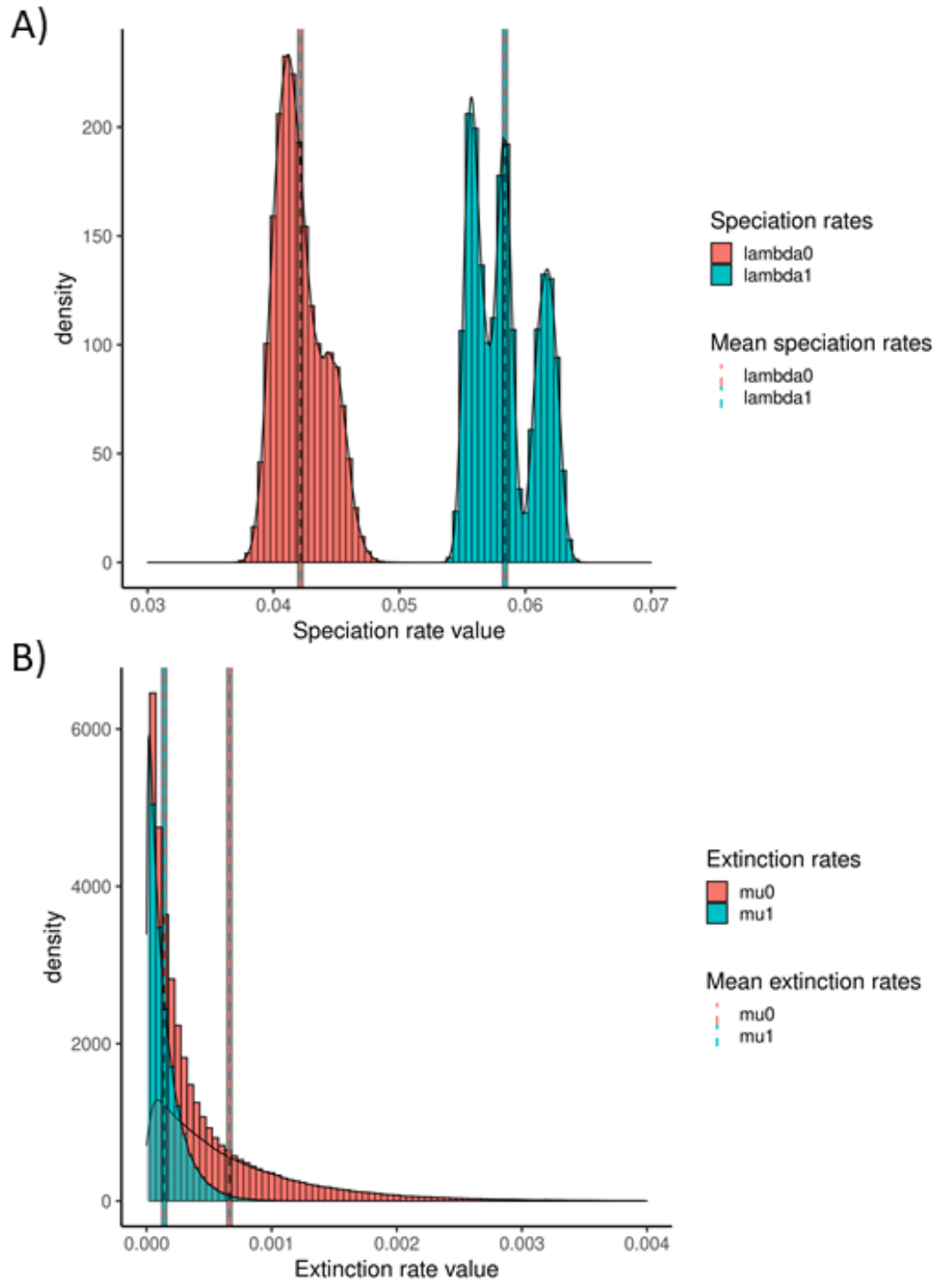

Figure 12. State dependent evolutionary rates of species with or without a cap. (A) Statedependent speciation rates. lambda0 $=$ speciation rate of lineages without a cap. lambdal $=$ speciation rate of lineages with cap. (B) State-dependent extinction rates. $m u 0=$ extinction rate of lineages without a cap. mul = extinction rate of lineages with cap

\subsubsection{Enclosed development}

The cap can give protection for the developing fruiting body by sheltering it from the environmental impacts above. However, many species produce fruiting bodies with extra protecting tissue layers and sheaths enveloping the spore-bearing structure (hymenophore) or the whole fruiting body. This motivated us to examine the macro- 
evolution of enclosed development in mushroom-forming fungi. We created three empirical character states (Table 2.). Based on both the BayesTraits and MuSSE analyses, we found that the transition rate from semi-enclosed development to enclosed development was 16.03 - 19.31 times higher than that in the reverse direction (q12 > q21), depending on the method. Moreover, the transition rate from semi-enclosed development to open development was also $3.75-25.14$ times higher than in the reverse direction (q10> q01), depending on the method (Figure 13. and Electronic Appendix 4). The high transition rates of q12 and q10 suggest that semi-enclosed development is a labile state and when it evolves, it often advances to enclosed development or reverses to open development. The forward and reverse transition rates between open and enclosed development were relatively small and did not differ significantly (Electronic Appendix 4). Based on model tests (reduced models with one of the rates constrained to 0 ), we found that all the six transition rates were a significant element of our model. Therefore, the evolution of enclosed development could go through the semi-enclosed state (mean rates, $\mathrm{q} 01=0.01$ and $\mathrm{q} 12=0.82$ ) or could directly evolve from open development (mean rate, $\mathrm{q} 02=0.03)$. 


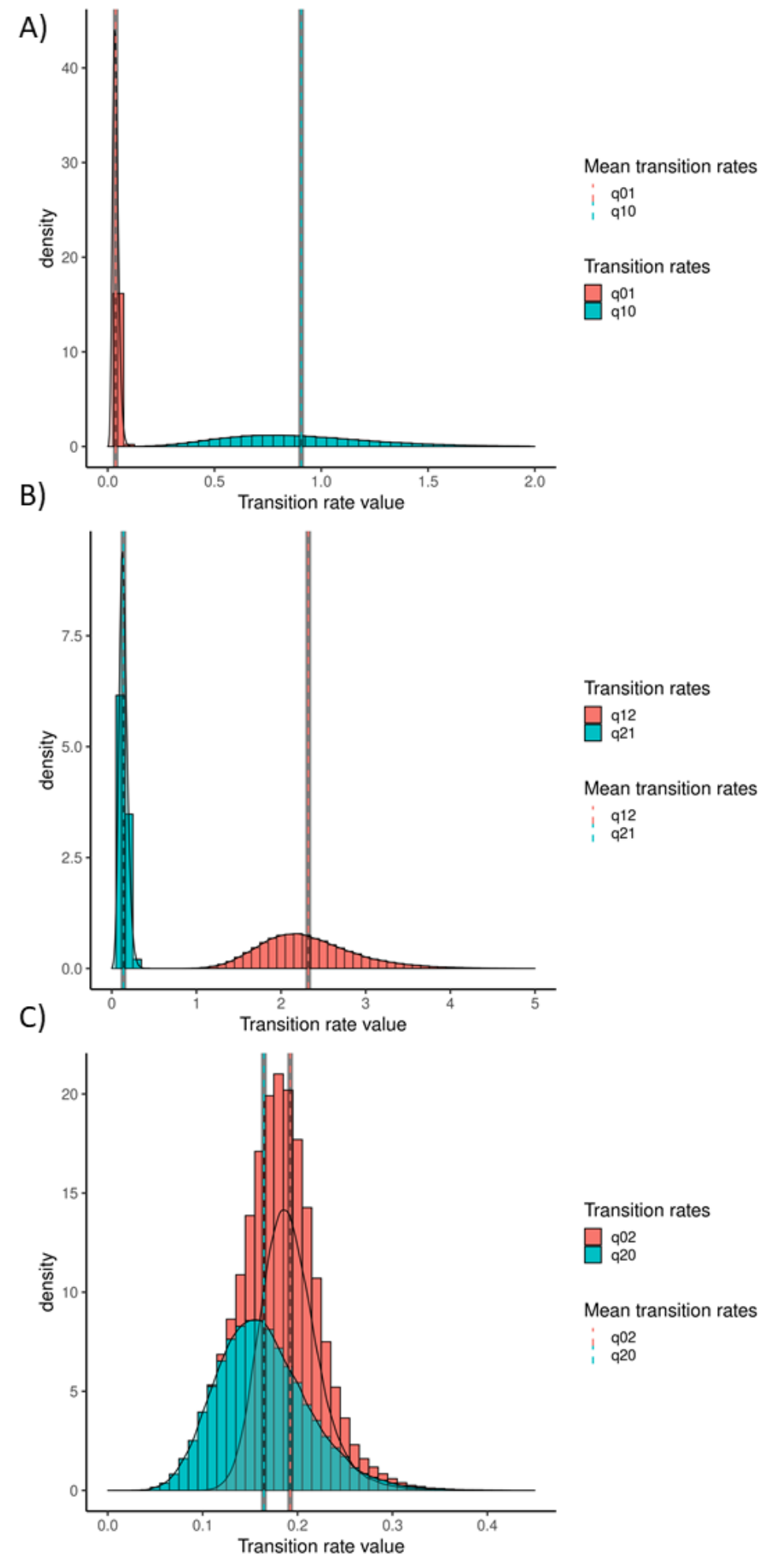

Figure 13. The distribution of the inferred transition rates of enclosed development trait using BayesTraits Bayesian MCMC analysis. (A) Transition rates between the open (state 0) and semienclosed (state 1) development states. (B) Transition rates between the semi-enclosed (state 1) and enclosed (state 2) development states. $(C)$ Transition rates between the open (state 0$)$ and enclosed (state 2) development states. qij represents the transition rate from state $i$ to state $j$. 
By analyzing trait dependent diversification rates, we revealed that species with semi-enclosed development had the highest diversification rate (mean rates, $6.99 \times 10^{-2}$ and $7.04 \times 10^{-2}$, Bayesian or ML analyses, respectively). This was followed by enclosed development (mean rates, $5.80 \times 10^{-2}$ and $5.81 \times 10^{-2}$, Bayesian or ML analyses, respectively) and open development (mean rates, $4.80 \times 10^{-2}$ and $4.81 \times 10^{-2}$, Bayesian or ML analyses, respectively). The differences in diversification rates were mainly driven by the speciation rate, because 26 likelihood ratio tests out of 30 showed non-significant differences between any pair of extinction rates, but speciation rates were different in all cases (LRT, p<0.05, Electronical Appendix 5).

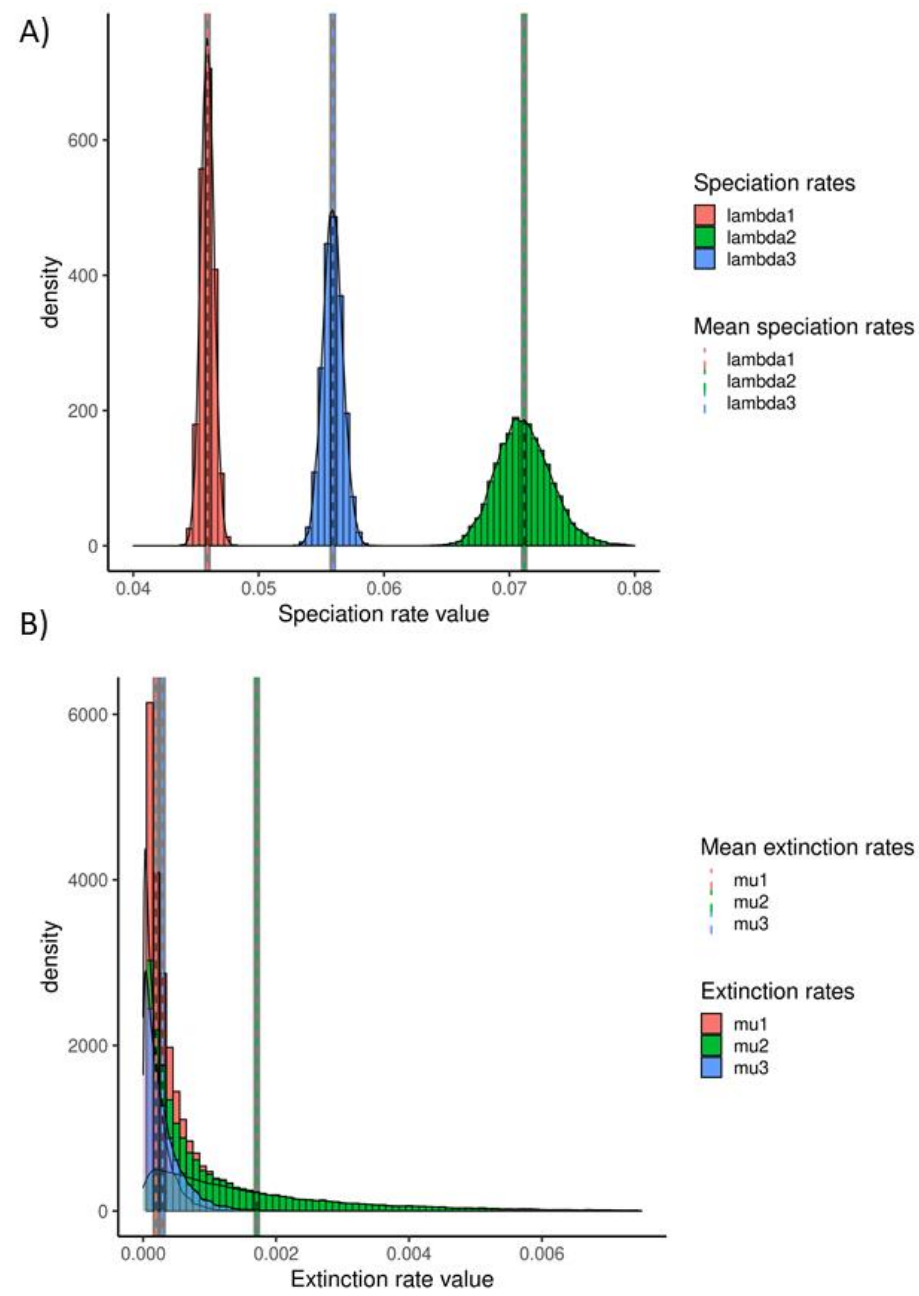

Figure 14. Results of the analysis of enclosed development trait using the MuSSE model. (A) State-dependent speciation rates. lambdal = speciation rate of lineages with open development. lambda $2=$ speciation rate of lineages with semi-enclosed development. lambda $3=$ speciation rate of lineages with enclosed development. (B) State-dependent extinction rates. mul = extinction rate of lineages with open development. $m u 2=$ extinction rate of lineages with semienclosed development. mu3 = extinction rate of lineages with enclosed development. 
A significant portion of species with enclosed or semi-enclosed characters produce protecting tissues which cover the whole fruiting body (universal veil) or only the hymenophore (partial veil). Therefore, we asked whether solely these traits could influence the evolution of mushroom-forming fungi. Interestingly the transition rate from universal or partial veil to non-veil states was $3.09-3.22$ or $10.69-11.20$ times higher than the reverse directions depending on the method, respectively (Figure 15., Electronic Appendix 4). This suggests that protecting veils were frequently lost through the evolution of mushroom-forming fungi.

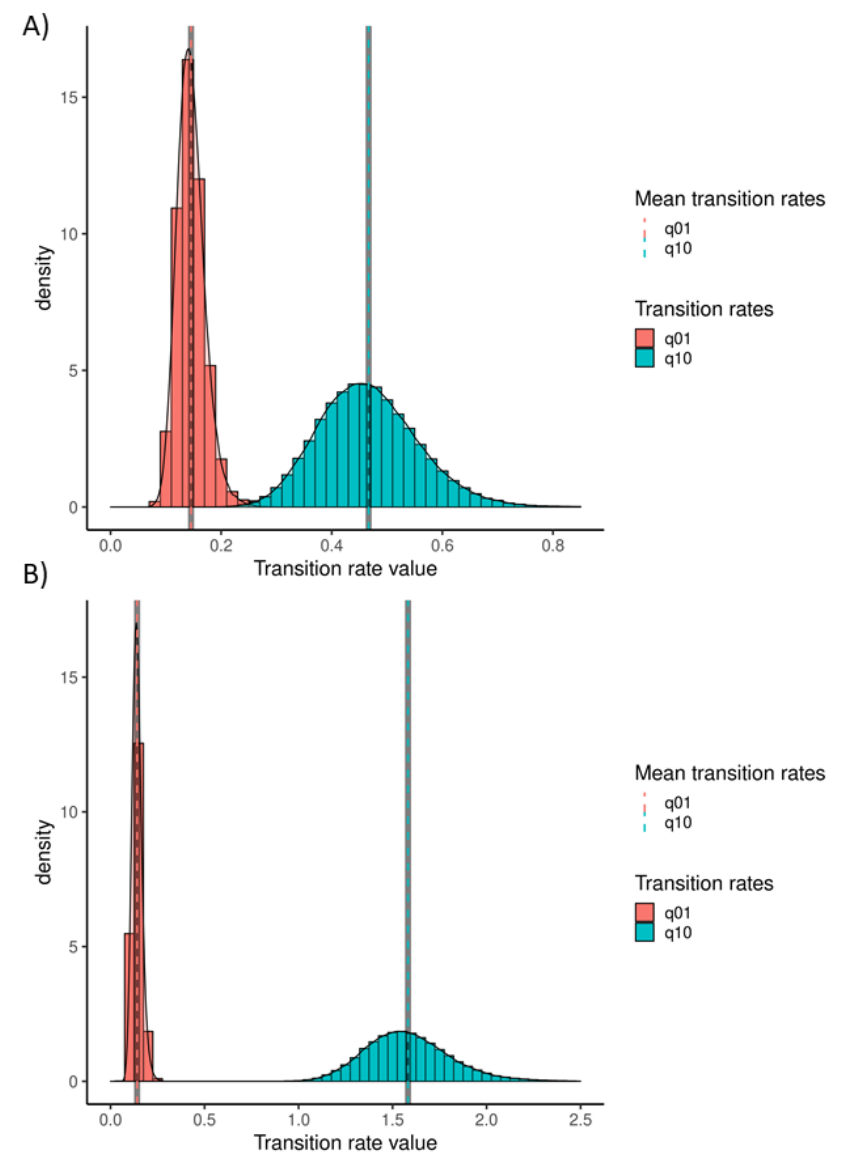

Figure 15. The posterior distribution of transition rates from non-veiled towards veiled forms and vice versa. (A) Universal veil, where q01 represents the transition rate from the non-veiled state towards the veiled state and the q10 represents the transition rate of the reverse directions. $(B)$ Partial veil, where q01 represents the transition rate from the non-veiled state towards the veiled state and the 110 represents the transition rate of the reverse directions.

The higher transition rates towards non-veiled states were also supported by likelihood ratio tests and Bayes factors performed on both universal and partial veil dataset (Electronic Appendix 4). However, the diversification rate of species with a universal or partial veil was 1.23 and 1.33 times higher than species without any of the veils (Figure 16.). This pattern of diversification rate was driven by speciation rate because no 
differences were found between the extinction rates of different states by analyzing any of the veil trait datasets. The higher speciation rate of species with protecting veils was supported by likelihood ratio tests, which proved to be significant in both universal and partial veils (Electronic appendix 5).
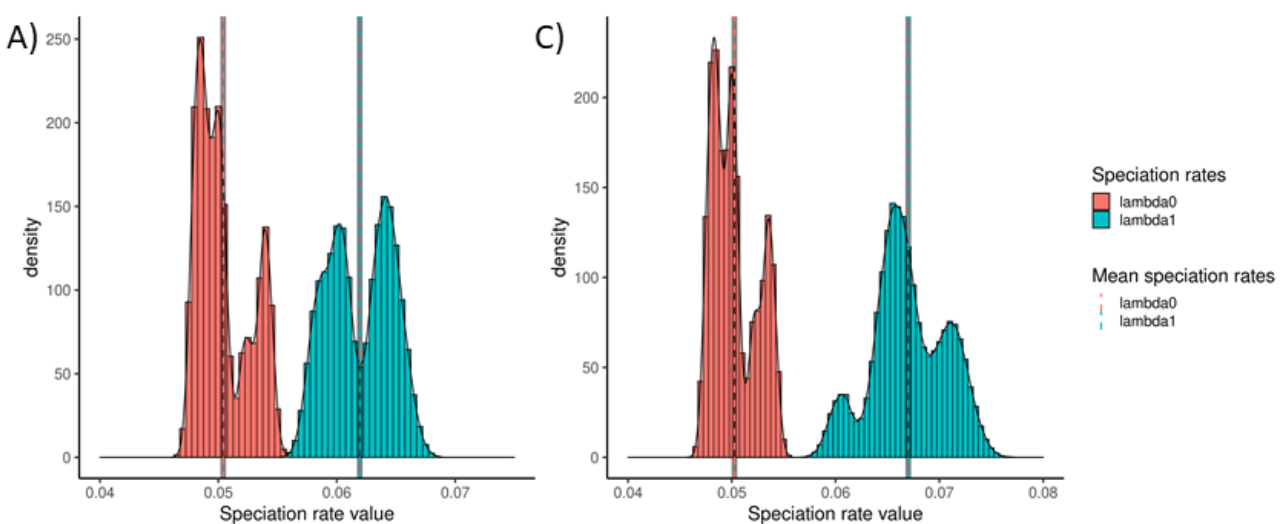

B)

D)
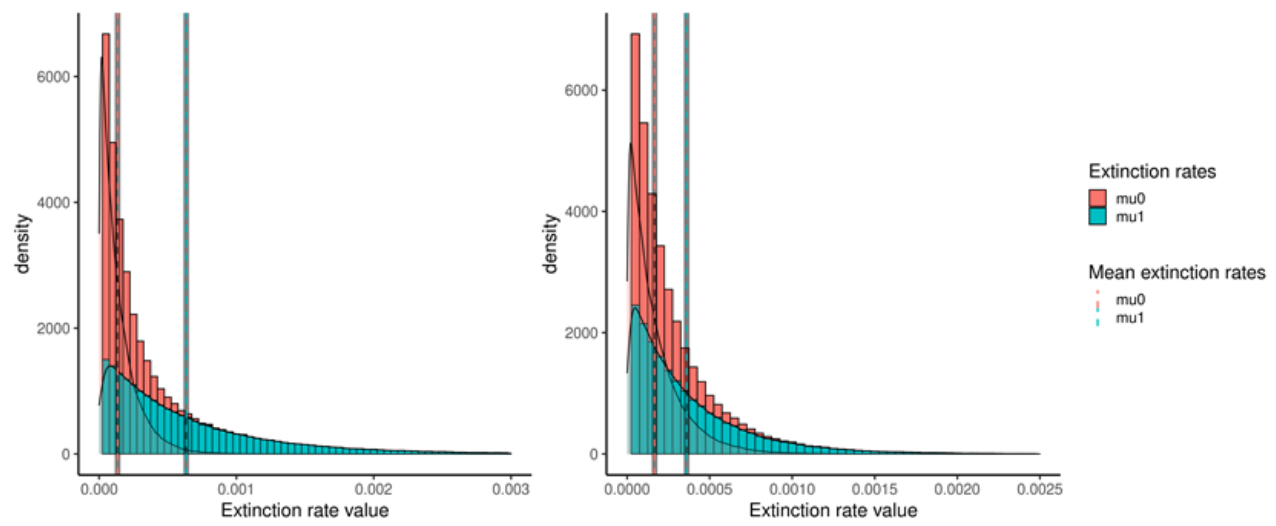

Figure 16. Results of the trait dependent speciation and extinction rate analyses of universal veil $(A-B)$ and partial veil (C-D). (A) Speciation rate of lineages without universal veil (lambda0) or with it (lambdal). (B) The extinction rate of lineages without universal veil (muO) or with it (mul). (C) Speciation rate of lineages without partial veil (lambda0) or with it (lambdal). (D) The extinction rate of lineages without partial veil (mu0) or with it (mul).

\subsubsection{Increased hymenophore surface area}

Meiospores are produced on the spore-bearing structure, called hymenophore. The simplest hymenophore expands on smooth surfaces of the fruiting body. By developing structured hymenophores (e.g., veins, spines, pores or lamellae), the number of spores produced per fruiting body mass can be increased. That is why we hypothesized that complex hymenophores could be evolutionarily advantageous for species. We defined three discrete character states corresponding with how structured the hymenophore is (Table 2.) and inferred trait evolution and trait dependent diversification rates. Both BayesTraits and MuSSE analyses showed that the transition rate from weakly structured 
hymenophore to well-developed hymenophore (q12) was 54.3 - 61.5 times higher (LR test, $\mathrm{p}<0.05, \log$ Bayes factor $>38$ ) than the reverse direction (q21). Weakly structured hymenophore could be a labile state, because the transition rate from it towards smooth hymenophore (q10) was 14.9 - 17.1 times higher (LR test, $p<0.05, \log$ Bayes factor $>$ 15.4) than in the reverse direction (Figure 17, Electronic Appendix 4).

A)

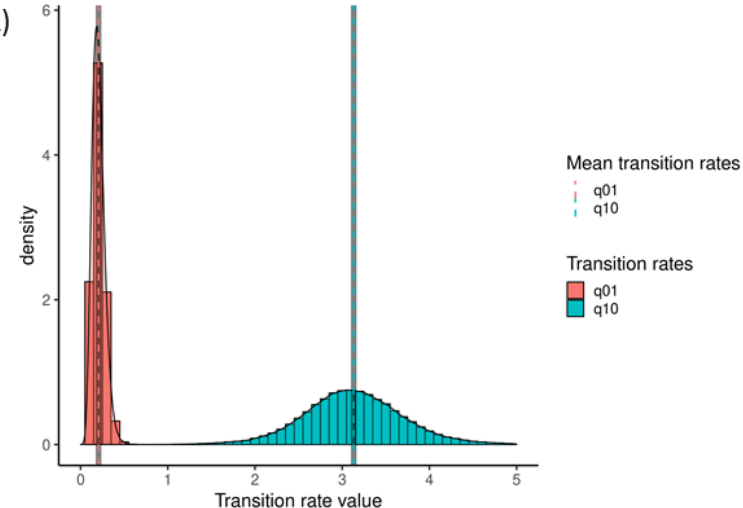

B)

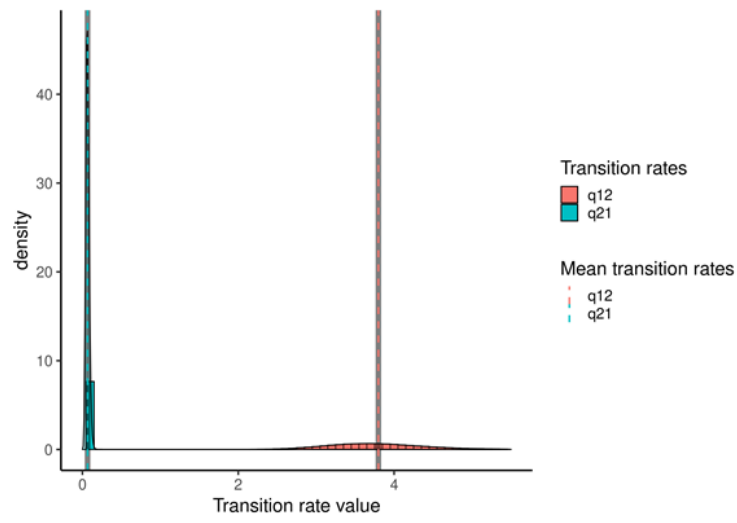

C)

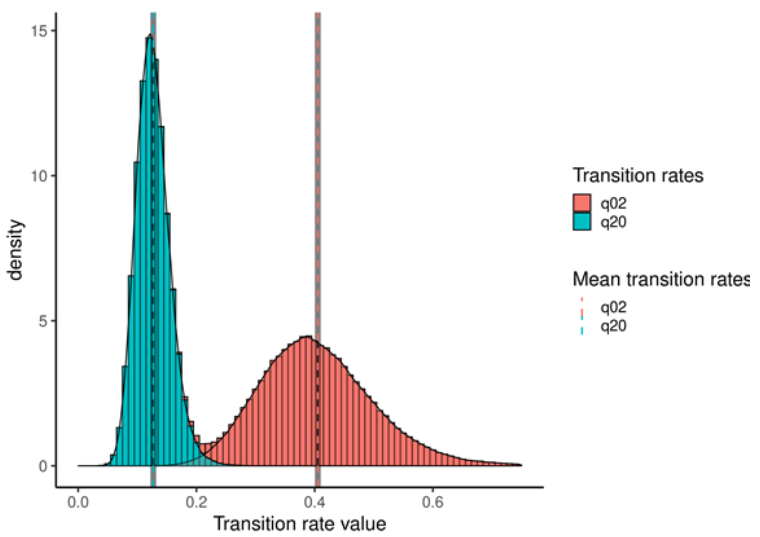

Figure 17. The posterior distribution of the inferred transition rates between three different hymenophore types. (A) Transition rates between smooth (state 0) and weakly structured (state 1) hymenophores. (B) Transition rates between weakly structured (state 1) and well-developed (state 2) hymenophores. (C) Transition rates between smooth (state 0) and well-developed (state 2) hymenophores. qij represents the transition rate from state $i$ to state $j$, e.g., q01 means the transition rate from state 0 to state 1 . 
The diversification rate of species with well-developed hymenophore was 1.36 and 1.41 times higher than that of species with weakly structured and smooth hymenophore, respectively (Figure 18.). The higher diversification rate associated with a well-developed hymenophore was also supported by a likelihood ratio test $(\mathrm{p}<0.05)$. Interestingly, the diversification rate of smooth hymenophore and weakly structured hymenophore was similar (4.05 x $10^{-2}$ and $\left.4.18 \times 10^{-2}\right)$ and no differences were revealed by likelihood ratio tests $(\mathrm{p}<0.05$, Electronic Appendix 5). The differences in diversification rates were mainly driven by the speciation rate, because we found nonsignificant differences between any pair of extinction rates based on likelihood ratio test $(\mathrm{p}<0.05)$.

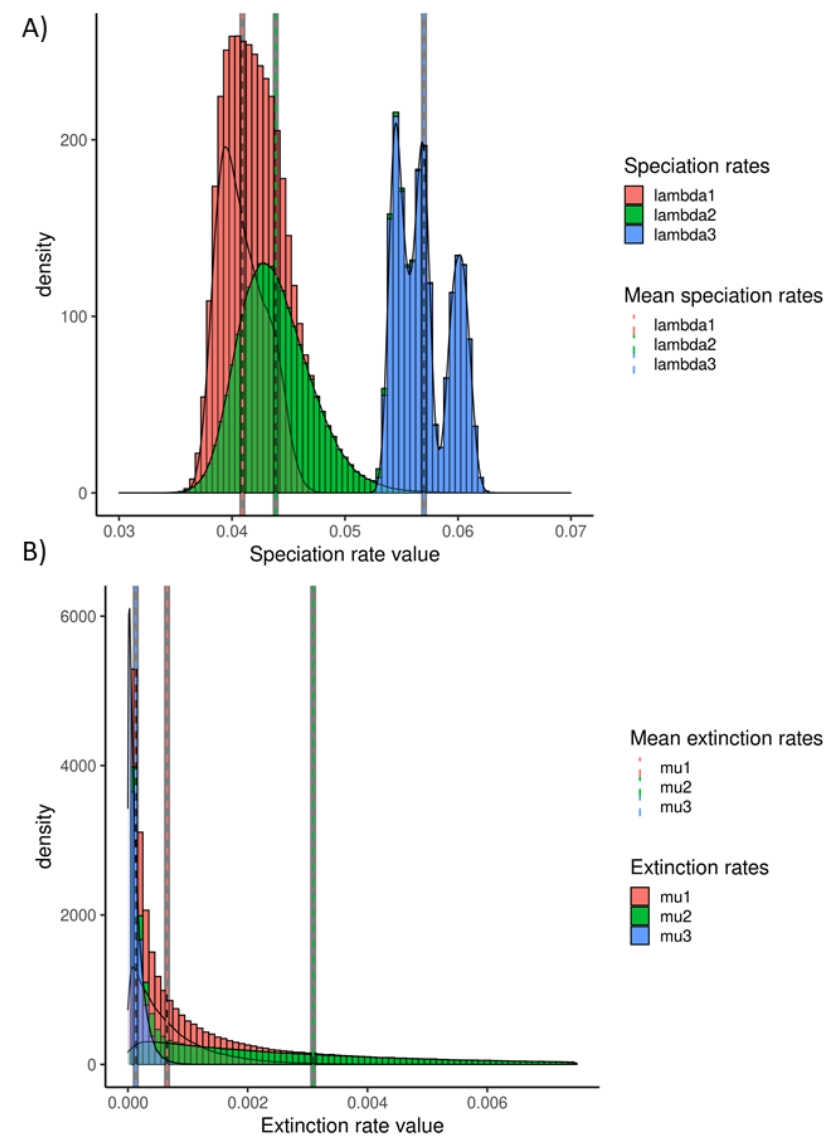

Figure 18. Trait dependent evolutionary rates estimated under MuSSE model for enclosed development trait. (A) State-dependent speciation rates. lambdal $=$ speciation rate of lineages with open development. lambda $2=$ speciation rate of lineages with semi-enclosed development . lambda3 = speciation rate of lineages with enclosed development. (B) State-dependent extinction rates. $m u 1=$ extinction rate of lineages with open development. $m u 2=$ extinction rate of lineages with semi-enclosed development. mu3 = extinction rate of lineages with enclosed development. 


\subsection{Tissue-specific low-input RNA-seq}

\subsubsection{General description of the low-input RNA-seq workflow}

In paragraph 4.1, we found that morphological traits such as cap, protecting sheaths (universal or partial veil), or the increased hymenophore surface area could positively affect the diversification of mushroom-forming fungi. To uncover genes that could have a significant role in the development of structures examined through the macro-evolutionary analyses, we developed a low-input RNA-seq procedure coupled with laser capture microdissection (LCM). We examined the early developmental stages of Coprinopsis cinerea A43mutB43mut and focused on the cap, the universal veil, the partial veil and the most structured hymenophore, the lamella. The overview of the workflow can be seen on Figure 19. We performed a number of adjustments and modifications of existing methods, which is described in the following paragraphs. The detailed protocol can be found in Appendix 1.

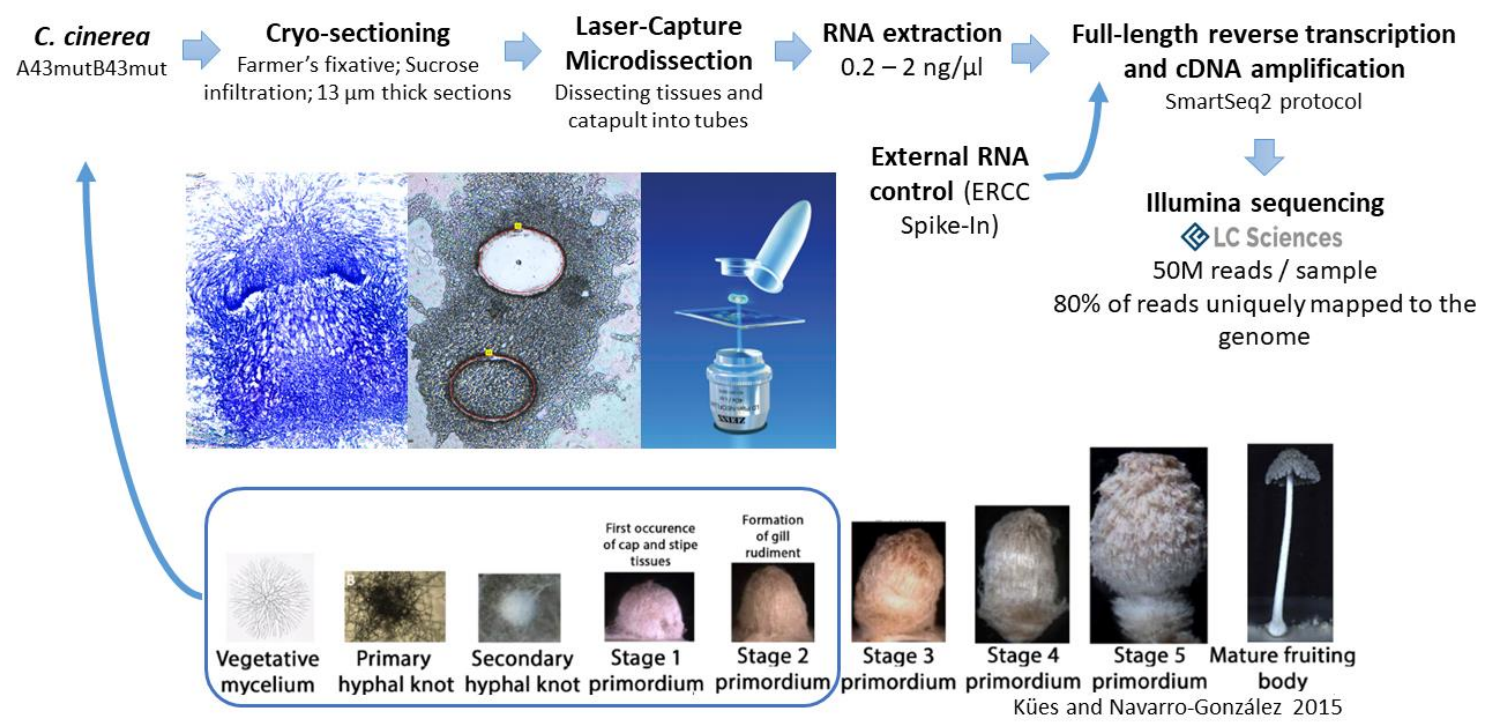

Figure 19. Main steps of the low-input tissue-specific RNA sequencing workflow

\subsubsection{Modifications and adjustments on the RNA-seq workflow}

\subsubsection{RNA protecting agents}

We tested the effect of two RNA protecting agents, 2-mercaptoethanol and QIAGEN RNAlater RNA stabilization reagent on bulk RNA extracts and found no considerable influence on the RNA quality. We measured a similar amount of RNA concentrations $(0.06-0.17 \mathrm{ng} / \mu \mathrm{l} \times \mathrm{mg}$ of fresh primordium $)$ in all the four treatments (including controls), but as was expected, the positive control (extraction from fresh 
primordium) contained RNA with the highest concentration. We did also not see considerable differences on the agarose gel electropherogram (Figure 20.), therefore we decided to apply 2-mercaptoethanol through our protocol because, in smaller dimensions (below bulk RNA-seq), the role of RNA protective agents could become more critical, and 2-mercaptoethanol is more affordable than QIAGEN RNAlater RNA stabilization reagent.

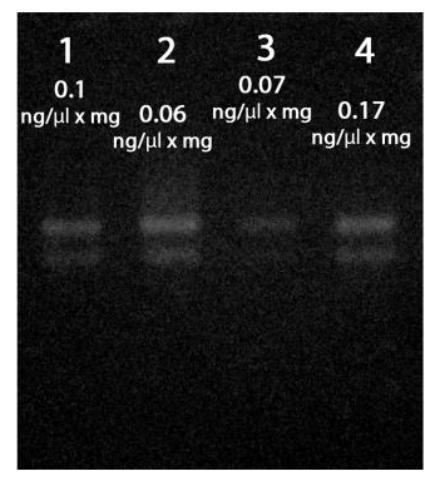

Figure 20. Testing the effect of different RNA protecting agents. Fixation, infiltration and embedding were applied on stage 3 primordium. 1. No treatment. 2. QIAGEN RNAlater RNA stabilization reagent, 3. 2-mercaptoethanol and 4. control (extraction from fresh primordium)

\subsubsection{Cryo-sectioning}

We found that $-20^{\circ} \mathrm{C}$ was the optimal cutting temperature for fruiting body tissues embedded in cryomount. $13 \mu \mathrm{m}$ thick sections were easy to handle through the entire workflow but were thin enough to recognize main tissue types under a bright field microscope. Thinner sections were easily damaged and torn during microdissection. We also found that natural adhesion of tissues to the glass slide or the PEN covered slide was not strong enough. Therefore we applied a poly-L-lysine layer on the PEN membrane glass slide, which supplied sufficient adhesion for tissues. At the same time, tissues were easily detached from the slide through the LCM procedure due to the PEN membrane.

\subsubsection{Cell disruption}

We tested the digesting capability of a chitinase (Sigma-Aldrich) and an enzyme mixture (1.5\% snail enzyme extract and $0.5 \%$ chitinase) on mycelium samples. We found that the chitinase had a weak effect on mycelium integrity, and only a few hyphal tips showed a swollen morphology after 30 minutes. Most of the hyphae were also intact after applying the enzyme mixture and did not show a better digesting attribute then the pure chitinase. 


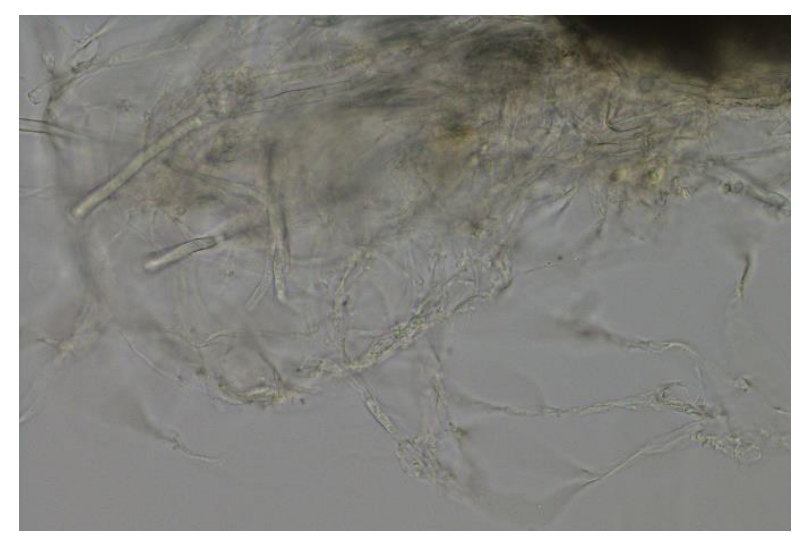

Figure 21. Intact hyphae after 30 minutes digestion of mycelium with the enzyme mixture $(1.5 \%$ snail enzyme extract and $0.5 \%$ chitinase). $200 x$ magnification.

Furthermore, we wanted to check whether the enzyme solutions (chitinase or enzyme mixture) could affect pure RNA extract. Based on visual inspection of electropherograms, we found that RNA was slightly (Figure 22.) or fully degraded (data not shown) when chitinase or enzyme mixture was applied, respectively. The full degradation of RNA when the enzyme mixture was used can be explained by the superior purity of the vendor's product over that of the snail enzyme extract.

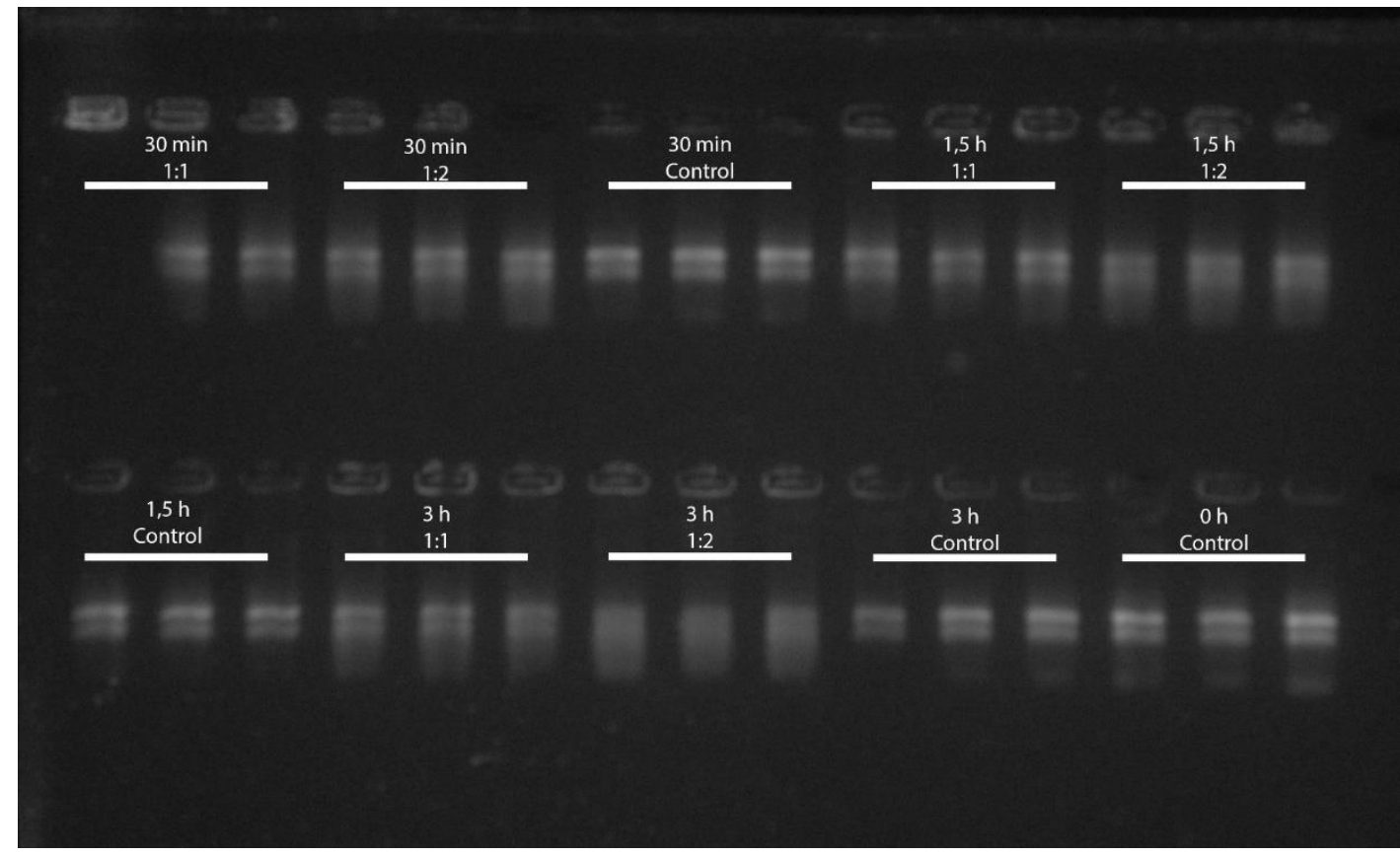

Figure 22. The result of testing the effect of chitinase on RNA extract. The two pronounced bands represent $28 S$ (upper) and $18 S$ (lower) rRNA. Note that the bands became blurred with time and enzyme ratio (1:1 or 1:2 v/v\% of the RNA extract and enzyme solution). 
We further investigated the applicability of chitinase in the workflow by catapulting tissue samples into $10 \mu \mathrm{l}$ chitinase using LCM. $20 \mu 1$ extraction buffer $\left(\right.$ PicoPure $^{\mathrm{TM}}$ ) was added to the samples after 30 minutes-long incubation at $42^{\circ} \mathrm{C}$. Then we proceeded with the default protocol starting with the serial heat shock treatment (Appendix 1). Unfortunately, the application of chitinase did not yield an improvement because the yield was less than in the control sample $(\sim 500 \mathrm{pg} / \mu \mathrm{l}$ versus $\sim 3000 \mathrm{pg} / \mu \mathrm{l})$ and the measured RNA integrity was also low (Figure 23.).
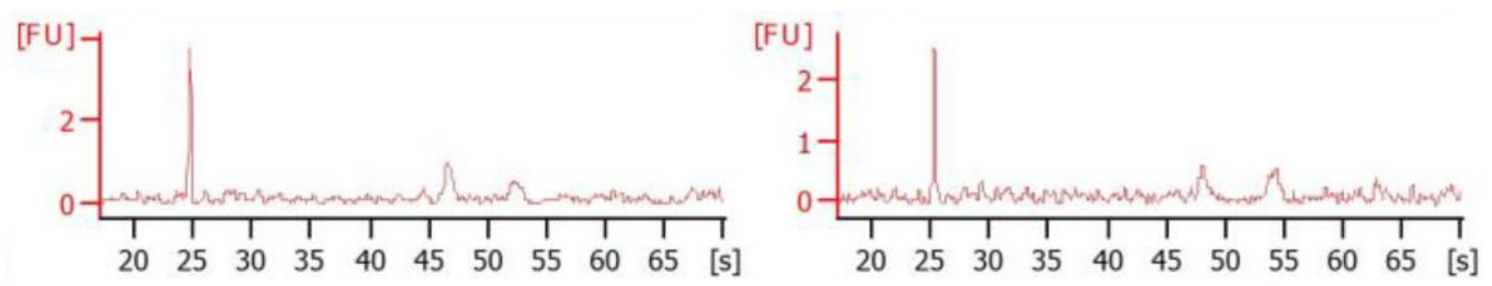

Figure 23. Electropherograms produced by using Bioanalyzer Instrument. Both analyses based on RNA extractions using chitinase at the cell disruption step. In an ideal case, two sharp peaks should be displayed at around 45 and 50 sec migration time which corresponds to 2000 and 3000 nucleotide length of the $18 S$ and $28 S$ rRNA respectively. Left panel: RNA concentration $\approx$ $780 \mathrm{pg} / \mu \mathrm{l}, \mathrm{RIN}=4.9$. Right panel: $R N A$ concentration $\approx 590 \mathrm{pg} / \mu \mathrm{l}, \mathrm{RIN}=4.4 . R I N=R N A$ integrity number.

We also tested whether we could substitute the extraction buffer of the PicoPure ${ }^{\mathrm{TM}}$ RNA isolation kit, which might improve the cell wall disruption. We found that no RNA was obtained by using the extraction buffer of the NucleoSpin ${ }^{\mathrm{TM}}$ kit at the cell disruption step (Figure 24.), therefore it was not applicable in the workflow.
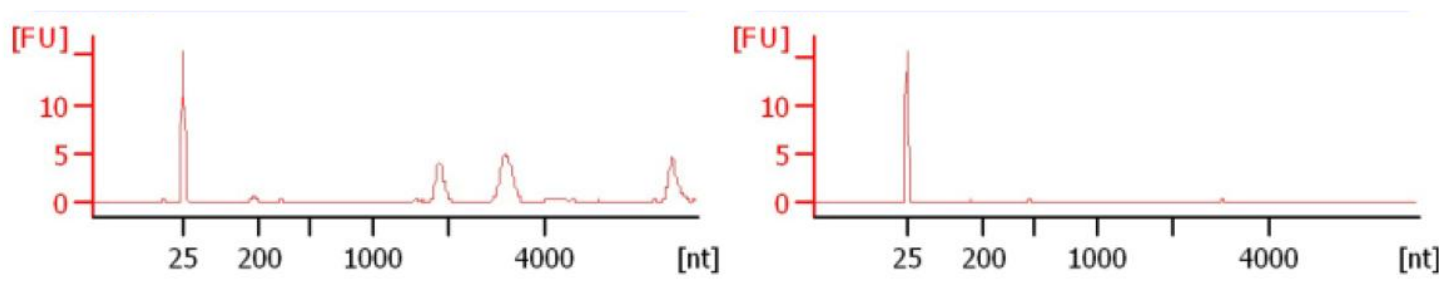

Figure 24. Electropherograms produced by using Bioanalyzer Instrument. In an ideal case, two sharp peaks should be displayed at around 45 and 50 sec migration time which corresponds to $\sim 2000$ and 3000 nucleotide length of the 18S and 28S rRNA respectively. Left panel represents the sample where the extraction buffer of the PicoPure ${ }^{T M}$ RNA kit was used (RIN =9.3, RNA concentration $\approx 230 \mathrm{pg} / \mu \mathrm{l}$ ). Right panel shows the sample where the extraction buffer of NucleoSpin ${ }^{T M}$ RNA kit was used $(R I N=N / A, R N A$ concentration $\approx 0 \mathrm{pg} / \mu \mathrm{l}) . R I N=R N A$ integrity number. 
Based on the above-mentioned experiments, we only applied a heat shock treatment of the samples in the extraction buffer of the PicoPure ${ }^{\mathrm{TM}}$ RNA isolation kit through the final protocol (Appendix 1).

\subsubsection{Tissue sampling and RNA-seq}

We performed preliminary histological investigations to thoroughly scrutinize main tissue types through the early development of $C$. cinerea (Figure 25.). By examining more than 200 specimens, we detected intermediates between the secondary hyphal knot and stage 1 primordium. We followed Kües \& Navarro-González, 2015 to define developmental stages but we examined other works as well through the descriptions of new intermediate stages (Matthews and Niederpruem, 1973; Moore, 1995; Kües, 2000; Clémencon, 2012).

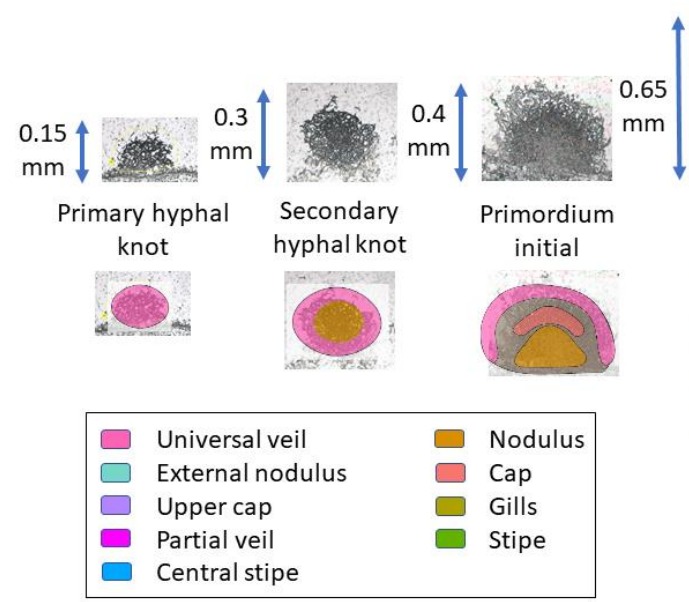

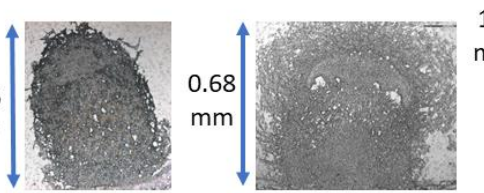
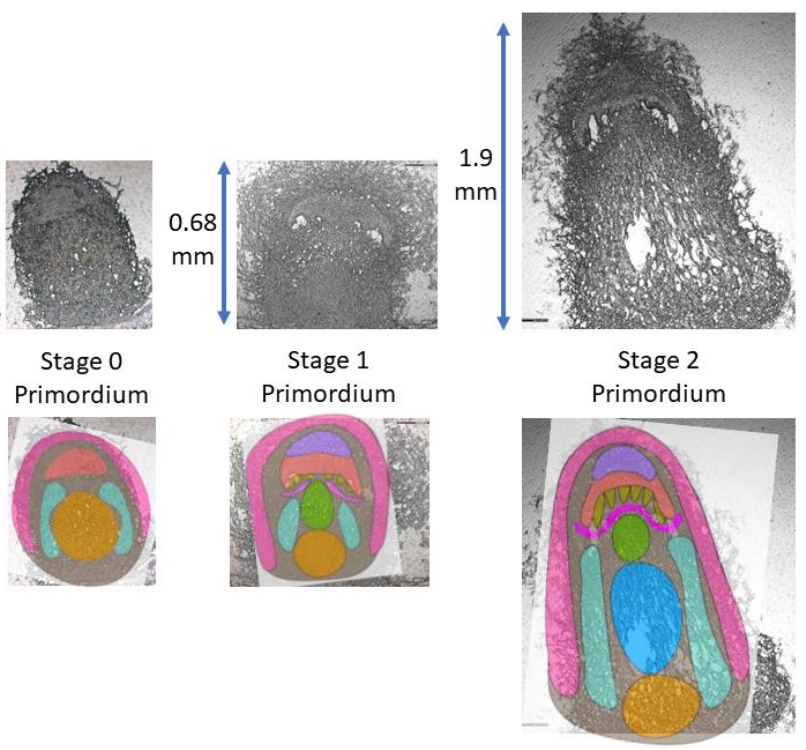

Figure 25. Seven developmental stages (vegetative mycelium is not shown here) and nine tissue types were examined through the early development of $C$. cinerea. Primordium initial and stage 0 primordium are first described in detail in this study.

We observed a stage (here it is called primordium initial), which presumably follows the development of the secondary hyphal knot. The primordium initial had a similar size $(\sim 0.3 \mathrm{~mm})$ to the secondary hyphal knot, however, the nodulus was oval shaped, and it elongated towards the base of the primordium (Figure 26.). Contrarily, in a secondary hyphal knot the nodulus is surrounded by the universal veil, and it has a round shape (Figure 27.). At the top of the nodulus of the primordium initial, a denser tissue layer can be recognized, which we think is the initial of the cap tissue. Moreover, in some cases, primordia with a minute pinhead at the nodulus could be seen (Figure 28.). This 
pinhead (cap initial) consisted of narrow hyphae densely arranged similarly to the structure of the nodulus. The primordium initial did not show the characteristics of a primordium (e.g., well differentiated cap stipe and nodulus), but clearly differed from the secondary hyphal knot and exhibited the signs of tissue differentiation, therefore we consider it as a separate stage.
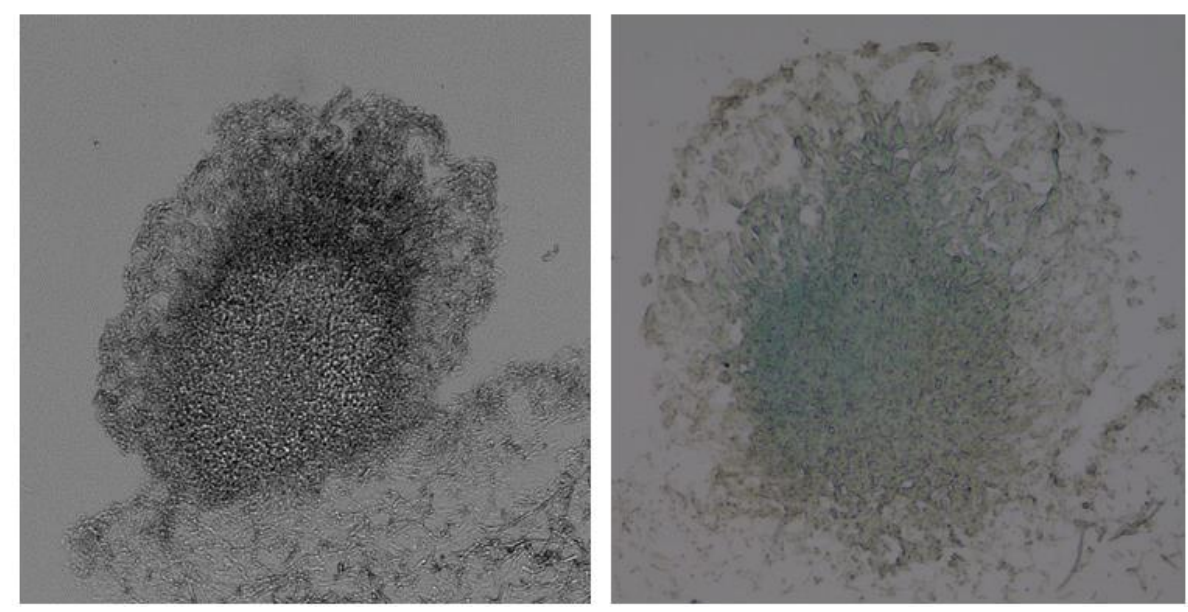

Figure 26. Examples for the primordium initial developmental stage. 100x magnification.

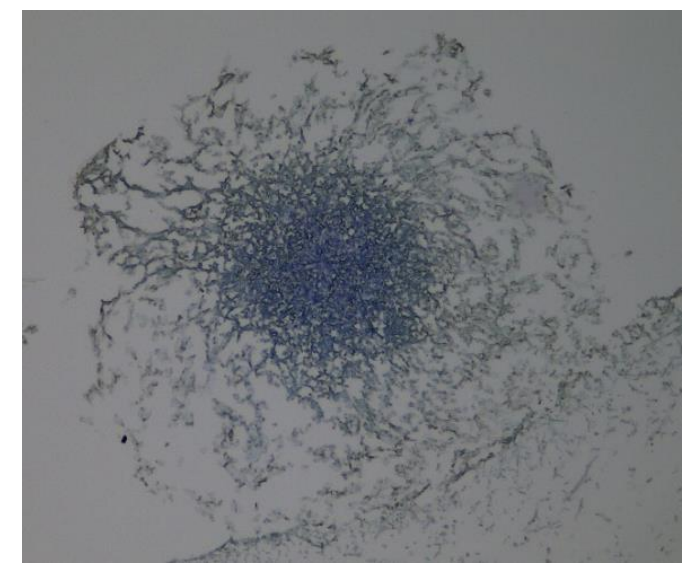

Figure 27. A typical secondary hyphal knot. The section was stained with MBA. 100x magnification. 

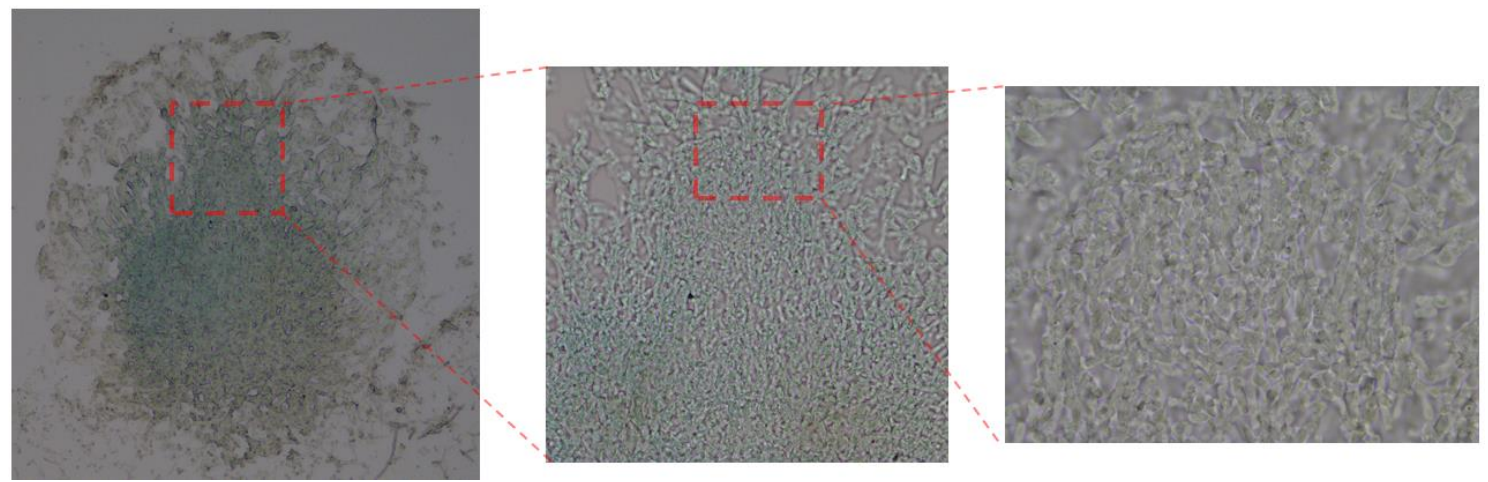

Figure 28. The structure of the cap initial in the primordium initial stage. 100x, 400x and 1000x magnification on the 1st, 2 nd and the 3rd images, respectively.

We observed another developmental stage before the stage 1 primordium, which we refer to as stage 0 primordium (Figure 29.). Stage 0 primordium clearly shows the outline of the cap, but this cap tissue is round or oval-shaped and not flattened as in later stages. Moreover, in stage 0 primordium the stipe tissue is not pronounced, in contrast to stage 1 primordium where the stipe is clearly differentiated from the surrounding tissues (Figure 30.).
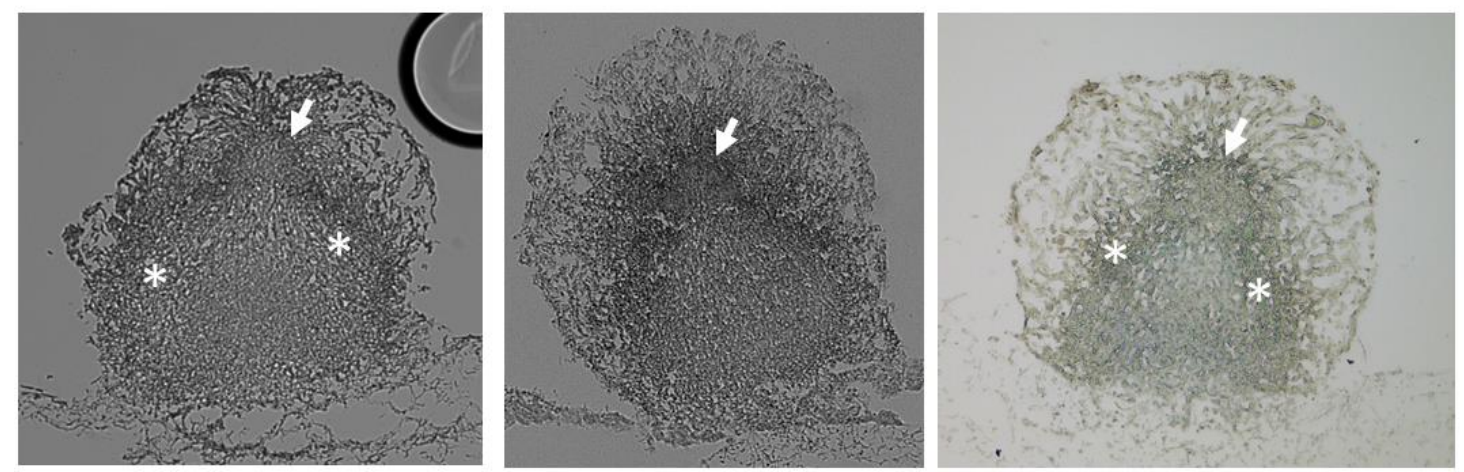

Figure 29. Examples for the stage 0 primordium. Cap (arrow) is visible, but still have a circular shape. External nodulus (asterisk) formed by loose hyphae between the nodulus and the universal veil. 100x magnification.

Starting from stage 0 primordium a loose structure develops around the nodulus, which is clearly not the part of the universal veil. To better disentangle tissue differentiation, we included this tissue type in our analyses, referring to it as 'external nodulus' (Figure 29. and Figure 30.).

Similarly, to the external nodulus, a loosely structured tissue could be recognized at the top of the cap in stage 1 primordium and stage 2 primordium. In this region, the cap tissue may continuously and gradually change into universal veil. It is possible that this tissue is not functionally separate from either the cap or the universal veil, but to increase 
resolution in our analyses, we treated this region as an independent tissue called 'upper cap'.

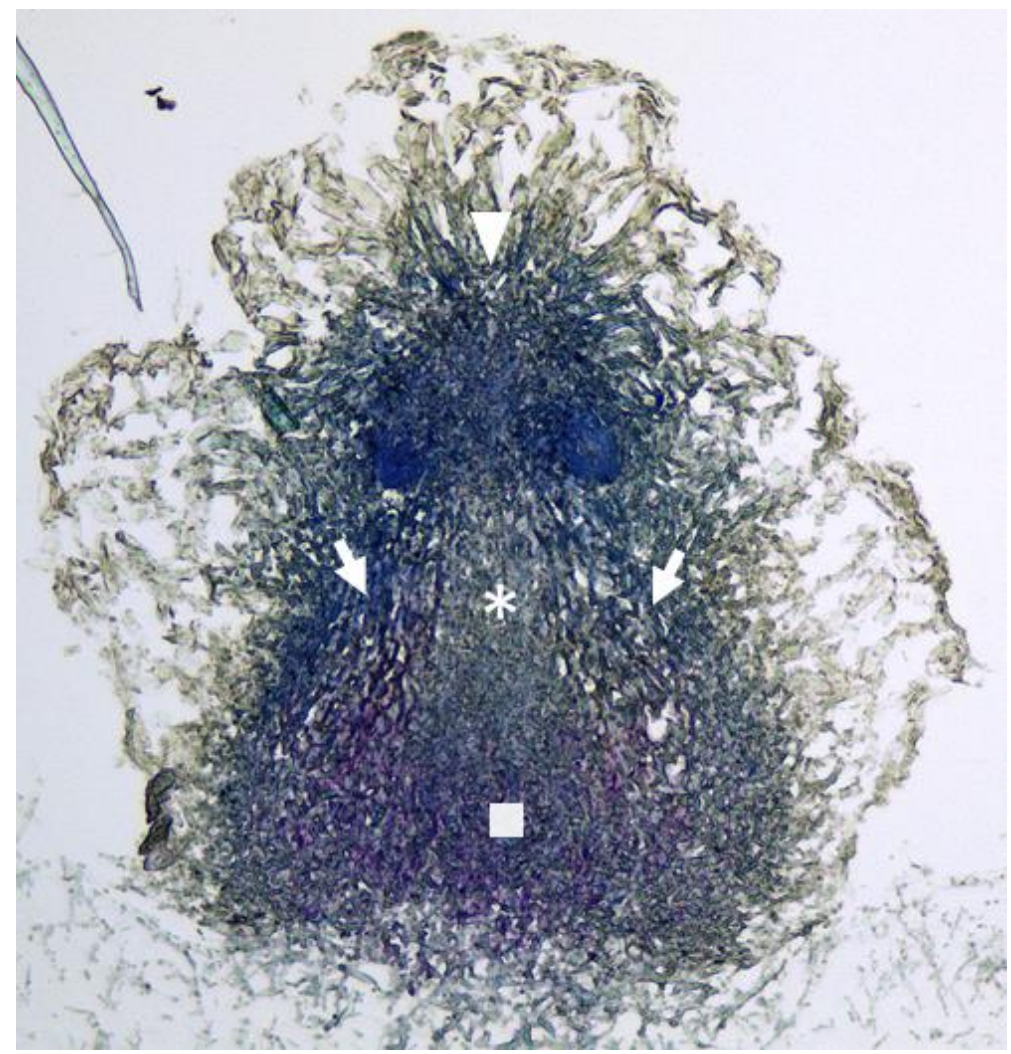

Figure 30. A typical stage 1 primordium. The stipe (asterisk) is clearly visible which spreads between the nodulus (rectangle) and the cap. Loose hyphae compose the external nodulus (arrows) around the nodulus and the stipe. Similarly, loose hyphae can be noticed at the top of the cap, named here upper cap (tip of the triangle). The section was stained with MBA. 100x magnification.

At stage 2 primordium, a slight expansion of the stipe can be noticed (Figure 25.) therefore, we additionally defined a 'central stipe' region. The central stipe can be found above the nodulus and consists of extremely loosely arranged hyphae, which went through an elongation process and has a loose fistulose structure. Probably, that is why we frequently experienced the damage of this tissue type during cryo-sectioning. The stipe of primordium stage 2 can be found between the central stipe and the cap related tissues (cap, lamellae, partial veil, Figure 25.). This tissue exhibited a denser and more robust structure than the central stipe. We considered hyphae, which spread below the lamellae from the edge of the cap to the stipe tissues to be the part of the partial veil. 
By screening primordia through 60 hours during our preliminary investigation, we did not find a connection between the time of the day and the occurrence of different developmental stages (Table 8.). Therefore, we sampled tissues in a four-hour window after the end of the dark period.

Table 8. The number of specimens found through different time points of the day. Sampling started from the $3^{\text {rd }}$ day of the day/light period of the culture and at every time point, five embedded materials were examined. Note that in some cases, we did not succeed in examining all materials due to tissue section preparation failure. In other cases, more than one primordium could be inspected in one material.

\begin{tabular}{|l|l|l|l|l|l|l|l|l|l|}
\hline & \multicolumn{7}{|c|}{ Time points and the \# of specimens } \\
\hline Stages & $14: 00$ & $24: 00$ & $3: 00$ & $7: 00$ & $17: 00$ & $21: 00$ & $2: 00$ & $8: 00$ & $14: 00$ \\
\hline Secondary hyphal knot & 2 & 2 & 1 & 2 & 3 & 3 & 1 & 1 & \\
\hline Primordium initial & & 2 & & 1 & & 1 & 1 & 2 & 2 \\
\hline Stage 0 primordium & & 1 & 1 & & 1 & & 1 & 1 & 1 \\
\hline Stage 1 primordium & 1 & 1 & 4 & 3 & 3 & 1 & 2 & 1 & 2 \\
\hline
\end{tabular}

Finally, nine tissue types and seven developmental stages were selected to be involved in our RNA-seq study, starting from the vegetative mycelium to primordium stage 2 (Figure 25.). From each tissue type, we isolated 28 sections of $\sim 584 \mu \mathrm{m}^{2}$ in area, resulting in, on average, $1.16+/-1.72 \mathrm{~mm}^{2}$ total area per tissue type per replicate (Table 9.). $2.3+/-2.6 \mathrm{ng} / \mu \mathrm{l}$ total RNA was extracted on average from a tissue sample and samples had sufficient quality for sequencing (RIN: $7.3+/-0.6$ ).

We aimed to obtain overall 27 sample types with four replicates meaning 108 RNA samples. However, in this study we worked on a preliminary dataset (87 samples) and in the future we will complement this with newly produced RNA-seq samples. Out of the 87 samples three did not pass the quality check after the reverse transcription and cDNA amplification therefore, sequencing was performed on 84 samples (Table 9.). 
Table 9. The 87 samples which were sent for sequencing. In the 'Sample ID' and the '\# of reads produced' columns NA represents samples that did not pass the quality check before sequencing. * shows samples that were excluded from our final analyses through the data quality check procedure (see below). ${ }^{a}$ represents a vegetative mycelium (VM) sample, which was retrieved from a section prepared for harvesting H1. Thus, a slightly different method was used here than in the case of other VM samples (see Materials and Methods). The "Days in light" column depicts the day on which the primordium was sampled. "Av. height" and "Av. width" give the average size of the primordia measured on the sections at approximately the mid-sagittal plane. "\# of regions catapulted" denotes the number of tissue regions which catapulted and pooled into a single tube. "Sum area" represents the total area of the catapulted regions. RIN $=R N A$ integrity number, $H 1=$ primary hyphal knot, $H 2=$ secondary hyphal knot, $P I=$ primordium initial, $P O=$ stage 0 primordium, $P 1=$ stage 1 primordium, $P 2=$ stage 2 primordium, $V M=$ vegetative mycelium, $U V=$ universal veil, $P V=$ partial veil, $E$. nodulus $=$ External nodulus, $C$. stipe $=$ Central stipe.

\begin{tabular}{|c|c|c|c|c|c|c|c|c|c|c|}
\hline $\begin{array}{c}\text { Sample } \\
\text { ID }\end{array}$ & $\begin{array}{c}\text { Days } \\
\text { in light }\end{array}$ & $\begin{array}{l}\text { Av. height } \\
(\mu \mathrm{m})\end{array}$ & $\begin{array}{l}\text { Av. width } \\
(\mu \mathrm{m})\end{array}$ & $\begin{array}{c}\text { Sum area } \\
(\mathrm{mm} 2)\end{array}$ & $\begin{array}{c}\text { \# of regions } \\
\text { catapulted }\end{array}$ & RIN & $\begin{array}{l}\text { Concentra- } \\
\text { tion }(\mathrm{pg} / \mu \mathrm{l})\end{array}$ & Stage & Tissue & $\begin{array}{r}\# \text { of reads } \\
\text { produced } \\
\end{array}$ \\
\hline 58 & 1 & - & - & 10.38 & 71 & 6.4 & 5,393 & VM & $\mathrm{VM}$ & $4.16 \mathrm{E}+07$ \\
\hline 59 & 1 & - & - & 8.07 & 57 & 7.1 & 3,531 & VM & VM & $5.00 \mathrm{E}+07$ \\
\hline $92^{\mathrm{a}}$ & 3 & - & - & 0.77 & 12 & 7.4 & 148 & VM & VM & $4.70 \mathrm{E}+07$ \\
\hline 130 & 2 & - & - & 5.42 & 31 & 6.8 & 7,445 & VM & VM & $4.81 \mathrm{E}+07$ \\
\hline 3 & 3 & 157 & 216 & 0.71 & 19 & 7.0 & 621 & $\mathrm{H} 1$ & Nodulus & $5.04 \mathrm{E}+07$ \\
\hline 88 & 3 & 152 & 346 & 0.77 & 12 & 7.8 & 294 & H1 & Nodulus & $5.50 \mathrm{E}+07$ \\
\hline $103^{*}$ & 3 & 296 & 396 & 1.79 & 18 & 7.5 & 3,189 & H1 & Nodulus & $5.56 \mathrm{E}+07$ \\
\hline NA & 3 & 150 & 398 & 0.49 & 8 & 7.1 & 1,279 & H1 & Nodulus & NA \\
\hline $104 *$ & 3 & 143 & 278 & 0.26 & 5 & 7.3 & 143 & $\mathrm{H} 1$ & Nodulus & $4.70 \mathrm{E}+07$ \\
\hline 60 & 4 & 381 & 496 & 0.14 & 7 & 6.9 & 338 & $\mathrm{H} 2$ & Nodulus & $4.62 \mathrm{E}+07$ \\
\hline 84 & 5 & 486 & 560 & 0.63 & 14 & 7.6 & 1,156 & $\mathrm{H} 2$ & Nodulus & $5.39 \mathrm{E}+07$ \\
\hline 89 & 4 & 520 & 687 & 0.76 & 12 & 8.9 & 7,361 & $\mathrm{H} 2$ & Nodulus & $5.05 \mathrm{E}+07$ \\
\hline $93 *$ & 3 & 270 & 356 & 0.07 & 7 & 7.3 & 955 & $\mathrm{H} 2$ & Nodulus & $4.21 \mathrm{E}+07$ \\
\hline 11 & 4 & 520 & 687 & 0.76 & 12 & 7.9 & 11,589 & $\mathrm{H} 2$ & UV & 4.17E+07 \\
\hline 94 & 3 & 270 & 356 & 0.07 & 7 & 7.6 & 5,778 & $\mathrm{H} 2$ & UV & $5.21 \mathrm{E}+07$ \\
\hline $95 *$ & 4 & 116 & 220 & 0.98 & 25 & 7.6 & 1,111 & $\mathrm{H} 2$ & UV & $5.23 \mathrm{E}+07$ \\
\hline 105 & 3 & 217 & 352 & 1.00 & 29 & 7.5 & 1,133 & $\mathrm{H} 2$ & UV & $3.98 \mathrm{E}+07$ \\
\hline 34 & 3 & 367 & 519 & 0.51 & 26 & 7.6 & 2,054 & PI & Cap & $4.54 \mathrm{E}+07$ \\
\hline 131 & 4 & 536 & 467 & 8.99 & 41 & 6.9 & 624 & PI & Cap & $4.63 \mathrm{E}+07$ \\
\hline 133 & 4 & 478 & 432 & 8.99 & 41 & 7.1 & 408 & PI & Cap & $4.35 \mathrm{E}+07$ \\
\hline 90 & 5 & 395 & 542 & 1.00 & 35 & 8.5 & 2,005 & PI & Nodulus & $5.41 \mathrm{E}+07$ \\
\hline NA & 3 & 367 & 519 & 0.51 & 26 & 7.1 & 2,267 & PI & Nodulus & NA \\
\hline 134 & 4 & 478 & 432 & 8.99 & 41 & 6.8 & 464 & PI & Nodulus & $5.12 \mathrm{E}+07$ \\
\hline 91 & 5 & 395 & 542 & 1.00 & 35 & 8.5 & 2,153 & PI & UV & $4.64 \mathrm{E}+07$ \\
\hline 132 & 4 & 536 & 467 & 8.99 & 41 & 6.4 & 271 & PI & UV & $4.66 \mathrm{E}+07$ \\
\hline 23 & 4 & 743 & 685 & 0.15 & 12 & 8.7 & 2,350 & $\mathrm{P} 0$ & Cap & $4.61 \mathrm{E}+07$ \\
\hline 28 & 4 & 804 & 580 & 1.59 & 42 & 7.5 & 2,462 & $\mathrm{P} 0$ & Cap & $4.36 \mathrm{E}+07$ \\
\hline 85 & 5 & 524 & 654 & 1.22 & 23 & 7.4 & 434 & $\mathrm{P} 0$ & Cap & $4.88 \mathrm{E}+07$ \\
\hline NA & 5 & 610 & 587 & 0.16 & 18 & 7.2 & 159 & $\mathrm{P} 0$ & Cap & $\mathrm{NA}$ \\
\hline 30 & 4 & 804 & 580 & 1.59 & 42 & 8.0 & 910 & $\mathrm{P} 0$ & Nodulus & $5.12 \mathrm{E}+07$ \\
\hline 86 & 5 & 524 & 654 & 1.22 & 23 & 7.5 & 586 & P0 & Nodulus & $5.22 \mathrm{E}+07$ \\
\hline 100 & 4 & 743 & 685 & 0.15 & 12 & 7.5 & 1,911 & P0 & Nodulus & $4.70 \mathrm{E}+07$ \\
\hline 29 & 4 & 804 & 580 & 1.59 & 42 & 7.3 & 1,562 & $\mathrm{P} 0$ & E. nodulus & $4.29 \mathrm{E}+07$ \\
\hline 61 & 4 & 467 & 536 & 0.14 & 7 & 6.1 & 357 & P0 & E. nodulus & $4.84 \mathrm{E}+07$ \\
\hline 87 & 5 & 524 & 654 & 1.22 & 23 & 7.4 & 275 & $\mathrm{P} 0$ & E. nodulus & $5.16 \mathrm{E}+07$ \\
\hline 101 & 4 & 743 & 685 & 0.15 & 12 & 8.3 & 10,630 & $\mathrm{P} 0$ & E. nodulus & $4.57 \mathrm{E}+07$ \\
\hline 2 & 5 & 780 & 666 & 0.76 & NA & 7.2 & 215 & $\mathrm{P} 0$ & UV & $4.90 \mathrm{E}+07$ \\
\hline 8 & 5 & 524 & 654 & 1.22 & 23 & 7.0 & 442 & $\mathrm{P} 0$ & UV & $5.75 \mathrm{E}+07$ \\
\hline 27 & 4 & 804 & 580 & 1.59 & 42 & 7.3 & 1,281 & $\mathrm{P} 0$ & UV & $4.31 \mathrm{E}+07$ \\
\hline 102 & 4 & 743 & 685 & 0.15 & 12 & 7.8 & 7,893 & $\mathrm{P} 0$ & UV & $5.30 \mathrm{E}+07$ \\
\hline $221 *$ & 4 & 644 & 603 & 1.02 & NA & 7.7 & 953 & P1 & Cap & $6.50 \mathrm{E}+07$ \\
\hline 111 & 5 & NA & NA & 0.17 & 49 & 7.3 & 2,810 & $\mathrm{P} 1$ & Cap & $5.27 \mathrm{E}+07$ \\
\hline 212 & 9 & 666 & 678 & 0.77 & 11 & 8.0 & 1,215 & $\mathrm{P} 1$ & Nodulus & $7.68 \mathrm{E}+07$ \\
\hline 97 & 4 & 644 & 603 & 1.02 & NA & 8.1 & 1,255 & $\mathrm{P} 1$ & Nodulus & $5.03 \mathrm{E}+07$ \\
\hline 114 & 5 & NA & NA & 0.17 & 49 & 6.9 & 3,144 & $\mathrm{P} 1$ & Nodulus & $4.54 \mathrm{E}+07$ \\
\hline 98 & 4 & 644 & 603 & 1.02 & NA & 7.4 & 2,336 & P1 & E. nodulus & $4.86 \mathrm{E}+07$ \\
\hline 113 & 5 & NA & NA & 0.17 & 49 & 6.1 & 1,895 & P1 & E. nodulus & $5.19 \mathrm{E}+07$ \\
\hline 96 & 4 & 644 & 603 & 1.02 & NA & 7.9 & 192 & P1 & Stipe & $5.13 \mathrm{E}+07$ \\
\hline 115 & 5 & 917 & 799 & 0.21 & 10 & 7.7 & 210 & $\mathrm{P} 1$ & Stipe & $6.27 \mathrm{E}+07$ \\
\hline $112 *$ & 5 & NA & NA & 0.17 & 49 & 7.9 & 1,567 & $\mathrm{P} 1$ & Upper cap & $4.81 \mathrm{E}+07$ \\
\hline $44 *$ & 5 & NA & NA & 0.17 & 49 & 7.4 & 323 & $\mathrm{P} 1$ & PV & $4.19 \mathrm{E}+07$ \\
\hline 1 & 9 & 666 & 678 & 0.77 & 11 & 7.4 & 1,524 & $\mathrm{P} 1$ & UV & $6.44 \mathrm{E}+07$ \\
\hline 99 & 4 & 644 & 603 & 1.02 & NA & 7.7 & 1,415 & $\mathrm{P} 1$ & UV & $7.50 \mathrm{E}+07$ \\
\hline
\end{tabular}


Table 9. Continued

\begin{tabular}{|c|c|c|c|c|c|c|c|c|c|c|}
\hline 64 & 5 & 1,866 & 1186 & 0.32 & 55 & 6.8 & 4,717 & $\mathrm{P} 2$ & Cap & $4.05 \mathrm{E}+07$ \\
\hline 107 & 5 & 2,001 & 1472 & 4.81 & 68 & 6.4 & 9,106 & $\mathrm{P} 2$ & Cap & $4.35 \mathrm{E}+07$ \\
\hline 118 & 5 & 1,834 & 1324 & 0.53 & 74 & 7.0 & 5,808 & $\mathrm{P} 2$ & Cap & $8.16 \mathrm{E}+07$ \\
\hline 126 & 5 & 2,091 & 1421 & 0.10 & 34 & 7.0 & 5,047 & $\mathrm{P} 2$ & Cap & $4.00 \mathrm{E}+07$ \\
\hline 55 & 5 & 1,834 & 1324 & 0.53 & 74 & 6.9 & 2,935 & $\mathrm{P} 2$ & Nodulus & $6.05 \mathrm{E}+07$ \\
\hline 69 & 5 & 1,866 & 1186 & 0.32 & 55 & 7.0 & 2,692 & $\mathrm{P} 2$ & Nodulus & $4.14 \mathrm{E}+07$ \\
\hline 77 & 5 & 2,091 & 1421 & 0.10 & 34 & 6.8 & 5,000 & $\mathrm{P} 2$ & Nodulus & $4.69 \mathrm{E}+07$ \\
\hline 108 & 5 & 2,001 & 1472 & 4.81 & 68 & 7.2 & 10,138 & $\mathrm{P} 2$ & Nodulus & $4.40 \mathrm{E}+07$ \\
\hline 62 & 5 & 1,866 & 1186 & 0.32 & 55 & 6.8 & 2,071 & $\mathrm{P} 2$ & Lamellae & $5.32 \mathrm{E}+07$ \\
\hline 106 & 5 & 2,001 & 1472 & 4.81 & 68 & 6.9 & 2,390 & $\mathrm{P} 2$ & Lamellae & $3.76 \mathrm{E}+07$ \\
\hline 116 & 5 & 1,834 & 1324 & 0.53 & 74 & 6.3 & 1,867 & $\mathrm{P} 2$ & Lamellae & $5.36 \mathrm{E}+07$ \\
\hline 124 & 5 & 2,091 & 1421 & 0.10 & 34 & 6.8 & 304 & $\mathrm{P} 2$ & Lamellae & $4.71 \mathrm{E}+07$ \\
\hline 40 & 5 & 2,001 & 1472 & 4.81 & 68 & 7.4 & 3,583 & $\mathrm{P} 2$ & C. stipe & $6.08 \mathrm{E}+07$ \\
\hline 67 & 5 & 1,866 & 1186 & 0.32 & 55 & 7.0 & 1,892 & $\mathrm{P} 2$ & C. stipe & $5.58 \mathrm{E}+07$ \\
\hline 68 & 5 & 1,866 & 1186 & 0.32 & 55 & 6.7 & 7,474 & $\mathrm{P} 2$ & E. nodulus & $4.80 \mathrm{E}+07$ \\
\hline 76 & 5 & 2,091 & 1421 & 0.10 & 34 & 7.1 & 8,197 & $\mathrm{P} 2$ & E. nodulus & $4.10 \mathrm{E}+07$ \\
\hline 109 & 5 & 2,001 & 1472 & 4.81 & 68 & 6.5 & 17,474 & $\mathrm{P} 2$ & E. nodulus & $5.95 \mathrm{E}+07$ \\
\hline 121 & 5 & 1,834 & 1324 & 0.53 & 74 & 6.9 & 6,215 & $\mathrm{P} 2$ & E. nodulus & $3.78 \mathrm{E}+07$ \\
\hline 39 & 5 & 2,001 & 1472 & 4.81 & 68 & 7.1 & 7,425 & $\mathrm{P} 2$ & Stipe & $6.45 \mathrm{E}+07$ \\
\hline 66 & 5 & 1,866 & 1186 & 0.32 & 55 & 6.7 & 6,589 & $\mathrm{P} 2$ & Stipe & $5.95 \mathrm{E}+07$ \\
\hline 120 & 5 & 1,834 & 1324 & 0.53 & 74 & 7.4 & 1,167 & $\mathrm{P} 2$ & Stipe & $6.43 \mathrm{E}+07$ \\
\hline 128 & 5 & 2,091 & 1421 & 0.10 & 34 & 7.5 & 4,607 & $\mathrm{P} 2$ & Stipe & $5.42 \mathrm{E}+07$ \\
\hline 38 & 5 & 2,001 & 1472 & 4.81 & 68 & 6.6 & 6,379 & $\mathrm{P} 2$ & Upper cap & $5.97 \mathrm{E}+07$ \\
\hline 65 & 5 & 1,866 & 1186 & 0.32 & 55 & 7.2 & 3,300 & $\mathrm{P} 2$ & Upper cap & $3.30 \mathrm{E}+07$ \\
\hline 119 & 5 & 1,834 & 1324 & 0.53 & 74 & 7.1 & 5,103 & $\mathrm{P} 2$ & Upper cap & $4.16 \mathrm{E}+07$ \\
\hline 127 & 5 & 2,091 & 1421 & 0.10 & 34 & 7.1 & 3,841 & $\mathrm{P} 2$ & Upper cap & $4.25 \mathrm{E}+07$ \\
\hline 37 & 5 & 2,001 & 1472 & 4.81 & 68 & 7.7 & 1,914 & $\mathrm{P} 2$ & PV & $4.45 \mathrm{E}+07$ \\
\hline 63 & 5 & 1,866 & 1186 & 0.32 & 55 & 7.4 & 1,757 & $\mathrm{P} 2$ & PV & $4.14 \mathrm{E}+07$ \\
\hline 117 & 5 & 1,834 & 1324 & 0.53 & 74 & 6.9 & 1,588 & $\mathrm{P} 2$ & PV & $5.52 \mathrm{E}+07$ \\
\hline 125 & 5 & 2,091 & 1421 & 0.10 & 34 & 7.1 & 882 & $\mathrm{P} 2$ & PV & $3.83 \mathrm{E}+07$ \\
\hline 110 & 5 & 2,001 & 1472 & 4.81 & 68 & 7.3 & 1,474 & $\mathrm{P} 2$ & UV & $3.96 \mathrm{E}+07$ \\
\hline 122 & 5 & 1,834 & 1324 & 0.53 & 74 & 6.5 & 507 & $\mathrm{P} 2$ & UV & $5.20 \mathrm{E}+07$ \\
\hline 123 & 5 & 1,866 & 1186 & 0.32 & 55 & 6.3 & 740 & $\mathrm{P} 2$ & UV & $4.98 \mathrm{E}+07$ \\
\hline 129 & 5 & 2,091 & 1421 & 0.10 & 34 & 7.2 & 1,472 & $\mathrm{P} 2$ & UV & $4.32 \mathrm{E}+07$ \\
\hline
\end{tabular}

\subsubsection{Analyzing RNA-seq data}

\subsubsection{Read quality check and trimming}

On average, 49.96 million and 142 base pairs long reads were produced per sample $(\mathrm{n}=84)$ using Illumina sequencing (Figure 31.). Both the average read length and read number decreased after quality trimming to, on average, 130 base pairs and 43.65 million reads, respectively. On average, 37.18 million reads (74,6\%) were uniquely mapped to the reference genome of Coprinopsis cinerea by using a two-round mapping of the STAR alignment program. $82.7 \%$ of the uniquely mapped reads were mapped to exonic regions. The rest of the reads were distributed across intronic regions (8.6\%), rRNA sequences (8\%), and the ERCC external RNA control reference sequences $(0.7 \%)$ (Figure 31.). 
A)

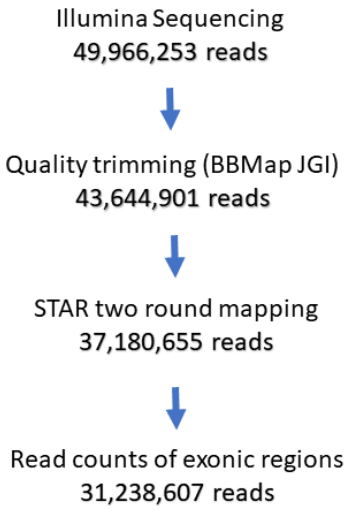

B)

\section{Uniquely mapped reads}

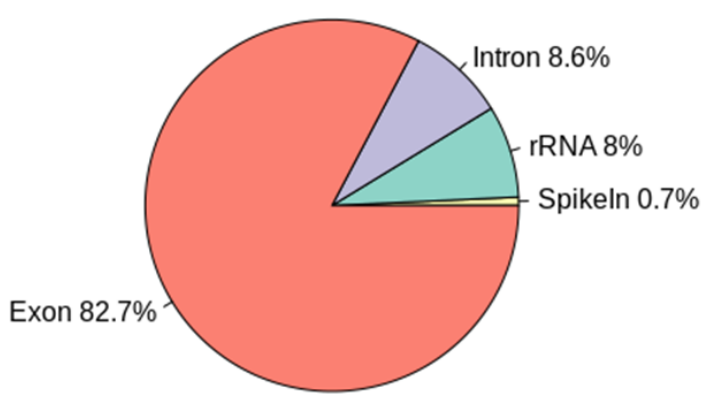

Figure 31. Statistics of the 84 samples through the quality trimming and read aligning to the reference genome. A) The average number of reads through the read processing steps. B) The distribution of the uniquely mapped reads across exonic regions, intronic regions, rRNA sequences and external RNA control sequences.

Out of the 14,337 genes, 14,242 genes were found to which at least one read mapped in at least one of the 84 samples. By filtering low expressed genes (sum of reads across all samples per gene >=10), 12,903 genes were left in our dataset. After uniquely mapped reads were transformed to count data, two normalization steps were performed. First, a full quantile library size normalization was applied, which is robust to outliers. Second, based on the GC content distribution of reads and read counts, we found that cDNA amplification could cause biases (Figure 32.). Therefore, a GC content normalization was performed, with the aim to eliminate expression level biases caused by cDNA amplification. GC normalization successfully removed differences between samples except in genes with low and high GC content (Figure 32.). However, only 83 genes possess deviant GC content, therefore, we think that the small number of these genes per sample made the normalization procedure problematic at this region and we did not exclude these genes from subsequent analyses. 

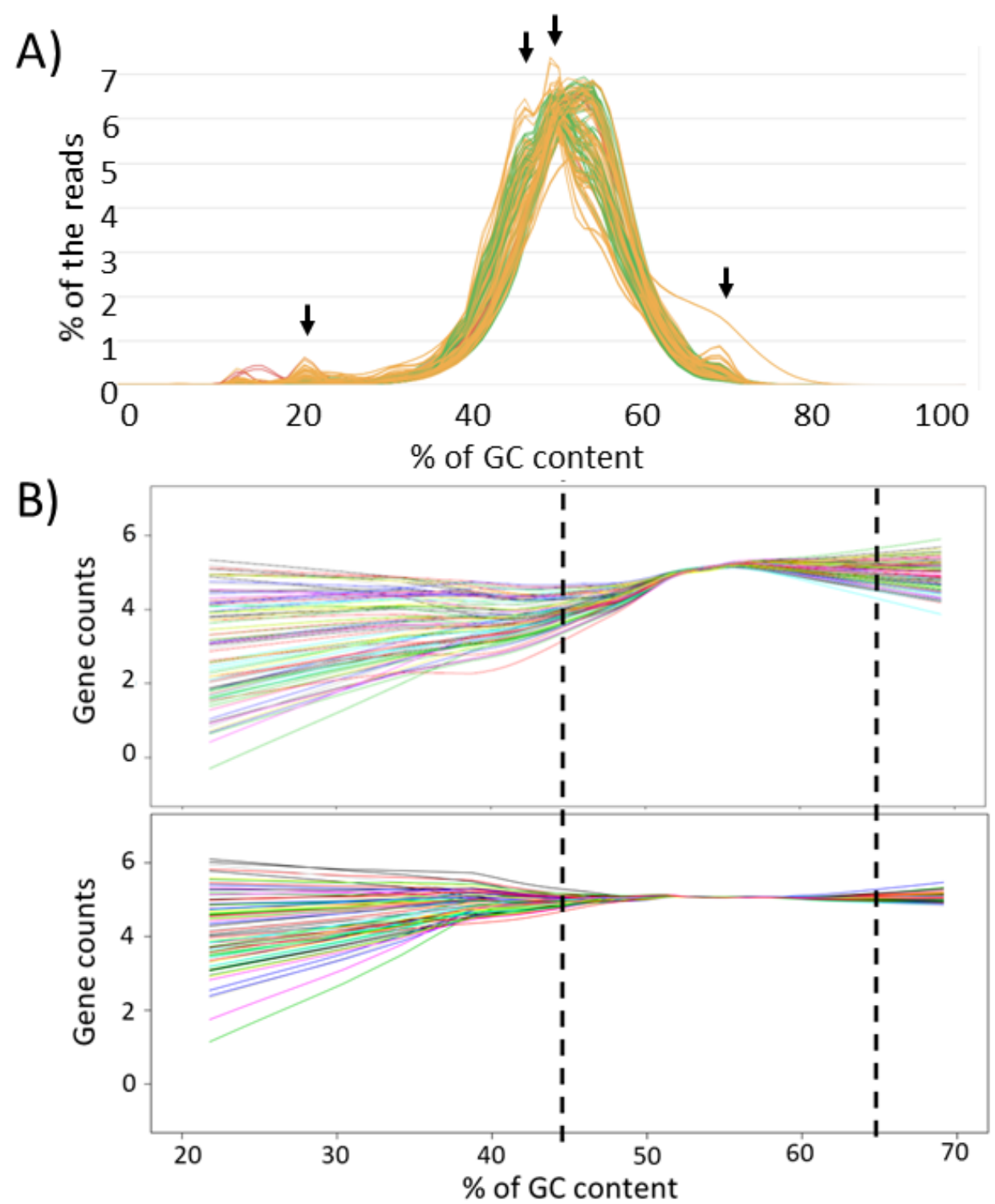

Figure 32. Quality check and normalization procedures of the RNA-seq data. (A) The distribution of reads as a function of GC content. Arrows indicate regions where reads were enriched in certain samples. (B) The mean gene count data distribution as a function of GC content. The upper panel shows the gene counts after library size but before GC content normalization. The lower panel shows the gene counts after both library size and GC content normalization. Dashed lines represent low and high GC content regions to which only 83 genes belong.

\subsubsection{Detecting outlier samples}

We examined four main statistics (PCA, number of drop out events, spike-in statistics, Cook's distance) to characterize and detect outlier samples. Next, the results of the main statistics are described, following our conclusions on samples essential to be excluded. After the normalization procedures, we performed PCA on all samples $(n=84)$ using $\log 2$ normalized counts of genes. The first two principal components explained $37.17 \%$ of the variance of the dataset. In general, the samples grouped by tissue types rather than by developmental stages (Figure 33.). However, we found six potential outlier 
samples (ID 44, 93, 95, 103, 104, 112) because they were positioned distant from other replicates of their sample type.
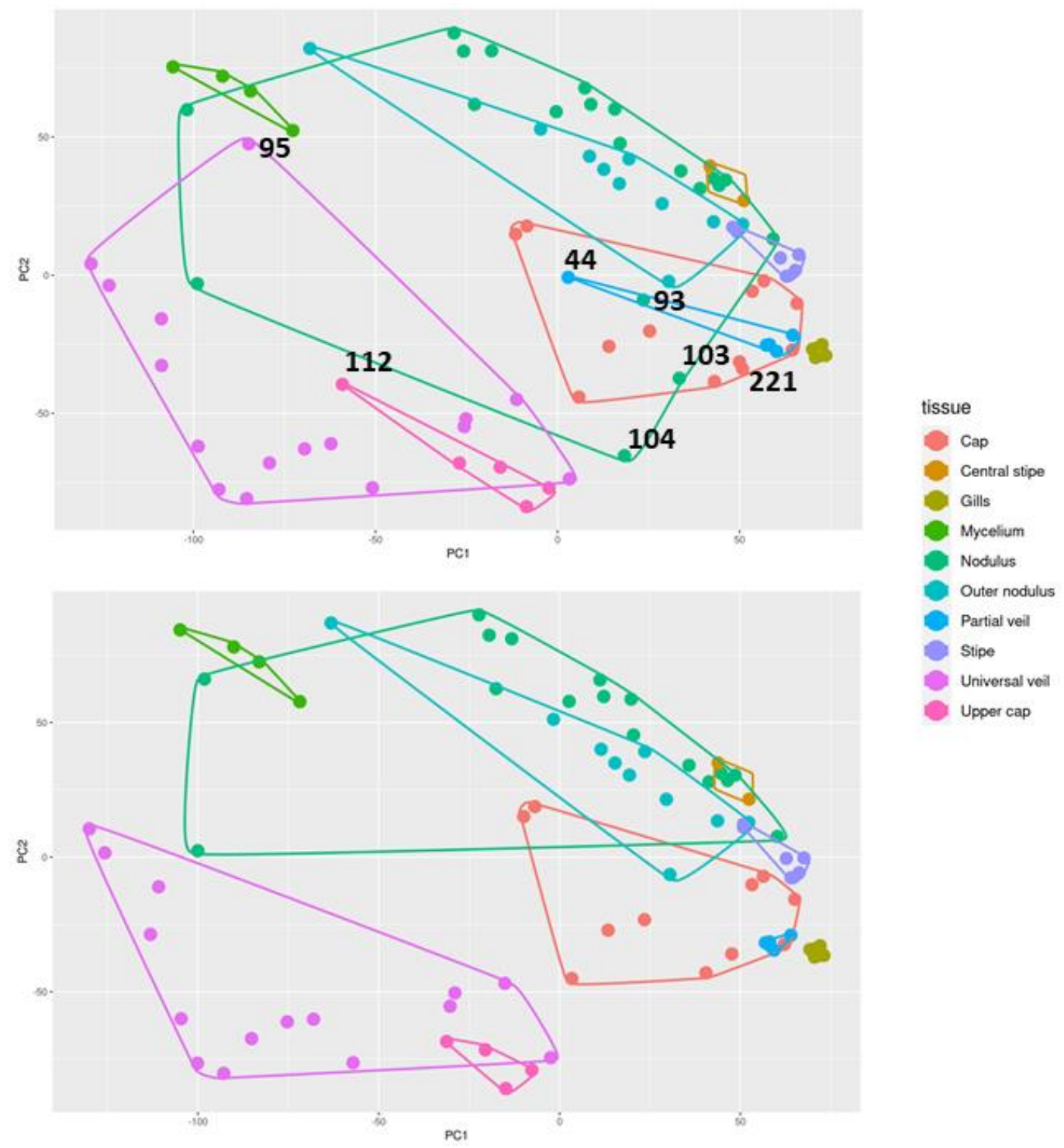

Figure 33. Principal component analyses of the RNA-seq data. The top panel shows the PCA of the 84 samples, while the bottom panel shows the PCA of 77 samples. Data points are colored and encircled based on the tissue types. Numbers indicate the ID number of the samples, which were excluded from further analyses.

To further characterize samples, we evaluated the number of genes in each sample, to which no read mapped at all (i.e., drop out events). We found that one sample (ID: 221) possessed considerably more drop out events $(5,182$ genes) than other samples did (mean $=1,237$ genes). Next, we examined three statistics of the external ERCC Spike-in RNA control (Baker et al., 2005; Reid, 2005). We found that in three samples (IDs: 86, 124, 221) less than $40 \%$ of the RNA spike-in sequences were recovered. In two samples (IDs: $92,221)$ the Pearson correlation coefficient between the log number of reads vs. $\log$ 
starting concentration was lower $\left(r^{2}<0.67\right)$ than in most of the samples (mean $\left.r^{2}=0.9\right)$. We also found that the lower limit of detection was considerably higher for one sample (LDD: 2,190,062 control molecules per $100 \mathrm{ng}$ poly(A) RNA, ID: 212). Finally, four samples (IDs: 44, 111, 112, 212) possessed more distorting genes than other samples based on the distribution of Cook's distance in a likelihood-based GLM analysis. By summarizing all the statistics mentioned above, we decided to exclude seven samples (Table 10.), and we performed all the subsequent analyses on this subset containing 77 samples.

Table 10. The seven samples were excluded from the dataset. "Yes" means that we experienced bias or anomaly based on the given statistics.

\begin{tabular}{ccccc} 
Sample ID & PCA & $\begin{array}{c}\text { Drop out } \\
\text { event }\end{array}$ & $\begin{array}{c}\text { ERCC } \\
\text { spike-in }\end{array}$ & $\begin{array}{c}\text { Cook's } \\
\text { distance }\end{array}$ \\
\hline 221 & - & Yes & Yes & - \\
112 & Yes & - & - & Yes \\
44 & Yes & - & - & Yes \\
103 & Yes & - & - & - \\
104 & Yes & - & - & - \\
93 & Yes & - & - & - \\
95 & Yes & - & - & -
\end{tabular}

The most exhaustive data was available for stage 2 primordium. In contrast, in stage 1 primordium, we could not manage to retrieve RNA-seq data for the upper cap tissue and a low number of replicates (1-2) were available for other tissues (Table 11.). One vegetative mycelium was processed differently than the other samples. It was not gathered by a needle but was laser micro-dissected next to primary hyphal knot tissues. Despite these differences, the four replicates of vegetative mycelium showed high similarities on PCA plots (Figure 33.), meaning that the different sampling methods did not affect the outcome of the RNA-seq. 
Table 11. The number of replicates used in subsequent analyses. Empty cells represent combinations where the given tissue does not exist. * Note that one vegetative mycelium sample was retrieved from sections containing primary hyphal knots. VM=vegetative mycelium, $\mathrm{HI}=$ primary hyphal knot, $\mathrm{H} 2=$ secondary hyphal knot, $\mathrm{PI}=$ primordium initial, $\mathrm{PO}=$ Stage $\mathrm{O}$ primordium, $P 1=$ Stage 1 primordium, $P 2=$ Stage 2 primordium

\begin{tabular}{llllllll} 
& VM & H1 & H2 & PI & P0 & P1 & P2 \\
\hline VM & 3 & $1^{*}$ & - & - & - & - & - \\
Nodulus & & 2 & 3 & 3 & 3 & 3 & 4 \\
Universal veil & & & 3 & 2 & 4 & 2 & 4 \\
Cap & & & & 3 & 4 & 1 & 4 \\
Outer nodulus & & & & 4 & 2 & 4 \\
Upper cap & & & & & 0 & 4 \\
Stipe & & & & & & 2 & 4 \\
Partial veil & & & & & & 4 \\
Central stipe & & & & & & 2 \\
Lamellae & & & & & & & \\
& & & & & & &
\end{tabular}

\subsubsection{Differentially expressed genes at the transition from vegetative mycelium to the fruiting body}

Fruiting body development of $C$. cinerea starts with the formation of primary hyphal knot (H1), which can be considered as a complex multicellular structure (threedimensional organization, not all cells are in direct contact with the environment). From the H1 stage, two developmental decisions are made towards sclerotium or fruiting body. Therefore, the secondary hyphal knot $(\mathrm{H} 2)$ can be considered as the first developmental stage, which is committed towards fruiting body development. The examination of the first two developmental stages is essential to reveal the genetic background of the transition from simple to complex multicellularity and the initial steps of fruiting body development. We performed differential expression analysis on three tissue types, H1, $\mathrm{H} 2$ nodulus, and $\mathrm{H} 2$ universal veil using the vegetative mycelium (VM) as a reference sample. We found that a high number of genes were upregulated in H1 (1,293 genes), H2 universal veil $(2,050)$, and $\mathrm{H} 2$ nodulus $(2,590)$ relative to VM.

We performed enrichment analyses on IPR, KOG and GO terms within each upregulated geneset of the three tissue types. The analyses provided significantly enriched terms (adj. $\mathrm{p}<0.05)$ with only general metabolic and broad functions in $\mathrm{H} 1$ and $\mathrm{H} 2$ universal veil tissues (Electronic Appendix 5.). However, in $\mathrm{H} 2$ nodulus, more specific terms were significantly enriched related to signaling, cell wall remodeling, DNA 
replication such as 'kinases', 'glucan 1,4-alpha-glucosidase activity', 'ATP-dependent 5'3' DNA helicase activity' (for the full list see Electronic Appendix 6.)

Based on previous studies, we know that the hyphal growth pattern of the mycelium is changing through fruiting body formation. Genes showing upregulation and playing role in the control of hyphal growth pattern and cell morphology could be potential participants of the evolution of complex multicellularity. That is why we asked if changes in hyphal growth patterns and cell morphology could be seen at the transcriptome level. To do that, we manually curated the annotations of upregulated genes related to cell-cycle control, mitotic activity, hyphal branching, septum formation, anastomosis, cell adhesion, cell wall biogenesis and remodeling (see Materials and Methods and Electronic Appendix 7.). Overall, 161 genes were upregulated in the first two developmental stages which could be associated with cell division (cell cycle control and mitosis) and hyphal growth patterns (branching, septation, anastomosis) (Appendix 2). We found an upregulation of genes associated with cell cycle control (37 genes, e.g., Cyclins, Anaphase-promoting complex, CDK proteins), with mitotic processes (69 genes, e.g., spindle body organization, DNA replication and repair), with hyphal fusion (3 genes, two STRIPAK proteins, Rac1) and with septation (9 genes, e.g., septins). Further, the number of cell division and hyphal growth-related genes were significantly increased from $\mathrm{H} 1$ to $\mathrm{H} 2$ (Fisher exact test: $\mathrm{p}<0.05$, Figure 34.).

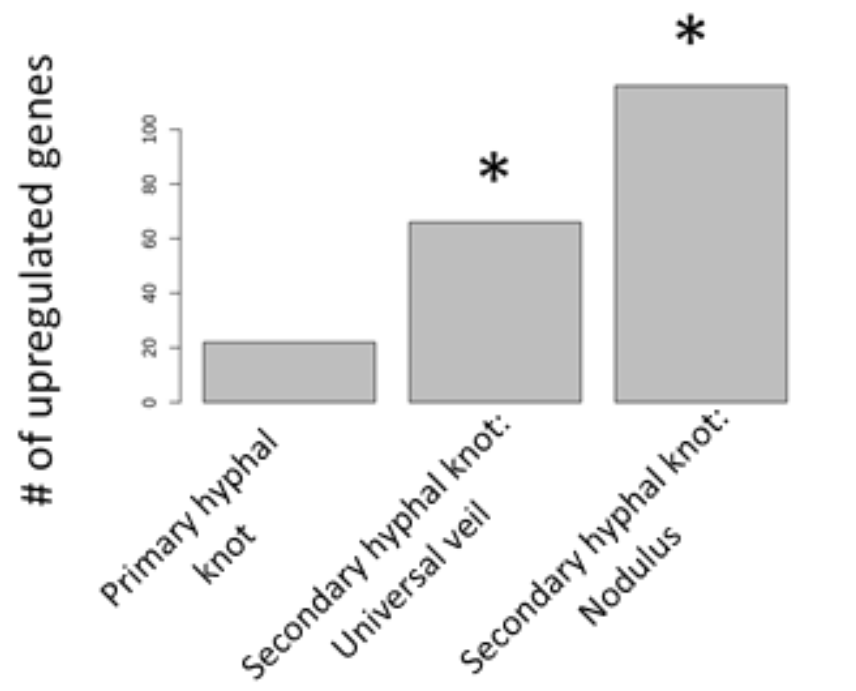

Figure 34. Increased number of genes were detected among the cell division and hyphal growth pattern related genes in the secondary hyphal knot relative to the primary hyphal knot. Asterisk: Fisher exact test, $p<0.05$ 
Some of these cell division and hyphal growth pattern associated genes were examined by previous studies (discussed in detail in the paragraph 5.). For example, phosphatidylinositol-3-kinase-related kinase (Atr1) and a serine-threonine kinase (Chk1) were examined in a gene-silencing experiment of $C$. cinerea (de Sena-Tomás et al., 2013). In our study, we found that the Atr1 (Copci_AmutBmut1_19106) was significantly upregulated in the nodulus tissue of the secondary hyphal knot (Figure 35., shrunken LFC: 1.1 , weighted adj. $\mathrm{p}<0.05)$. However, this gene was not expressed differentially in the primary hyphal knot and the differential expression of the Chk1 gene (Copci_AmutBmut1_450167) was not detected either in the primary or the secondary hyphal knot. A common pattern in the expression profiles of the Atr1 and Chk1 genes was that the expression in universal veil tissues was generally low. This pattern could be detected in other cell division related genes as well (Figure 35.). For example, a gene (Copci_AmutBmut1_360237), which has an ortholog in S. cerevisiae (Prp19 gene) showed high expression in almost all tissues, but starting from the primordium initial stage, it showed low expression level in the universal veil. Prp19 is the part of the NTC/Prp19c protein complex whose primary function is in splicing. However, it was showed that the Prp19 has both an indirect and direct function in genome maintenance and cell-cycle progression (Chanarat and Sträßer, 2013). A similar pattern could be noticed in two other genes as well. The Copci_AmutBmut1_368398 gene with a HORMA domain has an ortholog in the $S$. cerevisiae (MAD2), which is the part of the spindle checkpoint machinery. The Copci_AmutBmut1_474965 gene has an OB-fold motif that could bind nucleic acids. GO and KOG terms ('DNA recombination'; 'regulation of S phase of mitotic cell cycle'; 'DNA recombinational repair protein BRCA2') also indicate that this gene could have a function in cell division. 


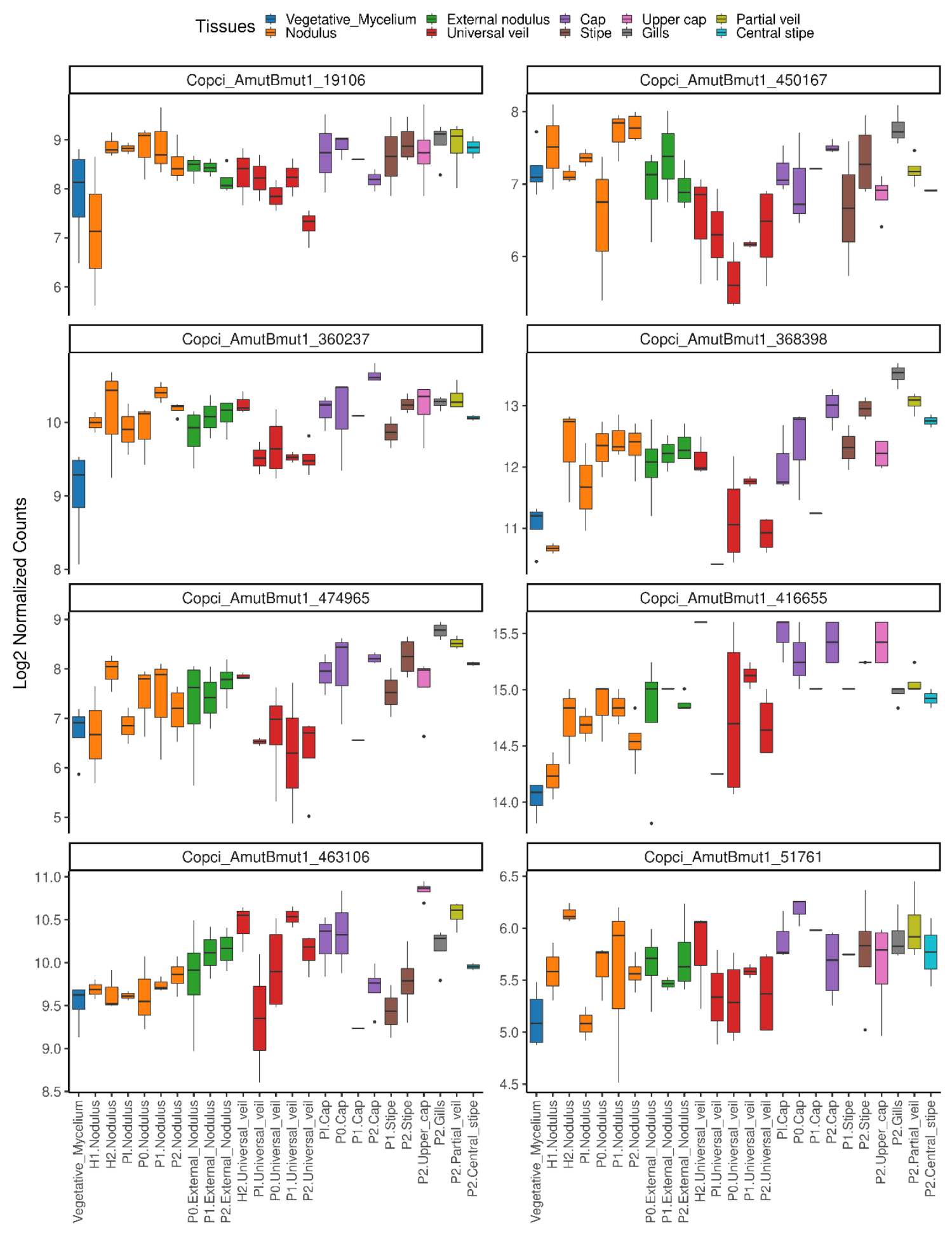

Figure 35. Gene expression profiles of various genes. First row. Atr1 (Copci_AmutBmut1_19106) and Chk1 (Copci_AmutBmut1_450167) genes. Second and third rows. Prp19 gene (Copci_AmutBmut1_360237), a gene with HORMA domain (Copci_AmutBmut1_368398) and a gene (Copci_AmutBmut1_474965) with putative function in mitotic cell cycle. Third and fourth rows. A rac-1 ortholog (Copci_AmutBmut1_416655) and two members of the STRIPAK complex: PP2A, (Copci_AmutBmut1_463106) and SLAMP (Copci_AmutBmut1_51761) 
We found three genes that were upregulated in the secondary hyphal knot and are putatively associated with hyphal anastomosis (Figure 35.). One gene (Copci_AmutBmut1_416655) is the ortholog of rac-1 in Neurospora crassa, which is part of the MAP kinase pathway. The other two genes are part of the striatin-interacting phosphatases and kinases (STRIPAK) complex (Bloemendal et al., 2012). We observed that, one of the proteins of the catalytic subunit of the STRIPAK complex (PP2A, Copci_AmutBmut1_463106) was upregulated in the universal veil, while the membrane anchoring subunit (SLAMP, Copci_AmutBmut1_51761) was upregulated in the nodulus. We did not find an explicit expression pattern indicating that three hyphal anastomosis associated genes would have higher expression in more compact tissues such as nodulus or cap than in loosely structured tissues like universal veil or stipe tissues. However, none of the three genes were upregulated in the hyphal knot stage, suggesting that hyphal anastomosis is not as frequent in the first stage than in later stages. Moreover, the rac-1 ortholog gene showed high expression in the cap, while the PP2A ortholog gene showed low expression in the stipe indicating the role of these genes in modulating the anatomy of tissues.

Based on our manually curated functional categories (Electronic Appendix 6.), we also found 104 genes related to cell wall biogenesis and remodeling, among them there were 75 genes with putative glycoside hydrolase (GH) activity. The highest number of upregulated genes was observed in the GH61/AA9 family $(n=13)$ followed by the GH5 $(n=10)$, GH16 $(n=9)$ and GH18 $(n=6)$ families. Many of them were canonical cell wall remodeling genes such as chitinases (GH18) or endo-1,3- $\beta$-glucanases (GH16). However, 13 copper-containing lytic polysaccharide mono-oxygenases (LPMOs, AA9 family) were also significantly upregulated in the primary and secondary hyphal knots showing different expression profiles across tissues and developmental stages (Figure 36.). 


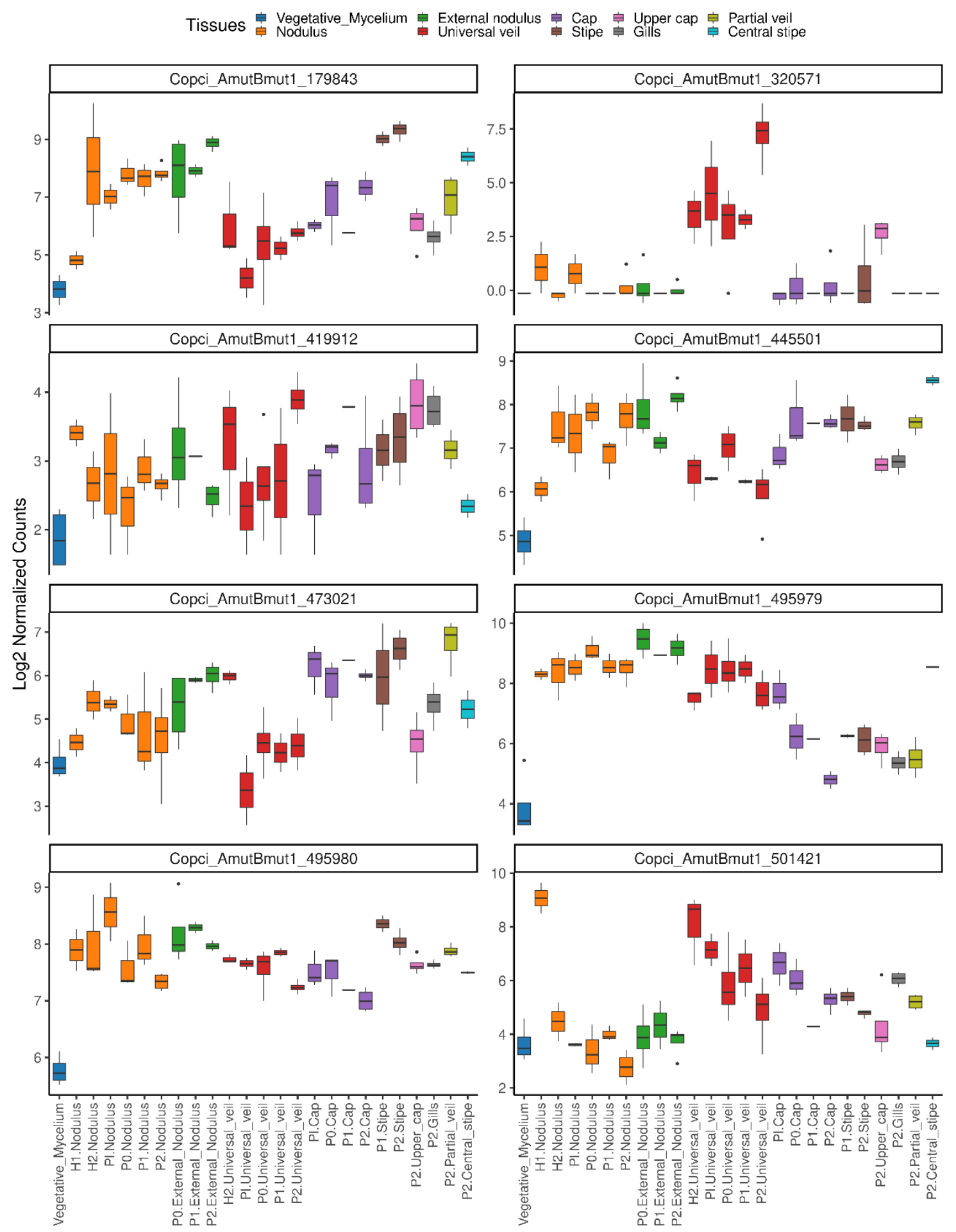

Figure 36. The gene expression profile of nine LPMO coding genes. Copci_AmutBmut1_320571 gene showed a clear specificity for universal veil. Copci_AmutBmut1_501421 gene exhibited high expression in the universal veil and cap related tissues, while low expression in nodulus related tissues. In case of Copci_AmutBmut1_179843, Copci_AmutBmut1_445501 and Copci_AmutBmut1_473021 genes, high expression level was detected in the nodulus related tissues but relatively low in the universal veil. 
It is known that thickness of the cell wall and the beta-glucan content of the hyphal knots were increased relative to the mycelium (Kamada and Tsuru, 1993; Xie et al., 2020). Therefore, we searched for terms such as beta-glucan synthase, beta-glucanase and beta-glucosidase in our database to summarize genes with beta-glucan metabolism. We found 16 genes belonging to the GH1 (1 gene), GH3 (4 genes), GH16 (5 genes) and GH5 (6 genes) families (Appendix 3). Interestingly, the same number of beta-glucan anabolic and catabolic genes were upregulated in the primary hyphal knot and the nodulus of the secondary hyphal knot. This finding suggests that the cell wall thickness is continuously modified in the hyphal knots.

\subsubsection{Tissue enhanced genes}

We performed differential expression analyses using a deviation GLM contrast which revealed genes that had higher gene expression in a given tissue than the mean expression of all tissues in a given stage. In this study we focused on tissues which were examined in the macro-evolutionary analyses and showed that the presence of the cap, the universal veil, the partial veil, and the increased surface area of the hymenophore were elevated the diversification of mushroom species. We found that 2,053, 1,053, 1,260 and 941 genes were enhanced in the universal veil, lamellae, cap and the partial veil in at least one stage, respectively (Electronic Appendix 8-10). Among the tissue enhanced genes, 1,294 were shared by two or more tissues meaning that $26 \%$ of all tissue enhanced genes play a role in the development of more tissues (Figure 37.). 


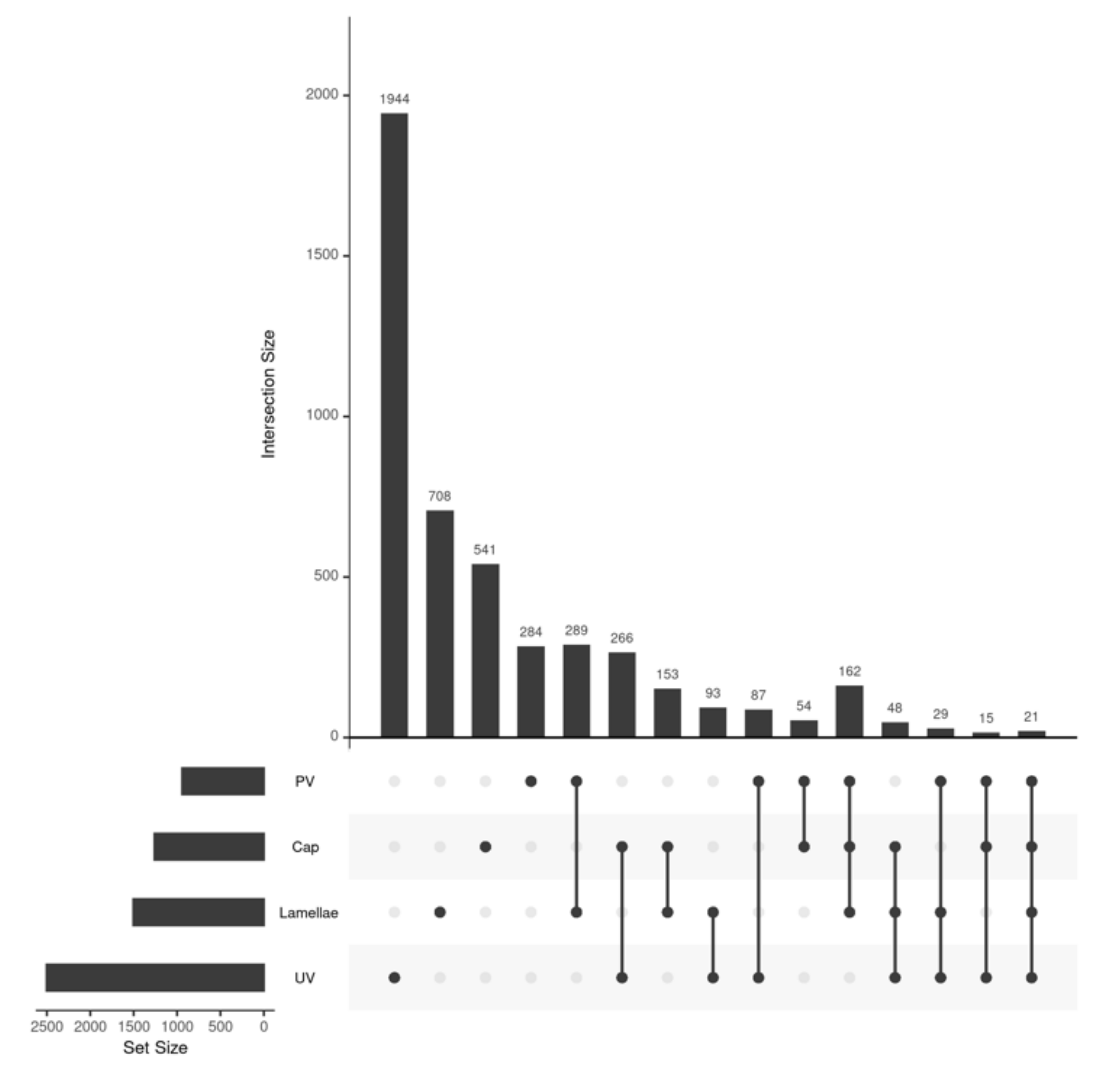

Figure 37. Intersections of tissue enhanced genes. Rows are depicting the number of tissue enhanced genes. Columns show the number of tissue enhanced genes in a given intersection of the tissues. $P V=$ partial veil, $U V=$ universal veil.

We also examined the distribution of genes across stages, but among the four tissues examined in this study two occur in more than one stages. We found that twice as many tissue enhanced genes were shared by different stages in the universal veil (28\%) than in the cap (15\%). This implies that the cap tissue is more actively changing through the development than the universal veil, with the latter potentially staying constant to some extent (Figure 38.). 


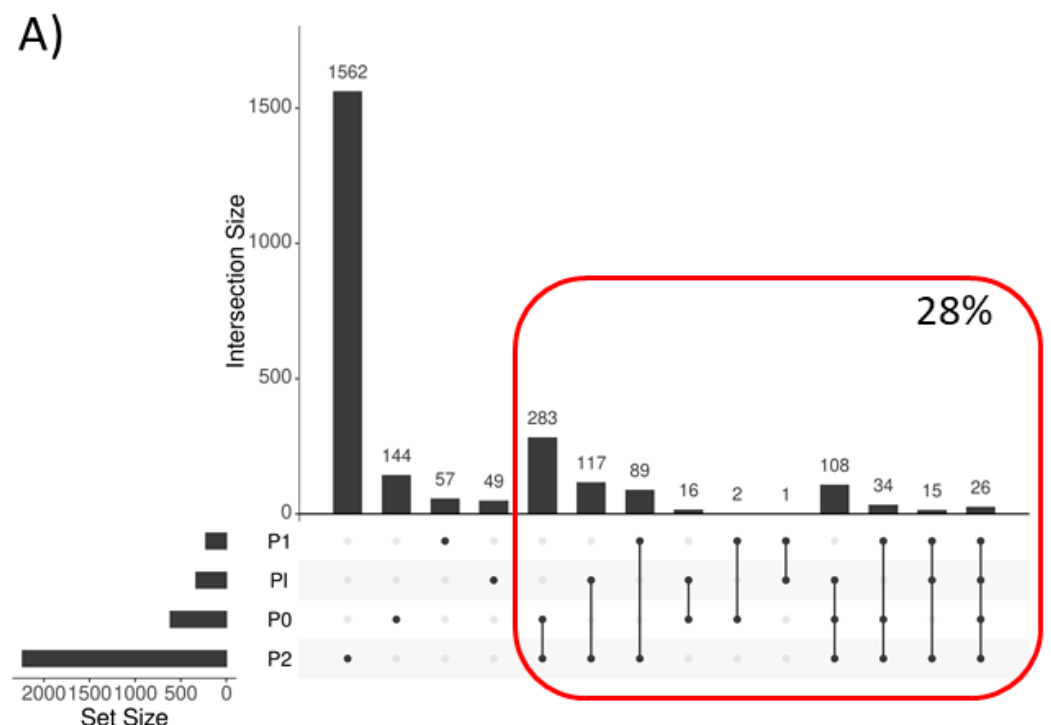

Set Size

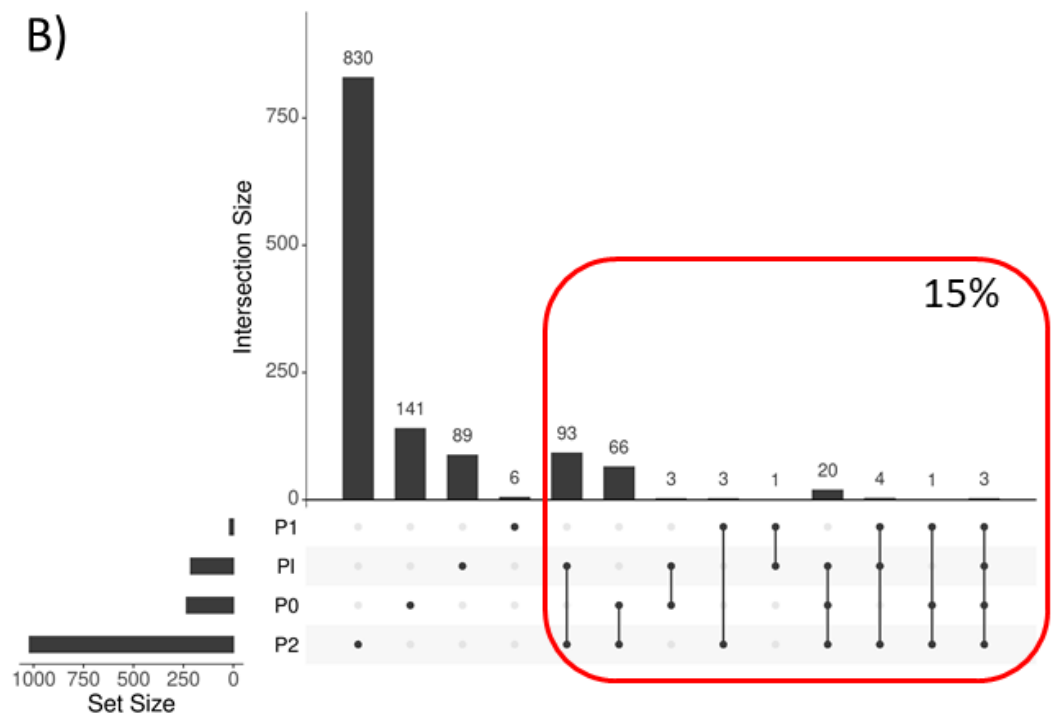

Figure 38. Intersections of tissue enhanced genes across different developmental stages. Rows represent the number of tissue enhanced genes in a given developmental stage. Columns depict the number of genes in a given intersection. (A) Universal veil enhanced genes. (B) Cap enhanced genes. Red rectangles show the gene sets which are shared by at least two developmental stages. The percentages represent the ratio of shared genes to all enhanced genes within a tissue. PI = Primordium initial, $P 0=$ Stage 0 primordium, $P 1=$ Stage 1 primordium, $P 2=$ Stage 2 primordium.

Some specific GO, IPR and KOG terms were enriched among cap and universal veil enhanced genes such as surface coating and defense-related categories ('hydrophobin', 'ricin b like lectin', 'cerato-platanin', 'adhesion', 'peptidase inhibitor I66') (Electronic Appendix 11-12). The partial veil exhibited enriched terms such as 'replication', 'structural constituent of the cell wall' and 'hydrophobin'. Lamellae could be characterized, among others, by the enrichment of transcription factors (defined as 
Krizsán et al., 2019), replication related terms, 'glycoside hydrolase family 7', and 'fungal-type protein kinase' (Electronic Appendix 10).

We hypothesized that one of the main functions of the universal and partial veil as well as the cap tissues is the protection and defense of the developing spores. Therefore, we checked whether a high number of genes associated with defense or providing a hydrophobic surface to hyphae could be found in these tissues. Based on limited literature data (Hamann et al., 2008; Hardwick, 2018), we further hypothesized that genes related to programmed cell death are more active at the formation of lamellae than in other tissue types. To do that, we gathered genes using searching terms across all functional annotations (Table 4.) then manually curated lists. We included functional categories in this analyses related with cell division and hyphal morphogenesis as well to have reference points, thus we assigned one of the following terms to genes: defense, surface coating, programmed cell death, cell-cycle control, mitotic functions, hyphal growth pattern (septation, branching, hyphal fusion), cell wall biogenesis, cell wall remodeling (Electronic Appendix 7). To compare the representation of a functional group across tissue enhanced genes, we calculated the proportion of genes with a functional term in a tissue.

The mitotic function was represented with the highest proportion within the cap, the lamellae and the partial veil enhanced genes. The second biggest functional group was cell wall remodeling $(2.2-3.3 \%$ of the given tissue enhanced genes), which was the top term in the universal veil (Figure 39.). 


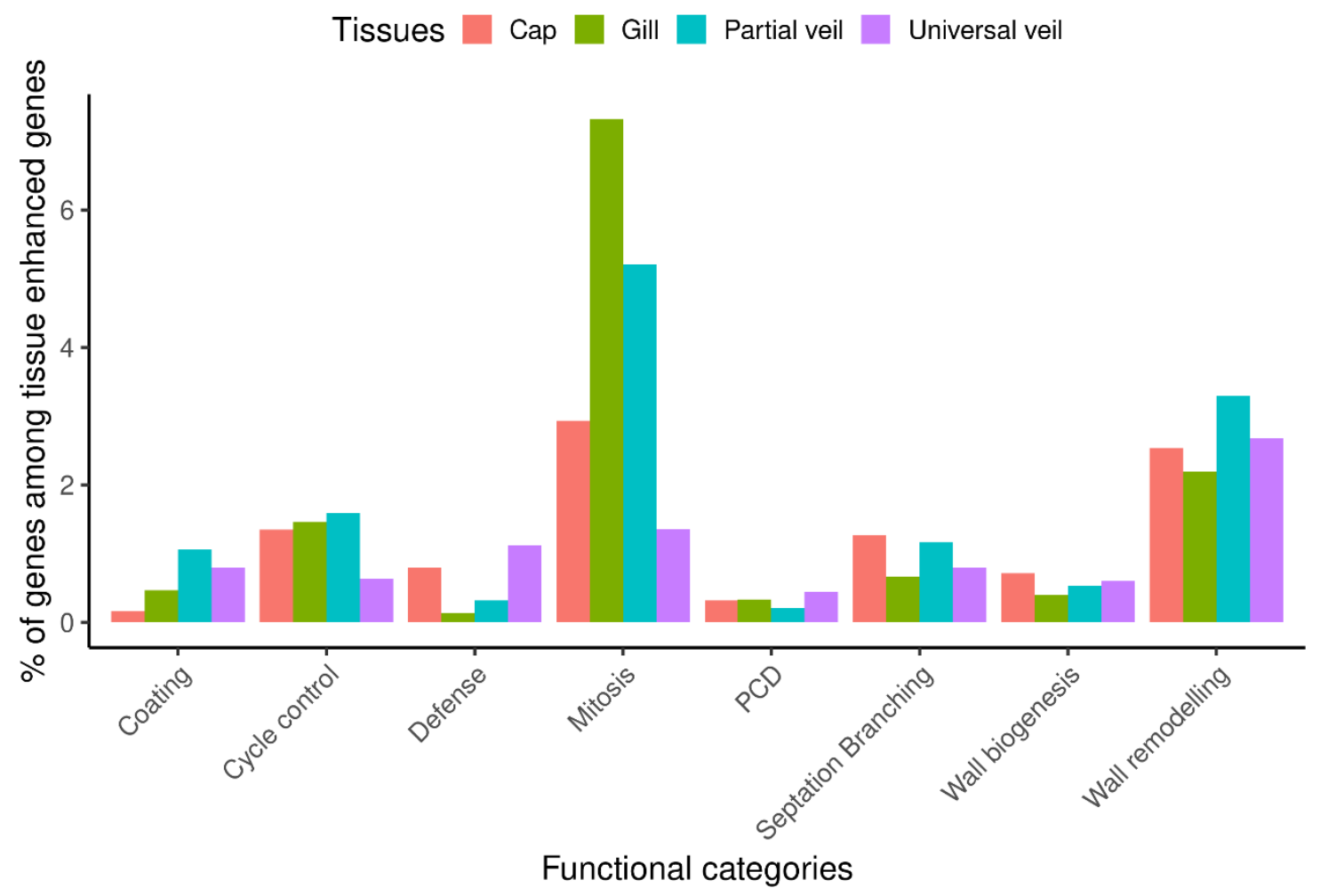

Figure 39. The percentage of genes with a given functional category within and across tissue enhanced genes. $P C D=$ programmed cell death

To further disentangle the two most frequent functional categories among tissues we also calculated the intersections of mitosis or cell wall remodeling genes across different tissue types (Figure 40.). We found that mitosis related genes were shared across different tissues to a larger extent than cell wall remodeling genes. This suggests that mitosis related tissue enhanced genes are more general across different tissue types of primordia than cell wall remodeling related tissue enhanced genes. 


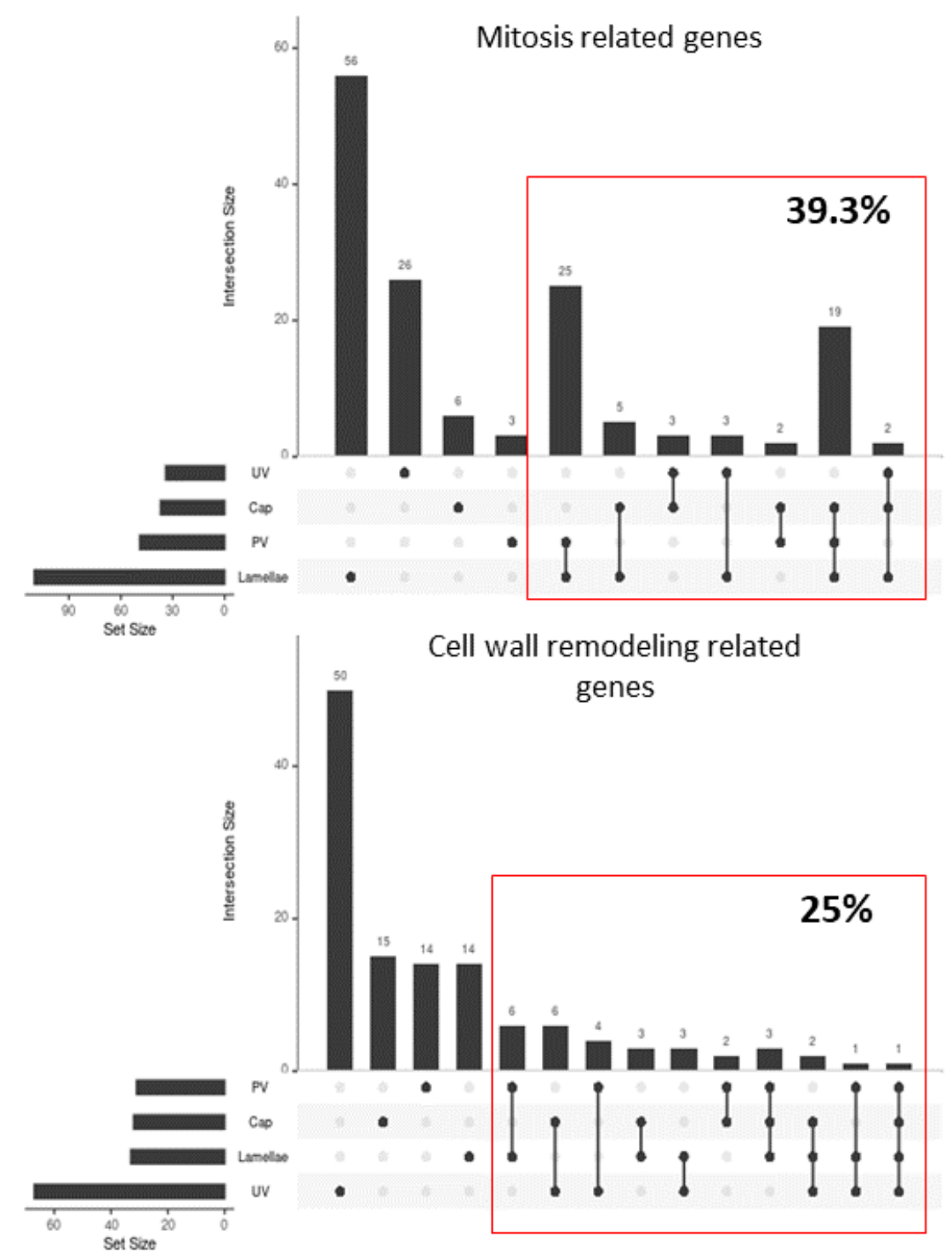

Figure 40. Intersections of tissue enhanced genes within mitosis related genes (upper panel) and cell wall remodeling related genes (lower panel). Rows represent the number of tissue enhanced genes. Columns depict the number of genes in a given intersection. Red rectangles show the gene sets which are shared by at least two tissue types. The percentages represent the ratio of shared genes to all enhanced genes within a functional category. $P V=$ partial veil, $U V=$ universal veil.

By focusing on our hypothesis that the universal and partial veil as well as the cap tissues give protection against environmental impacts we found that defense-related genes were represented with a higher percentage in the cap and universal veil than in other tissues (Figure 41.). Most typical defense-related terms were the 'copsin', 'aerolysin-like toxin', 'ricin B-like lectins', 'peptidase inhibitor I66' and 'isopenicillin N synthase' in the cap and universal veil tissue enhanced genes. Surface coating genes were more characteristic for partial veil and universal veil than for other tissues. Numerous 'hydrophobins', 'wax synthases' and 'cerato-platanin' encoding genes were identified in these tissues. Interestingly we did not find pronounced differences in the proportion of 
programmed cell death (PCD) related genes across the four tissue types, suggesting that PCD was not more active in lamellae than in other tissues.

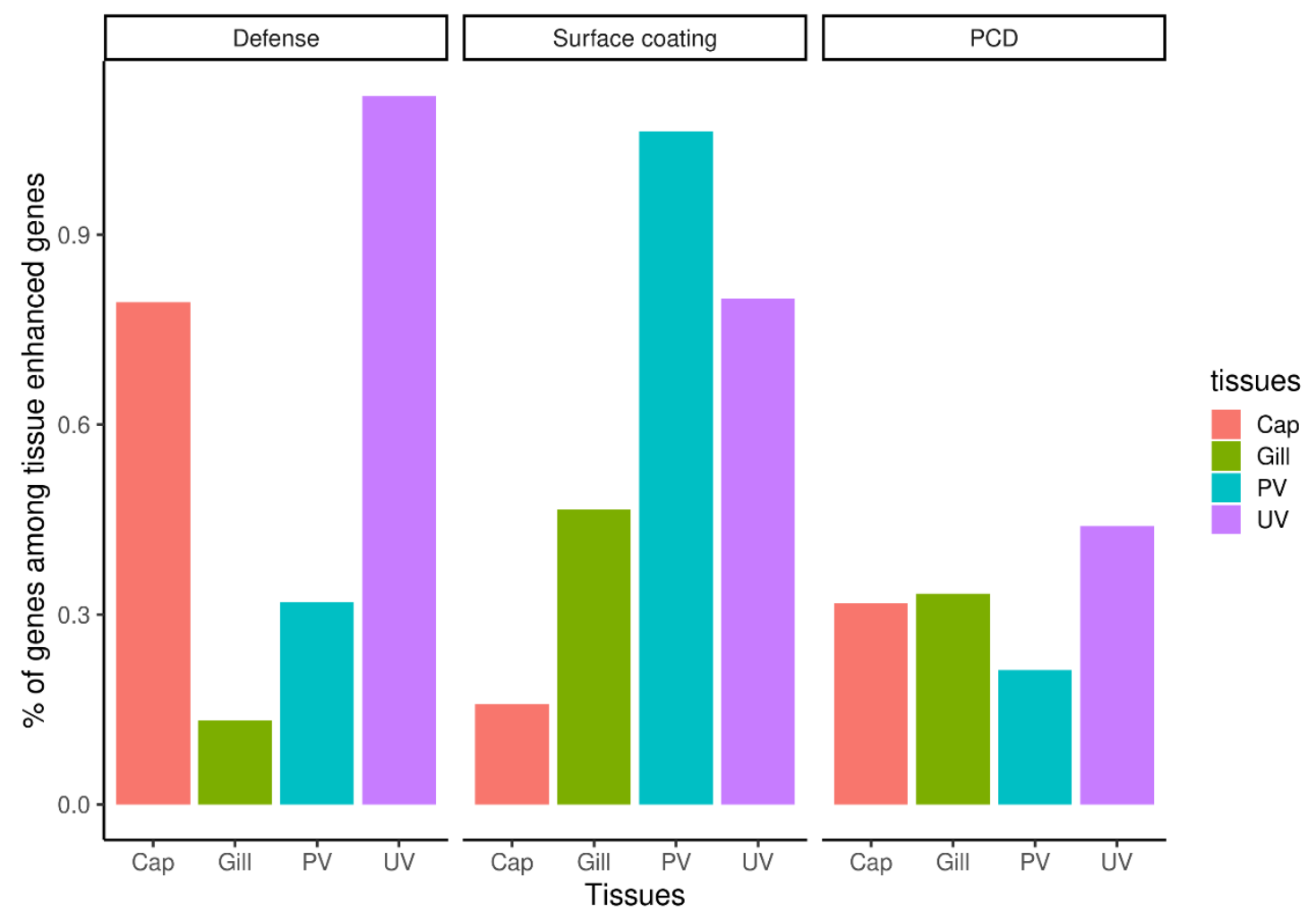

Figure 41. The percentage of genes with a 'Defense', 'Surface coating' or 'PCD' (programmed cell death) functional categories across tissue enhanced genes. $P V=$ Partial veil, $U V=$ Universal veil

Next, we inspected the overall transcription profiles of genes which were identified as tissue enhanced and the member of one of the functional groups mentioned above (Electronic Appendix 7). We found exciting expression patterns that could give us interesting case studies. For example, some genes showed high expression in tissues close to the environment, like primary hyphal knot, universal veil, cap, or upper cap. The gene expression of a ricin b-like lectin (Copci_AmutBmut1_408852) in the primary hyphal knot, the universal veil of all stages, and the cap tissues exceeded the gene expression in other tissues (Figure 42.). In stage 2 primordium, the upper cap tissue showed higher expression than the cap tissue, which is in line that the upper cap is closer to stage 2 primordium's surface than the cap tissue. This pattern could be detected in the case of two galectins, cgl1 (Copci_AmutBmut1_473274) and cgl2 (Copci_AmutBmut1_488611) (Figure 42.). We found 13 tissue enhanced hydrophobin genes from which one (Copci_AmutBmut1_363242) showed high expression in mycelium and in the universal veil, upper cap and gill tissues as well (Figure 42.). Hydrophobins are small hydrophobic 
proteins which could have pivotal role on the cell surface in many functions such as development or pathogeny. Furthermore, we detected four cerato-platanin proteins (CPPs) which were enhanced in the cap and the universal veil (Figure 42.). Interestingly, both cap and universal veil enhanced genes were upregulated in the primary hyphal knot (Copci_AmutBmut1_439071,Copci_AmutBmut1_408843) and in the universal veil of the secondary hyphal knot (Copci_AmutBmut1_439071, Copci_AmutBmut1_408843, Copci_AmutBmut1_522040), indicating that one protein could be highly active throughout the entire development of a tissue. One CPP (Copci_AmutBmut1_544218) exhibited high expression in the universal veil, the upper cap and the mycelium as well (Figure 37.), a pattern we saw in the case of hydrophobins. 


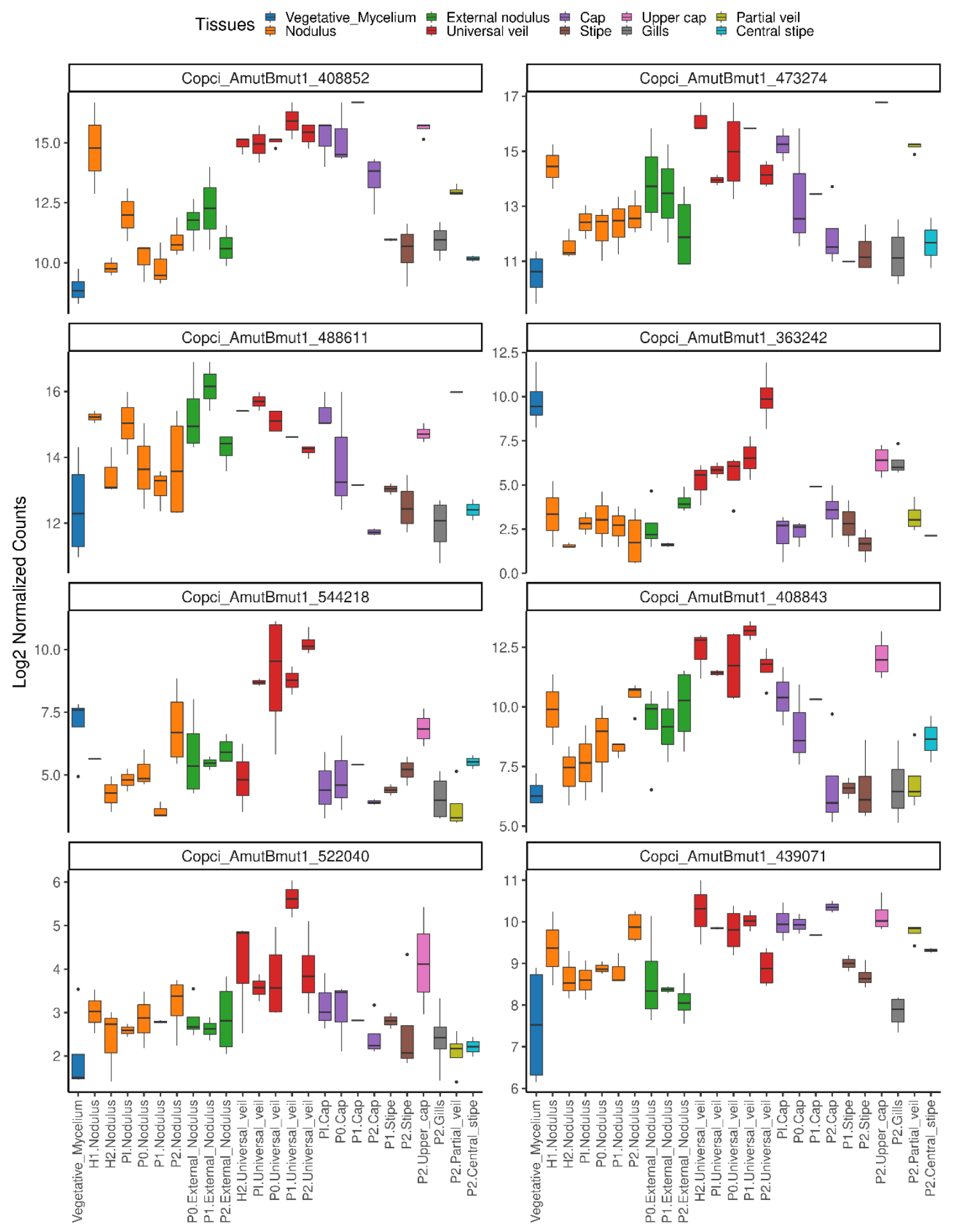

Figure 42. Global gene expression patterns of some tissue enhanced genes. First row. A ricin blike lectin (Copci_AmutBmut1_408852). First and second rows. cgll (Copci_AmutBmut1_473274) and cgl2 (Copci_AmutBmut1_488611) galectins. Second row. A hydrophobin gene (Copci_AmutBmut1_363242). Third and fourth rows. Cerato-platanin genes. 
High expression levels in tissues close to the environment were detected for four wax synthase coding genes as well (Copci_AmutBmut1_244583, Copci_AmutBmut1_478454,Copci_AmutBmut1_372439,Copci_AmutBmut1_490873) indicating that wax esterase could have special role (e.g., surface coating) in these tissues (Figure 43.). We also noticed patterns where the expression value in most of the tissue types was close to 0 except in the universal veil (Copper-dependent lytic polysaccharide monooxygenases (LPMOs, AA9): Copci_AmutBmut1_320571 and lysozyme (GH24): Copci_AmutBmut1_432813, Figure 43.), such genes might be considered as veil-specific marker genes.

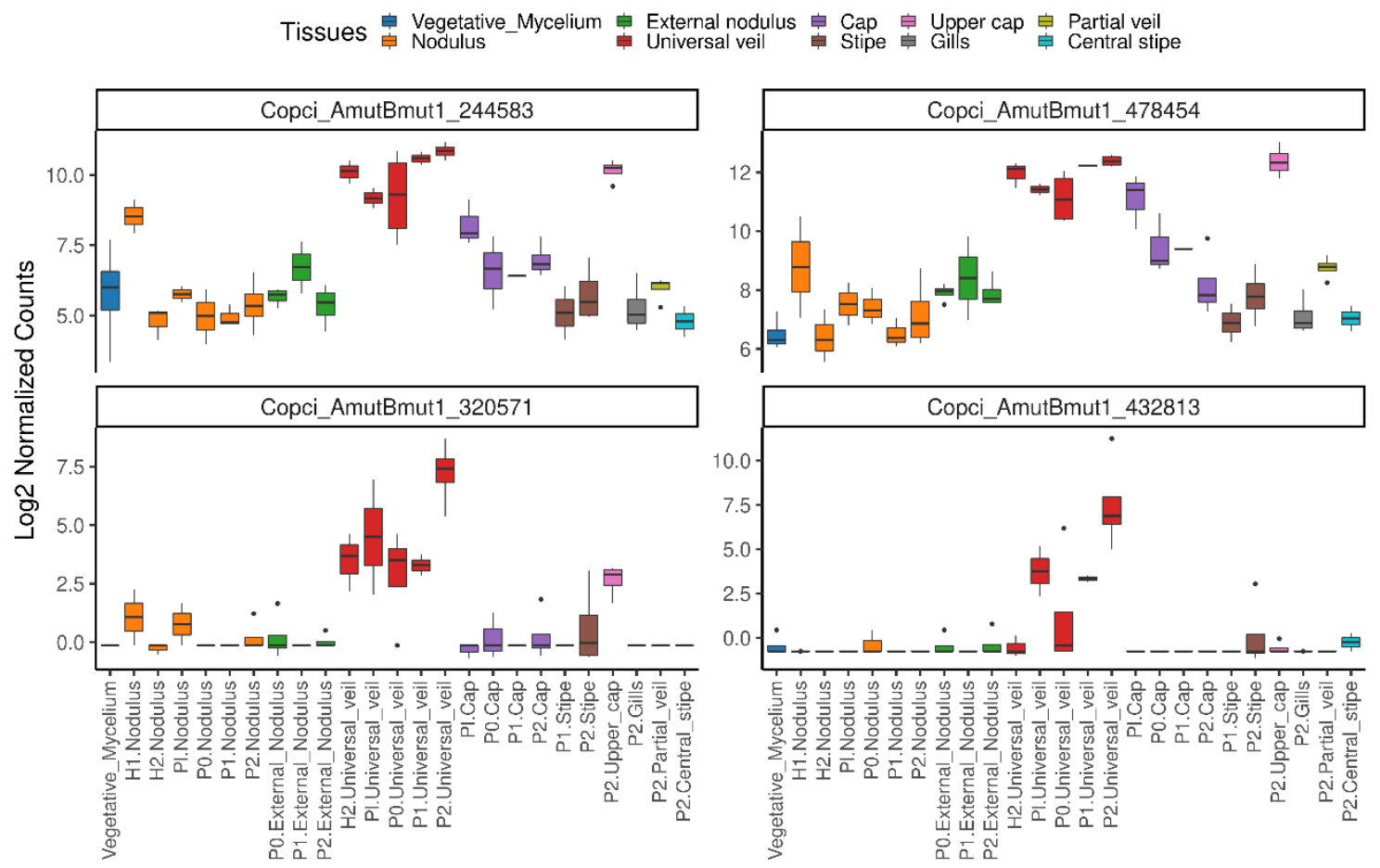

Figure 43. Global gene expression patterns of two wax synthase genes (first row) and potential universal veil marker genes (second row). Copper-dependent lytic polysaccharide monooxygenases (Copci_AmutBmut1_320571) and lysozyme (Copci_AmutBmut1_432813).

We also observed expression patterns dependent on both the developmental stage and tissue types (Figure 44.). In the case of a protein (Copci_AmutBmut1_376687) that belongs to the GH3 family, the encoding gene had the highest expression in the nodulus tissues. Moderate values in the cap and external nodulus and lowest expression could be detected in the universal veil and the primary hyphal knot samples. Within the nodulus, external nodulus and the universal veil tissues a decreasing expression level can be noticed as the development progresses. In contrast to this, the expression of this gene in the cap tissues is increased towards more advanced developmental stages. 


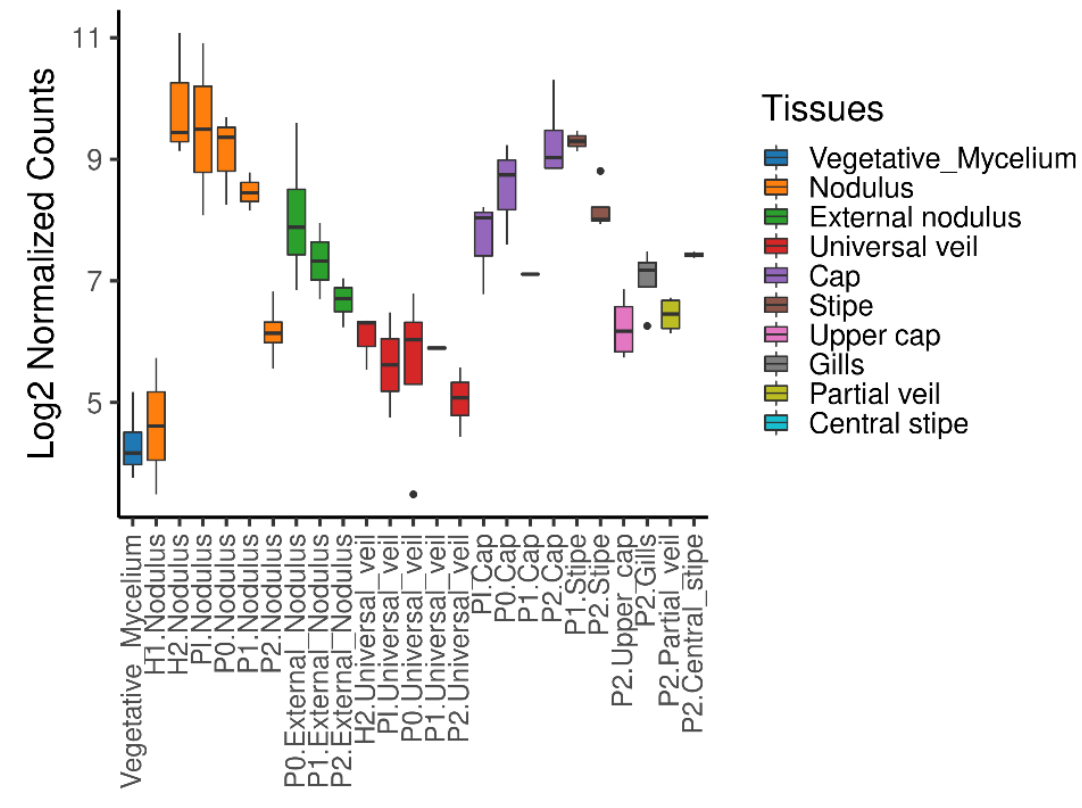

Figure 44. The expression of the Copci_AmutBmut1_376687 gene shows variation across tissue types. It also shows a decreasing tendency in the nodulus, external nodulus, universal veil, and increasing tendency in the cap tissues as the development progresses.

\subsubsection{Genomic phylostratigraphy}

We wanted to examine the evolutionary origin of tissue enhanced genes, therefore, we performed a phylostratigraphic analysis using 441 genomes across fungi. Phylogenetic ages, 'phylostrata' were assigned to each gene based on the last common ancestor of $C$. cinerea and other species identified through RBH analysis. Most of the $C$. cinerea genes originated at or before the MRCA of Ascomycota and Basidiomycota. This node is also known as the MRCA of the Dikarya clade (Figure 45.). Another set of genes emerged in along six nodes of Basidiomycota, between the stem node of Dacrymycetes and the stem node of the Polyporales-Russulales clade. The phylostratigraphic distribution of the four gene sets containing tissue enhanced genes (cap, universal veil, partial veil and lamellae) showed similar patterns to that of all genes. However, enrichment analysis detected age indexes, which were more abundant in one or more gene sets. In all tissue enhanced gene sets except the partial veil, the enrichment of phylostratum 21 was detected (adj. p < 0.05), which represents the MRCA of Asco- and Basidiomycota. Interestingly a relatively young phylostratum, PS9 was enriched among the universal veil enhanced genes. PS9 represents the MRCA of the Marasmioid clade sensu Matheny (Matheny et al., 2006) formed by Physalacriaceae, Schizophyllaceae, Omphalataceae and C. cinerea. In addition to PS21 and PS9, phylostrata 23-25, which 
represent ancient fungal and eukaryotic genes, were enriched in universal veil enhanced genes. Among the lamellae and partial veil enhanced genes, the phylostratum 27 was also enriched, representing the root of the tree.

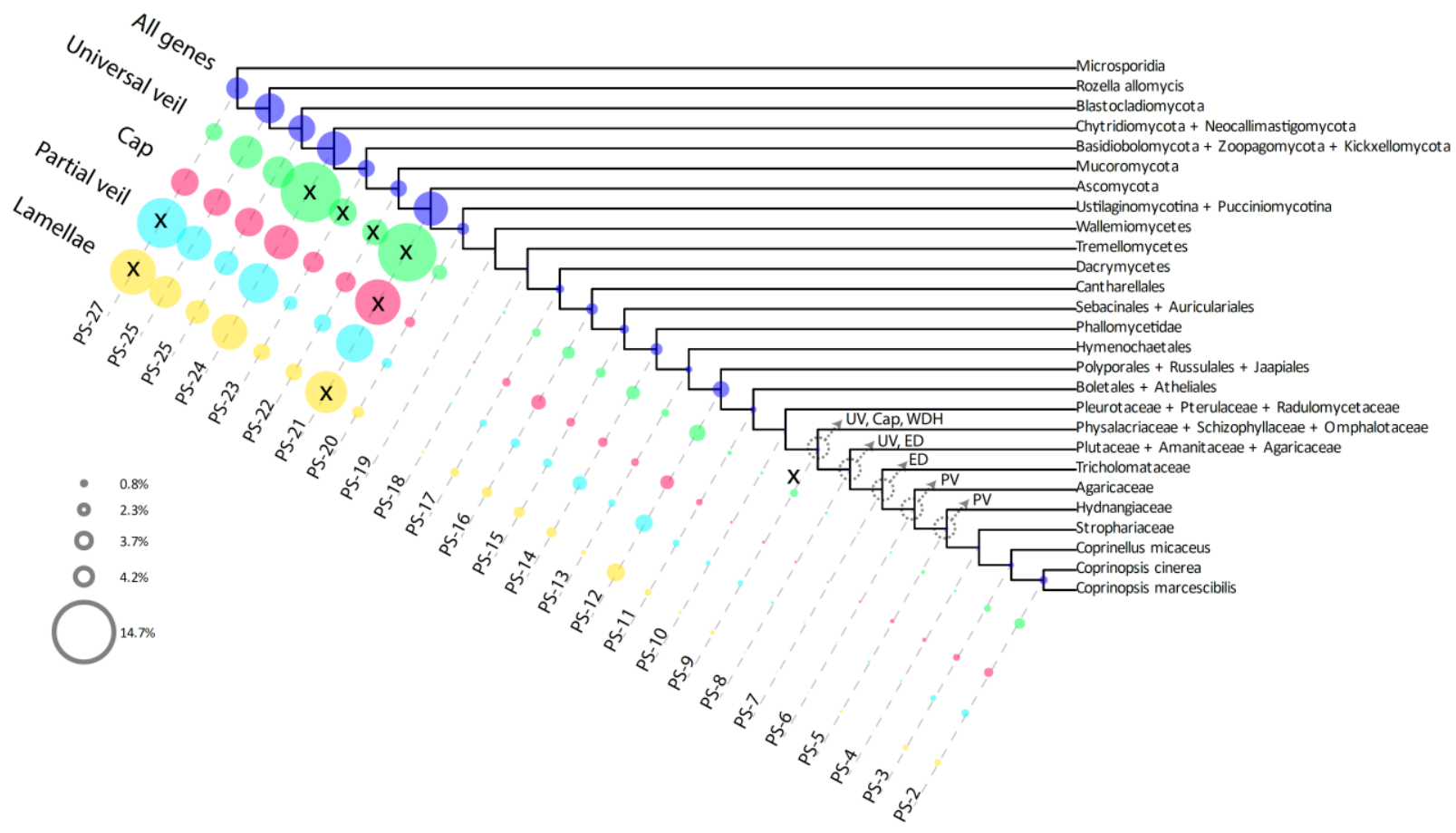

Figure 45. The origin and distribution of tissue enhanced genes along the backbone of Fungi. ' $x$ ' inside the circles depict that the given age index (PS) significantly enriched within the given tissue enhanced gene set(hypergeometric test, adj. $p<0.05)$. Starting from PS9, arrows denote the evolution of universal veil $(U V)$, partial veil $(P V)$, cap, enclosed development $(E D)$ and welldeveloped hymenophore (WDH), based on the ancestral state reconstruction analyses.

We performed maximum parsimony based ancestral state reconstruction (ASR) analysis using ten chronograms of the 5,284-species dataset. We reconstructed the ancestral states of the enclosed development, cap, universal veil, partial veil and increased hymenophore surface area traits. Next, we aligned the phylostratigraphic and ASR results (Figure 45.). We found that the cap and the well-developed hymenophore evolved at PS9 in most of the chronograms ( 7 and 8 out of the 10 trees, respectively). The universal veil probably occurred at PS8 (7 out of 10 trees), but in 2 trees the first occurrence at the backbone mapped to the PS9 node. Interestingly, enclosed development and the partial veil showed up only in the PS8 or PS7 and the PS6 or PS5 nodes, respectively.

Because the PS9 age index enriched among universal veil enhanced genes, we examined their functional annotations (Electronic Appendix 13). Out of the 40 genes, 15 genes had no annotations at all. Three transcription factors (Copci_AmutBmut1_482097, 
Copci_AmutBmut1_419773, Copci_AmutBmut1_498859) and three protein kinases (Serine/threonine PK: Copci_AmutBmut1_432448, Copci_AmutBmut1_478871; fungal type PK: Copci_AmutBmut1_546199) were found among the annotated proteins. We also found two genes with F-box domain (Copci_AmutBmut1_445136, Copci_AmutBmut1_442434). Other genes had functional annotations related to 'ergosterol biosynthesis', 'vesicle transport', 'glycoside hydrolase', or general metabolic and housekeeping functions (for the full list, see Electronic Appendix 13).

Overall, we found that most of the tissue enhanced genes preceded the evolution of main tissue types, and dated to the MRCA of Dikarya or early-diverging fungus and eukaryotic ancestors. However, in the case of the universal veil, a set of genes enriched at the MRCA of the Marasmioid clade sensu Matheny. This coincides with the evolution of the universal veil, cap, well-developed hymenophore and the enclosed development. Finally, this node was dated to the Jurassic period (161.14 Ma +/- 6.75; 5,284-species dataset), where the diversification boost was observed in our macro-evolutionary analysis. 


\section{Discussion}

\subsection{The evolution of complex multicellularity in fungi is incompletely known}

Complex multicellular organisms dominate our present ecosystems and have inevitable roles in ecosystem functioning. However, how the complex multicellularity evolved and reached the complexity level we encounter today is still barely known. Complex multicellularity in animals, plants and algae evolved once (Knoll, 2011) and could be driven by predation as an evolutionary pressure (Rokas, 2008), resulting in numerous genetic innovations (Niklas et al., 2020). Less is known about fungal complex multicellularity, yet, they possess at least eight independent origins (Nagy et al., 2018), three in Mucoromycota, two in Ascomycota and three in Basidiomycota. Complex multicellularity ranges from minute asexual reproductive structures or several meter long rhizomorphs to fruiting bodies with $\sim 20$ cell types, all of these structures are with genetically determined shape and size (Lord and Read, 2011; Kües and NavarroGonzález, 2015).

Fruiting body production is the highest level of morphological complexity that fungi have reached through evolution (Nagy et al., 2018). The largest fungal clade (>25,000 species), which contains almost exclusively fruiting body producing species, is the class Agaricomycetes, also known as mushroom-forming fungi. Despite their ecological (Orwin et al., 2011; Dighton, 2016), economical (Royse et al., 2017; Grimm and Wösten, 2018; Hyde et al., 2019), and medicinal (Sullivan et al., 2006; Gargano et al., 2017) importance less is known about their global evolutionary patterns. So far, it has been shown that the evolution towards the pileate-stipitate mushrooms is favored by species (Hibbett, 2004) and gasteroid forms possess a higher diversification rate in certain clades (Wilson et al., 2011). However, mainly due to the lack of fossil data, our knowledge is limited on global patterns of the evolution of mushroom-forming fungi. The genetic background of fruiting body formation has been examined in many species recently, using bulk RNA-seq methods (Muraguchi et al., 2015; Pöggeler et al., 2018; Krizsán et al., 2019; Merényi et al., 2020). Based on these studies, it starts to be evident that most genes participating in the development of multicellular structures predate the 
evolution of multicellularity in fungal lineages and roots in ancient eukaryotic ancestors (Kiss et al., 2019).

In this study, we attempted to scrutinize the macro-evolution of mushroomforming fungi, where most of the complex multicellular fungal species can be found. We also tested whether morphological innovations could trigger the evolution of Agaricomycetes. Next, by focusing on the model mushroom $C$. cinerea, we wanted to find out whether tissues specific transcriptome can provide a blueprint of morphological traits that contributed to the evolutionary success of mushroom species.

\subsection{Reconstructing the evolutionary relationships within the Agaricomycetes based on 5,284 species}

To examine macro-evolutionary patterns through the evolution of mushroomforming fungi, we inferred 245 phylogenetic trees consisting of 5,284 species of Agaricomycetes, Dacrymecetes and Tremellomycetes. Our phylogeny was constructed from three genomic loci (tef1- $\alpha$, nrLSU, and rpb2), and a genomic backbone that consists of 104 species. We found that the 245 trees cover a sufficiently plausible set of topologies based on Robinson-Foulds distances. The main relationships among lineages were consistent with other studies (Hibbett, 2006; Hibbett et al., 2014). Our phylogeny recovered the early divergence of Cantharellales, Sebacinales, Auriculariales and the monophyly of the Phallomycetidae subclass, which consists of the orders Geastrales, Gomphales, Phallales and Hysterangiales (Floudas et al., 2012; Hibbett et al., 2014; Kohler et al., 2015). Our phylogeny supports the grouping of Agaricales, Boletales, Amylocorticiales, Atheliales and Lepidostromatales into the subclass Agaricomycetidae. However, we found that Russulales was the sister group of Polyporales in contrast to the majority of the studies where Russulales was recovered as the sister clade of the Agaricomycetidae (Hibbett, 2006; Floudas et al., 2012; Doré et al., 2015; Zhao et al., 2017).

We inferred ten time-calibrated trees using a two-step Bayesian molecular clock dating procedure on 5,284 species. Before constraining nodes through the molecular clock dating using eight fossil calibration points, we performed a fossil-cross validation procedure. The cross-validation analysis showed that none of the chosen fossils were in conflict with each other. The crown node of most of the main orders was dated to the 
Jurassic period, including Cantharellales (mean: 184 +/- $44 \mathrm{Ma}$ ), Hymenochaetales (mean: 152 +/- $14 \mathrm{Ma}$ ), Russulales (mean 147 +/- $14 \mathrm{Ma}$ ) and Agaricales (mean: 172 +/$8 \mathrm{Ma}$ ). The crown nodes of the two subclasses, Phallomycetidae (Gomphales, Phallales, Geastrales, Hysterangiales) and Agaricomycetidae (Agaricales, Boletales, Amylocorticiales, Jaapiales, Atheliales) were also dated to the Jurassic (174 +/-18 Ma and $182+/-9 \mathrm{Ma}$, respectively). By comparing the age estimates of clades with that of recent molecular clock studies (Floudas et al., 2012; Doré et al., 2015; Krah et al., 2018; Lutzoni et al., 2018), we found that our time inference was comparably older, except the age estimates of Sánchez-García et al. (Sánchez-García et al., 2020). For example, the emergence of Agaricales was dated to 90 and 108 Ma by Floudas et al. (Floudas et al., 2012) and Kohler et al. (Kohler et al., 2015), respectively, while our mean estimated ages ranged between 160-182 Ma depending on the tree analyzed. These differences could be caused by taxon sampling density or the calibration schemes used in different studies.

To explain the difference in age estimates, we inferred a time-calibrated phylogenomic tree and performed a sensitivity analysis. First, we replicated the calibration scheme used in the study of Kohler et al. 2015. Then, we modified the calibration scheme of Kohler et al. 2015 by placing fossil constraints on the crown node of Suillaceae and Marasmioid clade. Constraining these crown nodes was possible for us due to the intensive sequencing of fungal genomes in recent years (Grigoriev et al., 2014; Lewin et al., 2018). We found that the most profound effect on the inferred ages was exerted by the position of fossil calibration points and, to a lower extent, the number of available genomes, the type of analyses (non-parametric, Bayesian analyses), or the type of the dataset (phylogenomic or phylogenetic trees).

Overall, we think our time estimates are robust and reliable, given the fossil-cross validation, the sensitivity analyses, and the similar age estimates of different methods. Furthermore, the older age estimates of the clades of Agaricomycetes is in concordance with a recent finding of the oldest fungal fossils representing filamentous forms with chitin content (Bonneville et al., 2020). This fossilized fungal filaments (Mbuji-Mayi Supergroup) pushed the oldest known fungi by at least 250 Ma years back, suggesting that fungal clades could be older than it has been believed so far. 


\subsection{Macro-evolutionary patterns among mushroom-forming fungi}

\subsubsection{Diversification rate increase in the Jurassic period}

We analyzed ten time-calibrated trees using BAMM to uncover global speciation rate patterns through the evolution of mushroom-forming fungi. We found that the diversification rate abruptly increased in the early Jurassic period ( $180 \mathrm{Ma})$ and kept gradually increasing until the present time. This period coincides with the breakup of Pangea, which resulted in larger coastlines areas. During this geological process, the arid climate of the Triassic period changed to a humid and warm climate characteristic to the Jurassic period (National Research Council, 1982). Based on the environmental preferences of extant species, we can assume that the climate of the Jurassic was optimal for mushrooms, favoring to colonize new regions and environments by fungal species. Based on the limited literature data, the abruptly increased diversification in the Jurassic seems to be a unique pattern for mushrooms among fungal taxa. A recent study (Nelsen et al., 2020) examined the macro-evolution of lichen-forming fungi of the class Lecanoromyces (Ascomycota) based on a megaphylogeny of 3,373 species. They did not find a boost in diversification rate in the Jurassic, instead of a gradual increase of speciation rate, which was more pronounced starting from the mid-Cretaceous. However, it was shown that other organisms, such as gymnosperms or herbivorous beetles, went through an expansion in the Jurassic period (Niklas et al., 1983; McKenna et al., 2009). Therefore, the evolutionary pattern we observed in Agaricomycetes is not a unique phenomenon among organisms. The increase of the diversification rate stopped at the end of the Cenozoic period, which could be explained by many causes such as protracted speciation, niche differentiation, geographic factors (Moen and Morlon, 2014). To disentangle to possible causes of the diversification slowdown more data and deeper analyses would be needed in the future.

\subsubsection{Mass extinction events could follow different patterns than in other organisms}

Fossil data is one of the primary sources to examine extinction events through evolution (Jablonski, 1994). However, fungi lack abundant and statistically evaluable macro-fossil data. Therefore, we examined the variation in the inferred extinction rates through the evolution of mushroom-forming fungi. Based on BAMM analysis, we 
observed an extinction rate peak at the Jurassic-Cretaceous (J-K) boundary (Figure 7.). To further disentangle this extinction signal in our data we performed macro-evolutionary analyses using an episodic stochastic-branching process model (CoMET) (Höhna et al., 2016; May et al., 2016). We found that models implementing mass extinction events were superior over models without extinction events. Furthermore, using rjMCMC analyses, we found that a mass extinction could occur with high probability at the J-K boundary. Putting together the extinction rate analyses mentioned above, we conclude that the onset of increased diversification in the Jurassic might have been followed by a mass extinction event at the J-K boundary. Our finding is supported by the fact that mass extinctions occurred in multiple ancient groups at this period (Kauffman and Hart, 1996), including both marine and non-marine organisms but not among plant lineages (Tennant et al., 2017). Interestingly, extinction was prevalent among specialized organisms and in lowlatitude shallow coastline areas (Tennant et al., 2016).

We did not find any signal for extinction at the Cretaceous-Paleogene (K-Pg) boundary (Jablonski, 1994). In this period, most of the main groups suffered severe extinction, including birds, mammals, reptiles, insects, marine organisms and plants (Novacek, 1999; Longrich et al., 2011; Henehan et al., 2019) due to extreme environmental changes, among others the impact of an asteroid (Wilf et al., 2003; Schulte et al., 2010; Sprain et al., 2019). In this context, our finding is surprising, but it is supported by fossil data. Vajda and McLoughlin (Vajda and McLoughlin, 2004) examined a coal seam in New Zealand dated to the K-Pg boundary. They found a fungal microfossil spike between the Late Cretaceous and the early Paleocene floral fossils. It was hypothesized that after vast deforestation (Vajda, 2001), a large amount of biomass buffered the environmental impacts on fungi and favored fungal proliferation. A similar pattern was observed at the Permian-Triassic boundary (Benton and Twitchett, 2003), but not at the J-K boundary. This and the fact that plants did not suffer an extinction at the J$\mathrm{K}$ boundary may imply the importance of dead plant material in surviving critical periods by fungi.

\subsubsection{Increased diversification rate is in concordance with the evolution of complex morphologies}

We inferred shifts in speciation and extinction rates through time and across lineages of mushroom-forming fungi using BAMM analysis. We detected 87 clades with 
taxonomically congruent core shifts across the ten chronograms analyzed. Out of the 87 clades, 57 consisted of species with a cap, implying the importance of the pileate fruiting body type. Therefore, we reconstructed the ancient character states of the main fruiting body types and found that the evolution of complex morphologies coincides with the diversification burst in the Jurassic (Figure 10.). Moreover, the proportion of pileatestipitate morphology among mushroom-forming fungal species increased through time and has a prevalence among extant taxa.

It seems that the global diversification rate is more affected by morphological innovations than ecological factors. Sánchez-García et al. (Sánchez-García et al., 2020) examined both the effect of main fruiting body types and nutritional modes (brown rot and white rot wood decaying-fungi, other saprotrophs, pathogens and ectomycorrhizal species) on the speciation rates of mushroom-forming fungal species. They found that nutritional modes had no effect on the diversification rates, but species with pileatestipitate morphology had significantly higher diversification rates than species with other morphologies. Nelsen et al. (Nelsen et al., 2020) did not find any effect of different symbiont partners on the diversification of Lecanoromycetes lichen-forming fungi. However, they revealed a marginally positive effect of thallus morphology on the speciation rate.

Apart from the morphological traits, the elevated diversification of mushroomforming fungi may coincide with the radiation of gymnosperms in the Jurassic (Niklas et al., 1983). This agrees with the MRCA of most of the orders could have associated with gymnosperms as symbiotic hosts or substrate for wood decay (Varga et al., 2019). This pattern was observed in the radiation of herbivorous beetles (McKenna et al., 2009). In a smaller scale, the effect of nutritional modes was indicated in Laccaria (Wilson et al., 2017), Tricholoma, Entoloma (Sánchez-Garcia et al., 2017), Boletaceae (Sato and Toju, 2019) and among brown and white rot fungi (Krah et al., 2018). However, this study and the study of Sánchez-García et al. (Sánchez-García et al., 2020) suggest that the global evolutionary patterns could be driven by morphological innovations through Agaricomycetes evolution. 


\subsubsection{Five morphological traits with a positive effect on the diversification of mushroom-forming fungi}

Based on the results and studies described above, morphological novelties could be the main drivers of global patterns of mushroom evolution. Therefore, we examined five morphological traits that could serve as a key innovation among Agaricomycetes: cap formation, the increased surface area of hymenophore, enclosed development, the presence of universal veil, and that of partial veil as protecting sheaths (Figure 3., Table 2.). Four out of the five traits examined can be associated with the protection and nursing of the developing fruiting body and the spore-bearing structure (hymenophore). The cap can supply a protecting environment for spore production by shielding the hymenophore from above. Additionally, many species can surround the hymenophore or the whole fruiting body by specialized tissues and sheaths lasting until the development of the mature (enclosed development) or young (semi-enclosed development) fruiting body. Enclosed development includes gasteroid mushrooms where spore production entirely takes place inside the fruiting body, and it lacks active spore release (Thiers, 1984). Other species evolved specialized tissues to cover up the fruiting body (universal veil) or only the hymenophore (partial veil). However, the veils rupture at the latest after the spores have matured, allowing the spores to be actively dispersed. Not only protection can aid the reproductive success of an organism but effective spore dispersal strategies too. For example, increased hymenophore surface area can provide more spores relative to the mass of the fruiting body. Therefore, we examined smooth, weakly developed and welldeveloped hymenophores in our macro-evolutionary analyses.

Using Mk, BiSSE and MuSSE models, we examined the evolution of the five traits and the state-dependent diversification of species. Cap production, enclosed development, the presence of universal veil, or partial veil all significantly increased the diversification of species through the evolution of mushroom-forming fungi. All these traits can support and protect the developing spores. Cap can give a protection from the above impacts, such as rain or ultraviolet light and accordingly many species produce mucinous extracellular materials and pigments (Velíšek and Cejpek, 2011). Moreover, presence of pigments could affect the diversification of species. It was shown that anthraquinone, a secondary metabolite that protects against ultraviolet light increased the diversification rate of Teloschistales lineages (Lecanoromycetes, Ascomycota) relative to lineages without this metabolite. (Gaya et al., 2015). Apart from the shielding effect of 
the cap, it can also aid spore dispersal (Dressaire et al., 2016), promoting diversification. We found higher transition rates towards pileate states than the reverse directions, which also supported the evolutionary importance of the cap. Our results are in line with other studies that showed higher gains of pileate-stipitate morphologies and higher diversification of lineages with pileate-stipitate morphologies than other lineages (Hibbett, 2004; Sánchez-García et al., 2020).

The protection of spore development can be further facilitated by providing a fully closed environment through the course of ontogeny. We showed that lineages with semienclosed and enclosed development had a higher diversification rate than lineages with open development. Strategies of creating a safe environment for progeny development, including viviparity, is a widespread feature among both vertebrate and invertebrate lineages (Blackburn, 2015; Ostrovsky et al., 2016). Furthermore, viviparity is also known from some plant lineages, where the growth of the new individual starts on the parental sporophyte (Elmqvist and Cox, 1996). It was shown that viviparous squamate species had higher speciation rate than oviparous ones (Pyron and Burbrink, 2014) and viviparous fishes from Cyprinodontiformes clade had higher diversification rate than other species (Helmstetter et al., 2016). Furthermore, phylogenetic studies of Ascomycota put to basal position of species with open fruiting body (apothecia) and to derived position of species with enclosed fruiting body (perithecia) (Liu and Hall, 2004). Among lichen-forming fungi of the Lecanoromycetes clade, several independent occurrences of enclosed (angiocarp) fruiting bodies were shown (Schmitt et al., 2009). In conclusion, the studies on Ascomycota further support the idea of a general evolutionary trend towards enclosed development in nature.

We also tested the impact of the universal veil and the partial veil on the diversification of mushroom-forming fungi. We found slight but significantly bigger diversification rate of species with either of the veils than species without any of the veils. These tissue layers cover the outer surface of the fruiting bodies, forming a barrier between inner tissues and the environment. However, these protective tissues form not just a physical barrier against environmental impacts but produce secondary metabolites, peptides and proteins against bacteria or fungivore animals (Jaeger and Spiteller, 2010; Künzler, 2018). For example, lectins, which are effective nematotoxic and entomotoxic proteins (Bleuler-Martínez et al., 2011; Sabotič et al., 2016), have high expression in the velum of the cap during the development of Coprinopsis cinerea (Boulianne et al., 2000). 
Furthermore, annulus (ring on the stem), cortina (spider web like structure on the cap margin) and other special structures could be formed from the veil remnants after the breakup of the veils. It was hypothesized that these structures could serve as an obstacle against insects or can give a better aerodynamic feature for spore release (Halbwachs et al., 2016).

Besides protecting the developing progenies, producing more descendants can also increase the reproductive success of organisms. In line with this, it was hypothesized that the driving force of fruiting body evolution is effective spore dispersal (Hibbett and Binder, 2002). We revealed that species with well-developed hymenophores (e.g., pores, lamellae) had significantly higher diversification rates than species with smooth or weakly structured hymenophores. Spore production, like other airborne dispersal strategies (e.g., dispersal of plant seed by wind), adapted to propagate an enormous number of spores (Halbwachs and Bässler, 2015), explaining why increased hymenophore surface area can positively affect the evolution of species. However, additional factors such as lamellae positioning (Fischer and Money, 2010), protection against predators (Nakamori and Suzuki, 2007), keeping high humidity for ballistospore release (Halbwachs and Bässler, 2015) or air dynamics (Dressaire et al., 2016) can strongly affect and shape the hymenophore of mushrooms, making the picture more complex.

Semi-enclosed development, weakly structured hymenophore, the presence of universal veil or partial veil behaved as labile states in our analyses. These states, except weakly structured hymenophore, significantly increased the diversification of species. However, the inferred transition rates showed frequent loss of these states during the evolution. The transition rates from semi-enclosed development towards open or enclosed development were higher than the corresponding reverse directions. At the same time, species with semi-enclosed development had the highest diversification rate. Likewise, the transition rates from veiled states (universal or partial veils) towards non-veiled states were higher than the reverse directions. The transition rates from weakly structured hymenophore towards smooth or well-developed hymenophore were also higher than the corresponding reverse directions. By keeping in mind that this pattern could be caused by the discretization of continuous traits because a discrete character does not represent the true diversity and complexity of the trait (Parins-Fukuchi, 2018), the interpretation of labile morphological traits is also worth considering. Having a labile morphological 
character is not a rare phenomenon. It was discovered that the body morphology of male fig wasps (Jousselin et al., 2004), the behavioral systems of ants (Robson et al., 2015), the floral development of legume lineages (Bruneau et al., 2014), and even the viviparity/oviparity of different populations within a species (Velo-Antón et al., 2012) can easily and frequently alter through time. Pyron et al. (Pyron and Burbrink, 2014) found that parity mode can be a labile trait and simply respond to ecological conditions (e.g., temperature), highlighting the importance of the environmental conditions in morphological evolution.

\subsection{Developing a low-input tissue-specific RNA-seq workflow}

\subsubsection{Dissecting the early development of C. cinerea}

As we described above, certain morphological traits could positively affect the diversification of mushroom-forming fungi, thus we were curious about what genes are responsible for mushroom development and tissue formation. Therefore, we decided to examine the tissue-specific transcriptome through the early development of $C$. cinerea. Before RNA-seq, we wanted to discern better and describe the histology of the early development of $C$. cinerea (Figure 25.). Even though the development of this species is one of the best-studied ontogenies among fungi (Moore et al., 2011; Kües and NavarroGonzález, 2015), less is known about the beginning of the process. We reasoned that the early stages are most informative as to the general principles of development because at the beginning of the ontogeny (stage 1 primordium sensu Kües) the main tissues (nodulus, cap, stipe) have already been produced (Kües, 2000). Therefore, we carried out more than 200 histological examinations using cryo-sectioning and light microscopy. We checked the dependency of different primordium occurrences on the time of the day but did not find any association between them. However, we observed two intermediate stages (primordium initial and stage 0 primordium) between the secondary hyphal knot and the stage 1 primordium, which were not mentioned in the literature except in the study of Matthews and Niederpruem (Matthews and Niederpruem, 1973). They described a developmental stage of $0.5 \mathrm{~mm}$ in diameter with an enhanced cellular activity at the apex of the primordium. This description and the photograph of the primordium (Figure 2.A in Matthews \& Niederpruem, 1973) resembles the primordium initial stage observed in our study. Kües and Navarro-González (Kües and Navarro-González, 2015) note that some of the histological descriptions can represent aborted primordia. However, the 
transcriptome profile of the cap-initial tissue in the primordium initial stage shows a close relationship with that of cap tissue in stage 2 primordium (Figure 33.). The close relatedness of cap tissues indicates a stable genetic program from the first sign of its formation to the well-developed cap of the stage 2 primordium. We also delineated the stage 0 primordium, where a small globular cap tissue develops at the apex of the primordium, but no clear differentiation of stipe tissues could be observed. In contrast, the miniature pileate-stipitate mushroom shape can be recognized in the cross-sections of stage 1 primordium.

Finally, we examined six developmental stages plus the vegetative mycelium ranging from the $0.15 \mathrm{~mm}$ sized primary hyphal knot to the stage 2 primordium with $\sim 1.9$ $\mathrm{mm}$ in height. We also included nine morphologically well distinguishable tissue types in our study. Additionally, to the well-described tissue types in the literature (cap, nodulus/basal plectenchyma, lamellae, universal veil, partial veil, stipe) (Moore et al., 1979; Kües, 2000; Clémencon, 2012; Kües and Navarro-González, 2015), we defined three extra tissue types. The external nodulus first occurs at stage 1 primordium between the nodulus and the universal veil. Probably it is a transitional tissue between the inner and outer layers. It contains not as inflated and loosely packed hyphae as the universal veil but shows less compacted structure than the nodulus. The upper cap (stage 2 primordium) could also be an intermediate tissue between the cap and the universal veil showing a distinct histological image. It has already been noted that the cap and universal veil form a continuous transition (Reijnders, 1979), therefore, it is reasonable to divide this region into smaller parts. Furthermore, we distinguished the elongated and loosely structured stipe at the center and middle of the stage 2 primordium, here is termed 'central stipe'. We assigned the term 'stipe tissue' in stage 2 primordium to a central tissue below the cap and lamellae, which still showed compact structure as in earlier stages.

\subsubsection{Low-input tissue-specific RNA-seq workflow}

We worked out a procedure to isolate groups of cells (tissues) from cryo-sections of the early developmental stages of $C$. cinerea using laser-capture microdissection (LCM). We used traditional techniques to perform cryo-sectioning but applied RNA protecting approaches at every step. Paraffine-based methods are the most common techniques for RNA-seq coupled with LCM because they can generally give more histological details than cryo-sections (Belmonte et al., 2013; Hacquard et al., 2013; 
Thakare et al., 2014; Khan, D. et al., 2015; Limpens, 2020). However, paraffine based techniques require long incubation times and big temperature fluctuances, which can strongly affect RNA integrity (Botling et al., 2009). In contrast, applying low temperature throughout tissue preparation can aid the preservation of RNA integrity (Bussolati et al., 2011). Consequently, high RNA quality could be reached in our experiment by using cryo-sectioning methods. Furthermore, by carefully executing sample preparation coupled with sucrose infiltration, we could obtain sufficiently detailed histology for identifying main tissue types of $C$. cinerea.

Cell disruption in a minute volume could be a hurdle that had to be overcome to reach a proper amount of RNA for sequencing. This is particularly the case for fungal specimens where enough RNA could be reached only by increasing the amount of tissue catapulted into one sample. For example, 5,000 - 10,000 arbuscular mycorrhiza colonized cortical root cells had to be harvested to perform RNA-seq (Gaude et al., 2012). Similarly, 100-300 protoperithecia ( $\sim 20 \mu \mathrm{m}$ in diameter) were isolated per sample (Teichert et al., 2012) or 80 sections ( $~ 100 \mu \mathrm{m}^{2}$ area/section) of ectomycorrhizal root tip were pooled into one sample (Hacquard et al., 2013) to perform transcriptomic analyses. We tried both enzymatic and mechanical cell disruption methods, but we found that only a heat shock procedure was compatible with our workflow. Still, $5-74$ sections $\left(\sim 0.07-10 \mathrm{~mm}^{2}\right.$ total area) per sample were enough to reach a sufficient amount of RNA $(0.14-17 \mathrm{ng} / \mu \mathrm{l})$. In contrast to fungi, the application of cell disruption methods on plant tissues with cell wall is more advanced. The best example for this, is that the Drop-seq method (Macosko et al., 2015) could be applied on Arabidopsis root by protoplasting the whole tissue and achieving RNA expression profiles of >12,000 individual cells (Shulse et al., 2019).

\subsection{Transition from vegetative mycelium into hyphal knot involved active changes in the expression of genes related to hyphal growth patterns and cell wall remodeling}

The fruiting body development of most of the mushroom-forming fungi starts by producing a primary hyphal knot (Clémencon, 2012). This structure could have a critical role in evolution because it is the "turntable of the development", and not just fruiting body but sclerotium and black stipe (an elongated structure produced in the dark) can further be progressed depending on biotic and abiotic factors (Clémencon, 2012; Kües and Navarro-González, 2015). Forming the secondary hyphal knot with a compact inner 
and a loosely structured outer layer is the first determination towards fruiting body development (Kües, 2000). Therefore, we speculated that we could have a better understanding on the mechanisms underlying mushroom development by examining the changes at the transcriptome level during the transition from vegetative mycelium into hyphal knots.

We found 1,293 - 2,590 genes which were upregulated in either the primary hyphal knot, the nodulus or the universal veil of the secondary hyphal knot by using the vegetative mycelium as a reference. Based on functional enrichment analysis, we found significant enrichment of cell wall remodeling, DNA replication related GO and KOG terms (Electronic Appendix 6). In the literature, it is well known that the growth pattern of the mycelium is changed to form a multicellular structure (Moore et al., 2011; Kües and Navarro-González, 2015). Therefore, we hypothesized that the expression profile of genes related to cell division (cell cycle control, mitosis) and hyphal growth pattern (branching, septation, anastomosis) was changed through the transition from simple (vegetative mycelium) to complex multicellularity (hyphal knots). By examining these functional categories, we assumed that genes having a role in the evolution of complex multicellularity could be detected. We found that 161 genes upregulated in the first two developmental stages were associated with cell division and hyphal growth according to our manually curated functional annotation database (Electronic Appendix 6-7.). Moreover, the number of cell division and hyphal growth related upregulated genes were significantly increased in the secondary hyphal knot relative to the primary hyphal knot (Figure 34.).

Apart from the general mechanisms of cell division described in yeasts ( $\mathrm{Li}$ and Murray, 1991; King et al., 1994; Scholey et al., 2000), our knowledge on cell cycle control and mitosis during the early development of $C$. cinerea is limited. By using a genesilencing method, it was found that a phosphatidylinositol-3-kinase-related kinase (Atr1) and a serine-threonine kinase (Chk1) play a role in the fruiting body and sclerotia formation (de Sena-Tomás et al., 2013), while their primary function is to participate in DNA damage response pathways. Furthermore, they found that the hyphal knot stage can develop in Atr1 or Chk1 silenced strains, but further development was impaired. We found that the Atr1 was significantly upregulated in the nodulus tissue of the secondary hyphal knot (Figure 35.) but was not expressed differentially in the primary hyphal knot. This pattern may explain why it was found that the primary hyphal knot can still develop 
when this gene was silenced (de Sena-Tomás et al., 2013). A common pattern in the expression profiles of the Atr1 and Chk1 genes was that the expression in universal veil tissues was generally low. This pattern could be detected in other cell division related genes such as a S. cerevisiae Prp19 gene ortholog (Chanarat and Sträßer, 2013), S. cerevisiae MAD2 gene ortholog, or a gene with OB-fold motif (Figure 35).

We also detected genes associated with hyphal anastomosis (Figure 35.): ortholog of rac-1 in Neurospora crassa and two genes are part of the striatin-interacting phosphatases and kinases (STRIPAK) complex (Bloemendal et al., 2012). The rac-1 gene is part of the MAP kinase pathway and could be responsible for cell-to-cell communication through the process of hyphal fusion (Fu et al., 2011). The proteins of the STRIPAK complex are highly conserved among eukaryotic organisms and have diverse functions (Kück et al., 2016). In mammals, the STRIPAK complex plays a role in cell migration, cytoskeleton organization, cell size and morphology control, while in fungi, they found its connection with hyphal fusion and sexual development (Kück et al., 2016). In our analyses the PP2A catalytic subunit upregulated in the universal veil, while the SLAMP membrane anchoring subunit was upregulated in the nodulus. We observed no upregulation of hyphal anastomosis related genes in the primary hyphal knot indicating that hyphal fusion is not as frequent in the first stage than in later stages.

Cell morphology can also be modified by altering the cell wall composition and structure (Kamada and Tsuru, 1993), so cells can easily take different shapes. It was shown that CAZymes could play a role in cell wall remodeling (Zhou et al., 2015; Zhang et al., 2016), and many CAZy families went through expansion during the evolution of mushroom-forming fungi (Krizsán et al., 2019). 75 genes were determined as CAZymes and upregulated in the primary and secondary hyphal knots. Many of them were canonical cell wall remodeling genes such as chitinases (GH18) or endo-1,3- $\beta$-glucanases (GH16). However, 13 copper-containing lytic polysaccharide mono-oxygenases (LPMOs, AA9 family) (Hemsworth et al., 2013) were also significantly upregulated in the primary and secondary hyphal knots (Figure 36.). The members of the AA9 (auxiliary activities (AA) 9 family) mostly participated in cellulose decomposition, but activity with chitin was also shown (Agger et al., 2014). Moreover, the developmental regulation of AA9 genes was also detected in Schizophyllum commune (Almási et al., 2019), Ganoderma lucidum (Zhou et al., 2018), Coprinopsis cinerea, Phanerochaete chrysosporium, Lentinus tigrinus, Rickenella mellea and Armillaria ostoyae (Krizsán et al., 2019). We saw 
variations and alternating patterns in the expression profiles of AA9 genes along different tissue types as well (Figure 36.). Overall, we found an outstanding activity of LPMOs in formation of hyphal knot and tissues, thus more detailed studies are needed to better understand the role of these enzymes in fruiting body development.

Not only the structure but also the thickness of the cell wall is changing through primordial ontogeny. Both the thickness of the cell wall (Kamada and Tsuru, 1993) and the beta-glucan content of the hyphal knots were increased relative to the mycelium, which was accompanied by the upregulation of genes related to beta-glucan metabolism (Xie et al., 2020). We found 16 genes related with beta-glucan metabolism and the same number of beta-glucan anabolic and catabolic genes were upregulated in the primary hyphal knot and the nodulus of the secondary hyphal knot. This finding suggests that the cell wall is continuously modified in the hyphal knots. However, in the universal veil of the secondary hyphal knot, twice as many beta-glucan catabolic than anabolic related genes were upregulated (Appendix 3). This pattern may be in concordance with the large inflated morphology of cells in the universal veil. (Reijnders, 1979; Kües, 2000).

Changes in carbohydrate metabolism during the transition from vegetative mycelium to the fruiting body in $C$. cinerea is a relatively well-described phenomenon (Matthews and Niederpruem, 1973; Brunt and Moore, 1989; Krizsán et al., 2019; Xie et al., 2020). We mainly focused on the gene expression changes related to morphogenesis, yet our data show similarities with previous studies on carbohydrate metabolism. It was previously shown that the glycogen content of the hyphal knot was half that of the vegetative mycelium (approximately $400 \mathrm{mg} / \mathrm{g}$ dry mass to $200 \mathrm{mg} / \mathrm{g}$ dry mass) which was in concordance with gene expression changes (Xie et al., 2020). Similarly, we found the upregulation of a glucoamylase (Copci_AmutBmut_473268) in the primary hyphal knot, the universal veil and the nodulus of the secondary hyphal knot. Parallel to glycogen catabolism, genes with a putative function in glycogen anabolism were upregulated in the universal veil and the nodulus as well. A glycogen branching enzyme (GH13, Copci_AmutBmut_365699) was upregulated in the universal veil, which plays a role in glycogen synthesis. We also found the upregulation of a gene (Copci_AmutBmut_463106) in the nodulus, encoding a serine/threonine protein phosphatase whose ortholog (PPG1) is required for glycogen accumulation in $S$. cerevisiae. This finding is supported by previous studies, where it was shown that glycogen has a central role in fruiting body formation (Brunt and Moore, 1989). The 
accumulation of glycogen in the nodulus was also demonstrated by using histochemical methods (Matthews and Niederpruem, 1973; van der Valk and Marchant, 1978).

\subsection{Detecting tissue enhanced genes can help to understand the evolution of morphological complexity}

To detect genes that are characteristic to a particular tissue type, we introduced a formula (Sonawane et al., 2017) in the DESeq2 statistical framework. We detected genes in a tissue that showed significantly higher expression than the average expression of all tissues of the given stage using deviation contrast in a DE analysis. The genes detected with this method were termed tissue enhanced genes.

In paragraph 3.1.7., we showed that the presence of the cap, the universal veil, the partial veil and the well-developed hymenophore increased the diversification rate of species. Therefore, here we focused on tissue types related to these morphologies. We detected 2,053, 1,053, 1,260 and 941 enhanced genes in the universal veil, lamellae, cap and the partial veil of either stage, respectively. We also found that most of the genes were enhanced only in one tissue type (Figure 37.). In the cap tissue, the turnover of tissue enhanced genes was higher through the development than in the universal veil, meaning that the transcriptome of cap tissue is more actively changing. By evaluating manually curated functional annotations, we showed that the mitosis and cell-wall remodeling genes outnumbered other functional groups in all the four examined tissues (Figure 39.). This is in concordance with the fact that primordia go through rapid mass increase during the development (Moore et al., 1979), which is driven more by cell proliferation than the inflation of cells in the pre-meiotic stages (Hammad et al., 1993). It is also a well-known phenomenon that the genes that potentially modify cell wall materials are developmentally regulated through fruiting body development (Krizsán et al., 2019). In our study, we could compare different tissue types and found that different tissues shared mitosis related tissue enhanced genes to a greater extent than cell wall remodeling related tissue enhanced genes. This suggests that cell wall remodeling genes are more specific to a particular tissue than mitosis related genes. 


\subsubsection{Tissues close to the environment express defense related and surface coating genes}

We hypothesized that one of the main functions of the universal and partial veil as well as cap tissues is to give protection for the developing primordium. Protection can be achieved passively (a resistant and tenacious surface) or actively (producing molecules being toxic for microbes and fungivorous insects and nematodes). In the following, we refer to the passive protection as 'surface coating' because fungi usually produce autoassembling hydrophobic or mucigenic materials secreted to the surface of the cell. In contrast, we termed every active protection mode as 'defense'.

Based on the manually curated functional annotation lists, we found that defenserelated genes represented in the universal veil and cap tissues by a higher portion than in partial veil or lamellae. Among defense related genes, many showed a pattern where high expression was exhibited in tissues close to the environment regardless of the stage or tissue (Figure 42.). For example, a ricin-b-like lectin showed high expression in the primary hyphal knot, in all universal veil tissues, cap tissues and the upper cap tissue. Lectins constitute a diverse group with carbohydrate-binding activity and can have a role in numerous cell functions such as cell adhesion, cell proliferation and cell death (Perillo et al., 1998). Lectins have been found in higher fungi as well (Wang et al., 1998), many of them with nematotoxic and insecticide activity (Künzler, 2018). One group of nematotoxic lectins contains a ricin B domain (Pohleven et al., 2009) which was showed to have toxicity against mosquitoes (Aedes aegypti), amoebas (Acanthamoeba castellanii) and nematodes (Aphelenchus avenae, Bursaphelenchus okinawaensis) (Bleuler-Martínez et al., 2011; Tayyrov et al., 2018). In a comparative work (Krizsán et al., 2019), Ricin B like lectins were the only lectin group that was developmentally regulated in all six mushroom-forming species examined, which together with all results implies an important role of ricin B lectins in morphogenesis and defense.

It was shown that galectins, members of another lectin group, could also be toxic to nematodes, mosquitoes, fruit fly and Colorado potato beetle (Pohleven et al., 2011; Plaza et al., 2014; Tayyrov et al., 2018). The galectin 1 (cgl1) and galectin $2(\operatorname{cg} 12)$ proteins showed intense labeling in the universal veil and the partial veil (also called to lipsanoblema sensu Clémencon 2012) of the primordium of C. cinerea using an immunohistochemical method (Boulianne et al., 2000). In our study, both the cgll and cgl2 showed high expression in tissues close to the environment at a given developmental 
stage, similar to what we found in the case of Ricin B like lectins (Figure 42.). In addition, high expression of these genes was detected in the partial veil, which was also demonstrated using an immunohistochemical method (Boulianne et al., 2000).

We found 13 genes with hydrophobin domain and observed expression patterns similar to that of ricin B like lectins or galectins (Figure 42.). Hydrophobins are selfassembled hydrophobic proteins, belonging to small secreted proteins. They are characterized by eight conserved cysteine residues and are unique to the fungal kingdom (Linder et al., 2005; Linder, 2009). Hydrophobins could be specific for lifestyle (Sammer et al., 2016) and be developmentally regulated in mushroom-forming fungi (Krizsán et al., 2019). It was found that two hydrophobins were differentially expressed in the cap and the peel tissue of Agaricus bisporus (De Groot et al., 1999). It was also shown that a hydrophobin was needed for Cryphonectira parasitica to erupt under the bark of the tree (Kazmierczak et al., 2005). The role of hydrophobins in virulence of pathogenic fungi was also indicated in multiple fungal groups (Bayry et al., 2012).

Similar to hydrophobins, cerato-platanin proteins (CPPs) are self-assembled hydrophobic cysteine-rich molecules (Luti et al., 2020). They were mainly observed in plant-fungal interactions, but their role in morphogenesis has already been shown (Baccelli, 2014). The developmental regulation of CPPs was also discovered among mushroom-forming fungi (Almási et al., 2019; Krizsán et al., 2019). Here, we observed that three and one cerato-platanin coding genes were enhanced in the universal veil and the cap, respectively (Figure 42.). Furthermore, we showed that these genes were upregulated in hyphal knot stages, indicating that self-assembled hydrophobic proteins could be present through the whole developmental process of a particular tissue. One CPP (Copci_AmutBmut1_544218) exhibited similar expression patterns to that we observed in the case of hydrophobins or defense related genes (Figure 42.).

High expression levels in tissues close to the environment were detected for four wax synthase coding genes as well (Figure 43.). Wax synthases are enzymes that synthesize wax esters, a typical constituent of the cuticula of plants or the sebum of the animals (Cheng and Russell, 2004; Li et al., 2008; Schmid, 2016). Given the widespread function of wax esters in nature, it is worth considering that $C$. cinerea produces a cuticula-like layer on the surface of the fruiting body. This idea is further supported by a 
mucilaginous substance, which has been observed between hyphae of the $C$. cinerea primordia using transmission electron microscopy (van der Valk and Marchant, 1978).

It is important to note that the above-mentioned proteins could have other functions than defense or surface coating. Lectins were described as cell adhesive molecules and they could also play a role in signaling as it is described in animals (del Fresno et al., 2018) or plants (Van Holle and Van Damme, 2018) and hypothesized in fungi (Walser et al., 2005). Hydrophobins could have a wide range of functions as well. They play a role in symbiotic and pathogenic relationships, thus in interspecies interactions (Ball et al., 2019). Hydrophobins were detected on the surface of air channels in the fruiting bodies of Schizophyllum commune and Agaricus bisoprus and found to have a function in the adherence of hyphae to each other (Wessels, 1996; Lugones et al., 1999). Overall, keeping in mind that proteins could have multiple functions, our results suggest that putative defense and surface coating related genes are concentrated in tissues close to the environment, which may indicate sophisticated protection against both biotic and abiotic impacts.

\subsubsection{Both genetic predisposition and derived genes could drive the evolution of mushroom tissues}

To put the tissue enhanced genes into an evolutionary context, we wanted to examine the divergence time of each $C$. cinerea gene. We performed a phylostratigraphic analysis and parallelly, we reconstructed the ancient character states of cap, universal veil, partial veil, increased hymenophore and enclosed development traits. We found that most of the tissue enhanced genes dated to the MRCA of the Dikarya, or that of early divergent Fungi and eukaryotic ancestors preceding fungal ancestors with the fruiting body. This pattern could imply that the evolution of complex multicellularity and related structures was driven by exaptation through evolution (Gould and Vrba, 1982). Exaptation could explain the convergent evolution of a trait through coopting or predisposing features for functions that were not built for their current role. Genetic predisposition through the evolution of multicellular organisms is a well-known phenomenon (Nagy et al., 2014; Niklas et al., 2020) and morphological evolution is frequently driven by altering the expression of functionally conserved genes (Carroll, 2008). It was recently shown that simple multicellularity probably evolved in fungi via the co-option of ancient eukaryotic genes (Kiss et al., 2019). It was also shown that a highly similar genetic toolkit was used 
to develop rhizomorph and stipe of Armillaria ostoyae (Sipos et al., 2017). Based on fossil data, rhizomorph like structures could have existed before the evolution of fruiting bodies (Auxier et al., 2016; Smith, 2016), therefore it is an interesting hypothesis that an ancient genetic toolkit could have existed for developing various complex structures. By examining the transcriptome of nine complex multicellular species from Agaricomycotina and Pezizomycotina, Merényi et al. (Merényi et al., 2020) found pieces of evidence for molecular convergence (i.e., similar genetic changes in the genomes on phylogenetically unrelated lineages) (Castoe et al., 2010). They showed that the majority of fruiting body related genes underwent expansion independently in the Agaricomycotina and Pezizomycotina subphyla. Based on our ASR analysis and previous studies (Reijnders, 1977; Hibbett, 2007), the cap, the universal and the partial veil convergently evolved in multiple mushroom groups. Therefore, the prevalence of ancient genes among tissue enhanced genes may imply deep homology (Shubin et al., 2009). In our study, we did not infer gene duplication and loss events, yet the hypothesis of molecular convergence below the subphylum level may be worth testing in the future.

Our results fit well in previous findings (e.g., Krizsán et al., 2019; Merényi et al., 2020), however, we detected an enrichment of a relatively young gene set among universal veil enhanced genes. These 40 genes were dated to the stem node of the Marasmioid clade sensu Matheny (Matheny et al., 2006). Interestingly the phenotypic inventions such as the cap, universal veil, enclosed development and well-developed hymenophore were reconstructed to or next to this node by maximum parsimony-based ASR analysis. Moreover, based on the molecular clock analyses, the stem node of the Marasmioid clade dated to the Jurassic period, where we detected an abrupt increase in the diversification of species. Among the 40 genes, we detected three transcription factors (TFs) belonging to the C2H2-type, GATA-type and SIX-type families. Furthermore, two proteins with F-box domain were also found. Krizsán et al. (Krizsán et al., 2019) observed the expansion of developmentally regulated F-box proteins in the class Agaricomycetes. They also found a high number of developmentally regulated transcription factors, including $\mathrm{C} 2 \mathrm{H} 2$-type TFs. Previous studies on the multicellularity of fungi suggested that species were genetically predisposed for the evolution of higher morphological complexity (Nagy et al., 2014, 2020; Kiss et al., 2019; Krizsán et al., 2019; Merényi et al., 2020). However, here we detected a relatively young gene set which enhanced in the universal veil and enriched in an agaricoid ancestor (stem node of the Marasmioid clade) 
dated to the Jurassic period, showing the intricate evolutionary history of complex multicellularity in fungi.

\subsection{Conclusions}

With the emergence of modern bioinformatics methods such as phylogenomics (Eisen and Fraser, 2003) or phylogenetic comparative methods (Pagel, 1999), new opportunities were opened up to test the nature of the evolution of species. One of the most significant innovations during the evolution was forming complex multicellular structures having an immense impact on the present life (Szathmáry and Maynard Smith, 1995; Knoll, 2011). Our knowledge on the evolution of complex multicellularity in fungi is scarce, even though they have an important role in the ecosystem functioning. The Agaricomycetes, also known as mushroom-forming fungi, is the largest clade in fungi, which consists of more than $>25,000$ complex multicellular species. That is why we examined the evolution of these species using modern bioinformatics and transcriptomic methods. We found that the mushroom-forming fungi went through an expansion during the Jurassic period, which coincided with the evolution of complex fruiting body types. Complex fruiting bodies such as coralloid, pileate-stipitate or gasteroid mushrooms start their FB development with the formation of hyphal knots. Hyphal knots have a fundamental role in mushroom development (Reijnders and Moore, 1985; Clémencon, 2012; Kües and Navarro-González, 2015), consequently, we put under scrutiny these structures using a low-input tissue-specific RNA-seq. We found that cell division and cell wall remodeling genes have a pivotal role in the transition from vegetative mycelium into the complex multicellular hyphal knot. In later developmental stages, mushrooms develop tissues (cap, universal and partial veils), which we found had a positive effect on the diversification of species during the evolution of Agaricomycetes. The results of the phylogenetic comparative analyses encouraged us to hypothesize that these tissues could have a function in the passive and active protection of the developing FB. Accordingly, we detected more than a thousand of cap, universal veil and partial veil enhanced genes among them with numerous defense and surface coating related genes. This result gives an example of creating a functional hypothesis on genes by combining PCM and transcriptomic analyses. Furthermore, we also tried to unravel the connection between phenotypic and genetic evolution by examining the level of conservation of tissue enhanced genes using phylogenomic methods. We found that most of the tissue enhanced 
genes evolved in dikaryotic or ancient fungal and eukaryotic ancestors. Our PCM analyses showed that the cap, the universal and partial veil are convergent structures thus the evolution of these tissues could have mainly been driven by exaptation. An exception was an enriched set of genes among universal veil enhanced genes that evolved at the stem node of the Marasmioid clade sensu Matheny. This implies that phenotypic evolution of $C$. cinerea could be driven by mainly ancient and partially young genes as well.

Overall, by combining phylogenomic, transcriptomic and comparative phylogenetic methods, we disentangled a piece of the evolution of mushroom-forming fungi and showed that the evolution complex multicellularity among fungi could have been the result of intricate genetic and macro-evolutionary events. 


\section{Summary}

Several major transitions have happened through the evolution of life, such as the evolution of eukaryotes, plastids, or multicellularity. Multicellular organisms exist in all biogeographic realms and are essential components of most present ecosystems. Simple multicellular organisms form aggregates or colonies of cells and have arisen in both prokaryotic and eukaryotic lineages at least 25 times. In contrast, complex multicellularity, where three-dimensional structures are developed and only some of their cells are in direct contact with the environment, evolved exclusively in five eukaryotic lineages from which three dominate the present terrestrial ecosystems: animals, embryophytic land plants and fungi. Despite the prevalence of complex multicellularity on Earth, the driving force and the genetic background of the evolution of complex multicellular organisms are incompletely known. Both biotic and abiotic factors could drive the evolution of complex multicellularity such as changes in feeding mode, increase in atmospheric oxygen, or whole-genome duplications. Pieces of evidence also mount that the evolution of multicellular organisms was driven by exaptation, a mechanism where a pre-existing genetic toolkit of ancestors can give new adaptive features for descendants.

The fungal kingdom is one of the five main groups where complex multicellularity evolved, but complex multicellularity could have convergently appeared at least eight times within fungi. The convergent evolution of complex multicellularity in the fungal kingdom implies a unique evolutionary solution for developing complex structures. Fungal complex multicellular structures are bound to a particular life period (fruiting bodies) or circumstances (mycorrhizae, rhizomorphs), and these are only a part of the whole organism. In contrast to this, the whole individual is the complex multicellular entity in other complex multicellular organisms. Possibly, the most common fungal complex multicellular structures are the sexual fruiting bodies, whose primary purpose is to produce meiotic spores in a protective environment and facilitate spore dispersal. Agaricomycetes, also called mushroom-forming fungi, contain more than 20,000 species, which produce fruiting bodies. Therefore, examining this class of fungi with phylogenetic comparative methods (PCMs) would widen our knowledge on the macro-evolution of complex morphologies in fungi. Previous studies have examined small datasets or separate Agaricomycetes taxa using PCMs. So far, it is revealed that the evolution of 
mushrooms could have started as a resupinate ancestor (crust-like fruiting body) and through a coralloid/clavarioid type, pileate-stipitate mushrooms (fruiting body with cap and stipe) convergently evolved. It was also showed that pileate-stipitate fruiting bodies could increase the diversification rate of lineages, indicating that this morphology bears advantageous traits for species. However, the global evolutionary history of the Agaricomycetes and some of the key traits of pileate-stipitate mushrooms (presence of cap, protecting veils, structured hymenophore) have not been examined using sufficiently large and robust data.

Therefore, we gathered specimens of Agaricomycetes from every geographical region except Antarctica and assembled a dataset containing 5,284 species and three genomic loci (nrLSU, rpb2, tef1- $\alpha$ ). Using this dataset, we performed maximum likelihood (ML) inference, and we constrained the topology of the backbone based on a phylogenomic tree consist of 105 species. Overall, $245 \mathrm{ML}$ trees were inferred, covering the topological variety of Agaricomycetes phylogeny based on Robinson-Foulds pairwise distances. We also performed molecular clock calibration on ten trees selected by stratified random sampling to represent the topology diversity of the 245 trees. The molecular clock calibration was done by a two-step time calibration strategy. This procedure was based on using a robust and precise method on the ten percent of the species to infer parameters. Then, the time calibration of trees with all species was performed using a less precise but a fast algorithm to which the parameters inferred in the first step was inputted. Most of the order-level clades were dated to the Jurassic period (200-145 Ma ago), including the two subclasses Agaricomycetidae and Phallomycetidae. These ages are older than the time estimates of previous studies, therefore we tried to disentangle the underlying causes by reconstructing time-calibrated phylogenomic trees with various settings to perform a sensitivity analysis. Overall, we found that the precise placement of fossil calibration point on the phylogeny has the most significant effect on age estimates, and the number of fossil calibration points, the type of the tree (phylogenetic vs. phylogenomic) and the software choice have a smaller effect on the result of the time calibration. Therefore, we think our analyses provide a robust and statistically well-supported divergence time estimates for lineages of mushroom-forming fungi.

Using the ten time-calibrated trees (chronograms), we performed a trait independent diversification analysis using the BAMM model. We inferred branch- 
specific speciation and extinction rates and summarized the net diversification rates (speciation minus extinction rates) over time. We found that the diversification rate abruptly increased in the Jurassic period (200-145 Ma ago). A small increase in the net extinction rate was also detected at the end of the Jurassic period. Thus, we further scrutinized whether mass extinction could have occurred through the evolution of Agaricomycetes using the CoMET model. This analysis also supported the presence of an extinction event at the end of the Jurassic period. Interestingly, no sign for a mass extinction at the Cretaceous-Paleogene (K-Pg) boundary was detected. Therefore, mushroom-forming fungi probably did not suffer severe extinction at the K-Pg boundary as most of the main groups did.

We also inferred significant congruent core shifts in the speciation rate. Overall, 85 congruent core shifts were detected, from which 57 occurred at the MRCA of clades consisting of species with exclusively pileate morphologies. We also performed ancestral character state reconstruction of the main fruiting body types, and we found that the complex morphologies (pileate, gasteroid, coralloid) evolved in the Jurassic and the proportion of lineages with pileate morphology among Agaricomycetes lineages have been increased. This, and that many significant core shifts related to clades with pileate morphologies imply that complex fruiting bodies could bear morphological traits which evolutionary favorable for Agaricomycetes species. That is why we examined five character states using trait dependent diversification models. We found that the presence of cap, the enclosed development, the presence of universal and/or partial veil, and the increased hymenophore surface area significantly increased the diversification rate of species. Our analyses showed that complex fruiting bodies with morphological traits such as the cap or veils could evolutionarily favorable for species, therefore we asked what gene lies behind the development of mushrooms and morphological traits like cap or veils.

To answer this question, we aimed to thoroughly examine tissue-specific transcriptome through the early development of Coprinopsis cinerea model mushroom. First, we examined the early developmental and tissue formation of $C$. cinerea by inspecting more than 200 cryo-sections. Then, we worked out an RNA sequencing coupled with laser capture microdissection (LCM) workflow by adapting and modifying LCM and RNA-seq methods to $C$. cinerea. Nine tissue types and seven developmental stages were selected to be involved in our RNA-seq study: vegetative mycelium, primary 
hyphal knot, secondary hyphal knot, primordium initial, stage 0 primordium, stage 1 primordium and stage 2 primordium. In this study, we produced 84 cDNA libraries representing all the tissue types with 2-4 biological replicates. First, we performed differential expression analyses between vegetative mycelium and hyphal knot stages to disentangle the transcriptome changes through the first steps of the mushroom development. We detected 1,293-2,590 upregulated genes in the tissues of primary and secondary hyphal knots. We found that cell division and cell wall remodeling genes are enriched and could have a pivotal role in the transition from vegetative mycelium into the complex multicellular hyphal knot. To detect tissue enhanced genes in later stages, we adapted a formula into the DESeq2 statistical framework and performed generalized linear model analyses with deviation contrast. In this analysis, we detected those genes that had significantly higher expression in a tissue than the average expression of all tissues in a given developmental stage. We found more than a thousand of cap, universal veil and partial veil enhanced genes among them with numerous defense and surface coating related genes. Furthermore, we also tried to unravel the connection between phenotypic and genetic evolution by examining the level of conservation of tissue enhanced genes using phylogenomic methods. We found that most of the tissue enhanced genes evolved in dikaryotic or ancient fungal and eukaryotic ancestors. This means that tissue enhanced genes predated the evolution of complex structures, and the evolution of tissues could have mainly been driven by exaptation. An exception was an enriched set of genes among universal veil enhanced genes that evolved at the stem node of the Marasmioid clade, which implies that the phenotypic evolution of $C$. cinerea could be driven by mainly ancient and partially young genes as well.

In our study, we combined phylogenomic, transcriptomic and comparative phylogenetic methods to disentangle the evolution of Agaricomycetes. Using cutting edge bioinformatics and transcriptomic methods, we found a connection between the evolution of genes and phenotypic traits and showed that the evolution complex multicellularity in fungi could have been the result of intricate genetic and macro-evolutionary events. 


\section{Összefoglalás}

A soksejtü élőlények az összes biogeográfiai régióban elterjedtek és jelentős szerepet töltenek be a jelen ökoszisztémák müködésében. Az egyszerü soksejtü élölények sejt aggregációkat vagy kolóniákat hoznak létre és több mint 25 alkalommal alakultak ki mind prokarióta és eukarióta ősökben. Ezzel szemben, a komplex soksejtü élőlények öt alkalommal alakult ki kizárólag eukarióta csoportokban, amelyek közül három a szárazföldi ökoszisztémák meghatározó résztvevői: állatok, növények és gombák. Ezen élőlényekre jellemző a bonyolult három-dimenziós testfelépítés létrejötte, valamint, hogy nem minden sejt áll közvetlen kapcsolatban a környezettel. A komplex soksejtüség jelentősége és széles elterjedése ellenére ismereteink limitáltak a kialakulásával és genetikai hátterével kapcsolatban. A soksejtüség kialakulásában valószínüleg egyaránt játszhattak szerepet biotikus és abiotikus tényezők, úgy, mint a táplálkozás módban történő változások, a légköri oxigén koncentráció megváltozása vagy teljes genom duplikációs események. Továbbá, egyre több jel mutat arra, hogy a komplex soksejtüség egyik fő evolúciós mozgató rugója az úgynevezett exaptáció lehet. Az exaptáció azt a jelenséget írja le amikor az ősökben már létező genetikai eszköztár egy új adaptív tulajdonságot nyújt a leszármazottakban.

A gombák országa az öt komplex soksejtü eukarióta csoport egyike. A komplex soksejtü képletek evolúciója gombákon belül sajátos utat követhetett a többi élőlénycsoporthoz képest ugyanis a gombákon belül legalább nyolc alkalommal egymástól függetlenül evolválódtak komplex soksejtü leszármazási vonalak. A gombák komplex soksejtủ struktúrái nagyrészt egy adott életszakaszhoz (például termőtest) vagy körülményhez (ektomikorrhiza, rhizomorfa) kötött. Ezzel szemben, más komplex soksejtü taxonban az egész egyed maga a soksejtü struktúra. Az egyik leggyakoribb komplex soksejtü képlet a gombákon belül a termőtest, aminek az elsődleges funkciója a meiospórák termelése egy relatíve védett környezetben, valamint a spóra terjesztés elősegítése. Az egyik legnagyobb gombacsoport, ahol termőtest képző gombák találhatók a Agaricomycetes osztály vagy másnéven termőtest képző gombák osztálya. Az Agaricomycetes osztály több mint 20000 fajt tartalmaz, amelyek nagyrésze komplex soksejtü képleteket képeznek. Tehát a komplex soksejtű struktúrák evolúciójáról számos új ismeretre tehetünk szert, ha a termőtest képző gombák osztályát megvizsgáljuk filogenetikai komparatív módszerek (PCM) használatával. Számosz kutató végzet már 
elemzéseket kisebb adatsorokon vagy bizonyos Agaricomycetes kládokon PCM alkalmazásával. Ezekből már valószínüsíthető, hogy a kalapos-tönkös (pileate-stipitate) termőtestek reszupinátus (kéreg szerü aljzaton elterülö) ősökböl származnak koralloid átmeneti formán keresztül. Azt is kimutatták, hogy a kalapos-tönkös termőtest típus megnövelte a fajok diverzifikációs rátáját (fajképződési ráta és a kihalási ráta különbsége), ami arra utalhat, hogy a komplex termőtest típusok rendelkeznek bizonyos morfológiai karakterekkel, amelyek előnyösek lehetnek a fajok számára. Viszont a globális evolúciós mechanizmusok, amelyek az Agaricomycetes osztályt kialakították, valamint bizonyos kulcs morfológiai innovációk (kalap jelenléte, zártan fejlődő termőtest, termőréteg felület növelés) hatása a fajok diverzifikációs rátájára nem vagy kevésbé ismert.

Ezért kutatócsoportunk az Antarktisz kivételével minden földrajzi régióból gyüjtött Agaricomycetes fajokat, és összeállított egy 5284 fajt és három genomi lókuszt (nrLSU, rpb2, tef1- $\alpha$ ) tartalmazó adatsort. Ezt az adatsort felhasználva egy maximum likelihood (ML) elemzést készítettünk, ami során a fö topológiát fixáltuk egy 105 fajból álló filogenomikai fa alapján. Összességében $245 \mathrm{ML}$ fa készült el, ami lefedi az Agaricomycetes filogenetikai topológiai variabilitását Robinson-Foulds távolságok összegzése alapján. Tíz fa molekuláris órájának kalibrálását is elvégeztük, amelyeket rétegzett véletlenszerü mintavétellel választottunk ki a 245 ML fa közül. A molekuláris óra kalibrálást egy kétlépcsős Bayes-féle elemzéssel végeztük el. Elsőként egy robusztus és pontos módszert alkalmazva a fajok tíz százalékát használva becsültük meg a molekuláris óra kalibrációhoz szükséges paramétereket. Ezután a fák időbeli kalibrálását minden faj felhasználásával egy kevésbé precíz, de gyors algoritmus segítségével hajtottuk végre, ami során az első lépés során becsült paramétereket alkalmaztuk. Az így kapott fák (kronogrammok) elemzéséből kiderült, hogy a rendszintü kládok többsége a Jura időszakra (200-145 millió évvel ezelőtt) volt tehető, ideértve a két alosztályt: Agaricomycetidae és Phallomycetidae. Ezek a becsült korok idősebbek, mint amit a korábbi vizsgálatok kimutattak. Ezért megpróbáltuk kideríteni, hogy mi állhat e különbség mögött, amit egy 105 faj genomi adatát felölelő kronogram elemzésével, és különböző paraméterek vizsgálatával tettünk meg. Összességében azt tapasztaltuk, hogy a fosszília kalibrációs pontok lokalizációja van a legnagyobb hatással a korbecslésre, és a fosszília kalibrációs pontok száma, a fa típusa (filogenetikai vagy filogenomikai) és a szoftver választása kisebb hatással van a molekuláris óra kalibráció eredményére. Ezért 
úgy gondoljuk, hogy elemzéseink robusztus és statisztikailag jól alátámasztott divergencia-időbecslést nyújtanak a termőtest képző gombák osztályára nézve.

Tíz kronogram felhasználásával karakter független diverzifikációs ráta elemzést végeztünk a BAMM modellt alkalmazva. Megbecsültük az ág-specifikus fajképződési és kihalási rátákat majd összegeztük a nettó diverzifikációs rátákat (fajképződés és kihalási ráták különbsége) az evolúciós idő mentén. Megállapítottuk, hogy a diverzifikációs ráta hirtelen növekedésnek indult a Jura időszakban. A nettó kihalási ráta kismértékü növekedését is detektáltuk a Jura kor végén, ezért a CoMET modell segítségével tovább vizsgáltuk, hogy történhetett-e tömeges kihalás az Agaricomycetes evolúciója során. A CoMET modell segítségével végzett elemzések támogatták egy kihalási esemény jelenlétét a Jura időszak végén. Érdekes módon a kréta-paleogén (K-Pg) határán nem detektálunk tömeges kihalás jeleit, ahol viszont más élőlénycsoportokban a fajok $75 \%$-a kihalt.

Fajképződési rátában bekövetkező pontszerü szignifikáns változásokat is detektáltunk a BAMM modell segítségével. Összességében 85 kongruens fajképződési rátaváltozást azonosítottunk, amiből, 57 olyan kládokban fordult elő, amelyeket kizárólag kalapos termőtestű (pileate típusú) fajok alkotnak. Ösi karakterállapot rekonstrukciót is végeztünk a fö termőtesttípusokra nézve és azt tapasztaltuk, hogy a bonyolult morfológiák (pileate, gaszteroid, koralloid) a Jura korszakban fejlődtek ki, és később az Agaricomycetes leszármazási vonalak között megnőtt a pileate morfológiával rendelkező csoportok aránya. Az ősi karakterállapot rekonstrukció elemzés eredménye, és hogy a fajképződési ráta változás nagyrészt pileate morfológiájú kládokhoz köthetők azt mutatják, hogy a komplex termőtestek olyan morfológiai tulajdonságokat hordozhatnak, amelyek evolúciós szempontból kedvezőek lehetnek az Agaricomycetes fajok számára. Ezért megvizsgáltunk öt morfológiai karaktert karakterfüggő diverzifikációs modellek (BiSSE vagy MuSSE) felhasználásával. Megállapítottuk, hogy a kalap jelenléte, a zártan történő termőtest fejlődés, az általános és/vagy a részleges burok, valamint a spóraképző felület növelése mind pozitívan befolyásolta a fajok diverzifikációs rátáját. Elemzéseink azt mutatták, hogy az olyan morfológiai tulajdonságokkal rendelkező komplex termőtestek, mint a kalap vagy különböző burkok, evolúciós szempontból kedvezőek lehetnek a fajok számára. Ezért továbbiakban arra voltunk kíváncsiak, hogy milyen gének játszanak szerepet a termőtest képzésében, valamint olyan morfológiai képletek képzésében, mint a kalap, az általános és részleges burok. 
Hogy ezeket a kérdéseket megválaszoljuk egy szövetspecifikus transzktiptomikai vizsgálatot végeztünk a Coprinopsis cinerea modell gomba korai termőtest fejlődése során. Elöször több mint 200 krio-hisztológiai vizsgálat elvégzésével tanulmányoztuk a C. cinerea korai fejlődését és szövet képzését. Ezután kidolgoztunk egy RNSszekvenálást, amit lézer mikrodisszekciós technikával (LCM) kombináltunk és számos eljárás módosításával adaptáltuk $C$. cinerea modell gombára. Kilenc szövettípust és hét fejlődési stádiumot választottunk ki a $C$. cinerea korai fejlődésnek transzkriptomikai vizsgálatára: vegetatív micélium, elsődleges hifa csomó, másodlagos hifa csomó, primordium iniciális, primordium 0 stádium, primordium 1 stádium és primordium 2 stádium. Jelen tanulmányban 84 cDNS könyvtárat állítottunk elö, amelyek az összes szövettípust lefedve 2-4 biológiai ismétlést tettek lehetővé. Először differenciális génexpressziós elemzéseket végeztünk a vegetatív micélium és a hifa csomó fejlődési állapotok között, hogy a termőtest fejlődés első lépéseiben szerepet játszó géneket megvizsgáljuk. Az elsődleges és a másodlagos hifa csomók szöveteiben 1293 - 2590 felülregulált gént detektáltunk. Megállapítottuk, hogy a sejtosztódásban és a sejtfal szerkezet módosításában szerepet játszó gének feldúsultak ezekben a szövetekben, és kulcsfontosságúak lehetnek a vegetatív micéliumból a komplex többsejtü hifa csomóba történő átmenetben. Összességében 161 felülregulált gént találtunk az első két fejlődési szakaszban, amelyek összefüggésbe hozhatók a sejtosztódással és a hifa növekedési mintázat szabályozásával, beleértve a ciklineket, a STRIPAK fehérjéket és a szeptineket. Megállapítottuk, hogy ezeknek a géneknek a száma az első fejlődési állapotok során megnőtt. Továbbá detektáltunk 112, a sejtfal biogenezisével és átalakításával kapcsolatos, felül szabályozott gént is, köztük 75 feltételezett glikozid-hidroláz (GH) aktivitású gént.

A továbbiakban szövet-specifikus géneket szerettünk volna azonosítani, hogy a makro-evolóciós elemzéseink során vizsgált jellegekhez kapcsolódó szövetek genetikai hátterét tanulmányozhassuk. Ehhez a DESeq2 program csomag statisztikai környezetét használva általánosított lineáris modellt használtunk, deviancia kontraszt alkalmazásával. Az elemzés során olyan géneket azonosítottunk egy adott szövetben, amely szignifikánsan magasabb génexpressziót mutatott, mint az adott stádium összes szövetének átlagos expressziója. Összesen 2053, 1053, 1260 és 941 felülregulált gént detektáltunk az általános, részleges burok, a kalap, illetve lemezek szöveteiben. Feltételeztük, hogy az általános, a részleges burkok illetve a kalap egyik fő funkciója a fejlődő priordium védelme. A manuálisan összegzett funkcionális annotációs listák 
alapján azt találtuk, hogy a védelemmel kapcsolatos gének az általános burok és kalap szöveteiben nagyobb arányban találhatóak, mint a részleges burokban vagy a lemezben. Továbbá számos védelemben szerepet játszó gén olyan mintázatot mutatott, amely a környezethez közeli szövetekben magas expresszióval rendelkezett. Ezenkívül megpróbáltuk feltárni a fenotípusos és genetikai evolúció kapcsolatát a szövet-specifikus gének konzerváltság szintjének filosztratigráfiai elemzésével. Megállapítottuk, hogy a szövet-specifikus gének nagy része Dikarya vagy alacsonyrendü gomba- és eukarióta ősökben fejlödött ki. Ez azt jelenti, hogy a szövet-specifikus gének megjelenése megelőzte a komplex morfológiai struktúrák és a szövetek evolúcióját. Ez alól kivételt képzett egy általános burok specifikus géncsoport, amely a Marasmioid-klád ösében alakulhatott ki. Eredményeink összességében azt sugallják, hogy a C. cinerea fenotípusos evolúcióját főként ősi és részben fiatal gének alakították.

Vizsgálatunk során filogenomikai, transzkriptomikai és filogenetikai összehasonlító módszereket kombináltunk az Agaricomycetes osztály evolúciójának vizsgálatához. A legmodernebb bioinformatikai és transzkriptomikai módszerek segítségével összefüggést találtunk a gének evolúciója és a fenotípusos tulajdonságok között, és kimutattuk, hogy a komplex soksejtüség bonyolult genetikai és makroevolúciós események eredménye következtében jöhetett létre a gombák országában. 


\section{Acknowledgment}

I would like to express my deepest appreciation to my supervisor, dr. László G. Nagy for his help and mentorship throughout my doctoral work. I am also thankful for the opportunities he provided me to participate in such great and inspiring projects. I genuinely enjoyed being part of the Fungal Genomics and Evolution Lab and want to thank all the lab members for their help and the cheery company they provided me.

I would like to thank the co-authors contributing to our study with their tremendous work. dr. Krisztina Krizsán produced phylogenomic tree of Agaricomycetes, which was used as a backbone in the tree inference of the 5,284 species dataset. She also helped me to initiate my first experiences in the lab. Viktória Bense handled and grew $C$. cinerea strain in the lab and helped in character state coding of mushrooms. Csenge Földi also helped in character state coding, and she performed BayesTraits analyses of the increased hymenophore area trait. dr. Marisol Sánchez-García (Swedish University of Agricultural Sciences) performed BiSSE analysis using the cap trait data. dr. Zsolt Merényi inferred the genomic tree of 441 species, which was used in the phylostratigraphic analysis. Dr. Balázs Bálint and dr. Zsolt Merényi helped me in RNA-seq read alignment procedures. We also want to thank István Nagy for writing a custom java program to screen Agaricomycetes species at the Species Fungorum database. We want to say thank dr. Gergely J. Szöllősi (Eötvös Loránd University), who put much energy in the molecular clock calibration of the 5,284-species dataset, and helped us to work out the two-step Bayesian molecular clock calibration procedure. I also want to thank dr. Michael Love (University of North Carolina-Chapel Hill, USA) for suggesting using alternative GLM contrast methods within the DESeq2 framework. We are also grateful for the suggestions and comments of dr. David S. Hibbett (Clark University, USA). We would like to thank Prof. dr. Ursula Kües (University of Göttingen, Germany) for providing us the C. cinerea A43mutB43mut strain. We would like to express our gratitude to dr. Péter Horváth for providing us the Zeiss PALM MicroBeam microscope, and to Árpád Bálind for demonstrating the usage of this instrument. We would like to thank dr. Anikó KellerPintér for temporally providing us access for the cryo-microtome at the Department of Biochemistry, Faculty of Medicine, University of Szeged. We are grateful for all the collectors and researchers who gathered and sent specimens from worldwide. We thank many colleagues for participating in the sequencing of specimens. Special thanks go to 
dr. Sándor Kocsubé (University of Szeged) and dr. Bálint Dima (Eötvös Loránd University) and the members of the Joint Genome Institute, USA. I also want to thank the conscientious work of dr. Sándor Kocsubé (University of Szeged) and dr. Károly Kovács (Biological Research Centre, Hungary) through the home defense procedure.

I also want to say thanks to the following societies and scientific communities for their efforts of organizing events which helped me in improving my communication skills and provided an excellent environment to meet relevant researcher from my field: Hungarian Microbiological Society, Hungarian Mycological Society, Mycological Society of America, Hungarian Research Student Association, Mushroom Society of Zemplén. I would like to thank the following grants and scholarships for their financial support: Nemzeti Tehetség Program (NTP-NFTÖ-19-B-0139), Új Nemzeti Kiválósági Program ösztöndíj (UNKP-18-3-IV-SZTE-52), Nemzeti Tehetség Program (NTP-NFTÖ17-B-0337), Nemzeti Tehetség Program ösztöndíja (NTP-EFÖ-P15), Straub Fiatal Kutatói Díj (Szegedi Biológiai Kutatóközpont). Our researches were also supported by the Lendület grant LP2014/12; OTKA NN106394; GINOP-2.3.2-15-2016-00001.

Last but not least, I would like to thank my family for their support, especially to my partner, Gellén Barbara, who, despite the difficulties she encountered due to my occasionally fanatic engagement in my work, have always supported me and cheered me up with her charming presence. Thanks should also go to my friends from high school and from university who organized lovely weekend programs and other events which brought pleasant moments to my life. 


\section{References}

Acharya, B.R. and A.S. Yap (2016), Cell-Cell Adhesion and the Cytoskeleton, Encyclopedia of Cell Biology 2, 704-712.

Agerer, R. (2006), Fungal relationships and structural identity of their ectomycorrhizae, Mycological Progress 5 (2), 67-107.

Agger, J.W. et al. (2014), Discovery of LPMO activity on hemicelluloses shows the importance of oxidative processes in plant cell wall degradation, Proceedings of the National Academy of Sciences of the United States of America 111 (17), 6287-6292.

Akerborg, Ö., B. Sennblad and J. Lagergren (2008), Birth-death prior on phylogeny and speed dating, BMC Evolutionary Biology 8 (1), 77.

Alexa, A. and J. Rahnenfuhrer (2020), topGO: Enrichment Analysis for Gene Ontology, $\mathrm{R}$ package version 2.38.1.

Alexa, A., J. Rahnenführer and T. Lengauer (2006), Improved scoring of functional groups from gene expression data by decorrelating GO graph structure, Bioinformatics 22 (13), 1600-1607.

Alfaro, M.E. et al. (2009), Nine exceptional radiations plus high turnover explain species diversity in jawed vertebrates, Proceedings of the National Academy of Sciences 106 (32), 13410-13414.

Almási, É. et al. (2019), Comparative genomics reveals unique wood-decay strategies and fruiting body development in the Schizophyllaceae, New Phytologist 224 (2), 902-915.

Andrews, S., F. Krueger, A. Segonds-Pichon, L. Biggins, C. Krueger and S. Wingett (2018), FastQC: a quality control tool for high throughput sequence data.

Arcila, D. and J.C. Tyler (2017), Mass extinction in tetraodontiform fishes linked to the Palaeocene-Eocene thermal maximum, Proceedings of the Royal Society B: Biological Sciences 284 (1866).

Arima, T., M. Yamamoto, A. Hirata, S. Kawano and T. Kamada (2004), The eln3 gene involved in fruiting body morphogenesis of Coprinus cinereus encodes a putative membrane protein with a general glycosyltransferase domain, Fungal Genetics and Biology 41 (8), 805-812.

Ashburner, M. et al. (2000), Gene Ontology: tool for the unification of biology, Nature Genetics 25 (1), 25-29.

Auxier, B. et al. (2016), No place among the living: phylogenetic considerations place the Palaeozoic fossil T. protuberans in Fungi but not in Dikarya. A comment on M. Smith (2016), Botanical Journal of the Linnean Society 182 (4), 723-728.

Baccelli, I. (2014), Cerato-platanin family proteins: one function for multiple biological roles?, Frontiers in plant science 5, 769.

Baker, S.C. et al. (2005), The external RNA controls consortium: A progress report, Nature Methods 2 (10), 731-734.

Ball, S.R., A.H. Kwan and M. Sunde (2019), Hydrophobin Rodlets on the Fungal Cell Wall, Current Topics in Microbiology and Immunology, Springer, Berlin, Heidelberg.

Bayry, J., V. Aimanianda, J.I. Guijarro, M. Sunde and J.P. Latgé (2012), Hydrophobinsunique fungal proteins, PLoS Pathogens 8 (5), 6-9.

Belmonte, M.F. et al. (2013), Comprehensive developmental profiles of gene activity in regions and subregions of the Arabidopsis seed, Proceedings of the National Academy of Sciences 110 (5), E435-E444. 
Benjamini, Y. and Y. Hochberg (1995), Controlling the false discovery rate: a practical and powerful approach to multiple testing, Journal of the Royal Statistical Society B 57 (1), 289-300.

Benton, M.J. and R.J. Twitchett (2003), How to kill (almost) all life: The end-Permian extinction event, Trends in Ecology and Evolution 18 (7), 358-365.

Berbee, M.L., T.Y. James and C. Strullu-Derrien (2017), Early Diverging Fungi: Diversity and Impact at the Dawn of Terrestrial Life, Annual Review of Microbiology 71 (1), 41-60.

Blackburn, D.G. (2015), Evolution of vertebrate viviparity and specializations for fetal nutrition: A quantitative and qualitative analysis, Journal of Morphology 276 (8), 961-990.

Bleuler-Martínez, S. et al. (2011), A lectin-mediated resistance of higher fungi against predators and parasites, Molecular Ecology 20 (14), 3056-3070.

Bloemendal, S. et al. (2012), A homologue of the human STRIPAK complex controls sexual development in fungi, Molecular Microbiology 84 (2), 310-323.

Bodensteiner, P., M. Binder, J.M. Moncalvo, R. Agerer and D. S. Hibbett (2004), Phylogenetic relationships of cyphelloid homobasidiomycetes, Molecular Phylogenetics and Evolution 33 (2), 501-515.

Bollback, J.P. (2006), SIMMAP: stochastic character mapping of discrete traits on phylogenies., BMC bioinformatics 7, 88 .

Bonneville, S. et al. (2020), Molecular identification of fungi microfossils in a Neoproterozoic shale rock, Science Advances 6 (4).

Botling, J. et al. (2009), Impact of Thawing on RNA Integrity and Gene Expression Analysis in Fresh Frozen Tissue, Diagnostic Molecular Pathology 18 (1), 44-52.

Boulianne, R.P., Y. Liu, B.C. Lu, U. Kües and M. Aebi (2000), Fruiting body development in Coprinus cinereus: regulated expression of two galectins secreted by a non-classical pathway, Microbiology 146 (8), 1841-1853.

Brand, A. and N.A. Gow (2009), Mechanisms of hypha orientation of fungi, Current Opinion in Microbiology 12 (4), 350-357.

Bromham, L., S. Duchêne, X. Hua, A.M. Ritchie, D.A. Duchêne and S.Y.W. Ho (2018), Bayesian molecular dating: Opening up the black box, Biological Reviews.

Brown, R.W. (1940), A bracket fungus from the late Tertiary of southwestern Idaho, Journal of the Washington Academy of Sciences 30 (10), 422-424.

Bruneau, A., B.B. Klitgaard, G. Prenner, M. Fougère-Danezan and S.C. Tucker (2014), Floral evolution in the detarieae (Leguminosae): Phylogenetic evidence for labile floral development in an early-diverging legume lineage, International Journal of Plant Sciences 175 (4), 392-417.

Brunet, T. and N. King (2017), The Origin of Animal Multicellularity and Cell Differentiation, Dev Cell 43 (2), 124-140.

Brunt, I.C. and D. Moore (1989), Intracellular glycogen stimulates fruiting in Coprinus cinereus, Mycological Research 93 (4), 543-546.

Bullard, J.H., E. Purdom, K.D. Hansen and S. Dudoit (2010), Evaluation of statistical methods for normalization and differential expression in mRNA-Seq experiments, BMC Bioinformatics 11 (1), 94.

Bushnell, B. (2020), BBMap short read aligner, and other bioinformatic tools, sourceforge.net/projects/bbmap/, retrieved 1.1.2020.

Bussolati, G., L. Annaratone, E. Medico, G. D’Armento and A. Sapino (2011), Formalin fixation at low temperature better preserves nucleic acid integrity, PLoS ONE 6 (6).

Bustillos, J. et al. (2020), Uncovering the Mechanical, Thermal, and Chemical 
Characteristics of Biodegradable Mushroom Leather with Intrinsic Antifungal and Antibacterial Properties, ACS Applied Bio Materials 3 (5), 3145-3156.

CABI (2018), Species Fungorum.

Camacho, C. et al. (2009), BLAST+: architecture and applications, BMC

Bioinformatics 10 (1), 421.

Carbon, S. et al. (2019), The Gene Ontology Resource: 20 years and still GOing strong, Nucleic Acids Research 47 (D1), D330-D338.

Carroll, S.B. (2008), Evo-Devo and an Expanding Evolutionary Synthesis: A Genetic Theory of Morphological Evolution, Cell 134 (1), 25-36.

Carson, F.L. (1997), Histotechnology: a self-instructional text, 2nd edition, American Society of Clinical Pathologists, Hong Kong.

Castoe, T. a, A.P.J. de Koning and D.D. Pollock (2010), Adaptive molecular convergences, Communicative \& Integrative Biology 3 (1), 67-69.

Chambers, J., T. Hastie and D. Pregibon (1990), Statistical Models in S, Compstat, Physica-Verlag HD, Heidelberg, 317-321.

Chanarat, S. and K. Sträßer (2013), Splicing and beyond: The many faces of the Prp19 complex, Biochimica et Biophysica Acta - Molecular Cell Research 1833 (10), 2126-2134.

Chaney, R.W., P.C. Kaisen and H.L. Maison (1936), A Pleistocene flora from Fairbanks, Alaska. American Museum novitates, Amer. Mus. Nat. Hist. Novitates.

Charif, D. and J.R. Lobry (2007), SeqinR 1.0-2: A Contributed Package to the R Project for Statistical Computing Devoted to Biological Sequences Retrieval and Analysis, 207-232.

Cheng, J.B. and D.W. Russell (2004), Mammalian wax biosynthesis: II. Expression cloning of wax synthase cDNAs encoding a member of the acyltransferase enzyme family, Journal of Biological Chemistry 279 (36), 37798-37807.

Clémencon, H. (2012), Cytology and Plectology of the Hymenomycetes, 2nd edition, Gebr. Borntraeger Verlagsbuchhandlung, Stuttgart.

Colosimo, P.F. et al. (2005), Widespread parallel evolution in sticklebacks by repeated fixation of ectodysplasin alleles, Science 307 (5717), 1928-1933.

Condamine, F.L., A. Nel, P. Grandcolas and F. Legendre (2020), Fossil and phylogenetic analyses reveal recurrent periods of diversification and extinction in dictyopteran insects, Cladistics, 1-19.

Core Team R (2018), R: A language and environment for statistical computing.

Cornwell, W. and S. Nakagawa (2017), Phylogenetic comparative methods, Current Biology 27 (9), R333-R336.

Cowin, S.C. (2000), How is a tissue built?, Journal of Biomechanical Engineering 122 (6), 553-569.

Daher, F.B. and S.A. Braybrook (2015), How to let go: Pectin and plant cell adhesion, Frontiers in Plant Science 6 (JULY), 1-8.

Daskalov, A., J. Heller, S. Herzog and N.L. Glass (2017), Molecular Mechanisms Regulating Cell Fusion and Heterokaryon Formation in Filamentous Fungi, The Fungal Kingdom (20), 215-229.

Dighton, J. (2016), Fungi in Ecosystem Processes, Second Edi edition, CRC Press.

Dobin, A. et al. (2013), STAR: Ultrafast universal RNA-seq aligner, Bioinformatics 29 (1), 15-21.

Doré, J. et al. (2015), Comparative genomics, proteomics and transcriptomics give new insight into the exoproteome of the basidiomycete Hebeloma cylindrosporum and its involvement in ectomycorrhizal symbiosis, New Phytologist 208 (4), 11691187. 
Dressaire, E., L. Yamada, B. Song and M. Roper (2016), Mushrooms use convectively created airflows to disperse their spores, Proceedings of the National Academy of Sciences of the United States of America 113 (11), 2833-2838.

Duringer, P. et al. (2006), The first fossil fungus gardens of Isoptera: Oldest evidence of symbiotic termite fungiculture (Miocene, Chad basin), Naturwissenschaften 93 (12), 610-615.

Eisen, J.A. and C.M. Fraser (2003), Phylogenomics: Intersection of evolution and genomics, Science 300 (5626), 1706-1707.

Elmqvist, T. and A.P. Cox (1996), The evolution of viviparity in flowering plants, Oikos 77, 3-9.

Espina, V. et al. (2006), Laser-capture microdissection, Nature Protocols 1 (2), 586603.

Evert, R.F. and S.E. Eichhorn (2006), Esau's Plant Anatomy: Meristems, Cells, and Tissues of the Plant Body: Their Structure, Function, and Development, 3rd edition, JohnWiley \& Sons, Inc., New Jersey.

Ewels, P., M. Magnusson, S. Lundin and M. Käller (2016), MultiQC: Summarize analysis results for multiple tools and samples in a single report, Bioinformatics 32 (19), 3047-3048.

Fischer, M.W.F. and N.P. Money (2010), Why mushrooms form gills: efficiency of the lamellate morphology, Fungal Biology 114 (1), 57-63.

Fitzjohn, R.G. (2012), Diversitree: Comparative phylogenetic analyses of diversification in R, Methods in Ecology and Evolution 3 (6), 1084-1092.

Fitzjohn, R.G., W.P. Maddison and S.P. Otto (2009), Estimating trait-dependent speciation and extinction rates from incompletely resolved phylogenies, Systematic Biology 58 (6), 595-611.

Fleißner, A., A.R. Simonin and N.L. Glass (2008), Cell fusion in the filamentous fungus, Neurospora crassa, Methods in Molecular Biology 475, 21-38.

Floudas, D. et al. (2012), The Paleozoic origin of enzymatic lignin decomposition reconstructed from 31 fungal genomes., Science 336 (6089), 1715-9.

del Fresno, C., S. Iborra, P. Saz-Leal, M. Martínez-López and D. Sancho (2018), Flexible signaling of Myeloid C-type lectin receptors in immunity and inflammation, Frontiers in Immunology 9 (APR), 1-13.

Fricker, M.D., L.L.M. Heaton, N.S. Jones and L. Boddy (2017), The Mycelium as a Network, The Fungal Kingdom (May), 335-367.

Fu, C., P. Iyer, A. Herkal, J. Abdullah, A. Stout and S.J. Free (2011), Identification and characterization of genes required for cell-to-cell fusion in neurospora crassa, Eukaryotic Cell 10 (8), 1100-1109.

Gargano, M.L. et al. (2017), Medicinal mushrooms: Valuable biological resources of high exploitation potential, Plant Biosystems 151 (3), 548-565.

Gaude, N., S. Bortfeld, N. Duensing, M. Lohse and F. Krajinski (2012), Arbusculecontaining and non-colonized cortical cells of mycorrhizal roots undergo extensive and specific reprogramming during arbuscular mycorrhizal development, Plant Journal 69 (3), 510-528.

Gaya, E. et al. (2015), The adaptive radiation of lichen-forming Teloschistaceae is associated with sunscreening pigments and a bark-to-rock substrate shift, Proceedings of the National Academy of Sciences 112 (37), 11600-11605.

Geweke, J. (1992), Evaluating the accuracy of sampling-based approaches to the calculation of posterior moments, Bayesian Statistics 4, 169-193.

Girometta, C. et al. (2019), Physico-mechanical and thermodynamic properties of mycelium-based biocomposites: A review, Sustainability (Switzerland) 11 (2). 
Gould, S.J. and E.S. Vrba (1982), Exaptation — a Missing Term in the Science of Form, Paleobiology 8 (1), 4-15.

Grigoriev, I. V. et al. (2014), MycoCosm portal: Gearing up for 1000 fungal genomes, Nucleic Acids Research 42 (D1), 699-704.

Grimm, D. and H.A.B. Wösten (2018), Mushroom cultivation in the circular economy, Applied Microbiology and Biotechnology 102 (18), 7795-7803.

De Groot, P.W.J., R.T.P. Roeven, L.J.L.D. Van Griensven, J. Visser and P.J. Schaap (1999), Different temporal and spatial expression of two hydrophobin-encoding genes of the edible mushroom Agaricus bisporus, Microbiology 145 (5), 11051113.

Hacquard, S., E. Tisserant, A. Brun, V. Legué, F. Martin and A. Kohler (2013), Laser microdissection and microarray analysis of Tuber melanosporum ectomycorrhizas reveal functional heterogeneity between mantle and Hartig net compartments, Environmental Microbiology 15 (6), 1853-1869.

Halbwachs, H. and C. Bässler (2015), Gone with the wind - a review on basidiospores of lamellate agarics, Mycosphere 6 (1), 78-112.

Halbwachs, H., J. Simmel and C. Bässler (2016), Tales and mysteries of fungal fruiting: How morphological and physiological traits affect a pileate lifestyle, Fungal Biology Reviews 30 (2), 36-61.

Hamann, A., D. Brust and H.D. Osiewacz (2008), Apoptosis pathways in fungal growth, development and ageing, Trends in Microbiology 16 (6), 276-283.

Hammad, F., J. Ji, R. Watling and D. Moore (1993), Cell population dynamics in Coprinus cinereus: co-ordination of cell inflation throughout the maturing basidiome, Mycological Research 97 (3), 269-274.

Hardwick, J.M. (2018), Do fungi undergo apoptosis-like programmed cell death?, mBio 9 (4), 1-9.

Heckman, C.A., S.D. Pelok, S.A. Kimpel and L.-C. Wu (1989), Scanning Electron Microscope Studies on Fruitbody Primordium Formation in Agaricus Bisporus , Mycologia 81 (5), 717-727.

Helmstetter, A.J., A.S.T. Papadopulos, J. Igea, T.J.M. Van Dooren, A.M. Leroi and V. Savolainen (2016), Viviparity stimulates diversification in an order of fish, Nature Communications 7, 11271.

Hemsworth, G.R., G.J. Davies and P.H. Walton (2013), Recent insights into coppercontaining lytic polysaccharide mono-oxygenases, Current Opinion in Structural Biology 23 (5), 660-668.

Henderson, L.E. and I.K. Ross (1983), Ultrastructural Studies of Vegetative and Fruiting Mycelia of Coprinus congregatus, Mycologia 75 (4), 634.

Henehan, M.J. et al. (2019), Rapid ocean acidification and protracted Earth system recovery followed the end-Cretaceous Chicxulub impact, Proceedings of the National Academy of Sciences of the United States of America 116 (45), 22500 22504.

Hibbett, D.S. et al. (2014), 14 Agaricomycetes, Systematics and Evolution, Springer Berlin Heidelberg, Berlin, Heidelberg, 373-429.

Hibbett, D.S. (2007), After the gold rush, or before the flood? Evolutionary morphology of mushroom-forming fungi (Agaricomycetes) in the early 21 st century, Mycological Research 111 (9), 1001-1018.

Hibbett, D.S. et al. (2007), A higher-level phylogenetic classification of the Fungi., Mycological research 111 (Pt 5), 509-47.

Hibbett, D.S. (2006), A phylogenetic overview of the Agaricomycotina, Mycologia 98 (6), 917-925. 
Hibbett, D.S. (2004), Trends in Morphological Evolution in Homobasidiomycetes Inferred Using Maximum Likelihood: A Comparison of Binary and Multistate Approaches, Systematic Biology 53 (6), 889-903.

Hibbett, D.S. and M. Binder (2002), Evolution of complex fruiting-body morphologies in homobasidiomycetes, Proceedings of the Royal Society B: Biological Sciences 49 (191), 1963-1969.

Hibbett, D.S., D. Grimaldi and M.J. Donoghue (1997), Fossil mushrooms from Miocene and Cretaceous ambers and the evolution of homobasidiomycetes, American Journal of Botany 84 (7), 981-991.

Hines, M.A. and L.A. Taneyhill (2019), Adherens Junctions in Development, Reference Module in Life Sciences, Elsevier.

Ho, S.Y.W. and S. Duchêne (2014), Molecular-clock methods for estimating evolutionary rates and timescales, Molecular Ecology 23 (24), 5947-5965.

Höhna, S. (2015), The time-dependent reconstructed evolutionary process with a keyrole for mass-extinction events, Journal of Theoretical Biology 380, 321-331.

Höhna, S. (2013), Fast simulation of reconstructed phylogenies under global timedependent birth-death processes, Bioinformatics 29 (11), 1367-1374.

Höhna, S., M.R. May and B.R. Moore (2016), TESS: An R package for efficiently simulating phylogenetic trees and performing Bayesian inference of lineage diversification rates, Bioinformatics 32 (5), 789-791.

Höhna, S., M.R. May and B.R. Moore (2015), Phylogeny Simulation and Diversification Rate Analysis with TESS.

Holland, B.R., S. Ketelaar-Jones, A.R. O'Mara, M.D. Woodhams and G.J. Jordan (2020), Accuracy of ancestral state reconstruction for non-neutral traits, Scientific Reports 10 (1), 1-10.

Van Holle, S. and J.M. Van Damme (2018), Signaling through plant lectins: modulation of plant immunity and beyond, Biochemical Society Transactions 46 (2), 217-233.

Huelsenbeck, J.P., R. Nielsen and J.P. Bollback (2003), Stochastic mapping of morphological characters, Systematic Biology 52 (2), 131-158.

Hyde, K.D. et al. (2019), The amazing potential of fungi: 50 ways we can exploit fungi industrially, Fungal Diversity 97 (1), 1-136.

Ignatiadis, N., B. Klaus, J.B. Zaugg and W. Huber (2016), Data-driven hypothesis weighting increases detection power in genome-scale multiple testing, Nature Methods 13 (7), 577-580.

Jablonski, D. (1994), Extinctions in the fossil record, Philosophical Transactions Royal Society of London, B 344 (1307), 11-17.

Jaeger, R.J.R. and P. Spiteller (2010), Mycenaaurin A, an antibacterial polyene pigment from the fruiting bodies of mycena aurantiomarginata, Journal of Natural Products 73 (8), 1350-1354.

Jones, M., A. Mautner, S. Luenco, A. Bismarck and S. John (2020), Engineered mycelium composite construction materials from fungal biorefineries: A critical review, Materials and Design 187, 108397.

Jousselin, E., S. Van Noort and J.M. Greeff (2004), Labile male morphology and intraspecific male polymorphism in the Philotrypesis fig wasps, Molecular Phylogenetics and Evolution 33 (3), 706-718.

Kamada, T., H. Sano, T. Nakazawa and K. Nakahori (2010), Regulation of fruiting body photomorphogenesis in Coprinopsis cinerea, Fungal Genet Biol 47 (11), 917921.

Kamada, T. and M. Tsuru (1993), The onset of the helical arrangement of chitin microfibrils in fruit-body development of Coprinus cinereus, Mycological 
Research 97 (7), 884-888.

Kanehisa, M. (2000), KEGG: Kyoto Encyclopedia of Genes and Genomes, Nucleic Acids Research 28 (1), 27-30.

Kanehisa, M., Y. Sato, M. Furumichi, K. Morishima and M. Tanabe (2019), New approach for understanding genome variations in KEGG, Nucleic Acids Research 47 (D1), D590-D595.

Kass, R.E. and A.E. Raftery (1995), Bayes factors, Journal of the American Statistical Association 90 (430), 773-795.

Kauffman, E.G. and M.B. Hart (1996), Cretaceous Bio-Events, Global Events and Event Stratigraphy in the Phanerozoic, Springer Berlin Heidelberg, Berlin, Heidelberg, 285-312.

Kazmierczak, P., H.K. Dae, M. Turina and N.K. Van Alfen (2005), A hydrophobin of the chestnut blight fungus, Cryphonectria parasitica, is required for stromal pustule eruption, Eukaryotic Cell 4 (5), 931-936.

Khan, A., M. McQuilken and A.S. Gladfelter (2015), Septins and Generation of Asymmetries in Fungal Cells, Annual Review of Microbiology 69 (1), 487-503.

Khan, D. et al. (2015), Transcriptome atlas of the Arabidopsis funiculus - a study of maternal seed subregions, The Plant Journal, n/a-n/a.

King, R.W., P.K. Jackson and M.W. Kirschner (1994), Mitosis in Transition Review, Cell 79, 563-571.

Kiss, E. et al. (2019), Comparative genomics reveals the origin of fungal hyphae and multicellularity, Nature Communications 10 (1), 4080.

Knobloch, E. and F. Kotlaba (1994), Trametites eocenicus, a new fossil polypore from the Bohemian Eocene, Czech mycology 47 (3), 207-214.

Knoll, A.H. (2011), The Multiple Origins of Complex Multicellularity, Annual Review of Earth and Planetary Sciences 39 (1), 217-239.

Knoll, A.H. and D. Hewitt (2011), Phylogenetic, Functional, and Geological Perspectives on Complex Multicellularity, The Major Transitions in Evolution Revisited, The MIT Press, Cambridge, 251-270.

Knoll, A.H. and D.J.G. Lahr (2016), Fossils, Feeding and the Evolution of Complex Multicellularity, in: Niklas, K. J., S. A. Newman and J. T. Bonner (eds.), Multicellularity: origins and evolution, vol. The Vienna, MIT Press.

Kohler, A. et al. (2015), Convergent losses of decay mechanisms and rapid turnover of symbiosis genes in mycorrhizal mutualists, Nature Genetics 47 (4), 410-415.

Krah, F.-S., C. Bässler, C. Heibl, J. Soghigian, H. Schaefer and D.S. Hibbett (2018), Evolutionary dynamics of host specialization in wood-decay fungi, BMC Evolutionary Biology 18 (1), 119.

Kristensen, D.M., Y.I. Wolf, A.R. Mushegian and E. V. Koonin (2011), Computational methods for Gene Orthology inference, Briefings in Bioinformatics 12 (5), 379391.

Krizsán, K. et al. (2019), Transcriptomic atlas of mushroom development reveals conserved genes behind complex multicellularity in fungi, Proceedings of the National Academy of Sciences 116 (15), 7409-7418.

Kück, U., A.M. Beier and I. Teichert (2016), The composition and function of the striatin-interacting phosphatases and kinases (STRIPAK) complex in fungi, Fungal Genetics and Biology 90, 31-38.

Kües, U. (2000), Life history and developmental processes in the basidiomycete Coprinus cinereus., Microbiology and molecular biology reviews : MMBR 64 (2), 316-353.

Kües, U. and M. Navarro-González (2015), How do Agaricomycetes shape their 
fruiting bodies? 1. Morphological aspects of development, Fungal Biology Reviews 29 (2), 63-97.

Künzler, M. (2018), How fungi defend themselves against microbial competitors and animal predators, PLOS Pathogens 14 (9), e1007184.

Lang, D. and S.A. Rensing (2015), The Evolution of Transcriptional Regulation in the Viridiplantae and its Correlation with Morphological Complexity, Evolutionary Transitions to Multicellular Life, 301-333.

Lartillot, N., T. Lepage and S. Blanquart (2009), PhyloBayes 3: A Bayesian software package for phylogenetic reconstruction and molecular dating, Bioinformatics 25 (17), 2286-2288.

Lawrence, M. et al. (2013), Software for Computing and Annotating Genomic Ranges, PLoS Computational Biology 9 (8), 1-10.

Lemey, P., M. Salemi and A.-M. Vandamme (2009), The phyogenetic handbook, second edition, Cambridge University Press, New York.

Lepage, B.A., R.S. Currah, R.A. Stockey and G.W. Rothwell (1997), Fossil ectomycorrhizae from the middle Eocene, American Journal of Botany 84 (3), 410-412.

Lewin, H.A. et al. (2018), Earth BioGenome Project: Sequencing life for the future of life, Proceedings of the National Academy of Sciences of the United States of America 115 (17), 4325-4333.

Lewis, P.O. (2001), A likelihood approach to estimating phylogeny from discrete morphological character data, Systematic Biology 50 (6), 913-925.

Li, F. et al. (2008), Identification of the wax ester synthase/acyl-coenzyme a:diacylglycerol acyltransferase WSD1 required for stem wax ester biosynthesis in Arabidopsis, Plant Physiology 148 (1), 97-107.

Li, R. and A.W. Murray (1991), Feedback control of mitosis in budding yeast, Cell 66 (3), 519-531.

Limpens, E. (2020), Laser microdissection of arbuscular mycorrhiza, The Model Legume Medicago truncatula, 501-512.

Linder, M.B. (2009), Hydrophobins: Proteins that self assemble at interfaces, Current Opinion in Colloid and Interface Science 14 (5), 356-363.

Linder, M.B., G.R. Szilvay, T. Nakari-Setälä and M.E. Penttilä (2005), Hydrophobins: The protein-amphiphiles of filamentous fungi, FEMS Microbiology Reviews 29 (5), 877-896.

Lipke, P.N. (2018), What we do not know about fungal cell adhesion molecules, Journal of Fungi 4 (2).

Liu, Y.J. and B.D. Hall (2004), Body plan evolution of ascomycetes, as inferred from an RNA polymerase II phylogeny, Proceedings of the National Academy of Sciences of the United States of America 101 (13), 4507-4512.

Longrich, N.R., T. Tokaryk and D.J. Field (2011), Mass extinction of birds at the cretaceous-paleogene (K-Pg) boundary, Proceedings of the National Academy of Sciences of the United States of America 108 (37), 15253-15257.

Lord, K.M. and N.D. Read (2011), Perithecium morphogenesis in Sordaria macrospora, Fungal Genet Biol 48 (4), 388-399.

Louca, S. and M. Doebeli (2018), Efficient comparative phylogenetics on large trees, Bioinformatics 34 (6), 1053-1055.

Love, M.I., W. Huber and S. Anders (2014), Moderated estimation of fold change and dispersion for RNA-seq data with DESeq2, Genome Biology 15 (12), 1-21.

Loytynoja, A. and N. Goldman (2005), From The Cover: An algorithm for progressive multiple alignment of sequences with insertions, Proceedings of the National 
Academy of Sciences 102 (30), 10557-10562.

Lu, B.C. (1991), Cell degeneration and gill remodelling during basidiocarp development in the fungus Coprinus cinereus , Canadian Journal of Botany 69 (6), 1161-1169.

Lugones, L.G., H.A.B. Wösten, K.U. Birkenkamp, K.A. Sjollema, J. Zagers and J.G.H. Wessels (1999), Hydrophobins line air channels in fruiting bodies of Schizophyllum commune and Agaricus bisporus, Mycological Research 103 (5), 635-640.

Luti, S., L. Sella, A. Quarantin, L. Pazzagli and I. Baccelli (2020), Twenty years of research on cerato-platanin family proteins: clues, conclusions, and unsolved issues, Fungal Biology Reviews 34 (1), 13-24.

Lutzoni, F. et al. (2018), Contemporaneous radiations of fungi and plants linked to symbiosis, Nature Communications 9 (1), 5451.

Macosko, E.Z. et al. (2015), Highly Parallel Genome-wide Expression Profiling of Individual Cells Using Nanoliter Droplets, CELL 161 (5), 1202-1214.

Maddison, W.P., P.E. Midford and S.P. Otto (2007), Estimating a binary character's effect on speciation and extinction, Systematic Biology 56 (5), 701-710.

Magallon-Puebla, S. and S.R.S. Cevallos-Ferriz (1993), A Fossil Earthstar (Geasteraceae; Gasteromycetes) from the Late Cenozoic of Puebla, Mexico, American Journal of Botany 80 (10), 1162-1167.

Mann, F., W.A. Harris and C.E. Holt (2004), New views on retinal axon development: a navigation guide, The International Journal of Developmental Biology 48 (8-9), 957-964.

Matheny, P.B. et al. (2006), Major clades of Agaricales: A multilocus phylogenetic overview, Mycologia 98 (6), 982-995.

Matthews, T.R. and D.J. Niederpruem (1973), Differentiation in Coprinus lagopus, Archives of Microbiology 88, 169-180.

May, M.R., S. Höhna and B.R. Moore (2016), A Bayesian approach for detecting the impact of mass-extinction events on molecular phylogenies when rates of lineage diversification may vary, Methods in Ecology and Evolution 7 (8), 947-959.

McKenna, D.D., A.S. Sequeira, A.E. Marvaldi and B.D. Farrell (2009), Temporal lags and overlap in the diversification of weevils and flowering plants, Proceedings of the National Academy of Sciences 106 (17), 7083-7088.

Merényi, Z. et al. (2020), Unmatched level of molecular convergence among deeply divergent complex multicellular fungi, Molecular Biology and Evolution, 549758.

Mitchell, A.L. et al. (2019), InterPro in 2019: improving coverage, classification and access to protein sequence annotations, Nucleic Acids Research 47 (D1), D351D360.

Mitchell, K.F., R. Zarnowski and D.R. Andes (2016), Fungal Super Glue: The Biofilm Matrix and Its Composition, Assembly, and Functions, PLoS Pathogens 12 (9), 16.

Moen, D. and H. Morlon (2014), Why does diversification slow down?, Trends in Ecology and Evolution 29 (4), 190-197.

Moore, D. (1995), Tissue Formation, The Growing Fungus, Springer Netherlands, Dordrecht, 423-465.

Moore, D., L.A. Casselton, D.A. Wood and J.C. Frankland (1984), Developmental Biology of Higher Fungi, Cambridge University Press, Cambridge.

Moore, D., M. Elhiti and R. Butler (1979), Morphogenesis of the Carpophore of Coprinus cinereus, New Phytologist 83 (3), 695-722.

Moore, D., D.G. Robson and P.J.A. Trinci (2011), 21st Century Guidebook to fungi, 
Cambridge University Press, New York.

Muraguchi, H. et al. (2015), Strand-Specific RNA-Seq Analyses of Fruiting Body Development in Coprinopsis cinerea, Plos One 10 (10), e0141586.

Muraguchi, H. et al. (2008), The exp1 gene essential for pileus expansion and autolysis of the inky cap mushroom Coprinopsis cinerea (Coprinus cinereus) encodes an HMG protein, Fungal Genetics and Biology 45 (6), 890-896.

Muraguchi, H. and T. Kamada (2000), A mutation in the eln2 gene encoding a cytochrome $\mathrm{P} 450$ of Coprinus cinereus affects mushroom morphogenesis, Fungal Genetics and Biology 29 (1), 49-59.

Nagy, L.G. et al. (2016), Comparative genomics of early-diverging mushroom-forming fungi provides insights into the origins of lignocellulose decay capabilities, Molecular Biology and Evolution 33 (4), 959-970.

Nagy, L.G. et al. (2014), Latent homology and convergent regulatory evolution underlies the repeated emergence of yeasts, Nature Communications 5 (1), 4471.

Nagy, L.G. et al. (2012), The evolution of defense mechanisms correlate with the explosive diversification of autodigesting coprinellus mushrooms (agaricales, fungi), Systematic Biology 61 (4), 595-607.

Nagy, L.G., G.M. Kovács and K. Krizsán (2018), Complex multicellularity in fungi: evolutionary convergence, single origin, or both?, Biological Reviews.

Nagy, L.G., R. Tóth, E. Kiss, J. Slot, A. Gácser and G.M. Kovács (2017), Six Key Traits of Fungi: Their Evolutionary Origins and Genetic Bases, Microbiology Spectrum 5 (4).

Nagy, L.G., T. Varga, Á. Csernetics and M. Virágh (2020), Fungi took a unique evolutionary route to multicellularity: Seven key challenges for fungal multicellular life, Fungal Biology Reviews (xxxx).

Nakamori, T. and A. Suzuki (2007), Defensive role of cystidia against Collembola in the basidiomycetes Russula bella and Strobilurus ohshimae, Mycological Research 111 (11), 1345-1351.

Naranjo-Ortiz, M.A. and T. Gabaldón (2019), Fungal evolution: major ecological adaptations and evolutionary transitions, Biological Reviews, brv.12510.

Nascimento, F.F., M. Dos Reis and Z. Yang (2017), A biologist's guide to Bayesian phylogenetic analysis, Nature Ecology and Evolution 1 (10), 1446-1454.

National Research Council (1982), Climate in Earth History, National Academies Press, Washington, D.C.

Near, T.J. and M.J. Sanderson (2004), Assessing the quality of molecular divergence time estimates by fossil calibrations and fossil-based model selection, Philosophical Transactions of the Royal Society B: Biological Sciences 359 (1450), 1477-1483.

Nee, S., E.C. Holmes, A. Rambaut and P.H. Harvey (1995), Inferring population history from molecular phylogenies, Philosophical Transactions of the Royal Society of London. Series B: Biological Sciences 349 (1327), 25-31.

Nelsen, M.P., R. Lücking, C.K. Boyce, H.T. Lumbsch and R.H. Ree (2020), The macroevolutionary dynamics of symbiotic and phenotypic diversification in lichens, Proceedings of the National Academy of Sciences (August), 202001913.

Niklas, K.J., S.A. Newman and R. Christine (2020), The many roads to and from multicellularity, Journal of Experimental Botany 71 (11), 3247-3253.

Niklas, K.J., B.H. Tiffney and A.H. Knoll (1983), Patterns in vascular land plant diversification, Nature 303 (5918), 614-616.

Novacek, M.J. (1999), 100 million years of land vertebrate evolution: The cretaceousearly tertiary transition, Annals of the Missouri Botanical Garden 86 (2), 230-258. 
Ohtomo, Y., T. Kakegawa, A. Ishida, T. Nagase and M.T. Rosing (2014), Evidence for biogenic graphite in early archaean isua metasedimentary rocks, Nature Geoscience 7 (1), 25-28.

Orwin, K.H., M.U.F. Kirschbaum, M.G. St John and I.A. Dickie (2011), Organic nutrient uptake by mycorrhizal fungi enhances ecosystem carbon storage: A model-based assessment, Ecology Letters 14 (5), 493-502.

Ostrovsky, A.N., S. Lidgard, D.P. Gordon, T. Schwaha, G. Genikhovich and A. V. Ereskovsky (2016), Matrotrophy and placentation in invertebrates: a new paradigm, Biological reviews of the Cambridge Philosophical Society 91 (3), 673711.

Pagel, M. (1999), Inferring the historical patterns of biological evolution., Nature 401 (6756), 877-884.

Pagel, M. (1994), Detecting Correlated Evolution on Phylogenies: a General Method for the Comparative Analysis of Discrete Characters, Proceedings of the Royal Society of London 255, 37-45.

Pagel, M. and A. Meade (2007), BayesTraits, Computer program and documentation available at http://www. evolution. rdg. ac. uk/BayesTraits. html, 1216-1223.

Paradis, E., J. Claude and K. Strimmer (2004), APE: Analyses of phylogenetics and evolution in R language, Bioinformatics 20 (2), 289-290.

Parins-Fukuchi, C. (2018), Use of Continuous Traits Can Improve Morphological Phylogenetics, Systematic Biology 67 (2), 328-339.

Perillo, N.L., M.E. Marcus and L.G. Baum (1998), Galectins: Versatile modulators of cell adhesion, cell proliferation, and cell death, Journal of Molecular Medicine 76 (6), 402-412.

Picelli, S., Å.K. Björklund, O.R. Faridani, S. Sagasser, G. Winberg and R. Sandberg (2013), Smart-seq 2 for sensitive full-length transcriptome profiling in single cells, Nature Methods 10 (11), 1096-1098.

Picelli, S., O.R. Faridani, Å.K. Björklund, G. Winberg, S. Sagasser and R. Sandberg (2014), Full-length RNA-seq from single cells using Smart-seq2, Nature Protocols 9 (1), 171-181.

Plaza, D.F., C.W. Lin, N.S. van der Velden, M. Aebi and M. Kunzler (2014), Comparative transcriptomics of the model mushroom Coprinopsis cinerea reveals tissue-specific armories and a conserved circuitry for sexual development, BMC Genomics 15, 492.

Plummer, M., N. Best, K. Cowles and K. Vines (2006), CODA: convergence diagnosis and output analysis for MCMC, R News 6 (March), 7-11.

Pöggeler, S., M. Nowrousian, I. Teichert, A. Beier and U. Kück (2018), Fruiting-Body Development in Ascomycetes, Physiology and Genetics, Springer International Publishing, Cham, 1-56.

Pohleven, J. et al. (2011), Basidiomycete Clitocybe nebularis is rich in lectins with insecticidal activities, Applied Microbiology and Biotechnology 91 (4), 11411148.

Pohleven, J. et al. (2009), Purification, characterization and cloning of a ricin B-like lectin from mushroom Clitocybe nebularis with antiproliferative activity against human leukemic T cells, Biochimica et Biophysica Acta - General Subjects 1790 (3), 173-181.

Poinar, G.O. (2014), Bird's nest fungi (Nidulariales: Nidulariaceae) in Baltic and Dominican amber, Fungal Biology 118 (3), 325-329.

Poinar, G.O. and R. Buckley (2007), Evidence of mycoparasitism and hypermycoparasitism in Early Cretaceous amber, Mycological Research 111 (4), 
503-506.

Polz, M.F. and C.M. Cavanaugh (1998), Bias in template-to-product ratios in multitemplate PCR, Applied and Environmental Microbiology 64 (10), 37243730.

Pyron, R.A. and F.T. Burbrink (2014), Early origin of viviparity and multiple reversions to oviparity in squamate reptiles, Ecology Letters 17 (1), 13-21.

Rabosky, D.L. (2014), Automatic detection of key innovations, rate shifts, and diversity-dependence on phylogenetic trees, PLoS ONE 9 (2).

Rabosky, D.L. et al. (2014), BAMMtools: An R package for the analysis of evolutionary dynamics on phylogenetic trees, Methods in Ecology and Evolution 5 (7), 701-707.

Reichhardt, C., J.A.G. Ferreir, L.M. Joubert, K. V. Clemons, D.A. Stevens and L. Cegelski (2015), Analysis of the Aspergillus fumigatus biofilm extracellular matrix by solid-state nuclear magnetic resonance spectroscopy, Eukaryotic Cell 14 (11), 1064-1072.

Reid, L.H. (2005), Proposed methods for testing and selecting the ERCC external RNA controls, BMC Genomics 6 (June 2004), 1-18.

Reijnders, A.F.M. (1979), Developmental anatomy of Coprinus, Persoonia 10 (3), $383-$ 424.

Reijnders, A.F.M. (1948), Etudes sur le développement et l'organisation histologiques des carpophores dans les Agaricales, Recueil Travaux Botaniques Néerlandais 41, 213-396.

Reijnders, A.F.M. and D. Moore (1985), Developmental biology of agarics - an overview, Developmental biology of higher fungi, Cambridge University Press, Cambridge, 581-595.

Reijnders, M. (1977), The histogenesis of Bulb and trama tissue of the higher Basidiomycetes and its phylogenetic implications, Persoonia 9 (3), 329-361.

Revell, L.J. (2012), phytools: An R package for phylogenetic comparative biology (and other things), Methods in Ecology and Evolution 3 (2), 217-223.

Ribeiro, E., A.M. Davis, R.A. Rivero-Vega, G. Ortí and R. Betancur (2018), PostCretaceous bursts of evolution along the benthic-pelagic axis in marine fishes, Proceedings of the Royal Society B: Biological Sciences 285 (1893).

Riquelme, M. et al. (2018), Fungal Morphogenesis, from the Polarized Growth of Hyphae to Complex Reproduction and Infection Structures, Microbiology and Molecular Biology Reviews 82 (2), 1-47.

Risso, D., K. Schwartz, G. Sherlock and S. Dudoit (2011), GC-Content Normalization for RNA-Seq Data, BMC Bioinformatics 12 (1), 480.

Robinson, M.D., D.J. McCarthy and G.K. Smyth (2010), edgeR: a Bioconductor package for differential expression analysis of digital gene expression data., Bioinformatics (Oxford, England) 26 (1), 139-140.

Robson, S.K.A., R.J. Kohout, A.T. Beckenbach and C.S. Moreau (2015), Evolutionary transitions of complex labile traits: Silk weaving and arboreal nesting in Polyrhachis ants, Behavioral Ecology and Sociobiology 69 (3), 449-458.

Rodriques, S.G. et al. (2019), Slide-seq: A scalable technology for measuring genomewide expression at high spatial resolution, Science 363 (6434), 1463-1467.

Roger, A.J., O. Sandblom, W.F. Doolittle and H. Philippe (1999), An evaluation of elongation factor $1 \alpha$ as a phylogenetic marker for eukaryotes, Molecular Biology and Evolution 16 (2), 218-233.

Rokas, A. (2008), The Origins of Multicellularity and the Early History of the Genetic Toolkit For Animal Development, Annual Review of Genetics 42 (1), 235-251. 
Royse, D.J., J. Baars and Q. Tan (2017), Current Overview of Mushroom Production in the World, Edible and Medicinal Mushrooms 2010, 5-13.

Ruiz-Trillo, I. et al. (2007), The origins of multicellularity: a multi-taxon genome initiative, Trends in Genetics 23 (3), 113-118.

Sabotič, J., R.A. Ohm and M. Künzler (2016), Entomotoxic and nematotoxic lectins and protease inhibitors from fungal fruiting bodies, Applied Microbiology and Biotechnology 100 (1), 91-111.

Saliba, A.E.A.-E., A.J. Westermann, S.A. Gorski and J. Vogel (2014), Single-cell RNAseq: advances and future challenges, Nucleic Acids Research 42 (14), 8845-8860.

Sammer, D., K. Krause, M. Gube, K. Wagner and E. Kothe (2016), Hydrophobins in the life cycle of the ectomycorrhizal basidiomycete Tricholoma vaccinum, PLoS ONE 11 (12), 1-20.

Sánchez-Garcia, M. et al. (2017), Is the switch to an ectomycorrhizal state an evolutionary key innovation in mushroom-forming fungi? A case study in the Tricholomatineae (Agaricales), Evolution 71 (1), 51-65.

Sánchez-García, M., M. Ryberg, K.K. Faheema, T. Varga, L.G. Nagy and D.S. Hibbett (2020), Fruiting body form, not nutritional mode, is the major driver of diversification in mushroom-forming fungi based on an 8400-species megaphylogeny, Proceedings of the National Academy of Sciences, under revision.

Sanderson, M.J. (2003), r8s: Inferring absolute rates of molecular evolution and divergence times in the absence of a molecular clock, Bioinformatics 19 (2), 301302.

Sanderson, M.J. (2002), Estimating absolute rates of molecular evolution and divergence times: a penalized likelihood approach., Molecular biology and evolution 19 (1), 101-109.

Sato, H. and H. Toju (2019), Timing of evolutionary innovation: scenarios of evolutionary diversification in a species-rich fungal clade, Boletales, New Phytologist 222 (4), 1924-1935.

Schliep, K.P. (2011), phangorn: phylogenetic analysis in R., Bioinformatics (Oxford, England) 27 (4), 592-593.

Schmid, K.M. (2016), Lipid Metabolism in Plants, Sixth Edit edition, Elsevier.

Schmitt, I., R. del Prado, M. Grube and H.T. Lumbsch (2009), Repeated evolution of closed fruiting bodies is linked to ascoma development in the largest group of lichenized fungi (Lecanoromycetes, Ascomycota), Molecular Phylogenetics and Evolution 52 (1), 34-44.

Scholey, J.M., D.J. Sharp and G.C. Rogers (2000), Microtubule motors in mitosis., Nature 407 (6800), 41-47.

Schulte, P. et al. (2010), The chicxulub asteroid impact and mass extinction at the cretaceous-paleogene boundary, Science 327 (5970), 1214-1218.

Sebe-Pedros, A., B.M. Degnan and I. Ruiz-Trillo (2017), The origin of Metazoa: a unicellular perspective, Nat Rev Genet 18 (8), 498-512.

de Sena-Tomás, C., M. Navarro-González, U. Kües and J. Pérez-Martín (2013), A DNA damage checkpoint pathway coordinates the division of dikaryotic cells in the ink cap mushroom Coprinopsis cinerea, Genetics 195 (1), 47-57.

Shioya, T. et al. (2013), The Coprinopsis cinerea septin Cc.Cdc3 is involved in stipe cell elongation, Fungal Genetics and Biology 58-59, 80-90.

Shubin, N., C. Tabin and S. Carroll (2009), Deep homology and the origins of evolutionary novelty, Nature 457 (7231), 818-823.

Shulse, C.N. et al. (2019), High-Throughput Single-Cell Transcriptome Profiling of 
Plant Cell Types, Cell Reports 27 (7), 2241-2247.e4.

Sipos, G. et al. (2017), Genome expansion and lineage-specific genetic innovations in the forest pathogenic fungi Armillaria, Nature Ecology \& Evolution 1 (12), 19311941.

Smith, M.R. (2016), Cord-forming Palaeozoic fungi in terrestrial assemblages, Botanical Journal of the Linnean Society 180 (4), 452-460.

Smith, S.Y., R.S. Currah and R.A. Stockey (2004), Cretaceous and Eocene poroid hymenophores from Vancouver Island, British Columbia, Mycologia 96 (1), 180186.

Sonawane, A.R. et al. (2017), Understanding Tissue-Specific Gene Regulation, Cell Reports 21 (4), 1077-1088.

Sprain, C.J., P.R. Renne, L. Vanderkluysen, K. Pande, S. Self and T. Mittal (2019), The eruptive tempo of deccan volcanism in relation to the cretaceous-paleogene boundary, Science 363 (6429), 866-870.

Stajich, J.E. et al. (2010), Insights into evolution of multicellular fungi from the assembled chromosomes of the mushroom Coprinopsis cinerea (Coprinus cinereus), Proceedings of the National Academy of Sciences of the United States of America 107 (26), 11889-11894.

Stamatakis, A. (2014), RAxML version 8: A tool for phylogenetic analysis and postanalysis of large phylogenies, Bioinformatics 30 (9), 1312-1313.

Stephens, M. (2017), False discovery rates: A new deal, Biostatistics 18 (2), 275-294.

Sullivan, R., J.E. Smith and N.J. Rowan (2006), Medicinal mushrooms and cancer therapy: Translating a traditional practice into Western medicine, Perspectives in Biology and Medicine 49 (2), 159-170.

Szathmáry, E. (2015), Toward major evolutionary transitions theory 2.0, Proceedings of the National Academy of Sciences 112 (33), 10104-10111.

Szathmáry, E. and J. Maynard Smith (1995), The major evolutionary transitions, Nature 374 (6519), 227-232.

Talavera, G. and J. Castresana (2007), Improvement of phylogenies after removing divergent and ambiguously aligned blocks from protein sequence alignments, Systematic Biology 56 (4), 564-577.

Tatusov, R.L. et al. (2003), The COG database: An updated vesion includes eukaryotes, BMC Bioinformatics 4, 1-14.

Tayyrov, A., S.S. Schmieder, S. Bleuler-Martinez, D.F. Plaza and M. Künzler (2018), Toxicity of Potential Fungal Defense Proteins towards the Fungivorous Nematodes Aphelenchus avenae and Bursaphelenchus okinawaensis, Applied and Environmental Microbiology 84 (23), 1-9.

Teichert, I., G. Wolff, U. Kück and M. Nowrousian (2012), Combining laser microdissection and RNA-seq to chart the transcriptional landscape of fungal development, BMC Genomics 13 (1), 511.

Tennant, J.P., P.D. Mannion and P. Upchurch (2016), Sea level regulated tetrapod diversity dynamics through the Jurassic/Cretaceous interval, Nature Communications 7.

Tennant, J.P., P.D. Mannion, P. Upchurch, M.D. Sutton and G.D. Price (2017), Biotic and environmental dynamics through the Late Jurassic-Early Cretaceous transition: evidence for protracted faunal and ecological turnover, Biological Reviews 92 (2), 776-814.

Thakare, D. et al. (2014), RNA-Seq analysis of laser-capture microdissected cells of the developing central starchy endosperm of maize, Genomics Data 2, 242-245.

Thiers, H.D. (1984), The Secotioid Syndrome, Mycologia 76 (1), 1-8. 
Thorne, J.L., H. Kishino and I.S. Painter (1998), Estimating the rate of evolution of the rate of molecular evolution, Molecular Biology and Evolution 15 (12), 1647-1657.

UCLA: Statistical Consulting Group (2020), Introduction to SAS, https://stats.idre.ucla.edu/r/library/r-library-contrast-coding-systems-forcategorical-variables/, retrieved 1.3.2020.

Uhlén, M., B.M. Hallström, C. Lindskog, A. Mardinoglu, F. Pontén and J. Nielsen (2016), Transcriptomics resources of human tissues and organs, Molecular Systems Biology 12 (4), 862.

Vajda, V. (2001), Indication of Global Deforestation at the Cretaceous-Tertiary Boundary by New Zealand Fern Spike, Science 294 (5547), 1700-1702.

Vajda, V. and S. McLoughlin (2004), Fungal Proliferation at the Cretaceous-Tertiary Boundary, Science 303 (5663), 1489.

van der Valk, P. and R. Marchant (1978), Hyphal ultrastructure in fruit-body primordia of the basidiomycetes Schizophyllum commune and Coprinus cinereus, Protoplasma 95 (1-2), 57-72.

Varga, T. et al. (2019), Megaphylogeny resolves global patterns of mushroom evolution, Nature Ecology \& Evolution 3 (4), 668-678.

Velíšek, J. and K. Cejpek (2011), Pigments of higher fungi: A review, Czech Journal of Food Sciences 29 (2), 87-102.

Velo-Antón, G., K.R. Zamudio and A. Cordero-Rivera (2012), Genetic drift and rapid evolution of viviparity in insular fire salamanders (Salamandra salamandra), Heredity 108 (4), 410-418.

Walser, P.J., U. Kües, M. Aebi and M. Künzler (2005), Ligand interactions of the Coprinopsis cinerea galectins, Fungal Genetics and Biology 42 (4), 293-305.

Wang, H., T.B. Ng and V.E.C. Ooi (1998), Lectins from mushrooms, Mycological Research 102 (8), 897-906.

Watling, R. (1985), Developmental characters of agarics, Developmental biology of higher fungi, Cambridge University Press, Cambridge, 281-310.

Werner, G.D.A., W.K. Cornwell, J.I. Sprent, J. Kattge and E.T. Kiers (2014), A single evolutionary innovation drives the deep evolution of symbiotic N2-fixation in angiosperms, Nature Communications 5 (May), 1-9.

Wessels, J.G.H. (1996), Fungal hydrophobins: Proteins that function at an interface, Trends in Plant Science 1 (1), 9-15.

Wickham, H. (2009), Ggplot2.

Wilf, P., K.R. Johnson and B.T. Huber (2003), Correlated terrestrial and marine evidence for global climate changes before mass extinction at the CretaceousPaleogene boundary, Proceedings of the National Academy of Sciences of the United States of America 100 (2), 599-604.

Wilson, A.W., M. Binder and D.S. Hibbett (2011), Effects of gasteroid fruiting body morphology on diversification rates in three independent clades of fungi estimated using binary state speciation and extinction analysis, Evolution 65 (5), 1305-1322.

Wilson, A.W., K. Hosaka and G.M. Mueller (2017), Evolution of ectomycorrhizas as a driver of diversification and biogeographic patterns in the model mycorrhizal mushroom genus Laccaria, New Phytologist 213 (4), 1862-1873.

Wojciechowska, I. (2017), The leather underground: Biofabrication offers new sources for fabrics, AATCC Review 17 (6), 18-23.

Wolf, J.B.W. (2013), Principles of transcriptome analysis and gene expression quantification: An RNA-seq tutorial, Molecular Ecology Resources 13 (4), 559572.

Xie, W., P.O. Lewis, Y. Fan, L. Kuo and M.H. Chen (2011), Improving marginal 
likelihood estimation for bayesian phylogenetic model selection, Systematic Biology 60 (2), 150-160.

Xie, Y., J. Chang and H.S. Kwan (2020), Carbon metabolism and transcriptome in developmental paths differentiation of a homokaryotic Coprinopsis cinerea strain, Fungal Genetics and Biology 143 (June), 103432.

Yang, Z. (2014), Molecular Evolution: A statistical approach, Oxford University Press.

Yang, Z. (2007), PAML 4: Phylogenetic analysis by maximum likelihood, Molecular Biology and Evolution 24 (8), 1586-1591.

Yu, D.Y., S.J. Cringle, C. Balaratnasingam, W.H. Morgan, P.K. Yu and E.N. Su (2013), Retinal ganglion cells: Energetics, compartmentation, axonal transport, cytoskeletons and vulnerability, Progress in Retinal and Eye Research 36, 217246.

Zhang, W. et al. (2016), Purification, characterization and function analysis of an extracellular $\beta$-glucosidase from elongating stipe cell walls in Coprinopsis cinerea, FEMS Microbiology Letters 363 (9), 1-9.

Zhao, R.-L. et al. (2017), A six-gene phylogenetic overview of Basidiomycota and allied phyla with estimated divergence times of higher taxa and a phyloproteomics perspective, Fungal Diversity 84 (1), 43-74.

Zhou, S., J. Zhang, F. Ma, C. Tang, Q. Tang and X. Zhang (2018), Investigation of lignocellulolytic enzymes during different growth phases of Ganoderma lucidum strain G0119 using genomic, transcriptomic and secretomic analyses, PLOS ONE 13 (5), e0198404.

Zhou, Y., W. Zhang, Z. Liu, J. Wang and S. Yuan (2015), Purification, characterization and synergism in autolysis of a group of 1,3-\$beta $\{\backslash \$\}$-glucan hydrolases from the pilei of Coprinopsis cinerea fruiting bodies, Microbiology (United Kingdom) 161 (10), 1978-1989.

Ziegenhain, C. et al. (2017), Comparative Analysis of Single-Cell RNA Sequencing Methods, Molecular Cell 65 (4), 631-643.e4. 


\section{Appendix}

\section{Appendix 1. Low-input RNA-seq protocol}

\section{A. Fixation \& Embedding of primordia}

\section{I) Preparing solutions}

1) Farmer's fixative

75\% Absolute Ethyl Alcohol, 25\% Glacial Acetic Acid, 20 $\mu 1 / \mathrm{ml}$ 2-Mercaptoethanol

2) PBS solution

1. Add 9 volume RNase free water to 1 volume 10xPBS

2. Add an appropriate volume ( $\sim 0.1 \% \mathrm{v} / \mathrm{v})$ DEPC to this solution, mix it for at least 2 hours at $37^{\circ} \mathrm{C}$ and then autoclave it for $15 \mathrm{~min}$.

3) Sucrose solutions

1. Dissolve sucrose in $1 \%$ PBS and add $20 \mu \mathrm{l} / \mathrm{ml}$ 2-Mercaptoethanol to it.

2. Prepare five types of solutions: $10 \% ; 30 \% ; 2: 1$ ratio of $10 \%$ and $30 \% ; 1: 1$ ratio of $10 \%$ and $30 \% ; 1: 2$ ratio of $10 \%$ and $30 \%$ (The concentrations are given in mass concentration $(\mathrm{g} / 100 \mathrm{ml}))$.

\section{II) Fixation and embedding}

1) Fill a $2 \mathrm{ml}$ Eppendorf Tube with $1,8 \mathrm{ml}$ Farmer's fixative prepared in section $\mathrm{A} / \mathrm{I}$.

2) Cut out the primordium with a small layer ( $\sim 1 \mathrm{~mm}$ thick) of agar and put immediately into the fixative by holding only the agar

3) Incubate the specimen in the fixative for 30 minutes while vacuuming it three times at -0.8 bar.

4) Change the fixative to $1.8 \mathrm{ml} 10 \%$ Sucrose solution and incubate at least 15 minutes or until the specimen sink to the bottom of the tube

5) Change the sucrose solution to $1.8 \mathrm{ml} \mathrm{2:1} \mathrm{ratio} \mathrm{of} 10 \%$ and $30 \%$ sucrose solution and incubate at least 15 minutes or until the specimen sink to the bottom of the tube

6) Change the sucrose solution to $1.8 \mathrm{ml} 1: 1$ ratio of $10 \%$ and $30 \%$ sucrose solution and incubate at least 15 minutes or until the specimen sink to the bottom of the tube 
7) Change the sucrose solution to $1.8 \mathrm{ml} \mathrm{1:2} \mathrm{ratio} \mathrm{of} 10 \%$ and $30 \%$ sucrose solution and incubate at least 15 minutes or until the specimen sink to the bottom of the tube

8) Change the sucrose solution to $1.8 \mathrm{ml} 30 \%$ sucrose solution and incubate at least 15 minutes or until the specimen sink to the bottom of the tube

9) Repeat the previous point two more times.

During the sucrose-infiltration vacuum can be applied as well

10) Gently pour out the sucrose solution with the specimen into a sterile Petri dish

11) Move the specimen out of the sucrose solution and put Histolab Cryomount on it until it entirely covers the specimen.

12) Place a cryo-mold or an equivalent tool (here a metal cylinder) on dry ice and place a layer of Cryomount on the bottom.

13) Using a forceps, place the specimen to the bottom of a metal cylinder containing unfrozen cryomount. Try not to touch the specimen. To do that, open the jaws of forceps to around $2 \mathrm{~mm}$. Adhesion of the cryo-mount to the jaws can help to keep the specimen in between the jaws.

14) Quickly orient the specimen with a needle.

16) Wait until the cryo-mount freeze enough to fix the orientation of the specimen

17) Add cryo-mount to the top.

18) Store the frozen specimen in a $-70^{\circ} \mathrm{C}$ freezer

\section{B. Cryo-sectioning}

\section{I) Preparing the cryostat, solutions and slides}

1) Cryostat:

- Wipe down the inside surface of the instrument with abs. Ethanol.

- Install a new microtome blade.

- Wipe down the outside surface of the instrument with RNase-ExitusPlus ${ }^{\mathrm{TM}}$

- UV sterilizer the inner surface of the instrument for 3 hours

2) Brushes

- Soak the head of the brushes into RNase-ExitusPlus ${ }^{\mathrm{TM}}$ for few minutes

- Rinse the brushes into RNAse free water three times

- Rinse the brushes into $100 \%$ ethanol twice 
- UV sterilize the brushes at least for three hours

3) PEN covered \& Poly-L-lysine coated slides

1. Treat the PEN surface with UV for at least 3 hours

This step is essential to improve adhesion!

2. Apply a sufficient volume ( 2-3 ml) of $0.01 \%$ Poly-L-lysine solution to cover the membrane surface.

Nail polish can be used to mark out the border of the membrane, it will help to keep the solution only on the membrane.

3. Incubate slide for $10 \mathrm{~min}$ at room temperature

4. Remove the Poly-L-lysine solution from the membrane by pipetting

5. Repeat the previous three steps again

Repeating the Poly-L-lysine treatment can improve the adhesion of samples

6. Allow air-drying of the slide

7. UV sterilize the Poly-L-lysine coated slides for 3 hours

4) Glass slides

Wipe down with $100 \%$ ethanol

5) Solutions for tissue section dehydration:

- Clean every staining jars by Excytus, then wash them with DEPC treated water

- Use 5 staining jars, every solution made of DEPC treated MiliQ water containing $5 \mu \mathrm{l} / \mathrm{ml}$ 2-mercaptoethanol:

○ Jar 1: $75 \%$ ethanol

○ Jar 2: DEPC treated MiliQ water

○ Jar 3: $75 \%$ ethanol

○ Jar 4: $95 \%$ ethanol

○ Jar 5: 100\% ethanol 


\section{II) Cryo-sectioning}

1) Take out the UV treated PEN slide and place them into a clean, closeable slide holder box.

2) Set the cryostat to $-20^{\circ} \mathrm{C}$, place specimens from section $\mathrm{A}$ inside, and let them equilibrate with the temperature of the cryostat (> 10 minutes).

3) Mount specimen to specimen holder.

4) Start sectioning the whole frozen block of the specimen and as soon as the tissue becomes visible, trim the block with a scalpel to remove unnecessary cryo-mount material around the tissue.

5) Cut $13 \mu \mathrm{m}$ thick section, and mount them towards a simple glass slide

6) Check the sections if they are the region of interest

7) Mount the following sections on the UV treated PEN slides prepared in section $B / I / 3$. If the slides are pre-warmed to $\sim 30-40^{\circ} \mathrm{C}$ the tissue section mounting is smoother

8) Once a slide is filled put it back into the slide holder box

\section{III) Washing and dehydrate tissue sections}

1) Place the slides with sections on them in the jar containing $75 \%$ ethanol (Jar 1) for 10 minutes

Adhesion of sections can be improved by a primer incubation in $75 \%$ ethanol for 10 minutes

2) Transfer the slides to the jar containing water (Jar 2) for 30 seconds

3) Transfer back the slides into the jar containing $75 \%$ ethanol (Jar 1) for 30 seconds

4) Repeat the 2-3 steps two more times

Step 4 is important to wash out all the mounting and sucrose solutions, which provides better performance through LCM!

5) Transfer the slides to the jar containing $75 \%$ ethanol (Jar 3) for 30 seconds

6) Transfer the slides to the jar containing 95\% ethanol (Jar 4) for 30 seconds

7) Transfer the slides to the jar containing $100 \%$ ethanol (Jar 5) for 30 seconds

8) Put the slides on a paper towel, let the ethanol evaporate on room temperature 
9) Transfer slides to a clean slide holder box.

\section{C) Laser Capture Microdissection (LCM)}

\section{Prepare $0.5 \mathrm{ml}$ tubes for LCM}

1) Pipette $20 \mu 1$ PicoPure Extraction Buffer into the cap of $0.5 \mathrm{ml}$ DNA Lobind tube.

Before using, mix Extraction Buffer thoroughly. Extraction Buffer may form a precipitate upon storage. If necessary, warm the XB vial to redissolve Extraction Buffer before the usage.

2) Carefully close the cap, holding the tube upside down, to let an intact droplet on the cap.

3) Freeze the tube upside down in $-20^{\circ} \mathrm{C}$

4) Prepare as many tubes as many RNA extractions you are planning

\section{LCM}

1) Place slides into the microscope's slide holder

2) Select regions on the sections for dissection by joint-cut function.

3) Cut the regions selected using 63x objective and $\sim 40 x$ laser energy

4) Place the prepared $0.5 \mathrm{ml}$ tubes in the tube holder of the microscope

5) Catapult the regions of interest into the cap using 10x objective and 50x laser energy. Target the laser beam to the joint area of the selected tissue region.

With the current protocol, we found that at least 60,000 $\mu^{2}$ tissue needs to be catapulted to reach sufficient RNA concentration ( $500 \mathrm{pg} / \mathrm{ul})$.

6) Freeze the tube upside down in liquid nitrogen

\section{D) RNA extraction (Modified protocol of the PicoPure RNA Isolation} Kit)

1) Transfer tubes from liquid nitrogen to a thermostat and incubate the tubes upside down at $42^{\circ} \mathrm{C}$ for 5 minutes

2) Snap freeze tubes in liquid nitrogen $(\sim 15 \mathrm{~s})$

3) Repeat the steps 1-2 for two more times 
4) Incubate tubes at $42^{\circ} \mathrm{C}$ for 30 minutes (tubes are still upside down)

5) Gently vortex the tubes and centrifuge for 1 minute on $8000 \mathrm{~g}$

6) Pipette $30 \mu 1$ PicoPure Extraction Buffer into the cap

7) Centrifuge tubes for 1 minute on $8000 \mathrm{~g}$

8) Incubate standing tubes at $42^{\circ} \mathrm{C}$ for 15 minutes using a heat-block.

9) During step 8 incubation, prepare Purification Columns:

a) Pipette $250 \mu$ l Conditioning Buffer (CB) onto the purification column filter membrane.

b) Incubate the RNA purification Column with Conditioning Buffer for 5 minutes at room temperature

c) Centrifuge the purification column in the provided collection tube at $16000 \times \mathrm{g}$ for one minute

10) During the last 5 minutes of step 9 incubation, prepare DNase incubation mix:

a) Thaw DNase I Stock solution.

b) Pipette $5 \mu$ DNase I Stock Solution to $35 \mu 1$ Buffer RDD. Mix gently inverting.

11) Centrifuge tubes at $1000 \mathrm{~g}$ for two minutes

12) Pipette $50 \mu \mathrm{l}$ of $70 \%$ Ethanol (EtOH) into the cell extract. Mix well by pipetting up and down. DO NOT CENTRIFUGE.

13) Pipette the cell extract and EtOH mixture into the pre-conditioned purification column.

14) To bind RNA to the column, centrifuge for 2 minutes at $100 \mathrm{x} \mathrm{g}$, immediately followed by centrifugation at $16000 \mathrm{x} \mathrm{g}$ for 30 seconds to remove flowthrough.

15) Pipette $100 \mu \mathrm{l}$ Wash Buffer (W1) into the purification column and centrifuge for one minute $8000 \mathrm{x}$.

16) Pipette $40 \mu 1$ DNase incubation mix directly into the purification column membrane. Incubate at room temperature for 15 minutes. 
17) Pipette $40 \mu$ PicoPure RNA Kit Wash Buffer 1 (W1) into the purification column membrane. Centrifuge at $8000 \mathrm{x}$ g for 15 seconds.

18) Pipette $100 \mu \mathrm{l}$ Wash Buffer 2 (W2) into the purification column and centrifuge for one minute at $8000 \mathrm{xg}$.

19) Pipette another $100 \mu \mathrm{l}$ Wash Buffer 2 (W2) into the purification column and centrifuge for two minutes at $16000 \mathrm{x} \mathrm{g}$. Re-centrifuge at $16000 \mathrm{x} \mathrm{g}$ for one minute, to ensure the elimination of any residual wash buffer from the purification column.

20) Transfer the purification column to a new $0.5 \mathrm{ml}$ microcentrifuge tube provided in the kit.

21) Pipette $10 \mu$ Elution Buffer (EB) directly onto the membrane of the purification column (Gently touch the tip of the pipette to the surface of the membrane while dispensing the elution buffer to ensure maximum absorption of EB into the membrane).

22) Incubate the purification column for one minute at room temperature

23) Centrifuge the column for one minute at $1000 \mathrm{xg}$ to distribute $\mathrm{EB}$ in the column, then for one minute at $16000 \mathrm{x} g$ to elute RNA. 


\section{Appendix 2. Cell division, hyphal growth pattern and cell wall remodeling genes among $\mathrm{H} 1$ and $\mathrm{H} 2$ upregulated genes.}

\begin{tabular}{|c|c|c|c|}
\hline \multicolumn{2}{|c|}{ Cell division and hyphal growth pattern } & \multicolumn{2}{|c|}{ Cell wall remodeling } \\
\hline Gene ID & Functional category & Gene ID & Functional category \\
\hline jgi.p|Copci_AmutBmut1|481050 & Branching & jgi.p|Copci_AmutBmut1|430008 & cellulose binding \\
\hline jgi.p|Copci_AmutBmut1|416864 & Branching & jgi.p|Copci_AmutBmut1|381999 & cellulose binding \\
\hline jgi.p|Copci_AmutBmut1|358370 & Cell cycle control & jgi.p|Copci_AmutBmut1|478785 & cellulose binding \\
\hline jgi.p|Copci_AmutBmut1|468782 & Cell cycle control & jgi.p|Copci_AmutBmut1|379418 & cellulose binding \\
\hline jgi.p|Copci_AmutBmut1|445827 & Cell cycle control & jgi.p|Copci_AmutBmut1|420416 & cellulose binding \\
\hline jgi.p|Copci_AmutBmut1|384611 & Cell cycle control & jgi.p|Copci_AmutBmut1|456674 & cellulose binding \\
\hline jgi.p|Copci_AmutBmut1|397741 & Cell cycle control & jgi.p|Copci_AmutBmut1|488885 & Chitinase \\
\hline jgi.p|Copci_AmutBmut1|364990 & Cell cycle control & jgi.p|Copci_AmutBmut1|91051 & Chitinase \\
\hline jgi.p|Copci_AmutBmut1|537439 & Cell cycle control & jgi.p|Copci_AmutBmut1|396895 & Chitinase \\
\hline jgi.p|Copci_AmutBmut1|547430 & Cell cycle control & jgi.p|Copci_AmutBmut1|486544 & Chitinase \\
\hline jgi.p|Copci_AmutBmut1|445993 & Cell cycle control & jgi.p|Copci_AmutBmut1|460405 & Chitinase \\
\hline jgi.p|Copci_AmutBmut1|438777 & Cell cycle control & jgi.p|Copci_AmutBmut1|358869 & Chitinase \\
\hline jgi.p|Copci_AmutBmut1|444706 & Cell cycle control & jgi.p|Copci_AmutBmut1|416872 & Chitinase \\
\hline jgi.p|Copci_AmutBmut1|370618 & Cell cycle control & jgi.p|Copci_AmutBmut1|90984 & Chitinase \\
\hline jgi.p|Copci_AmutBmut1|392838 & Cell cycle control & jgi.p|Copci_AmutBmut1|500546 & Chitinase \\
\hline jgi.p|Copci_AmutBmut1|393199 & Cell cycle control & jgi.p|Copci_AmutBmut1|368217 & Chitinase \\
\hline jgi.p|Copci_AmutBmut1|445594 & Cell cycle control & jgi.p|Copci_AmutBmut1|151514 & Chitinase \\
\hline jgi.p|Copci_AmutBmut1|538972 & Cell cycle control & jgi.p|Copci_AmutBmut1|380521 & Chitinase \\
\hline jgi.p|Copci_AmutBmut1|545958 & Cell cycle control & jgi.p|Copci_AmutBmut1|454814 & Chitinase \\
\hline jgi.p|Copci_AmutBmut1|459349 & Cell cycle control & jgi.p|Copci_AmutBmut1|461475 & Chitinase \\
\hline jgi.p|Copci_AmutBmut1|19106 & Cell cycle control & jgi.p|Copci_AmutBmut1|470416 & Chitinase \\
\hline jgi.p|Copci_AmutBmut1|451244 & Cell cycle control & jgi.p|Copci_AmutBmut1|445045 & Chitinase \\
\hline jgi.p|Copci_AmutBmut1|460475 & Cell cycle control & jgi.p|Copci_AmutBmut1|476132 & Chitinase \\
\hline jgi.p|Copci_AmutBmut1|478483 & Cell cycle control & jgi.p|Copci_AmutBmut1|482223 & Chitinase \\
\hline jgi.p|Copci_AmutBmut1|490912 & Cell cycle control & jgi.p|Copci_AmutBmut1|492754 & Glycoside hydrolase \\
\hline jgi.p|Copci_AmutBmut1|542482 & Cell cycle control & jgi.p|Copci_AmutBmut1|495979 & Glycoside hydrolase \\
\hline jgi.p|Copci_AmutBmut1|393128 & Cell cycle control & jgi.p|Copci_AmutBmut1|501421 & Glycoside hydrolase \\
\hline jgi.p|Copci_AmutBmut1|405337 & Cell cycle control & jgi.p|Copci_AmutBmut1|495249 & Glycoside hydrolase \\
\hline jgi.p|Copci_AmutBmut1|453453 & Cell cycle control & jgi.p|Copci_AmutBmut1|419912 & Glycoside hydrolase \\
\hline jgi.p|Copci_AmutBmut1|471958 & Cell cycle control & jgi.p|Copci_AmutBmut1|429661 & Glycoside hydrolase \\
\hline jgi.p|Copci_AmutBmut1|498411 & Cell cycle control & jgi.p|Copci_AmutBmut1|495980 & Glycoside hydrolase \\
\hline jgi.p|Copci_AmutBmut1|538026 & Cell cycle control & jgi.p|Copci_AmutBmut1|462495 & Glycoside hydrolase \\
\hline jgi.p|Copci_AmutBmut1|475755 & Cell cycle control & jgi.p|Copci_AmutBmut1|444028 & Glycoside hydrolase \\
\hline jgi.p|Copci_AmutBmut1|229833 & Cell cycle control & jgi.p|Copci_AmutBmut1|414471 & Glycoside hydrolase \\
\hline jgi.p|Copci_AmutBmut1|364989 & Cell cycle control & jgi.p|Copci_AmutBmut1|439150 & Glycoside hydrolase \\
\hline jgi.p|Copci_AmutBmut1|368398 & Cell cycle control & jgi.p|Copci_AmutBmut1|378114 & Glycoside hydrolase \\
\hline jgi.p|Copci_AmutBmut1|465387 & Cell cycle control & jgi.p|Copci_AmutBmut1|455228 & Glycoside hydrolase \\
\hline jgi.p|Copci_AmutBmut1|469725 & Cell cycle control & jgi.p|Copci_AmutBmut1|462625 & Glycoside hydrolase \\
\hline jgi.p|Copci_AmutBmut1|433472 & Cell cycle control & jgi.p|Copci_AmutBmut1|372384 & Glycoside hydrolase \\
\hline jgi.p|Copci_AmutBmut1|360681 & Cell cycle control & |jgi.p|Copci_AmutBmut1|546300 & Glycoside hydrolase \\
\hline
\end{tabular}




\begin{tabular}{|c|c|c|c|}
\hline jgi.p|Copci_AmutBmut1|444527 & Cell cycle control & jgi.p|Copci_AmutBmut1|373700 & Glycoside hydrolase \\
\hline jgi.p|Copci_AmutBmut1|51761 & Hypha_fusion & jgi.p|Copci_AmutBmut1|468708 & Glycoside hydrolase \\
\hline jgi.p|Copci_AmutBmut1|416655 & Hypha_fusion & jgi.p|Copci_AmutBmut1|471199 & Glycoside hydrolase \\
\hline jgi.p|Copci_AmutBmut1|463106 & $\begin{array}{l}\text { Hypha_fusion } \\
\text { Hyphal growth (Polarized }\end{array}$ & jgi.p|Copci_AmutBmut1|416998 & Glycoside hydrolase \\
\hline jgi.p|Copci_AmutBmut1|474296 & $\begin{array}{l}\text { growth) } \\
\text { Hyphal growth (Polarized }\end{array}$ & jgi.p|Copci_AmutBmut1|501856 & Glycoside hydrolase \\
\hline jgi.p|Copci_AmutBmut1|399236 & $\begin{array}{l}\text { growth) } \\
\text { Hyphal growth (Polarized }\end{array}$ & jgi.p|Copci_AmutBmut1|455231 & Glycoside hydrolase \\
\hline jgi.p|Copci_AmutBmut1|472903 & $\begin{array}{l}\text { growth) } \\
\text { Hyphal growth (Polarized } \\
\text { growth) }\end{array}$ & jgi.p|Copci_AmutBmut1|497633 & Glycoside hydrolase \\
\hline $\begin{array}{l}\text { jgi.p|Copci_AmutBmut1|359301 } \\
\text { jgi.p|Copci AmutBmut1|472488 }\end{array}$ & $\begin{array}{l}\text { growth) } \\
\text { Hyphal growth (Polarized } \\
\text { growth) }\end{array}$ & $\begin{array}{l}\text { jgi.p|Copci_AmutBmut1|489015 } \\
\text { jgi.p|Copci AmutBmut1|541431 }\end{array}$ & Glycoside hydrolase \\
\hline jgi.p|Copci_AmutBmut1|419142 & $\begin{array}{l}\text { Hyphal growth (Polarized } \\
\text { growth) } \\
\text { Hyphal growth (Polarized }\end{array}$ & jgi.p|Copci_AmutBmut1|517646 & Glycoside hydrolase \\
\hline jgi.p|Copci_AmutBmut1|497826 & $\begin{array}{l}\text { growth) } \\
\text { Hyphal growth (Polarized }\end{array}$ & jgi.p|Copci_AmutBmut1|441139 & Glycoside hydrolase \\
\hline jgi.p|Copci_AmutBmut1|357837 & $\begin{array}{l}\text { growth) } \\
\text { Hyphal growth (Polarized }\end{array}$ & jgi.p|Copci_AmutBmut1|445501 & Glycoside hydrolase \\
\hline jgi.p|Copci_AmutBmut1|360343 & $\begin{array}{l}\text { growth) } \\
\text { Hyphal growth (Polarized }\end{array}$ & jgi.p|Copci_AmutBmut1|357539 & Glycoside hydrolase \\
\hline jgi.p|Copci_AmutBmut1|456365 & $\begin{array}{l}\text { growth) } \\
\text { Hyphal growth (Polarized }\end{array}$ & jgi.p|Copci_AmutBmut1|227722 & Glycoside hydrolase \\
\hline jgi.p|Copci_AmutBmut1|497027 & $\begin{array}{l}\text { growth) } \\
\text { Hyphal growth (Polarized }\end{array}$ & jgi.p|Copci_AmutBmut1|145651 & Glycoside hydrolase \\
\hline jgi.p|Copci_AmutBmut1|416174 & $\begin{array}{l}\text { growth) } \\
\text { Hyphal growth (Polarized }\end{array}$ & jgi.p|Copci_AmutBmut1|378378 & Glycoside hydrolase \\
\hline jgi.p|Copci_AmutBmut1|395241 & $\begin{array}{l}\text { growth) } \\
\text { Hyphal growth (Polarized }\end{array}$ & jgi.p|Copci_AmutBmut1|411452 & Glycoside hydrolase \\
\hline jgi.p|Copci_AmutBmut1|492830 & $\begin{array}{l}\text { growth) } \\
\text { Hyphal growth (Polarized }\end{array}$ & jgi.p|Copci_AmutBmut1|422492 & Glycoside hydrolase \\
\hline jgi.p|Copci_AmutBmut1|493094 & $\begin{array}{l}\text { growth) } \\
\text { Hyphal growth (Polarized }\end{array}$ & jgi.p|Copci_AmutBmut1|462752 & Glycoside hydrolase \\
\hline jgi.p|Copci_AmutBmut1|365216 & $\begin{array}{l}\text { growth) } \\
\text { Hyphal growth (Polarized }\end{array}$ & jgi.p|Copci_AmutBmut1|437221 & Glycoside hydrolase \\
\hline jgi.p|Copci_AmutBmut1|538522 & $\begin{array}{l}\text { growth) } \\
\text { Hyphal growth (Polarized }\end{array}$ & jgi.p|Copci_AmutBmut1|449999 & Glycoside hydrolase \\
\hline jgi.p|Copci_AmutBmut1|410146 & growth) & jgi.p|Copci_AmutBmut1|444212 & Glycoside hydrolase \\
\hline jgi.p|Copci_AmutBmut1|193545 & Mitosis & jgi.p|Copci_AmutBmut1|365699 & Glycoside hydrolase \\
\hline jgi.p|Copci_AmutBmut1|469428 & Mitosis & jgi.p|Copci_AmutBmut1|371647 & Glycoside hydrolase \\
\hline jgi.p|Copci_AmutBmut1|380877 & Mitosis & jgi.p|Copci_AmutBmut1|470128 & Glycoside hydrolase \\
\hline jgi.p|Copci_AmutBmut1|406915 & Mitosis & jgi.p|Copci_AmutBmut1|486333 & Glycoside hydrolase \\
\hline jgi.p|Copci_AmutBmut1|368575 & Mitosis & jgi.p|Copci_AmutBmut1|526754 & Glycoside hydrolase \\
\hline jgi.p|Copci_AmutBmut1|464852 & Mitosis & jgi.p|Copci_AmutBmut1|546855 & Glycoside hydrolase \\
\hline jgi.p|Copci_AmutBmut1|363847 & Mitosis & jgi.p|Copci_AmutBmut1|359841 & Glycoside hydrolase \\
\hline jgi.p|Copci_AmutBmut1|363728 & Mitosis & jgi.p|Copci_AmutBmut1|425614 & Glycoside hydrolase \\
\hline jgi.p|Copci_AmutBmut1|361158 & Mitosis & jgi.p|Copci_AmutBmut1|505989 & Glycoside hydrolase \\
\hline jgi.p|Copci_AmutBmut1|464859 & Mitosis & jgi.p|Copci_AmutBmut1|91997 & Glycoside hydrolase \\
\hline jgi.p|Copci_AmutBmut1|519105 & Mitosis & jgi.p|Copci_AmutBmut1|355388 & Glycoside hydrolase \\
\hline jgi.p|Copci_AmutBmut1|464525 & Mitosis & jgi.p|Copci_AmutBmut1|250720 & Glycoside hydrolase \\
\hline jgi.p|Copci_AmutBmut1|160113 & Mitosis & jgi.p|Copci_AmutBmut1|474165 & Glycoside hydrolase \\
\hline jgi.p|Copci_AmutBmut1|502170 & Mitosis & jgi.p|Copci_AmutBmut1|320571 & Glycoside hydrolase \\
\hline jgi.p|Copci_AmutBmut1|439045 & Mitosis & jgi.p|Copci_AmutBmut1|462081 & Glycoside hydrolase \\
\hline jgi.p|Copci_AmutBmut1|261810 & Mitosis & jgi.p|Copci_AmutBmut1|484892 & Glycoside hydrolase \\
\hline jgi.p|Copci_AmutBmut1|60974 & Mitosis & jgi.p|Copci_AmutBmut1|384328 & Glycoside hydrolase \\
\hline jgi.p|Copci_AmutBmut1|191447 & Mitosis & jgi.p|Copci_AmutBmut1|540056 & Glycoside hydrolase \\
\hline jgi.p|Copci_AmutBmut1|379984 & Mitosis & jgi.p|Copci_AmutBmut1|179843 & Glycoside hydrolase \\
\hline
\end{tabular}




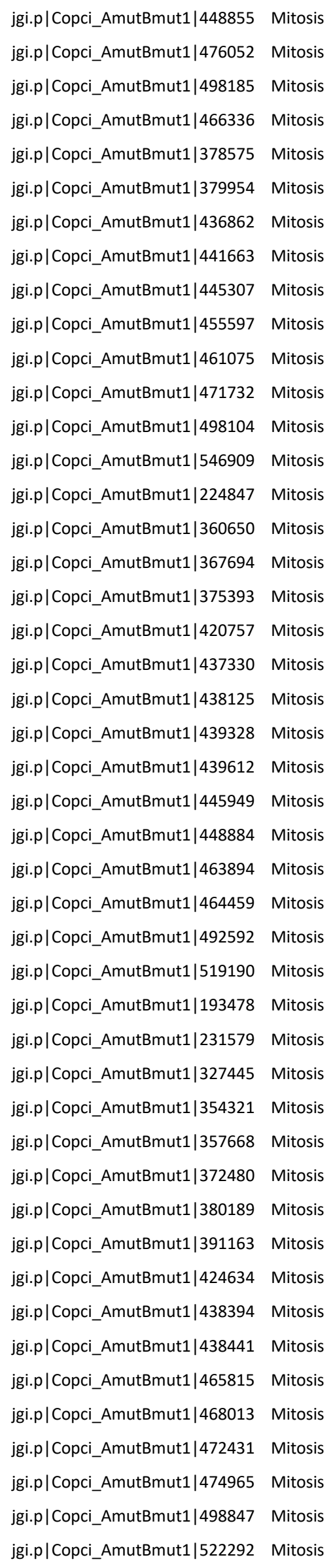

jgi.p|Copci_AmutBmut1|356620 jgi.p|Copci_AmutBmut1|376687 jgi.p|Copci_AmutBmut1|380156 jgi.p|Copci_AmutBmut1|473021 jgi.p|Copci_AmutBmut1|268213 jgi.p|Copci_AmutBmut1|436813 jgi.p|Copci_AmutBmut1|451158 jgi.p|Copci_AmutBmut1|466967 jgi.p|Copci_AmutBmut1|468626 jgi.p|Copci_AmutBmut1|485043 jgi.p|Copci_AmutBmut1|490988 jgi.p|Copci_AmutBmut1|496928 jgi.p|Copci_AmutBmut1|500464 jgi.p|Copci_AmutBmut1|525904 jgi.p|Copci_AmutBmut1|542232 jgi.p|Copci_AmutBmut1|504495 jgi.p|Copci_AmutBmut1|441604 jgi.p|Copci_AmutBmut1|540817 jgi.p|Copci_AmutBmut1|436904 jgi.p|Copci_AmutBmut1|443934 jgi.p|Copci_AmutBmut1|448216 jgi.p|Copci_AmutBmut1|467674 jgi.p|Copci_AmutBmut1|494762
Glycoside hydrolase

Glycoside hydrolase Glycoside hydrolase Glycoside hydrolase Glycoside hydrolase Glycoside hydrolase Glycoside hydrolase Glycoside hydrolase Glycoside hydrolase Glycoside hydrolase Glycoside hydrolase Glycoside hydrolase Glycoside hydrolase Glycoside hydrolase Glycoside hydrolase Glycosyl transferase Glycosyl transferase Glycosyl transferase Glycosyl transferase Glycosyl transferase Glycosyl transferase Glycosyl transferase Polysaccharide lyase 


\begin{tabular}{|c|c|}
\hline jgi.p|Copci_AmutBmut1|5312 & Mitosis \\
\hline jgi.p|Copci_AmutBmut1|442128 & Mitosis \\
\hline jgi.p|Copci_AmutBmut1|443347 & Mitosis \\
\hline jgi.p|Copci_AmutBmut1|244667 & Mitosis \\
\hline jgi.p|Copci_AmutBmut1|365564 & Mitosis \\
\hline jgi.p|Copci_AmutBmut1|539558 & Mitosis \\
\hline jgi.p|Copci_AmutBmut1|358605 & Mitosis \\
\hline jgi.p|Copci_AmutBmut1|373301 & Mitosis \\
\hline jgi.p|Copci_AmutBmut1|377255 & Mitosis \\
\hline jgi.p|Copci_AmutBmut1|411203 & Mitosis \\
\hline jgi.p|Copci_AmutBmut1|446459 & Mitosis \\
\hline jgi.p|Copci_AmutBmut1|492299 & Mitosis \\
\hline jgi.p|Copci_AmutBmut1|267343 & Mitosis \\
\hline jgi.p|Copci_AmutBmut1|378537 & Mitosis \\
\hline jgi.p|Copci_AmutBmut1|441190 & Mitosis \\
\hline jgi.p|Copci_AmutBmut1|466100 & Mitosis \\
\hline jgi.p|Copci_AmutBmut1|521062 & Mitosis \\
\hline jgi.p|Copci_AmutBmut1|358096 & Mitosis \\
\hline jgi.p|Copci_AmutBmut1|511209 & Mitosis \\
\hline jgi.p|Copci_AmutBmut1|173502 & Mitosis \\
\hline jgi.p|Copci_AmutBmut1|360237 & Mitosis \\
\hline jgi.p|Copci_AmutBmut1|371359 & Mitosis \\
\hline jgi.p|Copci_AmutBmut1|498107 & Mitosis \\
\hline jgi.p|Copci_AmutBmut1|500550 & Mitosis \\
\hline jgi.p|Copci_AmutBmut1|501207 & Mitosis \\
\hline jgi.p|Copci_AmutBmut1|543592 & Septatior \\
\hline jgi.p|Copci_AmutBmut1|479908 & Septati \\
\hline jgi.p|Copci_AmutBmut1|508178 & Septati \\
\hline jgi.p|Copci_AmutBmut1|492754 & Septati \\
\hline jgi.p|Copci_AmutBmut1|367587 & Septati \\
\hline jgi.p|Copci_AmutBmut1|455217 & Septati \\
\hline jgi.p|Copci_AmutBmut1|415573 & Septa \\
\hline jgi.p|Copci_AmutBmut1|466786 & Septat \\
\hline 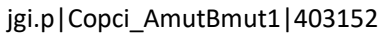 & \\
\hline
\end{tabular}




\section{Appendix 3. H1 and $\mathrm{H} 2$ upregulated genes related to beta-glucan metabolism.}

\begin{tabular}{|c|c|c|c|}
\hline Protein ID & Upregulated & Annotations & $\begin{array}{l}\text { Metabolic } \\
\text { pathway }\end{array}$ \\
\hline Copci_AmutBmut1_414471 & H1 & $\begin{array}{l}\text { S. cerevisiae ortholog: Major exo- } \\
\text { 1,3-beta-glucanase of the cell } \\
\text { wall; involved in cell wall beta- } \\
\text { glucan assembly. IPR: GH5. Kiss } \\
\text { et al. 2019: Cell wall }\end{array}$ & Anabolism in cell wall \\
\hline Copci_AmutBmut1_357539 & $\mathrm{H} 1$ & $\begin{array}{l}\text { IPR: Beta-glucan synthesis- } \\
\text { associated, GH16; Kiss et al. } \\
\text { 2019: Cell wall }\end{array}$ & Anabolism in cell wall \\
\hline Copci_AmutBmut1_373700 & $\mathrm{H} 1, \mathrm{H} 2$ nodulus & $\begin{array}{l}\text { GO: glucan 1,3-beta-glucosidase } \\
\text { activity; IPR: GH5; Kiss et al. } \\
\text { 2019: Cell wall }\end{array}$ & Catabolism in cell wall \\
\hline Copci_AmutBmut1_546300 & $\mathrm{H} 1$ & $\begin{array}{l}\text { S. pombe ortholog: cell wall } \\
\text { glucan } \quad \text { 1,3-beta-glucosidase } \\
\text { Exg3; IPR: GH5; Kiss et al. 2019: } \\
\text { Cell wall }\end{array}$ & Catabolism in cell wall \\
\hline Copci_AmutBmut1_541431 & $\mathrm{H} 1$ & $\begin{array}{l}\text { GO: beta-glucosidase activity; } \\
\text { IPR: GH3 }\end{array}$ & Catabolism \\
\hline Copci_AmutBmut1_439150 & $\begin{array}{l}\text { H1, H2 nodulus, } \\
\text { H2 UV }\end{array}$ & $\begin{array}{l}\text { IPR: Beta-glucan synthesis- } \\
\text { associated, GH16; Kiss et al. } \\
\text { 2019: Cell wall }\end{array}$ & Anabolism in cell wall \\
\hline Copci_AmutBmut1_91997 & H2 nodulus & $\begin{array}{l}\text { IPR: Beta-glucan synthesis- } \\
\text { associated, GH16; Kiss et al. } \\
\text { 2019: Cell wall }\end{array}$ & Anabolism in cell wall \\
\hline Copci_AmutBmut1_484892 & H2 nodulus & $\begin{array}{l}\text { GO\&KEGG: glucan 1,3-beta- } \\
\text { glucosidase activity; IPR: GH5; } \\
\text { Kiss et al. 2019: Cell wall }\end{array}$ & Catabolism in cell wall \\
\hline Copci_AmutBmut1_444212 & H2 UV & $\begin{array}{l}\text { A. nidulans ortholog: beta- } \\
\text { glucosidase with a predicted role } \\
\text { in degradation of glucans; GO: } \\
\text { beta-glucosidase activity; IPR: } \\
\text { GH3 }\end{array}$ & Catabolism \\
\hline Copci_AmutBmut1_470128 & H2 UV & $\begin{array}{l}\text { GO\&KEGG: glucan 1,3-beta- } \\
\text { glucosidase activity; IPR: GH5; } \\
\text { Kiss et al. 2019: Cell wall }\end{array}$ & Catabolism in cell wall \\
\hline Copci_AmutBmut1_425614 & H2 UV & $\begin{array}{l}\text { GO\&KEGG: glucan 1,3-beta- } \\
\text { glucosidase activity; IPR: GH3 }\end{array}$ & Catabolism \\
\hline
\end{tabular}




\begin{tabular}{|l|l|l|l|}
\hline Copci_AmutBmut1_505989 & H2 UV & $\begin{array}{l}\text { A. nidulans ortholog: beta-(1,6)- } \\
\text { glucanase activity, involved in } \\
\text { degradation of glucans. } \\
\text { GO\&KEGG: glucan 1,3-beta- } \\
\text { glucosidase activity; IPR: GH5; }\end{array}$ & \\
\hline Copci_AmutBmut1_439150 & H2 Nodulus + & $\begin{array}{l}\text { IPR: Beta-glucan synthesis- } \\
\text { associated, GH16, Kiss et al. } \\
\text { 2019: Cell wall }\end{array}$ & Anabolism in cell wall \\
\hline Copci_AmutBmut1_496928 & H2 Nodulus + & $\begin{array}{l}\text { IPR: Beta-glucan synthesis- } \\
\text { associated, GH16, Kiss et al. } \\
\text { 2019: Cell wall }\end{array}$ & Anabolism in cell wall \\
\hline Copci_AmutBmut1_376687 & H2 Nodulus + & $\begin{array}{l}\text { A. nidulans ortholog: Putative } \\
\text { beta-glucosidase with a predicted } \\
\text { role in polysaccharide } \\
\text { degradation; IPR\&GO: cellulose } \\
\text { binding;beta-glucosidase activity, } \\
\text { GH3; KEGG: Beta-glucosidase }\end{array}$ & Catabolism \\
\hline Copci_AmutBmut1_542232 & H2 Nodulus + & $\begin{array}{l}\text { A. nidulans ortholog: Beta- } \\
\text { glycosidase transcriptionally } \\
\text { induced by growth on xylose; } \\
\text { IPR, GO\&KEGG: beta- } \\
\text { glucosidase activity, GH1 }\end{array}$ & \\
\hline
\end{tabular}

